\title{
6 CARACTERIZACIÓN DE LOS DIFERENTES ACTORES DEL SISTEMA SANITARIO PÚBLICO CATALÁN
}

Como ha quedado dicho, el trabajo empírico de contraste de las hipótesis formuladas a partir del desarrollo teórico realizado en la primera parte del estudio, se ha aplicado al caso del Sistema de Innovación que constituye el Sector Sanitario Público Catalán.

El objetivo del presente capítulo es realizar la caracterización ${ }^{1}$ de los diferentes actores que han sido objeto del estudio empírico. El SSPC y dentro del los actores creados por la LOSC, constituye el núcleo de nuestro estudio y, por tanto, es éste el ámbito de nuestro análisis.

Esta caracterización del objeto de estudio incluye tanto descripciones a partir de diferentes estudios sobre el sector como de otras que son secundarias dentro de los objetivos del presente trabajo pero necesarias para hacer una descripción apropiada de la realidad a estudiar.

Ley general de sanidad atribuye tanto al Estado como a las comunidades autónomas y a las otras administraciones públicas la organización y el desarrollo de las acciones sanitarias según una concepción integral de la salud, siguiendo la definición de la Organización Mundial de la Salud de concebirla no sólo como una ausencia de enfermedad sino como un estado completo de bienestar físico, mental y social.

En el ámbito sanitario la Generalitat de Catalunya tiene competencias en sanidad interior y seguridad social, de manera que puede organizar y administrar todos los servicios relacionados con estas materias y ejercer la tutela de las instituciones, entidades y fundaciones sociosanitarias. También tiene como competencia propia la planificación sanitaria.

El Departament de Sanitat i Seguretat Social es el órgano que ejerce las funciones y los servicios en materia de sanidad que son competencia de la

1 Recordando la definición de la Real Academia Española-Diccionario de la Lengua Española, 21 Edición. Tomo I. 1997, p. 406.

- Caracterización: acción y efecto de caracterizar o caracterizarse.

- Caracterizar: determinar los atributos peculiares de una persona o cosa, de modo que claramente se distinga de las demás. 
Generalitat. El Departamento dispone de una estructura articulada a través de las direcciones generales de Recursos Sanitaris y de Salut Pública, con una presencia territorial a través de delegaciones en Barcelona, Tarragona, Lleida y Girona. Además de estos órganos, cuenta con unos organismos adscritos:

- Servei Català de la Salut.

- Institut Català de la Salut.

- Institut d'Estudis de la Salut.

\subsection{SERVEI CATALÀ DE LA SALUT}

\subsubsection{MISIÓN Y OBJETIVOS ESTRATÉGICOS}

El Servei Català de la Salut _ SCS - es un ente que debe garantizar la prestación de los servicios sanitarios de cobertura pública de Catalunya y, por tanto, es el responsable de velar por la equidad, la calidad y la eficiencia de los servicios que recibe la población.

Para cumplir con esta misión, el Servei Català de la Salut pone a disposición de la red sanitaria de utilización pública de Catalunya su organización corporativa - servicios matrices, regiones y sectores sanitarios - y sus recursos humanos.

También fomenta fórmulas como los consorcios, las empresas públicas y las cesiones de gestión para avanzar en una administración más eficiente del sistema sanitario.

El compromiso con los ciudadanos requiere la existencia de unos objetivos asumidos por el conjunto del Servei Català de la Salut ${ }^{2}$ :

- Planificar las prioridades de la atención sanitaria a partir de las necesidades de salud de la población y no solo a partir de la oferta.

- Conseguir el equilibrio económico del sector de la sanidad, en una situación compleja de incremento de la demanda de servicios y de contención del gasto del sector público.

- Gestionar las prestaciones de manera eficiente para optimizar la relación entre los recursos destinados y la calidad obtenida, sin perder de vista la equidad en el acceso a los servicios sanitarios como

2 Siguiendo la Memòria d'ativitat 1996 del Servei Català de la Salut [1997, p. 17]. 
principio básico del sistema.

- Promover la participación de los profesionales en la consolidación del sistema sanitario público catalán y en la consecución de los objetivos del Servei Català de la Salut.

- Mejorar la calidad de servicio al ciudadano, tanto en el trato como en la asistencia.

- Dotarse de instrumentos organizativos que potencien la calidad, la profesionalidad y la responsabilidad en el trabajo.

FIGURA 6.1. Planificación del desarrollo de la asistencia sanitaria pública.

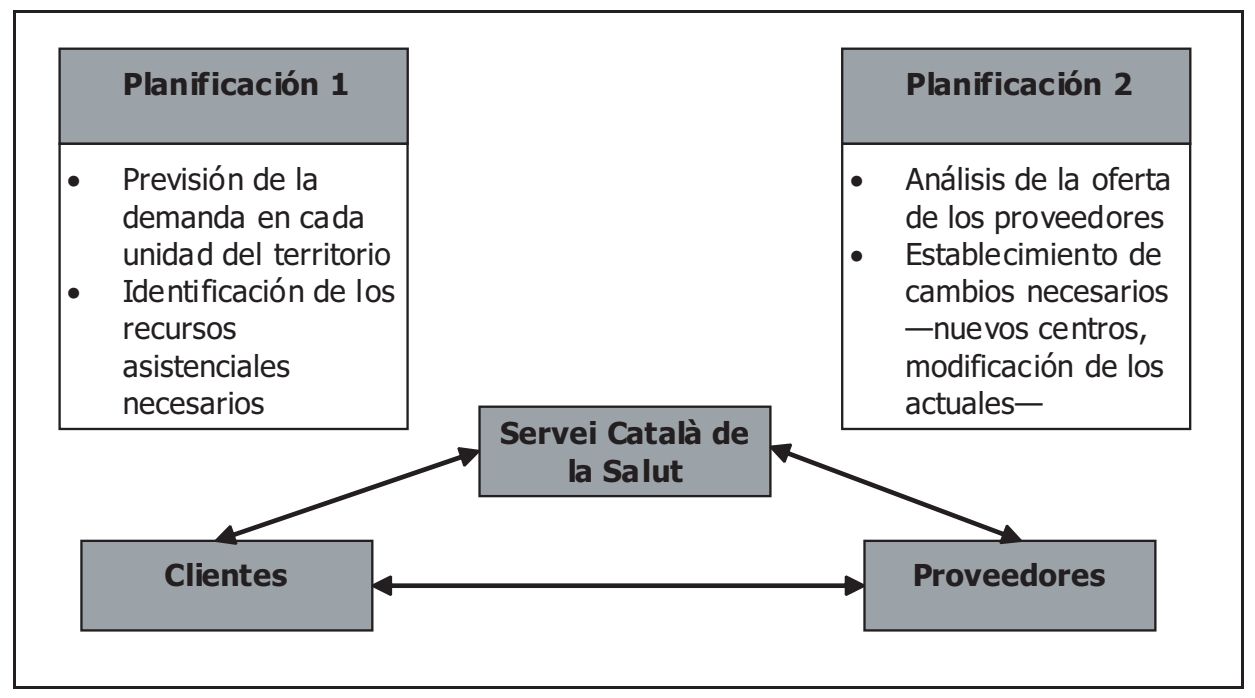

(C) McKinsey \& Company [1992, p. 8].

\subsubsection{LA XARXA SANITÀRIA D'UTILITZACIÓ PÚBLICA DE CATALUNYA}

La red sanitaria de utilización pública de Catalunya - Xarxa Sanitària d'Utilització Pública de Catalunya XSUPC - está configurada por todos los centros, servicios y profesionales que garantizan la asistencia a la población de Catalunya y cuja financiación es pública.

El Servei Català de la Salut planifica y financia todos estos recursos sanitarios, económicos y humanos y los pone al servicio de los ciudadanos con unos criterios de equidad, calidad y eficiencia. 
FIGURA 6.2. Mercado de asistencia sanitaria pública.

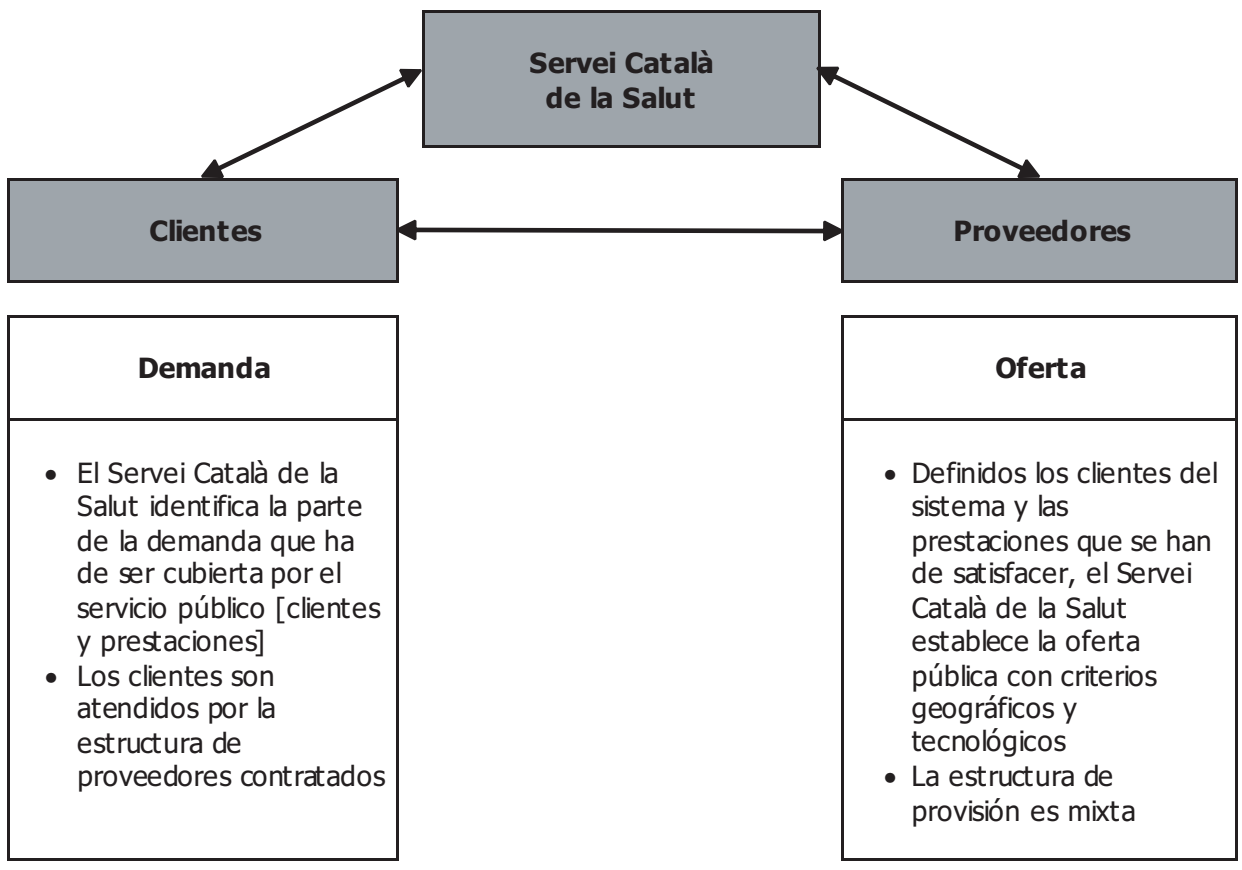

(C) McKinsey \& Company [1992, p. 7].

\subsubsection{RECURSOS SANITARIOS}

Los recursos sanitarios que el Servei Català de la Salut pone al alcance de los ciudadanos constituyen la red sanitaria de utilización pública de Catalunya. El Servei Català de la Salut planifica estos recursos con criterios de accesibilidad y de equilibrio territorial.

Cuando una región sanitaria no dispone de un recurso concreto, sus pacientes son atendidos en servicios alternativos de la misma región o bien son derivados a otras regiones sanitarias, como ocurre, por ejemplo, con los hospitales de alta tecnología y en estos momentos con los hospitales monográficos de internamiento para pacientes crónicos en salud mental. 
FIGURA 6.3. Competencia en el marco de la asistencia sanitaria pública.

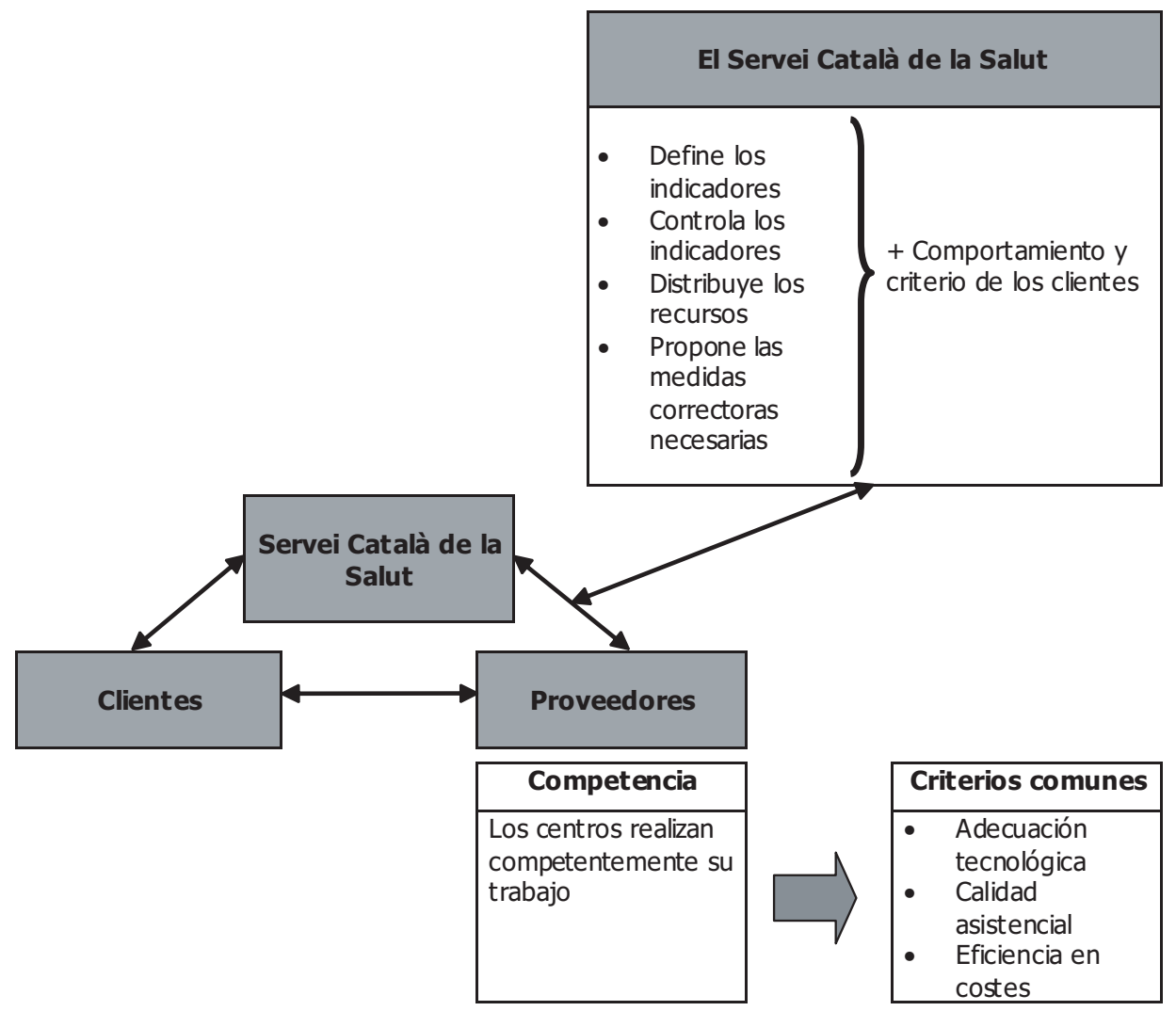

(C) Mckinsey \& Company [1992, p. 9].

\subsubsection{ATENCIÓN PRIMARIA}

La atención primaria de salud es uno de los niveles fundamentales de la asistencia sanitaria. El área básica de salud — ABS — se configura como la unidad territorial para el desarrollo de la atención primaria y la unidad elemental de la organización territorial del Servei Català de la Salut. La estructura organizativa para configurar el núcleo de servicios básicos de atención primaria es el equipo de atención primaria — EAP_, compuesto por un conjunto de profesionales sanitarios y no sanitarios que desarrollan y dirigen sus actividades de salud al conjunto de la población que comprende el ABS. Cada ABS tiene un centro de referencia - el centro de atención primaria CAP-.

La reforma de la atención primaria — $\mathrm{RAP}$ - sigue un proceso gradual de implantación de acuerdo con las disponibilidades presupuestarias y que se irá 
completando hasta llegar a la cobertura de la totalidad de la población. La gradualidad de la extensión del nuevo modelo hace que actualmente convivan en Catalunya dos redes de atención primaria, las llamadas red reformada y red no reformada.

TABLA 6.1. Recursos de atención primaria de la red sanitaria de utilización pública de Catalunya, 1996.

\begin{tabular}{|l|r|r|r|r|}
\hline $\begin{array}{l}\text { Región } \\
\text { sanitaria }\end{array}$ & $\begin{array}{r}\text { ABS en } \\
\text { funcionamiento }\end{array}$ & $\begin{array}{r}\text { ABS } \\
\text { Previstas }^{\mathbf{1}}\end{array}$ & $\begin{array}{r}\text { \% ABS en } \\
\text { funcionamiento }\end{array}$ & $\begin{array}{r}\text { \% de población } \\
\text { cubierta }\end{array}$ \\
\hline Lleida & 22 & 29 & 75,9 & 73,7 \\
\hline Tarragona & 22 & 33 & 66,7 & 62,7 \\
\hline Tortosa & 7 & 9 & 77,8 & 69,0 \\
\hline Girona & 20 & 36 & 55,6 & 50,5 \\
\hline Costa de Ponent & 41 & 57 & 71,9 & 73,3 \\
\hline $\begin{array}{l}\text { Barcelonès Nord } \\
\text { i Maresme }\end{array}$ & 18 & 41 & 43,9 & 49,5 \\
\hline Centre & 51 & 71 & 71,8 & 70,1 \\
\hline Barcelona Ciutat & 27 & 66 & 40,9 & 35,4 \\
\hline Total & $\mathbf{2 0 8}$ & $\mathbf{3 4 2}$ & $\mathbf{6 0 , 8}$ & $\mathbf{5 7 , 0}$ \\
\hline 1. El 17 d & & & & \\
\hline
\end{tabular}

1. El 17 de enero de 1996 fue publicado en el DOGC la orden de 28 de diciembre, por la cual se modifica la delimitación de diversas áreas básicas de salud y aumenta en 4 su número total.

(C) Memòria d'activitat 1996 del Servei Català de la Salut [1997, p. 19].

\subsubsection{ATENCIÓN HOSPITALARIA3}

La atención especializada en Catalunya se presta mayoritariamente en los centros hospitalarios que configuran la Red Hospitalaria de Utilización Pública —XHUP—.

3 Los hospitales en Catalunya se clasifican en 4 grupos, siguiendo parcialmente al Decreto 179/1997, de 22 de julio, DOGC núm. 2445 de 31.07.1997, p. 8720:

- Grupo 1: Hospital General Básico y Hospital Aislado.

- Grupo 2: Hospital General Básico: Es aquel que da respuesta completa a los requerimientos habituales de la población.

- Grupo 3: Hospital de Referencia: Destinado a resolver la práctica totalidad de los problemas de salud susceptibles de curación y mejoría, excepto aquellos que requiere recursos tecnológicos altamente especializados.

- Grupo 4: Hospital de alta tecnología: Asume las patologías de baja incidencia no asumibles por los hospitales mencionados anteriormente y dispone de las llamadas supraespecialidades y de las nuevas tecnologías diagnóstico terapéuticas. 
TABLA 6.2. Recursos de atención hospitalaria de la XHUP, 1996.

\begin{tabular}{|c|c|c|c|c|c|c|c|c|}
\hline \multirow[t]{2}{*}{$\begin{array}{l}\text { Región } \\
\text { sanitaria }\end{array}$} & \multicolumn{2}{|c|}{$\begin{array}{l}\text { Hospitales } \\
\text { Grupo } 1-2\end{array}$} & \multicolumn{2}{|c|}{$\begin{array}{c}\text { Hospitales } \\
\text { Grupo } 3\end{array}$} & \multicolumn{2}{|c|}{$\begin{array}{l}\text { Hospitales } \\
\text { Grupo } 4\end{array}$} & \multicolumn{2}{|c|}{ Total } \\
\hline & Centros & Camas & Centros & Camas & Centros & Camas & Centros & Camas \\
\hline Lleida & 6 & 450 & 1 & 435 & 0 & 0 & 7 & 885 \\
\hline Tarragona & 4 & 415 & 2 & 622 & 0 & 0 & 6 & 1.037 \\
\hline Tortosa & 0 & 0 & 1 & 205 & 0 & 0 & 1 & 205 \\
\hline Girona & 7 & 668 & 1 & 436 & 0 & 0 & 8 & 1.104 \\
\hline Costa de Ponent & 5 & 483 & 3 & 633 & 3 & 1.340 & 11 & 2.486 \\
\hline $\begin{array}{l}\text { Barcelonès Nord i } \\
\text { Maresme }\end{array}$ & 3 & 491 & 1 & 276 & 1 & 573 & 5 & 1.340 \\
\hline Centre & 7 & 558 & 7 & 2.557 & 0 & 0 & 14 & 3.115 \\
\hline Barcelona Ciutat & 5 & 541 & 5 & 1.708 & 6 & 3.203 & 16 & 5.452 \\
\hline Total & 37 & 3.606 & 21 & 6.872 & 10 & 5.116 & 68 & 15.624 \\
\hline
\end{tabular}

(C) Memòria d'activitat 1996 del Servei Català de la Salut [1997, p. 20] y elaboración propia.

\subsubsection{ATENCIÓN EXTRAHOSPITALARIA}

La atención extrahospitalaria cubre un conjunto de prestaciones asistenciales que complementan la atención primaria de salud y la atención especializada. Los proveedores de servicios extrahospitalarios contratados por el Servei Català de la Salut atienden las demandas generadas por todas las regiones sanitarias.

TABLA 6.3. Proveedores de servicios extrahospitalarios de la red sanitaria de utilización pública de Catalunya, 1996.

\begin{tabular}{lr} 
Diagnóstico por la imagen & 26 \\
Laboratorios de análisis clínicos & 10 \\
Laboratorios de medicina nuclear & 5 \\
Centros de rehabilitación & 25 \\
Transporte sanitario & 48 \\
Consultorios de especialidades & 16 \\
Entidades diversas & 14 \\
Oxigenoterapia y tratamientos respiratorios domiciliares & 7 \\
Centros de planificación familiar & 61 \\
Centros polivalentes & 23 \\
Centros de litotricia & 5 \\
Centros de diálisis & 42 \\
Neurología & 4 \\
\hline
\end{tabular}

(C) Memòria d'activitat 1996 del Servei Català de la Salut [1997, p. 21]. 


\subsubsection{ATENCIÓN SOCIOSANITARIA}

Para poder cubrir las necesidades de los pacientes de edad avanzada o de los afectados por enfermedades crónicas que presentan como denominador común un grado variable de incapacidad funcional resultante, el Servei Català de la Salut contrata, a través del Programa Vida a los Años, un amplio abanico de recursos en todos los niveles asistenciales. De entre este amplio abanico hay que distinguir los recursos de internamiento ubicados en centros sociosanitarios o en hospitales, los recursos comunitarios de apoyo domiciliario a los equipos de atención primaria -PADES—, y los equipos de apoyo y evaluación hospitalarios -UFISS-.

TABLA 6.4. Recursos sociosanitarios de la red sanitaria de utilización pública de Catalunya, 1996.

\begin{tabular}{|c|c|c|c|c|c|c|c|}
\hline $\begin{array}{l}\text { Región } \\
\text { sanitaria }\end{array}$ & $\begin{array}{r}\text { Larga } \\
\text { estancia }\end{array}$ & $\begin{array}{r}\text { Convale } \\
\text { cencia }\end{array}$ & $\begin{array}{r}\text { Curas } \\
\text { paliativas }\end{array}$ & SIDA & Total & Centros & $\begin{array}{c}\text { Camas } \\
\text { Sociales }^{1}\end{array}$ \\
\hline Lleida & 245 & 37 & 20 & 0 & 302 & 6 & 33 \\
\hline Tarragona & 222 & 79 & 15 & 3 & 319 & 8 & 74 \\
\hline Tortosa & 203 & 40 & 15 & 0 & 258 & 2 & 58 \\
\hline Girona & 341 & 51 & 10 & 0 & 402 & 10 & 60 \\
\hline Costa de Ponent & 952 & 114 & 20 & 0 & 1.086 & 14 & 15 \\
\hline $\begin{array}{l}\text { Barcelonès Nord i } \\
\text { Maresme }\end{array}$ & 492 & 70 & 12 & 0 & 574 & 8 & 105 \\
\hline Centre & 757 & 177 & 65 & 18 & 1.017 & 16 & 74 \\
\hline Barcelona Ciutat & 947 & 288 & 93 & 49 & 1.377 & 17 & 88 \\
\hline Total & 4.159 & 856 & 250 & 70 & 5.335 & 81 & 507 \\
\hline
\end{tabular}

(C) Memòria d'activitat 1996 del Servei Català de la Salut [1997, p. 22].

Como recursos de internamiento actualmente se disponen de:

- Unidades de larga estancia.

- Unidades de convalecencia

- Unidades de curas paliativas.

- Unidades de SIDA.

Existen también, ubicados en centros sociosanitarios, recursos sin pernocta, que son los hospitales de día sociosanitarios. 
TABLA 6.5. Recursos sociosanitarios de la red sanitaria de utilización pública de Catalunya, 1996.

\begin{tabular}{|l|r|r|r|r|}
\hline \multicolumn{5}{|c|}{ Hospital de día } \\
\hline Región sanitaria & Número & Plazas & PADES & UFISS \\
\hline Lleida & 2 & 30 & 3 & 3 \\
Tarragona & 4 & 60 & 4 & 4 \\
Tortosa & 1 & 20 & 1 & 1 \\
Girona & 4 & 52 & 2 & 3 \\
Costa de Ponent & 5 & 85 & 8 & 10 \\
Barcelonès Nord i Maresme & 6 & 126 & 5 & 7 \\
Centre & 9 & 191 & 8 & 7 \\
Barcelona Ciutat & 7 & 130 & 13 & 5 \\
\hline Total & $\mathbf{3 8}$ & $\mathbf{6 9 4}$ & $\mathbf{4 4}$ & $\mathbf{4 0}$ \\
\hline
\end{tabular}

(C) Memòria d'activitat 1996 del Servei Català de la Salut [1997, p. 22].

\subsubsection{BANCOS DE SANGRE HOSPITALARIOS}

El banco de sangre es la estructura hemoterápica responsable de la donación y la transfusión en el centro hospitalario.

TABLA 6.6. Bancos de sangre de la RSUP de Catalunya, 1996.

\begin{tabular}{|l|l|}
\hline Leida & - Hospital de Lleida Arnau de Vilanova \\
\hline Tarragona & - Hospital de Tarragona Joan XXIII \\
& - Hospital de Sant Pau i Santa Tecla -Tarragona- \\
\hline Tortosa & - Hospital Verge de la Cinta -Tortosa- \\
\hline Girona & - Hospital de Girona Doctor Josep Trueta \\
\hline Costa de Ponent & - Hospital Prínceps d'Espanya -l'Hospitalet de Llobregat- \\
& - Hospital Sant Joan de Déu -Esplugues de Llobregat- \\
\hline Barcelonès Nord i Maresme & - Hospital Residència Sant Camil -Sant Pere de Ribes- \\
\hline Centre & - Banc de Sang de la Creu Roja de Manresa \\
& - Hospital Mútua de Terrassa \\
& - Hospital General de Granollers \\
\hline Barcelona Ciutat & - Serveis Sanitaris de Referència-Centre de Transfusió i Banc de \\
& - Teixits - Hospital de la Santa Creu i Sant Pau \\
& - Hospital Clínic i Provincial de Barcelona \\
& - Hospital de Barcelona \\
& - Banc de Sang de la Creu Roja de Barcelona \\
& - Clínica Sagrada Ramília \\
& - Clínica Corach \\
\hline
\end{tabular}

(C) Memòria d'activitat 1996 del Servei Català de la Salut [1997, p. 25]. 


\subsubsection{ATENCIÓN PSIQUIÁTRICA Y SALUD MENTAL}

Los recursos sanitarios de atención psiquiátrica y salud mental comprenden las vertientes asistenciales de la atención ambulatoria en los centros de salud mental —atención especializada de apoyo a la atención primaria—, de la atención hospitalaria — total y parcial — en hospitales generales y en hospitales psiquiátricos y de rehabilitación en los centros de día.

TABLA 6.7. Centros de salud mental de la red sanitaria de utilización pública de Catalunya, 1996.

\begin{tabular}{|l|r|r|r|r|}
\hline \multirow{2}{*}{ Región sanitaria } & \multicolumn{2}{|c|}{ Infantil juvenil } & \multicolumn{2}{|c|}{ Adultos } \\
\cline { 2 - 4 } & Centros & $\begin{array}{r}\text { Consultas } \\
\text { periféricas }\end{array}$ & Centros & $\begin{array}{r}\text { Consultas } \\
\text { periféricas }\end{array}$ \\
\hline Lleida & 1 & 0 & 6 & 2 \\
Tarragona & 1 & 3 & 6 & 0 \\
Tortosa & 0 & 2 & 2 & 0 \\
Girona & 2 & 2 & 7 & 0 \\
Costa de Ponent & 6 & 3 & 11 & 0 \\
Barcelonès Nord i Maresme & 4 & 0 & 7 & 0 \\
Centre & 7 & 3 & 12 & 3 \\
Barcelona Ciutat & 10 & 1 & 15 & 1 \\
\hline Total & $\mathbf{3 1}$ & $\mathbf{1 4}$ & $\mathbf{6 6}$ & $\mathbf{6}$ \\
\hline
\end{tabular}

(C) Memòria d'activitat 1996 del Servei Català de la Salut [1997, p. 23].

TABLA 6.8. Camas de psiquiatría de la red sanitaria de utilización pública de Catalunya, 1996.

\begin{tabular}{|l|r|r|r|r|r|}
\hline Región sanitaria & Agudos & Subagudos & Crónicos & $\begin{array}{r}\text { Hospitales } \\
\text { de la XHUP }\end{array}$ & Total \\
\hline Lleida & 0 & 0 & 52 & 14 & 66 \\
Tarragona & 51 & 35 & 1.118 & 0 & 1.204 \\
Tortosa & 0 & 0 & 0 & 0 & 0 \\
Girona & 40 & 16 & 440 & 0 & 496 \\
Costa de Ponent & 236 & 167 & 1.553 & 58 & 2.014 \\
Barcelonès Nord i Maresme & 96 & 44 & 412 & 0 & 552 \\
Centre & 0 & 0 & 0 & 83 & 83 \\
Barcelona Ciutat & 126 & 52 & 81 & 80 & 339 \\
\hline Total & $\mathbf{5 4 9}$ & $\mathbf{3 1 4}$ & $\mathbf{3 . 6 5 6}$ & $\mathbf{2 3 5}$ & $\mathbf{4 . 7 5 4}$ \\
\hline
\end{tabular}

(C) Memòria d'activitat 1996 del Servei Català de la Salut [1997, p. 23]. 
TABLA 6.9. Hospitales de día de la red sanitaria de utilización pública de Catalunya, 1996.

\begin{tabular}{|l|r|r|}
\hline Región sanitaria & Hospitales de día & Plazas \\
\hline Costa de Ponent & 2 & 65 \\
Barcelonès Nord i Maresme & 1 & 21 \\
Centre & 1 & 10 \\
Barcelona Ciutat & 2 & 90 \\
\hline Total & $\mathbf{6}$ & $\mathbf{1 8 6}$ \\
\hline
\end{tabular}

(C) Memòria d'activitat 1996 del Servei Català de la Salut [1997, p. 24].

TABLA 6.10. Recursos de rehabilitación en salud mental de la red de utilización pública de Catalunya, 1996.

\begin{tabular}{|l|r|r|r|r|}
\hline \multirow{2}{*}{ Región sanitaria } & \multicolumn{2}{|c|}{ Infantil y juvenil } & \multicolumn{2}{|c|}{ Adultos } \\
\cline { 2 - 5 } & Centros de día & Plazas & Centros de día & Plazas \\
\hline Lleida & 1 & 18 & 1 & 20 \\
Tarragona & 0 & 0 & 2 & 35 \\
Tortosa & 0 & 0 & 0 & 0 \\
Girona & 0 & 0 & 5 & 100 \\
Costa de Ponent & 1 & 39 & 8 & 143 \\
Barcelonès Nord i Maresme & 1 & 9 & 2 & 60 \\
Centre & 2 & 28 & 8 & 163 \\
Barcelona Ciutat & 3 & 76 & 11 & 407 \\
\hline Total & $\mathbf{8}$ & $\mathbf{1 7 0}$ & $\mathbf{3 7}$ & $\mathbf{9 2 8}$ \\
\hline
\end{tabular}

(C) Memòria d'activitat 1996 del Servei Català de la Salut [1997, p. 24].

\subsubsection{PRESTACIÓN FARMACÉUTICA}

Los centros de atención farmacéutica se clasifican según sus funciones en:

- Oficinas de farmacia.

- Farmacias hospitalarias.

- Depósitos de medicamentos.

- Botiquines. 
TABLA 6.11. Recursos de prestación farmacéutica, RSUPC 1996.

\begin{tabular}{|l|r|r|r|r|}
\hline Región sanitaria & Oficinas & $\begin{array}{r}\text { Farmacias } \\
\text { hospitalarias }\end{array}$ & $\begin{array}{r}\text { Depósitos de } \\
\text { medicamentos }\end{array}$ & Botiquines \\
\hline Lleida & 177 & 6 & 6 & 15 \\
Tarragona & 235 & 7 & 6 & 16 \\
Tortosa & 66 & 2 & 3 & 5 \\
Girona & 278 & 10 & 9 & 10 \\
Costa de Ponent & 385 & 15 & 7 & 7 \\
Barcelonès Nord i Maresme & 195 & 8 & 4 & 0 \\
Centre & 449 & 13 & 13 & 5 \\
Barcelona Ciutat & 1.035 & 35 & 39 & 0 \\
\hline Total & $\mathbf{2 . 8 2 0}$ & $\mathbf{9 6}$ & $\mathbf{8 7}$ & $\mathbf{5 8}$ \\
\hline
\end{tabular}

(C) Memòria d'activitat 1996 del Servei Català de la Salut [1997, p. 25].

\subsubsection{ACTIVIDAD DE TRASPLANTES}

El año 1997 se cerró con un número de 235 donantes en Catalunya. Esta cifra representa un índice de donación por millón de población [pmp] de 39. La media española es de 29 pmp, mientras que de las organizaciones europeas es inferior a 20 pmp y la de los EEUU e de 21 pmp, en el año 1996 [Memòria d'activitat del Servei Català de la Salut 1997, 1998, p. 79]. Con este número de donaciones, en el año 1997 se ha podido realizar un total de 608 transplantes de órganos sólidos —riñones, hígado, corazón y páncreas-.

Se destaca, por un lado, que los índices de transplantes de riñón $-57 \mathrm{pmp}-$ y de hígado - 28 pmp - sitúan Catalunya en un lugar muy importante en el ámbito mundial. Por otro lado, se han aumentado considerablemente los transplantes de corazón y de pulmón y se ha mantenido la actividad en los transplantes de páncreas, que representa el 67\% del total del Estado.

La Unitat de Coordinació de l'Intercanvi d'Organs - UCIO— de la Organització Catalana de Trasplantaments — OCATT- coordina el intercambio de órganos entre todo el Estado y las diversas organizaciones europeas de transplantes. El balance global del intercambio de órganos pone de manifiesto el alto nivel de donaciones en Catalunya.

En 1997 se han recibido desde fuera de Catalunya 8 riñones, 4 hígados y dos corazones y se han enviados 25 riñones, 9 hígados, 5 corazones y 1 pulmón a otras comunidades autónomas y a otros países europeos. 
Tabla 6.12. Trasplantes en Catalunya en 1997.

\begin{tabular}{|lr|}
\hline & Trasplantes \\
\hline Renal & 348 \\
\hline Hepático & 175 \\
\hline Cardíaco & 45 \\
\hline De progenitores hemoptísicos & 418 \\
\hline De pulmón & 22 \\
\hline De páncreas & 18 \\
\hline De tejidos osteotendinosos & 737 \\
\hline De segmentos vasculares & 40 \\
\hline De válvulas cardíacas & 27 \\
\hline De piel & 19 \\
\hline De córnea & 789 \\
\hline
\end{tabular}

(C) Memòria d'activitat 1997 del Servei Català de la Salut [1998, p. 79].

\subsubsection{RECURSOS HUMANOS}

El 1996 las plazas de trabajo de la red sanitaria de utilización pública fueran 67.143, cifra ligeramente inferior a la de 1995, principalmente por la reducción de plazas en el ámbito de la atención hospitalaria. Igualmente, se observa un descenso de las plazas de atención primaria de la red no reformada en favor de las correlativas de la red reformada.

Las plazas correspondientes a la atención sociosanitaria y a la atención psiquiátrica se han incrementado moderadamente y también las correspondientes a la atención psiquiátrica en cifras reales, en la Región Sanitaria Tarragona no se hacen constar las plazas existentes sino las personas que trabajan equiparadas a 40 horas semanales. Con relación a plazas de trabajo de las empresas públicas, y en atención al lanzamiento de determinadas actividades, el incremento experimentado se cuantifica en torno del 45\% respecto al año 1995. 
TABLA 6.13. Plazas de trabajo de la RSUP de Catalunya, 1996.

\begin{tabular}{|c|c|c|c|c|c|c|}
\hline \multicolumn{7}{|c|}{ Atención primaria } \\
\hline Región sanitaria & $\begin{array}{r}\text { Hospitales } \\
\text { de la XHUP }\end{array}$ & $\begin{array}{r}\text { Red no } \\
\text { reformada }\end{array}$ & $\begin{array}{r}\text { Red } \\
\text { reformada }\end{array}$ & $\begin{array}{r}\text { Atención } \\
\text { socio } \\
\text { sanítaria }\end{array}$ & $\begin{array}{r}\text { Atención } \\
\text { psiquiátrica }\end{array}$ & $\begin{array}{r}\text { Empresas } \\
\text { públicas }\end{array}$ \\
\hline Lleida & 1.940 & 484 & 560 & 208 & 73 & $64^{1}$ \\
\hline Tarragona & 2.486 & 734 & 537 & 212 & $303^{4}$ & $15^{2}$ \\
\hline Tortosa & 787 & 168 & 193 & 222 & 9 & 0 \\
\hline Girona & 3.202 & 896 & 496 & 214 & 309 & $11^{3}$ \\
\hline Costa de Ponent & 6.762 & 2.179 & 1.445 & 334 & 999 & 298 \\
\hline $\begin{array}{l}\text { Barcelonès Nord i } \\
\text { Maresme }\end{array}$ & 3.642 & 1.457 & 567 & 234 & 538 & 0 \\
\hline Centre & 7.532 & 2.111 & 1.566 & 308 & 78 & 0 \\
\hline Barcelona Ciutat & 17.211 & 3.665 & 807 & 784 & 212 & 291 \\
\hline Total & 43.562 & 11.694 & 6.171 & 2.516 & 2.521 & 679 \\
\hline \multicolumn{7}{|c|}{$\begin{array}{l}\text { 1. El personal del Hospital de Santa Maria se incluye en los datos de los hosptales de la XHUP. } \\
\text { 2. Incluye las plazas de los servicios centrales y del SEMSA. } \\
\text { 3. Solamente incluye las plazas de los servicios centrales. } \\
\text { 4. Personas equiparadas a las } 40 \text { horas. }\end{array}$} \\
\hline
\end{tabular}

(C) Memòria d'activitat 1996 del Servei Català de la Salut [1997, p. 26].

\subsubsection{RECURSOS ECONÓMICOS}

El presupuesto inicial destinado a la prestación de servicios sanitarios en Catalunya para 1997 ha sido de 605.807 millones de pesetas, un 5,99\% más que en 1996.

El presupuesto consolidado final Servei Català de la Salut—Institut Català de la Salut para el 1997 fue de 633.042 millones de pesetas, lo que significa un incremento de 27.235 millones de pesetas respecto al presupuesto inicial aprobado por el Parlament de Catalunya ${ }^{4}$.

TABLA 6.14. Crecimiento \% del presupuesto SCS-ICS.

\begin{tabular}{|c|c|c|c|c|c|c|c|c|c|c|}
\hline 1986 & 1987 & 1988 & 1989 & 1990 & 1991 & 1992 & 1993 & 1994 & 1995 & 1996 \\
1987 & 1988 & 1989 & 1990 & 1991 & 1992 & 1993 & 1994 & 1995 & 1996 & 1997 \\
\hline 12,27 & 17,00 & 17,38 & 20,43 & 16,23 & 15,12 & 12,28 & 7,24 & 4,85 & 3,50 & 5,99 \\
\hline
\end{tabular}

(C) Memòria d'activitat 1997 del Servei Català de la Salut [1998, p. 101].

4 A 31 de diciembre de 1997 se tiene que diferenciar tres conceptos: el presupuesto inicial, que resulta de consolidar los presupuestos del Servei Català de la Salut y del ICS aprobados por el Parlament de Catalunya; el presupuesto final, que incluye la agregación de las variaciones de créditos que se han producido durante el ejercicio, tanto del presupuesto del Servei Català de la Salut como del ICS, y el gasto producido en el transcurso del ejercicio. 
FIGURA 6.4. Estructura económica consolidada SCS/ICS, 1997.

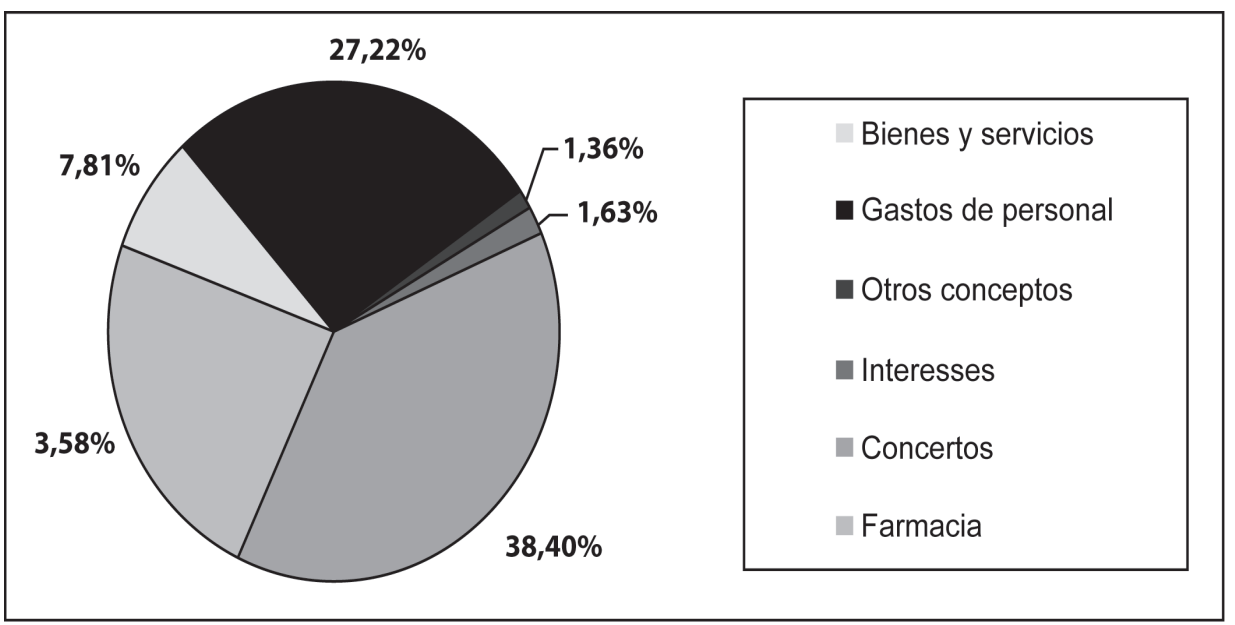

(C) Memòria d'activitat 1997 del Servei Català de la Salut [1998, p. 104].

FIGURA 6.5. Estructura funcional del presupuesto SCS/ICS, 1997.

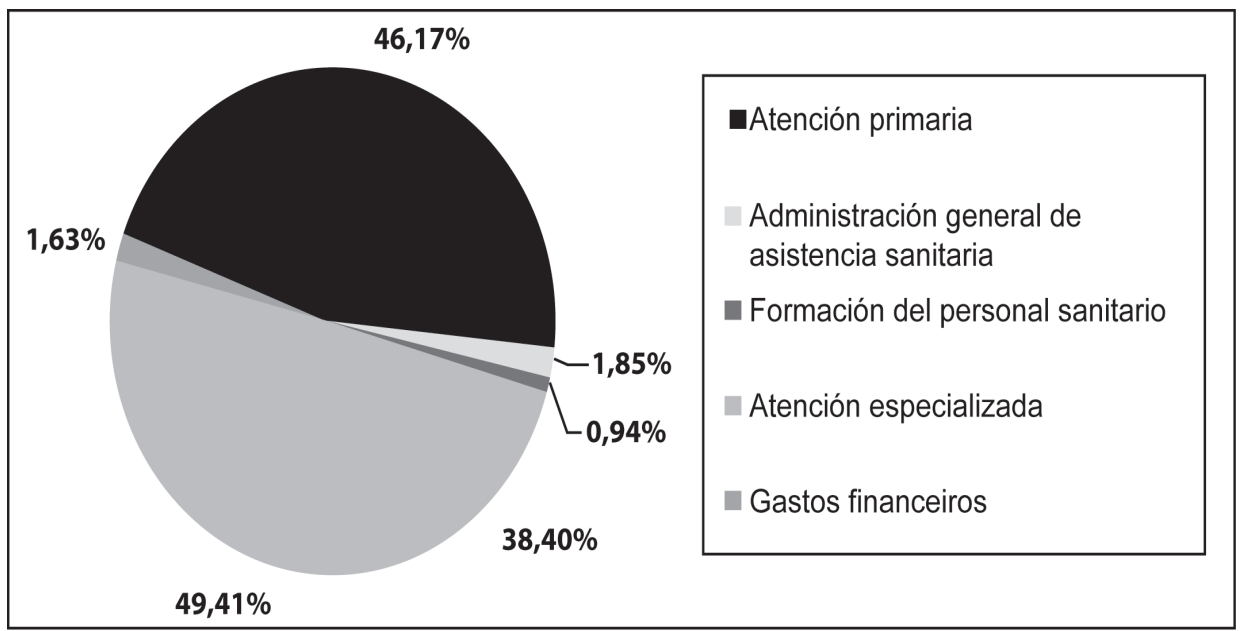

(C) Memòria d'activitat 1997 del Servei Català de la Salut [1998, p. 104]. 


\subsubsection{LA ESTRUCTURA CORPORATIVA DEL SCS}

La estructura organizativa del Servei Català de la Salut está conformada en tres ámbitos.

- Por un lado, los servicios matrices y la estructura desconcentrada territorial de regiones sanitarias y sectores sanitarios.

- En segundo lugar, las empresas públicas adscritas al Servei Català de la Salut.

- Y, finalmente, los órganos de participación comunitaria presentes en todos los niveles de la organización.

En 1993 se inicia el despliegue territorial del Servei Català de la Salut con la entrada en funcionamiento de las ocho regiones sanitarias:

- Lleida.

- Tarragona.

- Tortosa.

- Girona.

- Costa de Ponent.

- Barcelonès Nord i Maresme.

- Centre.

- Barcelona Ciutat.

La disposición adicional catorce de la LOSC, incorporada por medio de la Ley 11/1995, de 29 de septiembre, de modificación parcial de esta misma Ley, adscribe el Consorcio Sanitario de Barcelona al Servei Català de la Salut y establece que las funciones previstas para las regiones sanitarias, en relación con la ciudad de Barcelona, serán directamente asumidas por el Consorcio Sanitario de Barcelona. Para completar el despliegue de la estructura territorial, durante el 1994 se inicia la puesta en marcha de los sectores sanitarios —orden del consejero de Sanidad y Seguridad Social de 10 de mayo de 1994-. Los sectores sanitarios, tal y como prevé a la LOSC, están constituidos por diversas áreas básicas de salud y son los órganos territoriales donde se desarrollan y coordinan las actividades de prevención de la enfermedad, promoción de la salud y asistencia sanitaria en el nivel de la atención primaria y de las especialidades médicas. Las principales funciones de los sectores son el análisis de las necesidades de salud de la población y la programación, la coordinación y la evaluación de los servicios y prestaciones sanitarias y sociosanitarias, con el objetivo de garantizar a 
los usuarios del sistema sanitario público una atención efectiva y satisfactoria.

Para desarrollar estas funciones, en la modificación de la LOSC se han definido dos unidades funcionales dependientes del director del sector: El ámbito de Análisis y Programación y el ámbito de Servicio al Cliente.

El despliegue de los órganos de gestión de los sectores se está haciendo de manera progresiva para ajustarse a las disponibilidades presupuestarias, pero garantizando el desarrollo de las funciones básicas. Para hacer más eficiente esta puesta en funcionamiento se han definido las direcciones operativas, que agrupan las funciones de diversos sectores - resolución del consejero de Sanidad y Seguridad Social de 12 de mayo de 1994- A finales de 1996 el personal de la organización corporativa era de 574 personas, 220 de las cuales correspondían a las regiones y a los sectores sanitarios. La mayor parte del personal del Servei Català de la Salut proviene del Departament de Sanitat i Seguretat Social y del Institut Català de la Salut. El traspaso de personal ha sido posible por la redistribución de funciones y la creación del Servei Català de la Salut, funciones que antes eron asignadas al ICS o al mismo Departamento.

Con la entrada en funcionamiento en 1995 de dos nuevas empresas públicas - Institut Català d'Oncologia, y Serveis Sanitaris de ReferènciaCentre de Transfusió i Banc de Teixits-, ya son nueve las empresas públicas adscritas o participadas por el Servei Català de la Salut. 
FIGURA 6.6. Organigrama funcional del SCS.

\section{Estructura corporativa del SCS \\ Órganos de dirección}

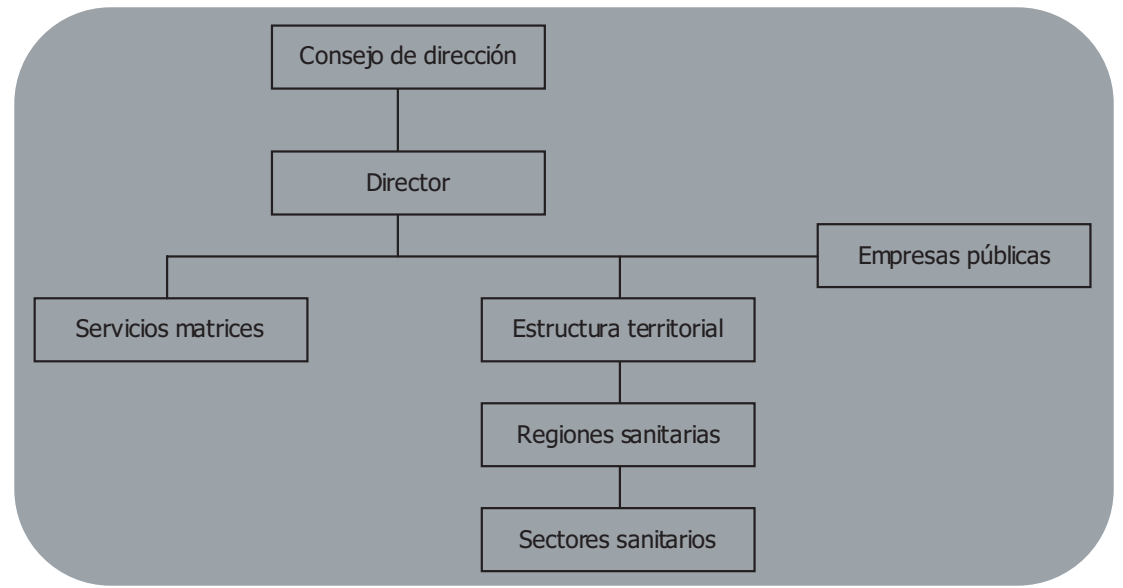

\section{Servicios matrices}

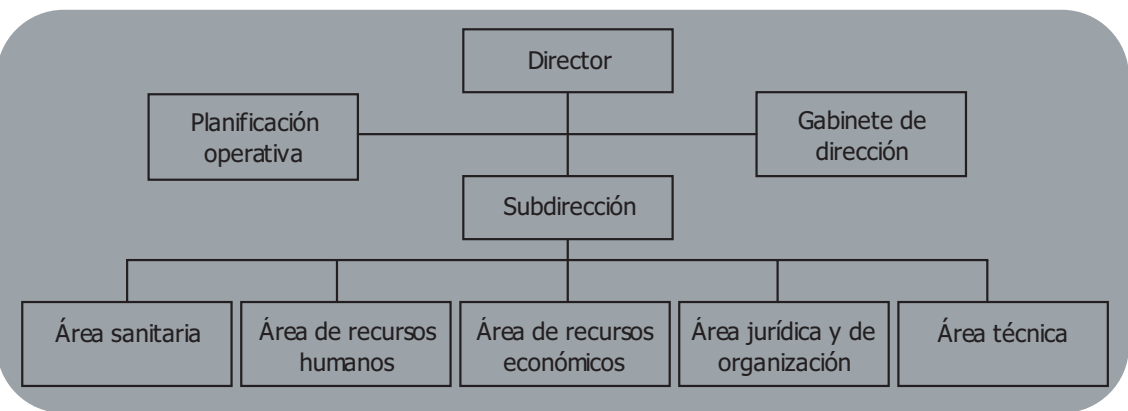

\section{Regiones sanitarias}

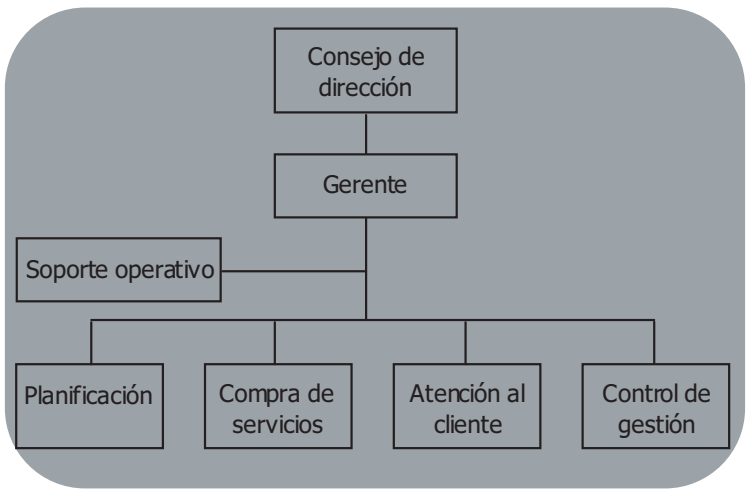

\section{Sectores sanitarios}

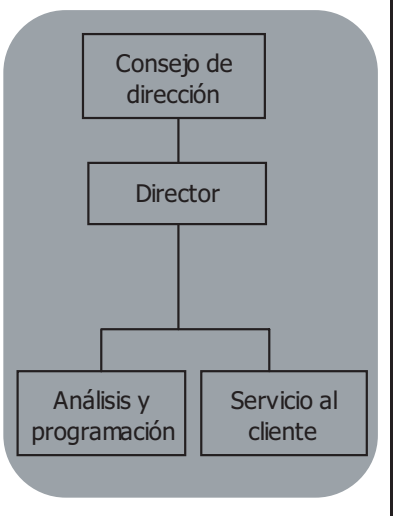

(C) Cataluña, un modelo para la sanidad pública [1997, p. 12]. 


\subsubsection{LÍNEAS ESTRATÉGICAS CORPORATIVAS}

Las líneas estratégicas corporativas son aquel conjunto de prioridades que contribuyen a la consecución de los objetivos del Servei Català de la Salut y que se desarrollan a partir de sus responsabilidades como corporación o empresa al servicio del sistema sanitario público catalán.

Estas líneas son las siguientes:

- Plan estratégico.

- El control corporativo.

- El despliegue territorial.

- La comunicación y la imagen corporativas.

- La política de recursos humanos.

- El sistema de información integrado.

- La política de infraestructuras sanitarias.

\subsubsection{PLAN ESTRATÉGICO}

El Plan estratégico es el instrumento de dirección y comunicación interna del Servei Català de la Salut.

Su objetivo es avanzar en la planificación estratégica, la dirección por objetivos, la gestión por proyectos, el control por resultados e integrar y cohesionar los equipos humanos del Servei Català de la Salut, potenciando la responsabilidad de todos en los objetivos y proyectos corporativos.

\subsubsection{CONTROL CORPORATIVO}

El sistema de control corporativo, operativo y estratégico del Servei Català de la Salut obedece a la necesidad de disponer de instrumentos actualizados para la dirección y la gestión que permitan la previsión y la planificación de las actuaciones.

El Plan anual de actividades y el Cuadro de mando son los instrumentos básicos para la dirección del Servei Català de la Salut.

El sistema de control de las empresas públicas y de los consorcios sanitarios donde la Generalidad de Catalunya tiene una participación de capital, total o mayoritaria, obedece a la necesidad de disponer de instrumentos actualizados que permitan orientar el proceso de toma de decisiones de los representantes de la Generalitat de Catalunya en sus consejos de administración. 


\subsubsection{DESPLIEGUE TERRITORIAL DEL SCS}

FIGURA 6.7. Regiones sanitarias del SCS, 1997.

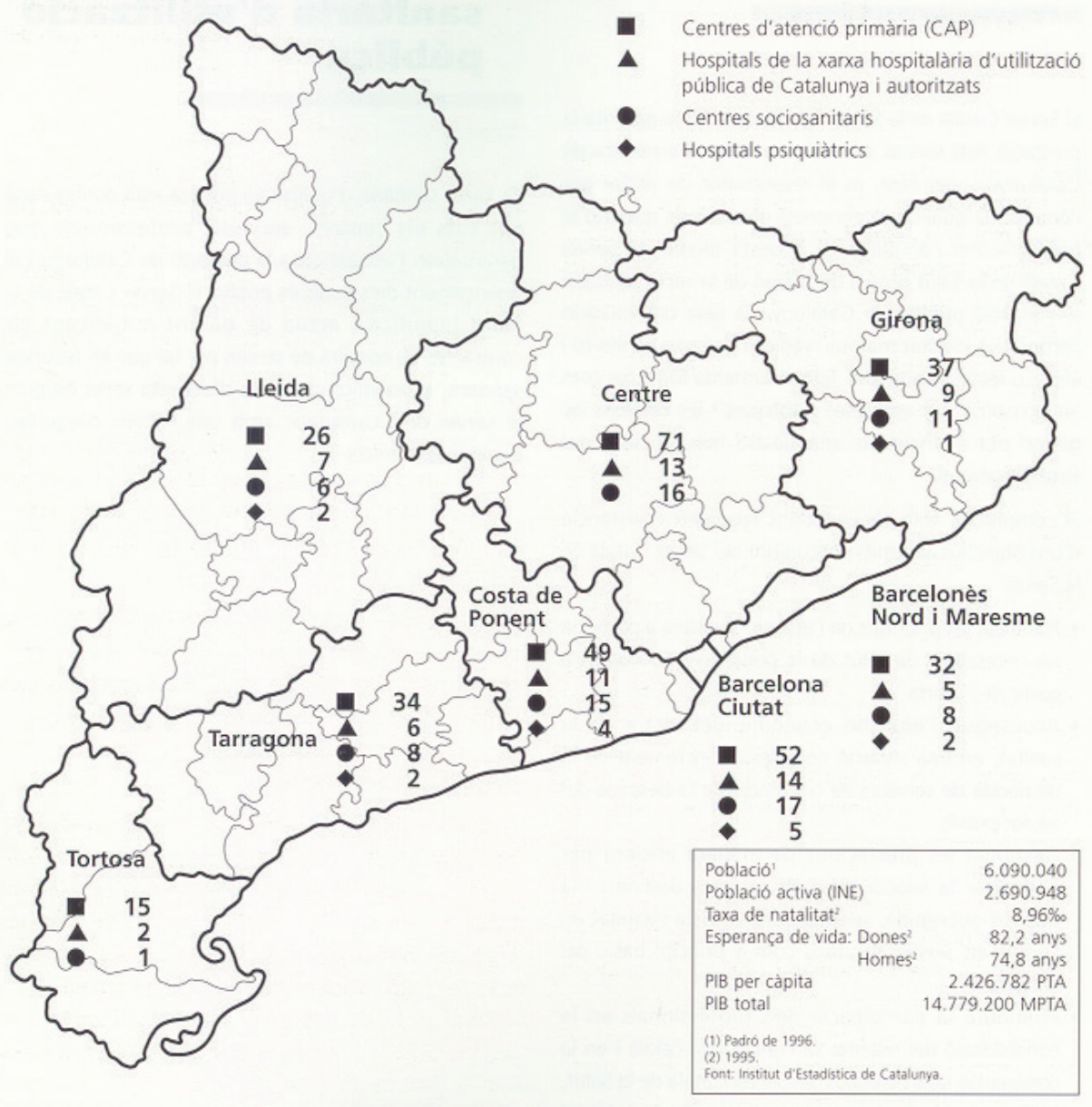

(C) Memòria d'activitat 1997 del Servei Català de la Salut [1998, p. 18].

La concepción del Servei Català de la Salut como ente profundamente desconcentrado responde a la voluntad de conocer mejor la realidad y las necesidades de salud del territorio y, por tanto, acercar las actuaciones de la Administración sanitaria al ciudadano. Una vez desplegadas las regiones sanitarias se está avanzando en el despliegue de los sectores sanitarios y de las direcciones operativas de los sectores, así como en la puesta en funcionamiento de sus órganos de dirección y participación comunitaria. 


\subsubsection{COMUNICACIÓN E IMAGEN CORPORATIVAS}

La comunicación y la imagen institucionales del Servei Català de la Salut tienen cuatro destinatarios de referencia sobre los que hay que incidir de una manera directa y específica, y que son los siguientes:

- La red sanitaria pública, como proveedora del sistema sanitario catalán.

- Los profesionales de esta red, como elemento clave en la calidad asistencial y de trato a los ciudadanos.

- Los ciudadanos, centro de todo el sistema.

- El personal de la corporación, cliente interno que ha de participar de los objetivos de la corporación.

En estas líneas estratégicas destacan la cohesión de la imagen gráfica, la dirección editorial y los instrumentos de comunicación institucional con todos los protagonistas del sistema y con los medios de comunicación, y las relaciones institucionales de ámbito nacional e internacional del modelo sanitario catalán.

La política informativa del Servei Català de la Salut al ciudadano, iniciada en el año 1995 con el objetivo de informar sobre los recursos y servicios y las vías de acceso al sistema sanitario público de Catalunya, se ha transformado en la vertiente clave de la comunicación estratégica del Servei Català de la Salut. Los principales instrumentos de comunicación de esta nueva política informativa son:

- La instalación de una placa de identificación en los centros proveedores del SCS que configuran la red sanitaria de utilización pública.

- La edición de los folletos: Al servicio de los ciudadanos. Una red sanitaria de calidad para todos. La salud, más cerca.

Folletos que se distribuyen por medio de los expositores del Servei Català de la Salut que, progresivamente, se sitúan en todos los centros de la red.

\subsubsection{FORMACIÓN CORPORATIVA}

El Plan de formación 1996, diseñado a partir del proceso de análisis de necesidades de formación que se hizo en la segunda mitad del año 1995, tiene como objetivo facilitar las herramientas y los instrumentos que permiten aumentar las habilidades y conocimientos de los profesionales de la corporación para mejorar la eficacia y la eficiencia de su trabajo.

El Plan de formación incluye las actividades de formación interna, recogidas en el programa de formación, las actividades de formación externa, que dan respuesta a necesidades específicas que no quedan cubiertas con la oferta interna y las sesiones monográficas de carácter informativo y abiertas a todo el personal sobre el Plan 
estratégico del SCS o bien sobre proyectos concretos del ámbito de la sanidad pública. El programa de formación interna del año 1996 representa el interés común de los servicios matrices y regiones sanitarias del Servei Català de la Salut en el marco de la formación centralizada que se ofrece desde los servicios matrices. También, las regiones sanitarias organizan actividades de formación específica en sus sedes, para poder hacer una oferta de formación más específica y descentralizada.

En un marco de contención del gasto, el presupuesto de formación se reduce un 35\% de media entre la formación externa y la formación interna. En el año 1996 el Plan de formación ha incluido actividades que se sitúan en el ámbito estratégico, en el ámbito del factor humano y en el ámbito técnico, y también, cursos de lengua catalana y de microinformática.

\subsubsection{SISTEMA DE INFORMACIÓN}

Disponer de un sistema que posibilite la obtención, el almacenamiento, el tratamiento y la distribución de la información necesaria para facilitar el ejercicio de las funciones asignadas al SCS, especialmente de una forma integrada, constituye una de las líneas prioritarias de actuación del SCS.

FIGURA 6.8. Sistema de información.

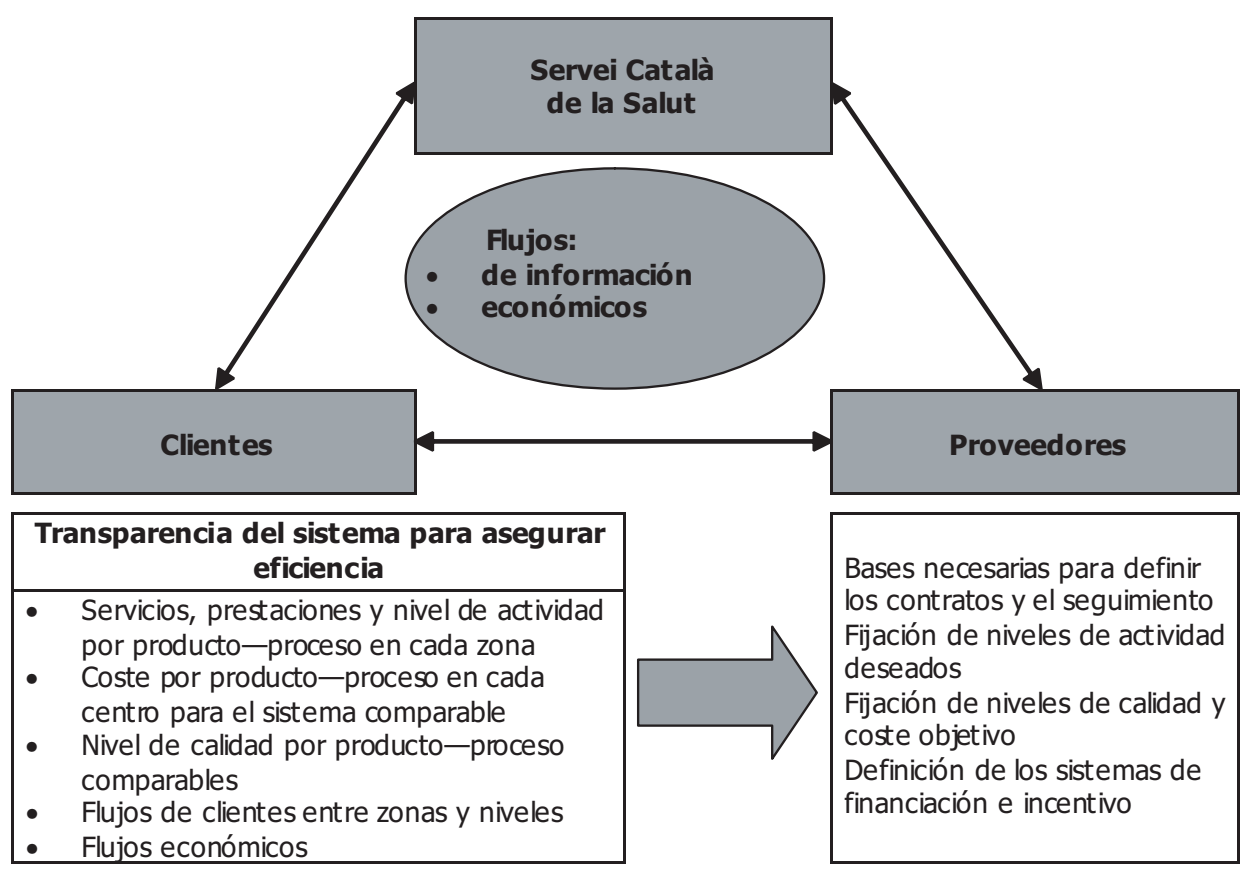

C McKinsey \& Company [1992, p. 15]. 
En este sentido, se estableció de estándares de funciones, procesos y datos que permiten la relación y la comunicación entre las entidades proveedoras de servicios sanitarios, el SCS y los usuarios del sistema sanitario público. También se destaca el establecimiento de estándares informáticos que constituyen un punto de referencia para la renovación tecnológica del instrumental informático que da soporte al despliegue del sistema de información. Este despliegue se materializa a través del conjunto de desarrollos informáticos que se ponen a disposición de la red sanitaria.

\subsubsection{ORGANIZACIÓN INSTRUMENTAL DE LA COMPRA DE SERVICIOS}

El proceso de contratación de servicios que realiza el Servei Català de la Salut tiene su origen en las necesidades de salud de la población, detectadas por el Plan de salud.

FIGURA 6.9. Proceso para la contratación de servicios.

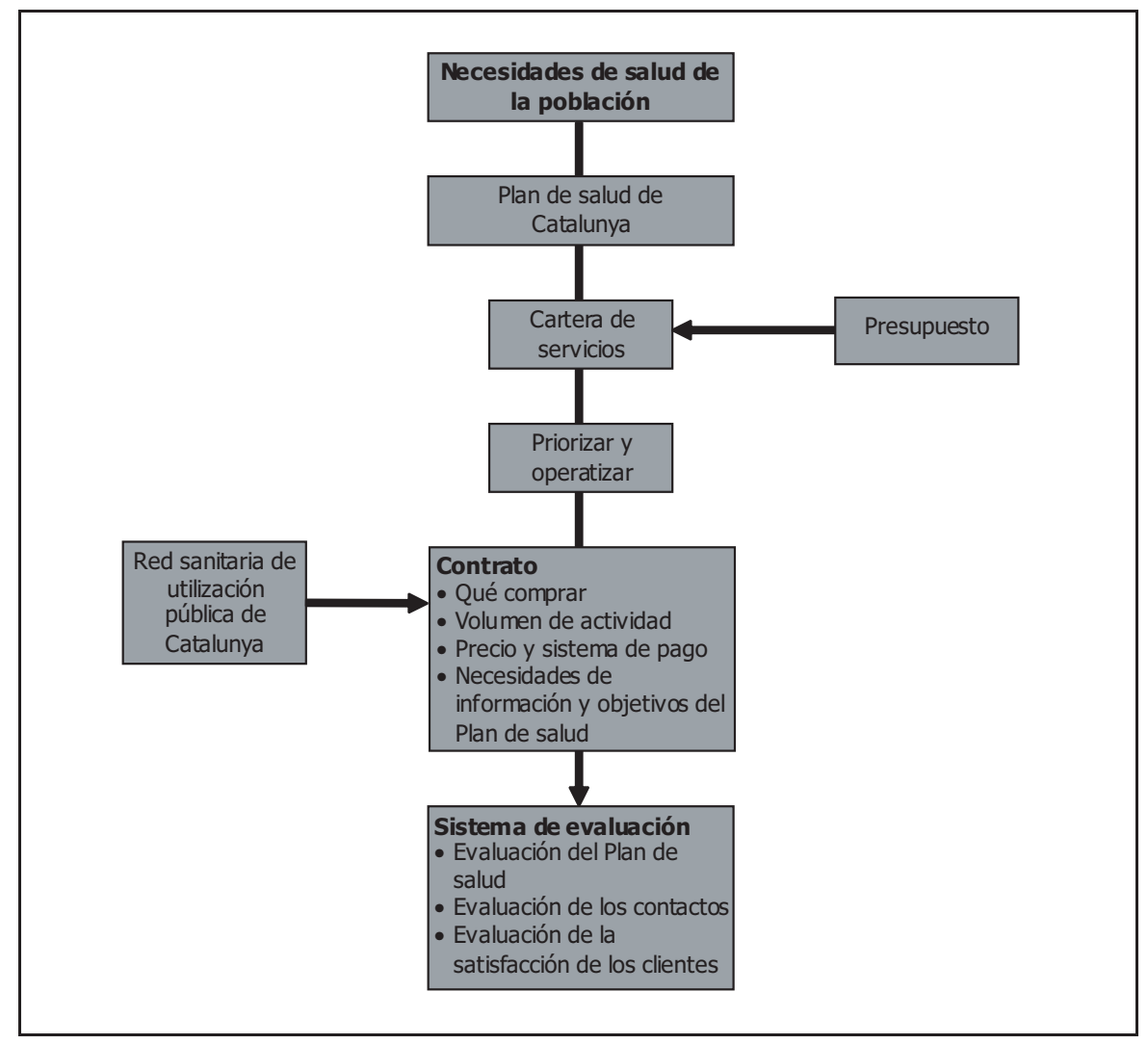

(C) Cataluña, un modelo para la sanidad pública [1997, p. 19]. 
A partir de esta información, y de acuerdo con el catálogo de prestaciones del Sistema Nacional de Salud, se define la cartera de servicios del Servei Català de la Salut. Es decir, todas aquellas prestaciones que se financian con el presupuesto público.

Una vez conocidas estas informaciones, la relación del Servei Català de la Salut con las entidades proveedoras se vincula a través de los contratos, en los que se recogen los objetivos de salud, los servicios que se contratan, el precio y el sistema de pago, y los requerimientos de información que hay que aportar al sistema sanitario.

FIGURA 6.10. Implicaciones globales: SCS, proveedores y su interrelación.

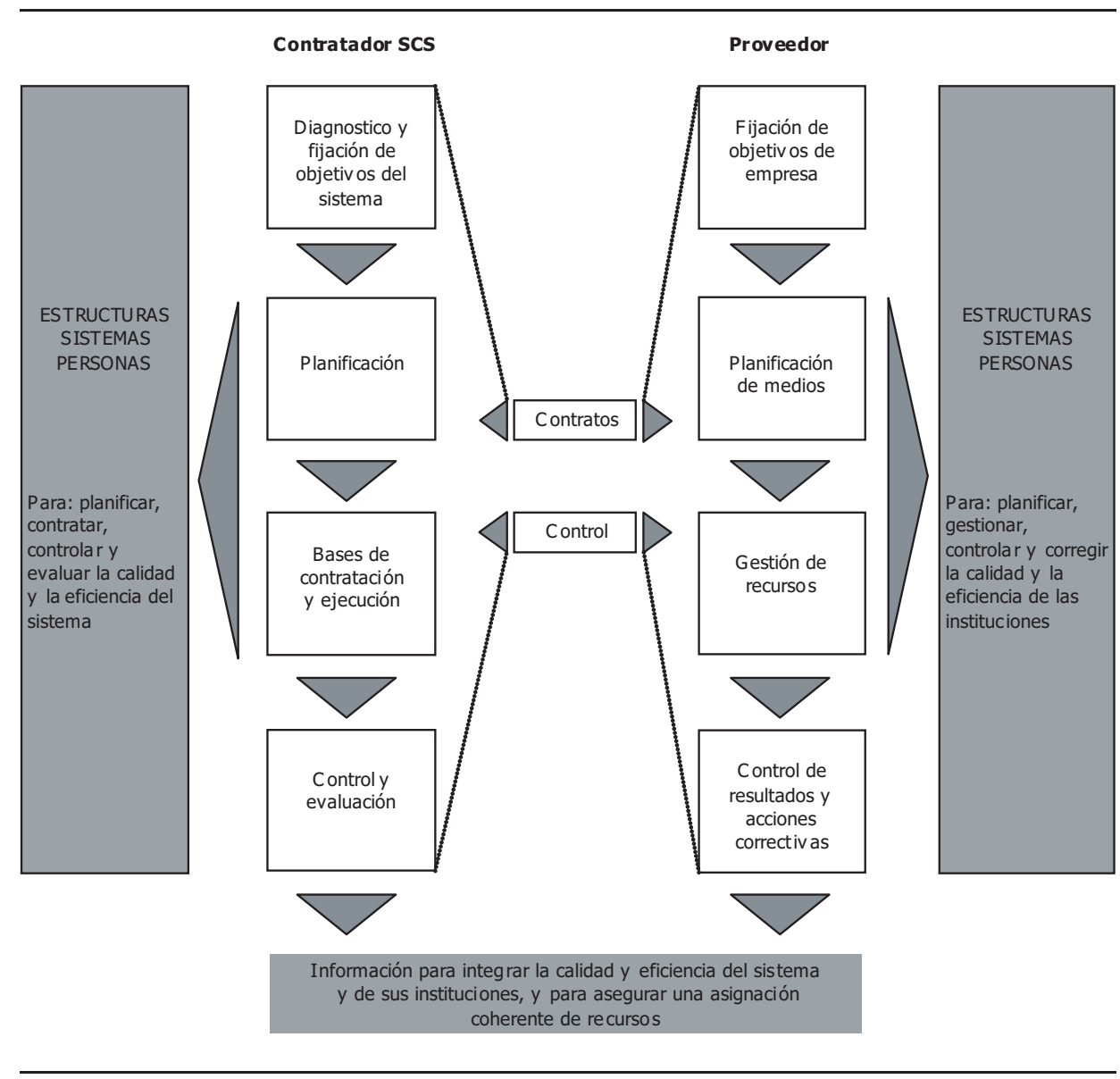

(C) McKinsey \& Company [1992, p. 12]. 
Finalmente, la evaluación permite reorientar el proceso de toma de decisiones a partir del análisis del cumplimiento de los contratos, del seguimiento del Plan de salud y del grado de satisfacción del cliente.

Uno de los instrumentos esenciales para poder conseguir una contratación eficiente es el sistema de información integrado, que permite que todo el proceso se base en un conocimiento fiable de la realidad.

El sistema de información corporativo, el sistema de información de la atención hospitalaria y de la atención primaria y las fuentes de información económica financiera y de actividad de los centros, son los principales ejes de este sistema.

Así mismo, las regiones sanitarias, como parte de la organización más cercana a las necesidades de salud de la población, tienen la responsabilidad del proceso de compra de servicios en su ámbito territorial.

Hacen a través de la negociación de los contratos, de las propuestas de contenido y de gestión de éstos y en las fases siguientes de control y evaluación.

\subsection{INSTITUT CATALÀ DE LA SALUT}

\subsubsection{POLÍTICAS Y ESTRATEGIAS CORPORATIVAS}

El Institut Català de la Salut —ICS — está haciendo un gran esfuerzo para transformar sus estructuras y sus sistemas de gestión. La orientación de esta transformación se basa en la consecución de unos resultados que permitan situar el ICS en una posición sólida dentro del conjunto del sistema sanitario. Las principales características que configuran el marco de referencia para alcanzar los objetivos $\operatorname{son}^{5}$ :

1. Calidad técnica y de servicio.

2. Eficiencia en los costes.

3. Provisión de servicios a diversos compradores.

4. Equilibrio económico financiero.

5. Integración del personal en un proyecto compartido de institución prestadora de servicios públicos sanitarios.

6. Imagen externa de grupo moderno, avanzado y líder.

5 Siguiendo el Anuari 1996, Istitut Català de la Salut [1997, p. 10]. 


\subsubsection{EL MARCO PRESUPUESTARIO}

Las políticas y los objetivos corporativos del ICS están ligados a los aspectos relativos al entorno. Un de los aspectos es el económico-financiero, que continua siendo muy restrictivo. Así lo exigen las políticas de control del déficit público y de la convergencia económica europea hacia la unión política y monetaria. Esta situación de limitación de recursos ya se asumió como una realidad de carácter estructural y, por lo tanto, las expectativas de crecimiento han de apuntar hacia fuentes alternativas de financiación. En esta situación el ICS aplica un mayor rigor presupuestario y busca oportunidades para la racionalización del gasto y la mejoría de la gestión económica.

\subsubsection{LA COMPETITIVIDAD}

El avance hacia un nuevo modelo de organización más competitivo en la provisión de servicios sanitarios requiere, además de la mejoría generalizada de los objetivos corporativos, una mejoría en los ámbitos que los usuarios y la sociedad demandan. El papel de gran proveedor de servicios sanitarios en Catalunya obliga el ICS a ser competitivo en relación con los costes de producción. Unos de los aspectos que más ventajas pueden ofrecer al grupo ICS se deriva de la coordinación de la atención primaria con la atención hospitalaria, buscando el máximo provecho de las sinergia del grupo. En este sentido, se avanza especialmente en ${ }^{6}$ :

- La integración de la actividad especializada de atención primaria en los hospitales.

- La gestión conjunta de los laboratorios de los hospitales y de la red de atención primaria.

- La coordinación funcional entre los PADES — programas de atención domiciliaria, equipos de apoyo_ y las UFISS — unidad funcional interdisciplinaria sociosanitaria-.

Otros ámbitos donde el ICS avanza en competitividad son la gestión de compras conjuntas — tanto en los hospitales como en la atención primaria—, el Plan director de servicios generales de los hospitales y la consolidación de las fundaciones como elemento para la potenciación de la investigación y la docencia.

6 Siguiendo el Anuari 1996, Istitut Català de la Salut [1997, p. 11]. 


\subsubsection{POLÍTICAS EN EL ÁMBITO DE LA ATENCIÓN PRIMARIA}

Las políticas que el ICS desarrolla en el ámbito de la atención primaria están enmarcadas en el proceso de cambio y en la necesidad de dar respuesta a las demandas de la población. Los grandes objetivos de estas políticas son?:

1. Incrementar la calidad, mejorando la accesibilidad, los sistemas de registro y de información y la prescripción farmacéutica.

2. Gestionar de manera eficiente y eficaz los recursos, con actuaciones relacionadas con las reestructuraciones y las nuevas herramientas de gestión, las políticas de las direcciones de atención primaria, la prescripción farmacéutica y las políticas de personal.

3. Transmitir una determinada cultura de empresa, con objetivos relativos a la oferta de líneas de negocio, la aplicación de la política de comunicación, la investigación y la docencia, y la imagen externa.

4. Enfocar la gestión al producto, determinando la cartera de servicios que se han de prestar y los que se han de derivar a otros niveles de la atención para mejorar el nivel de resolución.

5. Fomentar la descentralización y la responsabilidad a partir del principio de independencia de gestión y de responsabilidad sobre los resultados.

En los últimos años se implantó el trabajo por objetivos —DPO— en todos los ámbitos territoriales del ICS.

Las experiencias, en su conjunto, se valoran como positivas y la Divisió d'Atenció Primària —DAP- desea implantar esta dinámica de la organización del trabajo en todos los servicios de atención primaria. Todas las direcciones de atención primaria y los EAP disponen de una metodología homogénea que permite impulsar unas directrices comunes para todo el ámbito territorial en forma de objetivos. Los coordinadores de los EAP son los responsables de los contenidos de la DPO pactados con el director de la DAP, con la implicación de otros miembros del EAP. Se clasificaron tres tipos de objetivos ${ }^{8}$ :

1. Objetivo A, objetivos comunes. Son los objetivos comunes mínimos definidos desde la DAP y que todos los EAP se han de fijar para conseguirlos en el período determinado.

\footnotetext{
7 Siguiendo el Anuari 1996, Istitut Català de la Salut [1997, p. 11].

8 Siguiendo el Anuari 1996, Istitut Català de la Salut [1997, p. 12].
} 
2. Objetivo B, objetivos básicos. Son aquellos objetivos que la DAP considera importantes y los define con la finalidad de que sean motivo de reflexión para los EAP y base de negociación con el director de la DAP.

3. Objetivo C, objetivos óptimos. Son definidos, a partir de experiencias previas, por los respectivos EAP, con la consideración de que son oportunos y necesarios para una buena parte del EAP.

Los objetivos se clasifican en siete apartados:

1. Objetivos de salud.

2. Mejoría del nivel de resolución.

3. Mejoría en la utilización de los recursos disponibles —eficiencia-.

4. Mejoría en la calidad de los servicios y de la satisfacción de los usuarios — calidad percibida-.

5. Formación, docencia e investigación.

6. Coordinación y comunicación.

7. Sistemas de información.

\subsubsection{POLÍTICAS EN EL ÁMBITO DE LA ATENCIÓN HOSPITALARIA}

Los esfuerzos que está haciendo el ICS para transformar sus estructuras y sus sistemas de gestión queda reflejado en unas características de su nueva política corporativa.

Estas características se reflejan en nueve políticas corporativas?:

- Autofinanciación.

- Competitividad en costes y eficiencia de producción.

- Instrumentos y sistemas de gestión.

- Modelo organizativo hospitalario.

- Calidad.

- Satisfacción e integración del personal.

- Proyección de imagen.

- Oferta de servicios.

- Desarrollo corporativo.

El balance de 1996 respecto al cumplimiento de los objetivos fijados para estas políticas es globalmente positivo y, especialmente, muy bueno en lo referente a los indicadores de actividad y autofinanciación.

9 Siguiendo el Anuari 1996, Institut Català de la Salut [1997, p. 13]. 
En los últimos años el avance en los objetivos permitió que el grupo hospitalario haya progresado hacia una posición competitiva más favorable y hacia un planteamiento de futuro ajustado a los retos estratégicos que se presentan, aunque el entorno económico ha estado complejo y presenta dificultades.

En el apartado de instrumentos y sistemas de gestión se avanzó en la implantación de la contabilidad financiera para poder disponer de balances y cuentas de explotación, uno de los requisitos básicos para poder hacer frente a los próximos cambios jurídicos. Actualmente todos los hospitales disponen de estos estados contables, lo que les permiten ser auditados.

En el ámbito de la gestión asistencial se continua extendiendo el uso de las herramientas de «case mix» en el diálogo clínico, ligándolas a los costes hospitalarios. Todos los centros trabajan con los sistemas de información más utilizados — GRD, grupos relacionados con el diagnóstico, o PMC, categorías de gestión de pacientes- Cuanto al modelo organizativo hospitalario, permite introducir elementos que favorecen la gestión descentralizada y la corresponsabilidad en todos los niveles, se han establecido en todos los centros el sistema de fijación y evaluación de objetivos y se ha producido un arraigo de la cultura del trabajo por objetivos.

El1996 se han puesto en marcha nuevas experiencias de gestión descentralizada de los presupuestos - como el laboratorio y las prótesis - y algunas experiencias innovadoras de provisión — gestión de casos, implementación de protocolos y descripción y análisis de tareas-.

Las políticas de calidad han avanzado de manera importante y han permitido consolidar el concepto de calidad integral a partir de la introducción del concepto de calidad en las otras políticas y la incorporación de los indicadores de calidad en el cuaderno de mando del grupo hospitalario.

También se han facilitado los intercambios de experiencias entre centros, siguiendo la filosofía del «benchmarking».

En las políticas de personal, enderezadas a la satisfacción y a su integración, se adoptaron actuaciones tendentes a la mejoría de la identificación del personal con el centro. Se fomentó el proceso de descripción de los sitios de trabajo, que permite una mejor identificación de los profesionales con el lugar de trabajo y la adecuación de la formación y la movilidad interna, además de la mejoría de los procesos de selección. También se hizo el despliegue de la normativa sobre seguridad y salud laboral, que se desarrolló durante el 1997.

En relación con la gestión de la comunicación y con la proyección de la imagen de los hospitales del grupo ICS, se ha trabajado en acciones de refuerzo 
de la imagen y en el desarrollo de la comunicación con los usuarios. También se mejoro la señalización, tanto la interna de los centros como la urbana para acceder a ellos.

En la oferta de servicios se destaca la coordinación con la atención primaria. Especialmente en los aspectos relativos a la integración de la actividad especializada de primaria en los hospitales, orientada a garantizar la continuidad asistencial — también potenciada con otros hospitales-, incrementando la calidad y la eficiencia del proceso asistencial, aumentando la accesibilidad de los ciudadanos, desplegando nuevos servicios y motivando los profesionales.

También fue importante la potenciación de la coordinación funcional entre los equipos de soporte domiciliar - PADES— de la atención primaria y los UFISS —unidad funcional interdisciplinar sociosanitaria- de los hospitales y la gestión conjunta de los laboratorios de los hospitales y de la red de atención primaria.

En el ámbito más específicamente hospitalario, se potenció el drenaje de enfermos crónicos, potenciando las UFISS y los PADES, favoreciendo la rehabilitación domiciliaria y a través de acuerdos con otros centros sanitarios.

Un otro punto de avance fue la consolidación de un programa de atención para las urgencias del invierno, especialmente significativo en los hospitales de la Vall d'Hebron a través de la Unitat de Coordinació Assistencial.

Con relación al desarrollo corporativo, que permite una gestión ágil y de características empresariales, se efectuaron las acciones del Plan director de servicios generales con mejorías ligadas a la contención de costes de funcionamiento y mejorías en la prestación de los servicios a los usuarios —menú optativo, limpieza, lavado de ropa, etc.-.

El 1996 se consolida las empresas públicas sometidas al derecho privado - Institut Català d'Oncologia, y Centre de Transfusions i Banc de Teixits-, que han hecho crecer el ICS con más flexibilidad de gestión y una mejoría de los resultados.

También son significativas las funciones que realizan las fundaciones ligadas a los hospitales en el ámbito de la financiación de la investigación, la organización de cursos y congresos y la concesión de becas, entre otras actividades.

Para los próximos años serán elementos clave los objetivos relacionados con el proceso de cambio jurídico del ICS, la adaptación al nuevo sistema de pago del Servei Català de la Salut, la adecuación de las listas de espera a las 
expectativas de los usuarios, el liderazgo de los hospitales en el entorno de su región sanitaria, la participación de los profesionales en las decisiones evaluadoras del sistema de objetivos y, finalmente, la contención de costes por medio de medidas de microgestión.

\subsubsection{SERVICIOS CORPORATIVOS}

El centro corporativo del ICS está compuesto por:

- Gerencia.

- Dirección de organización, recursos humanos y asuntos económicos.

Incluye cuatro unidades que tienen como ámbito de responsabilidad:

- La gestión económica administrativa.

- La gestión de recursos humanos.

- El control de gestión y la planificación estratégica.

- La organización y los sistemas de información.

- División hospitalaria.

- División de atención primaria. Incluye:

- Las cuatro subdivisiones de atención primaria.

- Asesoría jurídica.

- Gabinete de comunicación.

- Atención al usuario.

- Secretaria técnica.

- Centro de reconocimiento y evaluación médica. 
FIGURA 6.11. Estructura corporativa del ICS.

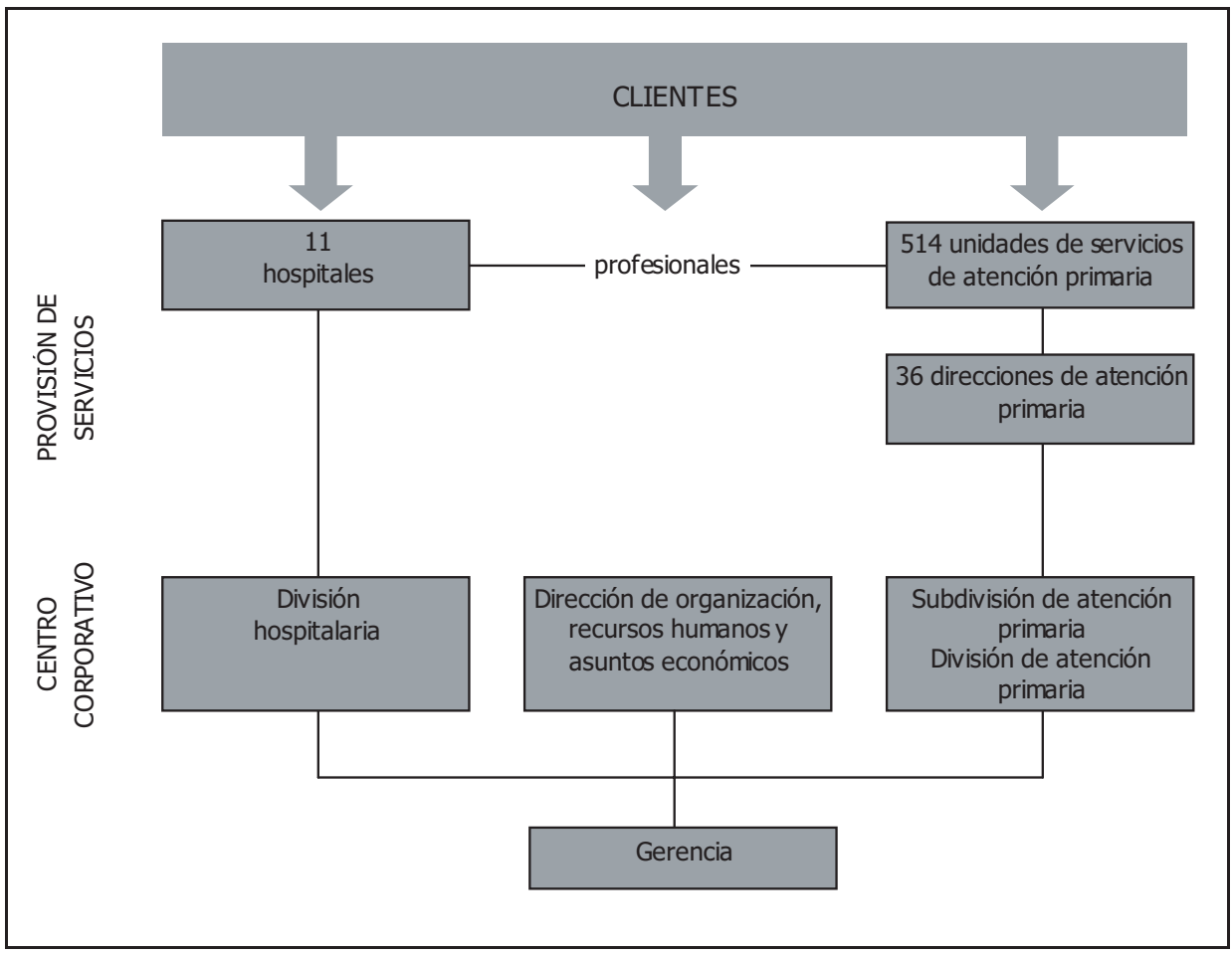

(C) Anuari 1996, Institut Català de la Salut [1997, p. 17].

El centro corporativo del grupo ICS tiene como misión fundamental dar soporte al conjunto de unidades de provisión que tienen responsabilidades descentralizadas.

El centro corporativo se orienta como un instrumento de fijación de la dirección estratégica del grupo, marcando las políticas generales y liderando el proceso de cambio en el cual el ICS se encuentra inmerso desde que la LOSC lo situó en un plan de igualdad con el resto de los proveedores del sistema público asistencial.

\subsubsection{LÍNEAS DE ACTUACIÓN DE CALIDAD}

La calidad técnica de la asistencia que ofrecen los centros del ICS constituye uno de los activos de la organización y un indudable punto fuerte que los sitúa en una posición de liderato respecto a los competidores.

En los últimos años han aumentado significativamente las expectativas de los ciudadanos que, además de la calidad técnica, piden una atención personalizada 
que cubra plenamente sus necesidades. En este sentido, las mejorías que se han logrado durante los últimos años han consolidado las estructuras existentes de los programas de calidad en todos los hospitales y se han realizado proyectos de mejoría en áreas clínicas y de satisfacción de los pacientes.

El conjunto de hospitales ha iniciado cerca de 325 acciones de mejoría, entre las que hay que destacar las relacionadas con la coordinación de la atención primaria y de las consultas externas. Como nuevos temas importantes hay que destacar la acogida y la información al paciente y a la familia en las áreas de urgencias y la mejoría de la gestión de las listas de espera.

Los centros que constituyen el grupo ICS tienen como misión la docencia, la formación y la investigación. Estos objetivos forman parte esencial de la cultura del grupo. La investigación es una actividad esencial que desarrollan los centros del ICS. Tanto en los centros hospitalarios como en los centros de atención primaria hay unidades de investigación acreditadas por el Departament de Sanitat i Seguretat Social.

Además, hay cinco unidades de investigación integradas en la Red Estatal de Unidades de Investigación. Recientemente se ha abierto una nueva etapa marcada por la necesidad de una adecuada gestión, promoción, difusión y potenciación de la investigación, a la que se ha respondido con la creación de fundaciones en los centros hospitalarios. En el ámbito de la formación de pregraduación, el ICS ofrece en sus centros hospitalarios y de atención primaria formación de la licenciatura en Medicina general y de las carreras de Enfermería y de Asistencia social. Cada año unos 300 licenciados en Medicina general se titulan en centros del ICS. En el ámbito de la formación de postgraduación, el ICS colabora con la formación de médicos y comadronas internas residentes — MIR y CIR—. La formación continuada se lleva a cabo en la práctica diaria a través de las sesiones clínicas, teóricas y bibliográficas y con los programas de actividades específicas de formación de cada centro.

\subsection{INSTITUT D'ESTUDIS DE LA SALUT}

El Institut d'Estudis de la Salut —IES—, creado por el Decreto de 25 de febrero de 1980, convalidado por la Ley 2/1981, de 22 de abril y posteriormente modificado por el Decreto 324/1990, de 21 de diciembre, es un organismo autónomo de la Generalitat de Catalunya adscrito al Departament de Sanitat i Seguretat Social, responsable de la formación de los profesionales que trabajan en el sistema sanitario, y de la investigación sanitaria en Catalunya. 
De acuerdo con estas funciones y responsabilidades desarrolla actividades docentes, de investigación educativa en ciencias de la salud y de debate técnico, así como actividades en el ámbito nacional e internacional.

El IES tiene como misión identificar las necesidades de formación e investigación en ciencias de la salud del sistema sanitario y de las políticas sanitarias y fomentar la implementación de las posibles respuestas. Asesorar y dar soporte a las instituciones sanitarias en los ámbitos de la formación y el desarrollo de su personal y ser una herramienta de carácter intersectorial facilitadora de los cambios de la cultura del trabajo fundamentalmente de la innovación educativa.

Sus funciones, sin prejuicio de aquellas otras atribuidas a diferentes órganos y entidades de la Generalitat, son las siguientes ${ }^{10}$ :

1. Formar, reciclary perfeccionar de manera continuada los profesionales sanitarios y no sanitarios del campo de la salud y de la gestión y la administración sanitarias desde una perspectiva interdisciplinaria.

2. Asesorar los departamentos de la Generalitat y el Servei Català de la Salut en todos los asuntos que le sean consultados.

3. Dar soporte a las políticas sanitarias a través de la realización de actividades formativas y de investigación.

4. Explorar y aplicar nuevas metodologías pedagógicas y evaluadoras para los diferentes niveles de formación — pregraduación, postgraduación y formación continuada- de los profesionales sanitarios.

5. Colaborar con las instituciones responsables de la formación de pregraduación y postgraduación de los profesionales sanitarios.

6. Fomentar la creación de interrelaciones en el ámbito del desarrollo profesional.

7. Fomentar la capacidad de prestar cuentas a la sociedad de las organizaciones por medio del desarrollo de sistemas evaluadores.

8. Asesorar en la fijación de la política de investigación y en el establecimiento de prioridades con relación a la investigación en materia de salud.

9. Fomentar y coordinar programas de investigación y estudios en ciencias de la salud.

10 Siguiendo parcialmente la Memòria del Departament de Sanitat i Seguretat Social 1997 [1998, p. 148]. 
10. Planificar y promover la investigación en relación con los problemas y las necesidades de salud de la población de Catalunya.

11. Fomentar estudios y investigaciones en el ámbito de la formación de los profesionales sanitarios —especialistas en ciencias de la salud-.

12. Estudiar las tendencias en las enfermedades cardiovasculares en Catalunya.

Para cumplir con esta misión y las funciones descritas, el IES desarrolla anualmente diversas acciones en el ámbito de la formación, de la investigación educativa, de la evaluación del personal sanitario y en la definición de las competencias profesionales.

Los ámbitos prioritarios del programa anual incluyen actividades docentes para el desarrollo profesional y de las organizaciones — formación de los formadores-, la salud mental y las drogodependencias, la salud pública, la atención primaria de salud, la atención sociosanitaria, la mejora de la calidad asistencial, la formación de postgraduación, la mejora de la comunicación y relación asistencial de los profesionales sanitarios con los usuarios y pacientes, y la formación en investigación, entre otros.

FIGURA 6.12. Organigrama del IES.

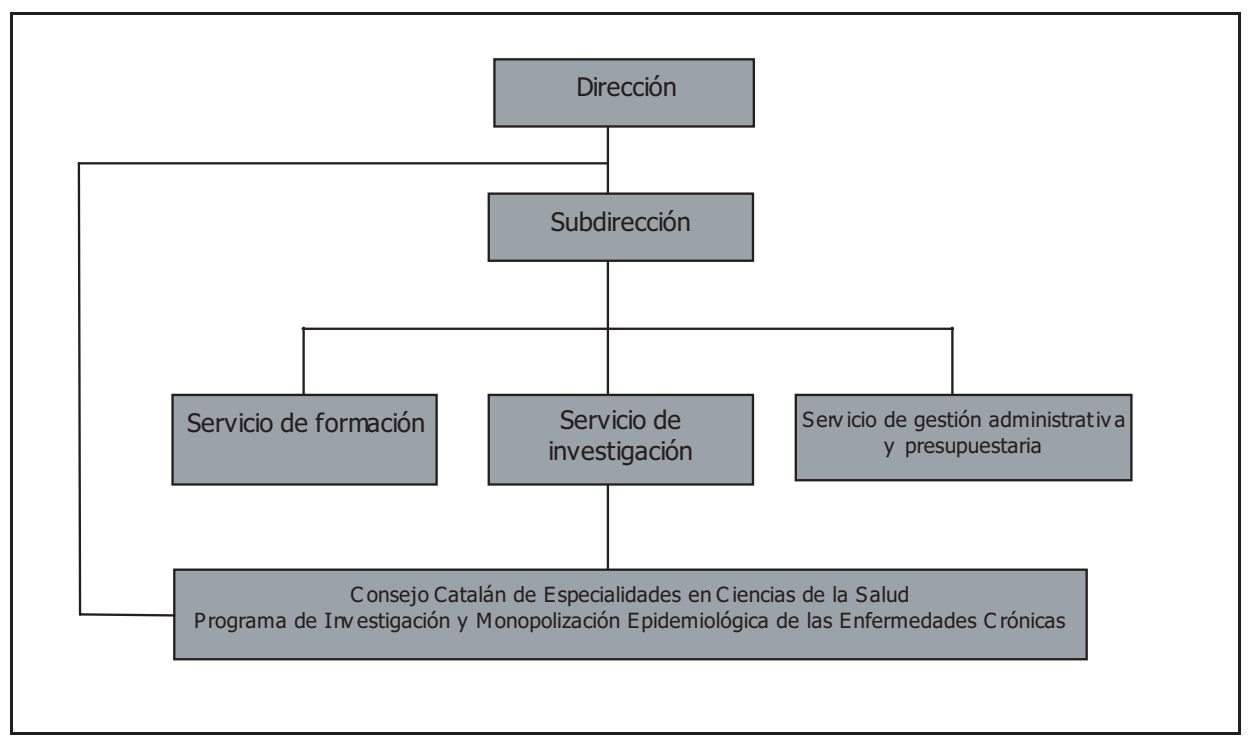

(C) Memòria del Departament de Sanitat i Seguretat Social 1997 [1998, p. 147]. 
Además, el IES, proporciona asesoramiento docente a los centros sanitarios para la planificación, organización y evaluación de sus programas formativos.

Con respecto a la evaluación de las competencias profesionales, se han desarrollado diversos proyectos evaluadores de los profesionales en pregraduación — medicina y enfermería - postgraduación — medicina interna, medicina familiar y comunitaria, pediatría, neonatología y comadronas - y en el diseño de un modelo de evaluación para el incentivo y promoción de los profesionales en los centros sanitarios, juntamente con el área de Recursos Humanos del Servei Català de la Salut.

En relación con la definición de las competencias profesionales, el Consell Català d'Especialitats en Cièncias de la Salut —CCECS - realizó diversos estudios, entre ellos se destaca la elaboración de un documento sobre las competencias del personal de enfermería, el desarrollo sobre la habilidad o áreas de capacitación específica y el estudio sobre geriatría, entre de otros.

Para cumplir con el objetivo de innovación que pretende, el IES mantiene relaciones con instituciones internacionales para incorporar los avances científicos en los ámbitos de su competencia. Las actividades desarrolladas por el Programa de Investigación y Monitorización Epidemiológica de las Enfermedades Crónicas, en el examen encuesta de salud y por el Registro de Infarto Agudo de Miocardio, que forma parte de un programa conjunto de diversos países — Proyecto MONICA—.

\subsubsection{ACTIVIDADES DE FORMACIÓN Y DOCENCIA}

La principal misión del Servicio de Formación es promover la formación continuada entre los profesionales del ámbito sanitario. La formación se entiende en el sentido de facilitar el aprendizaje desde la propia experiencia, de manera que potencia la idea de que cada centro o institución organiza sus actividades en función de las posibilidades de aprovechar los propios recursos y de aprender a organizarse para facilitar el aprendizaje. El Servicio de Formación, en este sentido, desarrolla formación específica para los docentes y responsables de formación, y desarrolla actividades en los ámbitos que son más innovadores y tienen relación con la mejoría de la práctica profesional — comunicación, educación sanitaria y mejoría de la calidad-.

Por otro lado, se sigue llevando a cabo aquellas actividades solicitadas por las diferentes unidades del Departament de Sanitat i Seguretat Social y del Servei Català de la Salut con el objetivo de transmitir a los profesionales los mensajes 
que se desprenden de las diversas políticas desarrolladas, como es el caso de Salud Mental, Atención Primaria de Salud, el órgano Técnico de Drogodependencias, y los programas de la SIDA y de Vida a los Años. El IES participa en un abanico de iniciativas con el objetivo de mejorar la formación de postgraduación, y en este marco tiene la responsabilidad de determinadas actividades. Junto con el área de Recursos Humanos del Servei Català de la Salut inició un proceso de visitas a los gerentes y directores médicos de los centros acreditados de las regiones sanitarias.

El IES proporciona soporte al Programa Común Complementario que se ha iniciado en su fase piloto y que incluye cuatro centros acreditados y dos unidades docentes de medicina familiar y comunitaria. Con relación a la tarea de tutoría en la formación de postgraduación, se han encaminado dos tipos de actuaciones. Una de ellas consiste en sesiones de trabajo para los tutores de los hospitales acreditados para la formación de postgraduación que cubre toda Catalunya. La otra consiste en la organización de seminarios destinados a los tutores que ya han participado en la realización de la $1^{a}$ edición del Programa de desarrollo docente para tutores. El IES está representado en la Comisión Permanente de la Red de Comisiones de Docencia y Asesores.

\subsubsection{EVALUACIÓN DE LAS COMPETENCIAS PROFESIONALES}

En relación con las funciones y responsabilidades del IES en la formación y reciclamiento del personal sanitario, y después de haber detectado vacíos en los diferentes niveles de la formación de pregraduación, postgraduación y formación continuada de los médicos, se iniciaron en 1994, en colaboración con instituciones extranjeras expertas, un conjunto de acciones para definir las competencias de los médicos en los niveles citados y diseñar instrumentos de evaluación.

Los diversos proyectos iniciados se han realizado con la colaboración de las instituciones catalanas responsables de la formación de pregraduación —Facultades de Medicina y Escuelas de Enfermería- y postgraduación — sociedades científicas de la Academia de Ciencias Médicas de Catalunya y de Baleares-. El objetivo genérico de todos los proyectos realizados es dar información sobre la formación recibida por los profesionales tanto en pregraduación como en postgraduación, para que se puedan introducir los cambios necesarios. Para desarrollar estos proyectos, el IES está colaborando con el Centre d'Evaluation des Sciences de la Santé —CESSUL— de l'Université du Laval —Québec_- con los comités de expertos de las sociedades catalanas 
de Medicina Interna, de Medicina Familiar y Comunitaria, de Pediatría y de la Escuela de Comadronas. Por otro lado, expertos catalanes del Grupo de Formación y Evaluación de las Competencias Clínicas están colaborando con instituciones internacionales -Educational Comision for Foreign Medical Graduates - en el desarrollo de proyectos de otros países - Italia-.

Otro ámbito de formación y de evaluación de personal sanitario es el de personal de atención sanitaria inmediata y se ha publicado el Decreto 225/1996, de 12 de junio, que regula su formación y prevé la evaluación por el IES del personal de las ambulancias. Respecto a estos profesionales se ha editado una Guía de Formación y se ha previsto la realización de exámenes de evaluación.

\subsubsection{RELACIONES EXTERNAS}

El IES tiene suscritos convenios de colaboración con la Oficina Regional Europea de la Organización Mundial de la Salud de la cual es centro colaborador. El IES continua colaborando en el desarrollo de las tecnologías para la evaluación de la competencia profesional de los médicos con el National Board of Medical Examiners -NBME - y el Educational Comision for Foreign Medical Graduates -ECFMG_. A parte de las relaciones externas ya citadas, el IES continua manteniendo contactos con la Asociation for Medical Education in Europe -AMEE-.

El IES, también, tiene firmado convenios con la Facultad de Medicina de la Universidad de Calgary —Alberta, Canadá_ y a través del Departament de Sanitat y con el Centre d'Evaluation des Sciences de la Santé —CESSUL- de l'Université du Laval —Québec-.

También se destaca las relaciones internacionales del Programa de Investigación y Monitorización de las Enfermedades Crónicas para el Proyecto MONICA.

\subsection{EMPRESAS PÚBLICAS DEL SCS}

El Servei Català de la Salut, para gestionar y administrar los servicios y las prestaciones del sistema sanitario público puede emplear diversas formas de gestión — directa, indirecta o compartida-, que le permiten avanzar en la incorporación de mecanismos de gestión empresarial adecuados al carácter prestacional de la Administración Sanitaria, y avanzar también en la puesta en funcionamiento de nuevas estructuras organizativas que garanticen la calidad de 
los servicios e introduzcan a la vez una mayor eficacia y eficiencia en el sistema sanitario catalán a través de la competitividad entre los proveedores. De acuerdo con la LOSC y la Ley del Estatuto de la Empresa Pública Catalana, se han creado nueve empresas públicas adscritas al SCS o bien participadas por éste. Tres de estas nueve empresas que se mencionan se crean a raíz de los traspasos en materia sanitaria de los servicios y los recursos de las diputaciones de Girona, Lleida y Tarragona, que el Parlamento de Catalunya ratificó en junio de 1992 —Institut d'Assistència Sanitària, Gestió de Serveis Sanitaris y Gestió i Prestació de Serveis de Salut-, respectivamente. Sus funciones, establecidas en los estatutos, $\operatorname{son}^{11}$ :

1. Prestar servicios de atención sanitaria y de salud mental, y también de promoción de la salud, la prevención de la enfermedad y la atención sociosanitaria a la población.

2. Llevar a cabo programas institucionales en materia de protección de la salud, prevención de la enfermedad, asistencia sanitaria y sociosanitaria y rehabilitación.

3. Desarrollar programas de investigación en el ámbito de la salud y colaborar con las universidades y el resto de las instituciones competentes en materia de docencia e investigación.

Estas son las nueve empresas adscritas al Servei Català de la Salut:

1. Institut de Diagnòstic per la Imatge.

2. Energètica d'Instal.lacions Sanitàries, SA.

3. Gestió de Serveis Sanitaris.

4. Gestió i Prestació de Serveis de Salut.

5. Institut d'Assistència Sanitària.

6. Sistema d'Emergències Mèdiques, SA.

7. Agència d'Avaluació de Tecnologia Mèdica.

8. Institut Català d'Oncologia.

9. Serveis Sanitaris de Referència—Centre de Transfusió i Banc de Teixits.

\subsubsection{INSTITUT DE DIAGNÒSTIC PER LA IMATGE}

Los objetivos prioritarios del Institut de Diagnòstic per la Imatge - IDIson la gestión, la administración y la ejecución de los servicios de diagnóstico

11 Siguiendo la Memòria d'activitat 1996 del Servei Català de la Salut [1997, p. 129]. 
de alta tecnología mediante la utilización de diversas técnicas de diagnóstico por la imagen. Sus funciones principales son las siguientes ${ }^{12}$ :

1. Prestar asistencia a los ciudadanos por medio de la utilización de técnicas de diagnóstico por la imagen.

2. Desarrollar programas de investigación, colaborar con la universidad y otras instituciones competentes en materia de docencia y de investigación.

3. Colaborar con las Administraciones Públicas con competencia sanitaria en la elaboración de estudios relacionados con la tecnología por la imagen.

4. Llevar a cabo todas las actuaciones que sean necesarias para el cumplimento de sus objetivos y de sus funciones.

El Institut de Diagnòstic per la Imatge fue la primera empresa pública del SCS y fue creada por medio del Acuerdo del Gobierno de la Generalitat de Catalunya de 21 de diciembre de 1990 bajo la denominación de «Centre de Ressonància Magnètica de la Ciutat Sanitària Vall d'Hebron», posteriormente se autoriza el SCS a modificar la denominación y sus estatutos y, así, pasa a denominarse Institut de Diagnòstic per la Imatge.

Con la ampliación del ámbito de actuación del IDI fuera del área metropolitana de Barcelona, con objetivo de acercar la alta tecnología diagnóstica al resto del territorio catalán, de acuerdo con los criterios de equidad y accesibilidad que contiene la LOSC, se ha puesto en funcionamiento la Unidad de Resonancia Magnética del Hospital Arnau de Vilanova de Lleida y la Unidad de Resonancia Magnética del Hospital Joan XXIII de Tarragona.

12 Siguiendo parcialmente la Memòria 1996 del Institut de Diagnòstic per la Imatge [1997, p. 7]. 
FIGURA 6.13. Organigrama del IDI.

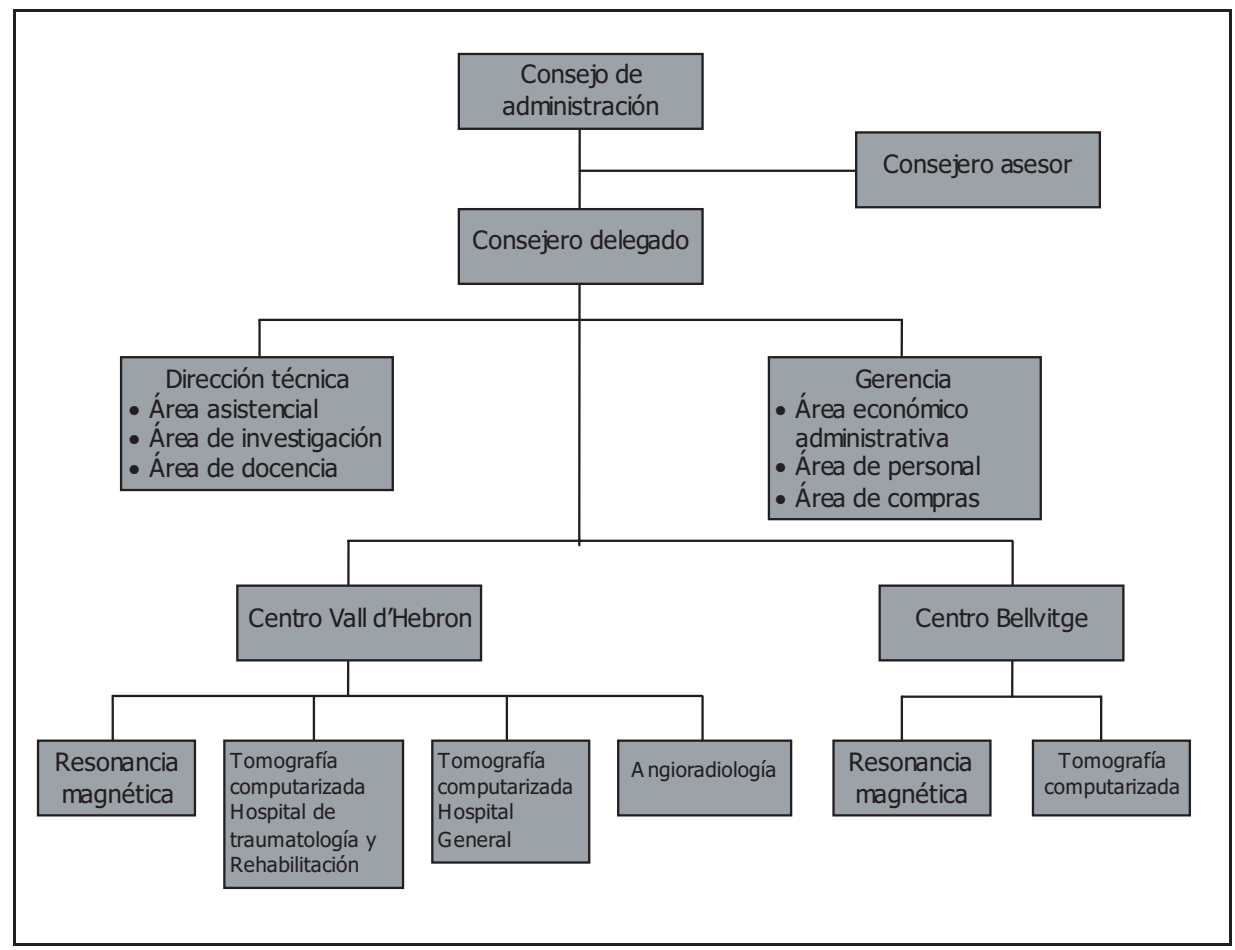

(C) Memòria 1996 del Institut de Diagnòstic per la Imatge [1997, p. 10].

\subsubsection{ACTIVIDAD ASISTENCIAL DEL IDI}

\section{Resonancia magnética}

En 1996 las unidades de resonancia magnética — RM — pasaron por la renovación de los aparatos de los centros IDI Bellvitge e IDI Vall d'Hebron. Esta renovación permitió al IDI seguir trabajando con tecnología punta en un campo en constante y rápida evolución tecnológica. También representó un esfuerzo por parte de los dos centros, que han tenido que coordinar sus acciones para que el tiempo de paro forzoso no repercutiera excesivamente sobre la presión asistencial — lo cual se ha conseguido aumentando la carga de trabajo, especialmente los sábados-. 
FIGURA 6.14. Número total de exploraciones por año.

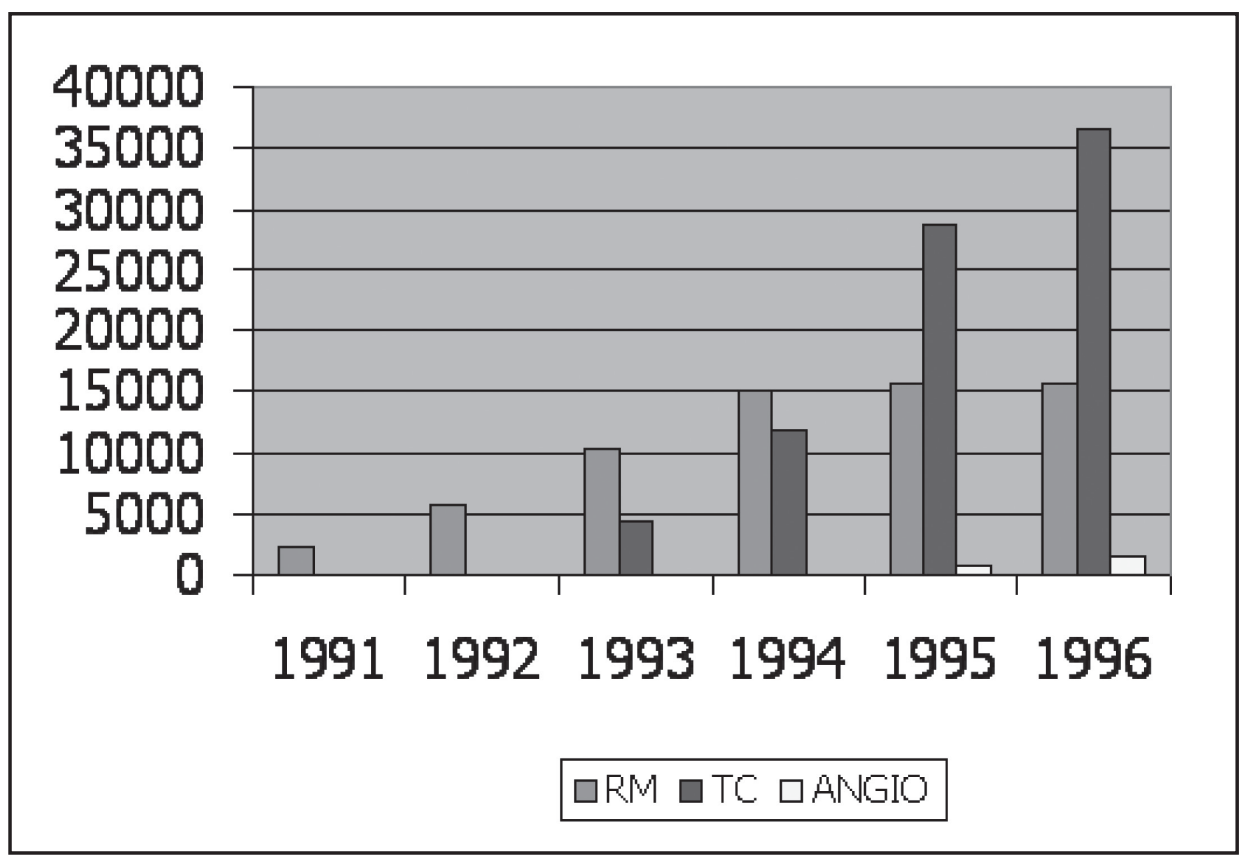

(C) Memòria 1996 del Institut de Diagnòstic per la Imatge [1997, p. 18].

En la figura 6.14 referente al número total de exploraciones se puede observar, a pesar de que la máquina del Centro Bellvitge no trabajó entre el 12 de agosto y el 30 de septiembre de 1996 y de que la máquina del Centro Vall d'Hebron estuvo parada a partir del 2 de diciembre de 1996, que el número total de exploraciones es equivalente al que se alcanzó en 1995 - 15.619 exploraciones en 1996 frente a 15.587 exploraciones en 1995-. La figura 6.14 muestra una clara saturación de las pruebas de RM en los tres últimos años. Con el cambio de máquinas se espera sobrepasar este límite en los próximos años.

\section{Tomografía computarizada}

Las cuatro unidades de tomografía computarizada - TC, tres del Centro Vall d'Hebron y una del Centro Bellvitge- han consolidado su actividad asistencial por encima de la media de exploraciones previstas. Si en el año 1995 el número total de exploraciones fue de 28.788 , en el año 1996 se ha realizado un total de 36.502 , lo que representa un $26,8 \%$ más de actividad y da una media de 3.042 exploraciones mensuales. 


\section{Radiología vascular e intervencionista}

La radiología vascular e intervencionista amplió su horario de trabajo y actualmente ofrece dos turnos y urgencias. El número total de exploraciones en 1996 ha sido de 1.435, y en 1997 entró en el campo de la angiorradiología y neurorradiología intervencionistas. En la figura 6.14 se muestra el número total de exploraciones de RM, TC y angiorradiología correspondientes al ejercicio 1996 junto con los valores desde el inicio de la actividad. Con los datos obtenidos durante 1996 se ha podido evaluar el coste real de todas las pruebas de la cartera de servicios. Los datos de 1996, correspondientes a campos con suficiente experiencia, como RM y TC, han confirmado los ya obtenidos en el ejercicio anterior.

Fue muy importante la valoración en el campo de la angiorradiología, tanto diagnóstica como terapéutica, que de entrada representaba una gran incógnita por la falta de datos al respecto y la diversidad de pruebas existentes. Este banco de datos sobre el coste de las pruebas de RM, TC y angiorradiología se ha puesto a disposición de la Administración, y así se cumple una de las misiones fundacionales del Institut de Diagnòstic per la Imatge.

\section{Actividad de investigación}

La actividad de investigación ha seguido con las líneas de trabajo en imagen, en espectroscopia y en tratamiento de la imagen. Las fuentes de subvención han sido, algunas, externas. De esta manera se ha seguido con la participación de dos becas: una del Fondo de Investigación Sanitaria _FIS — de la Seguridad Social, y la otra de la Comisión Interministerial de Ciencia y Tecnología - CICYT-, hasta el año 1999. También se han conseguido cuatro subvenciones de laboratorios para investigación integrada en programas internacionales de RM en imagen. Por otra parte, el IDI concede una subvención para investigación que permite básicamente mantener a una becaria bioquímica dedicada a la investigación en espectroscopia y que se encarga de la espectroscopia en la Unidad de RM del Centro Bellvitge.

\section{Actividad docente}

Por las diferentes unidades del IDI han pasado los médicos residentes de los departamentos de radiología de los hospitales universitarios con los que los centros están relacionados. También se han formado médicos, especialmente de Íbero América, ya que los centros del IDI son centros de referencia de diversas casas comerciales para la formación de médicos especialistas. Asimismo, se han 
formado técnicos y diplomados en enfermería. El IDI se incorporó en 1996 al convenio entre la escuela de formación de técnicos especialistas en radiología de la Escola Bonanova y el Hospital Vall d'Hebron, de manera que personal del Centro Vall d'Hebron realiza tareas docentes en la Escola Bonanova y parte de las prácticas de los estudiantes tanto de RM como de TC se realizan en el Centro Vall d'Hebron. Un gran paso ha sido la equiparación de los médicos especialistas del Centro Vall d'Hebron con los médicos adjuntos del departamento de radiología a efectos de la vinculación a cargos docentes dentro de la Universidad Autónoma de Barcelona. A parte de las tareas habituales de participación en las sesiones clínicas y en los distintos cursos de doctorado, todos los años se realiza una nueva edición del Curso de Introducción Biofísica a la Resonancia Magnética. La participación en congresos internacionales y el gran número de publicaciones expresa plenamente que el IDI, como empresa pública, está logrando muy satisfactoriamente los objetivos asistenciales, de investigación y docencia para los que fue fundado.

\subsubsection{ENERGÈTICA D'INSTAL.LACIONS SANITÀRIES, SA}

El propósito social de la empresa Energètica d'Instal.lacions Sanitàries, SA —EISSA — es el establecimiento de programas, la ejecución de proyectos y la realización de actuaciones de todo tipo destinadas a mejorar la eficiencia energética de los centros sanitarios que configuran el Servei Català de la Salut.

EISSA es una empresa participada, al 50\%, por el SCS y por el Institut Català d'Energia, con naturaleza de sociedad mercantil con personalidad jurídica propia y su actuación se ajusta al derecho privado.

EISSA se rige por las disposiciones aplicables a las sociedades anónimas con las peculiaridades que se establecen en el capítulo 4 de la Ley 4/1985, de 29 de marzo, del estatuto de la empresa pública catalana. Los órganos de gobierno de EISSA son el Consejo de Administración y el consejero delegado, que tiene bajo su dirección un técnico y, para los temas económicos y jurídicos cuenta con soporte externo.

\subsubsection{GESTIÓ DE SERVEIS SANITARIS}

La empresa Gestió de Serveis Sanitaris gestiona diversos centros y servicios.

En el ámbito de la atención especializada gestiona el Hospital de Santa Maria de Lleida y realiza el soporte especializado en la atención primaria de salud. 
Concretamente, a partir de 1996 se ha incrementado el soporte en las siguientes especialidades ${ }^{13}$ :

- Oftalmología.

- Otorrinolaringología.

- Cirugía.

Con relación a la atención de la salud mental, gestiona la Unidad de Hospitalización de Agudos de Psiquiatría del Hospital de Santa Maria, con 48 camas, el Centro de Día de Salud Mental de Lleida y los centros de salud mental de Lleida, Tremp — que da cobertura a Vielha—, Tàrrega y Balaguer/ les Borges Blanques.

FIGURA 6.15. Organigrama de Gestió de Serveis Sanitaris.

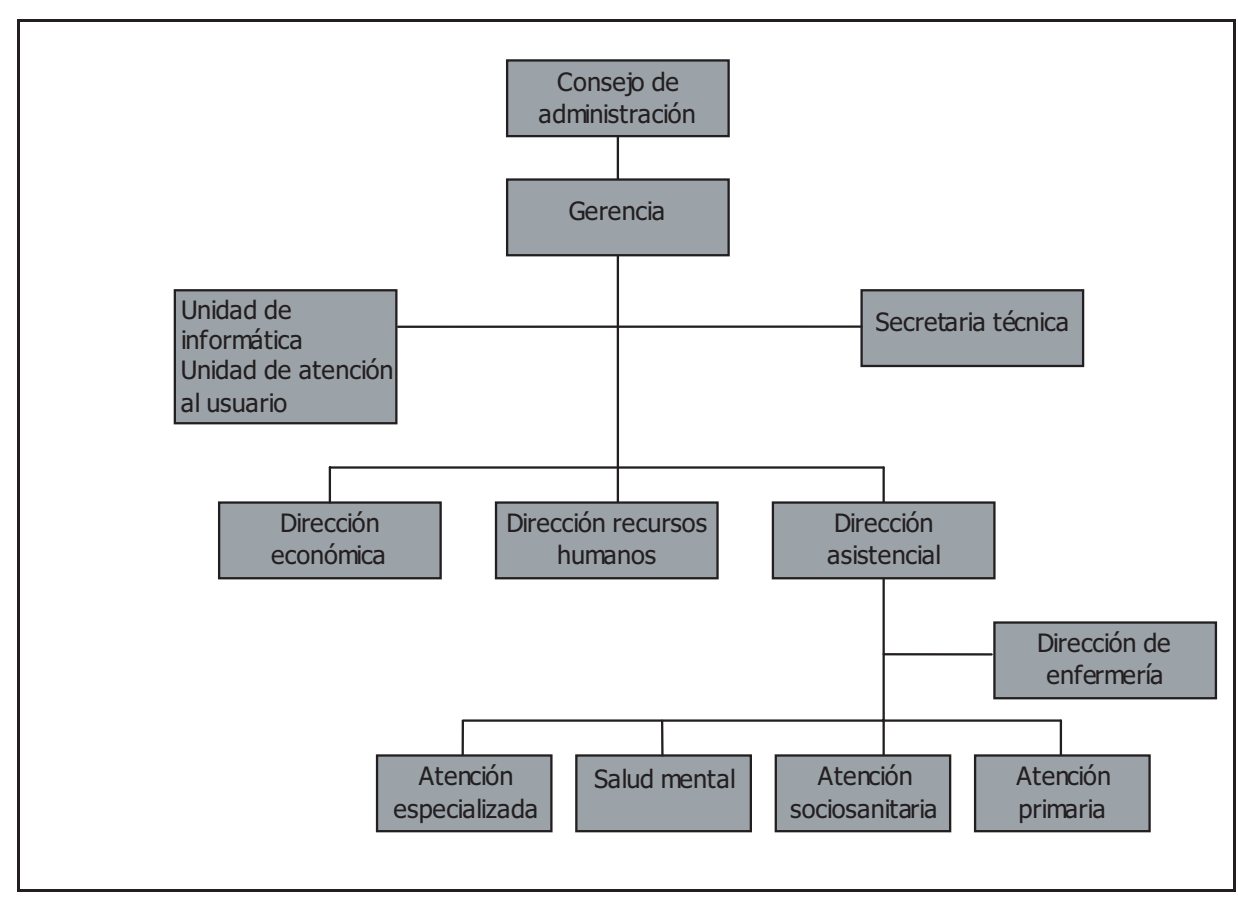

(C) Memòria d'activitat 1996 del Servei Català de la Salut [1997, p. 135].

En el mes de abril de 1996 se iniciaron las obras de remodelación del antiguo hospital psiquiátrico que concluyeron antes del verano de 1997 permitiendo

13 Siguiendo parcialmente la Memòria d'activitat 1996 del Servei Català de la Salut [1997, p. 135]. 
disponer de unas instalaciones completamente renovadas con 32 camas de agudos, 10 de subagudos, 4 de desintoxicación para drogodependencias y 10 plazas de hospital de día.

En el ámbito de las drogadicciones, Gestió de Serveis Sanitaris gestiona un centro de atención y seguimiento ambulatorio y una unidad de desintoxicación hospitalaria.

Con relación a la atención primaria de salud, en el mes de julio de 1996, se agregó en la empresa el Centro de Planificación Familiar Balàfia que hasta estos momentos era municipal.

\subsubsection{GESTIÓ I PRESTACIÓ DE SERVEIS DE SALUT}

La empresa Gestió i Prestació de Serveis de Salut gestiona las 57 camas del Hospital Mare de Déu de la Salut, que es un centro hospitalario dedicado a la atención sociosanitaria.

FIGURA 6.16. Organigrama de Gestió i Prestació de Serveis de Salut.

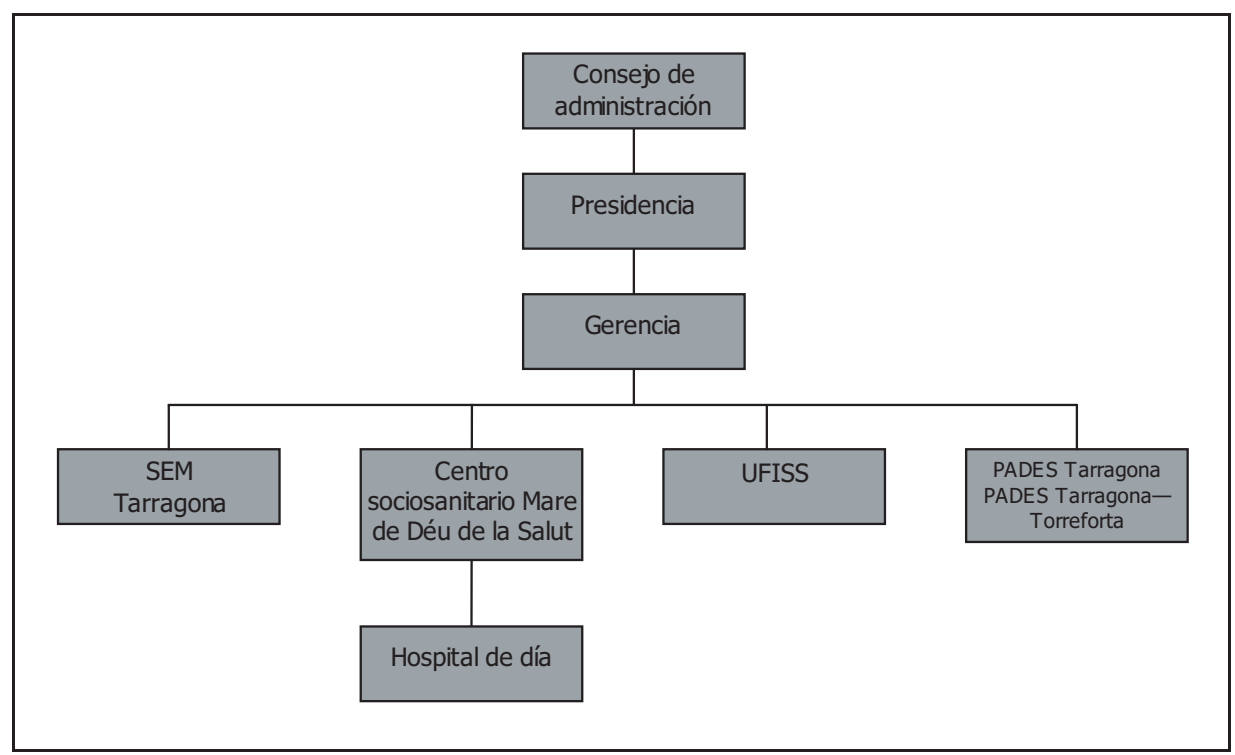

(C) Memòria d'activitat 1996 del Servei Català de la Salut [1997, p. 138].

De las 57 camas que tiene el centro, 39 son de larga estancia, 15 son de convalecencia y 3 de SIDA. También tiene 20 plazas de hospital de día.

En 1996 se firmó un convenio de colaboración entre la Asamblea Provincial de la Cruz Roja de Tarragona, dentro del ámbito de la prestación de servicios 
asistenciales primarios de emergencia, según el acuerdo contractual vigente entre la empresa pública Sistema d'Emergències Mèdiques, SA y la empresa Gestió i Prestació de Serveis de Salut.

\subsubsection{INSTITUT D'ASSISTÈNCIA SANITÀRIA}

En 1996 se configuró el Institut d'Assistència Sanitària - IAS - como empresa sanitaria que proporciona respuesta a las necesidades de salud de la población y se organiza eficazmente de acuerdo con la diversidad de ámbitos donde actúa. En este sentido, se aprobó el Documento marco para la reforma organizacional del IAS.

FIGURA 6.17. Organigrama del IAS.

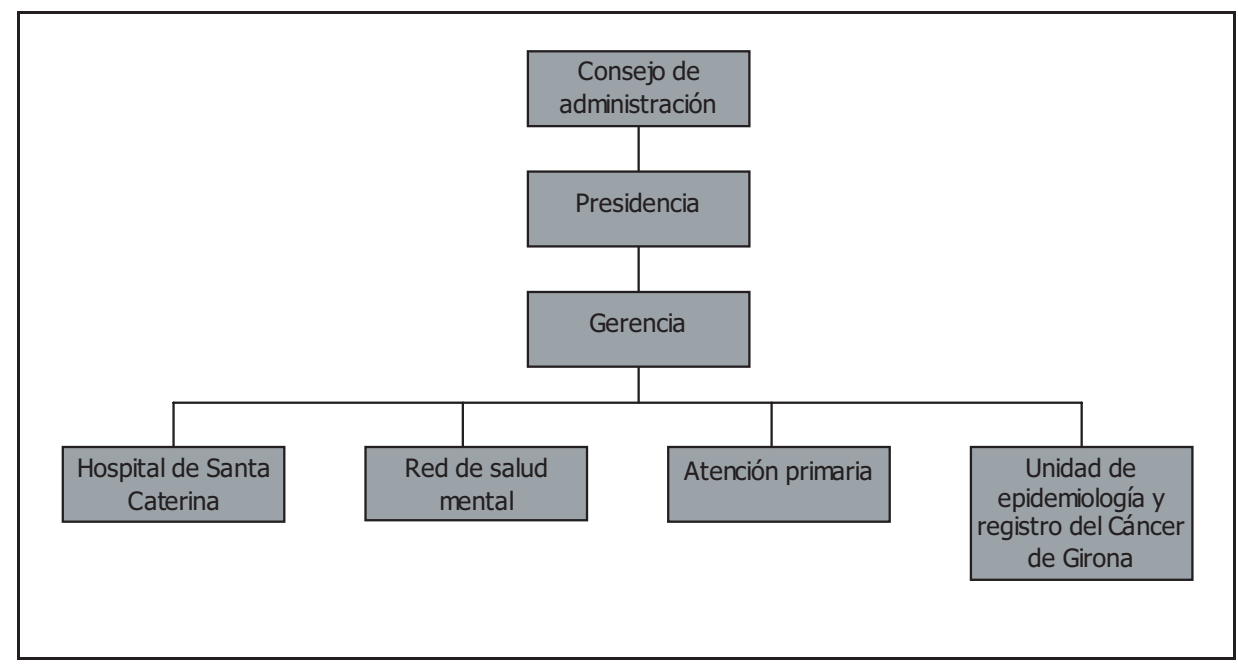

(C) Memòria d'activitat 1996 del Servei Català de la Salut [1997, p. 141].

El 1996 se colocó en funcionamiento la División de Atención Primaria y se han abierto el Centro de Salud Mental y Centro de Día del Baix Empordà y el Centro de Atención Primaria Hostalric. También se fusionaron los servicios que integran el parque hospitalario del IAS, formado por la unión del Hospital Santa Caterina y el Hospital Psiquiátrico de Salt.

En la empresa se potencia la vía de formación y docencia con la incorporación del MIR de psiquiatría, la definición del modelo de funcionamiento de la Comisión de Docencia y Formación y con la extensión de las actividades formativas al conjunto de la plantilla. 


\subsubsection{SISTEMA D'EMERGÈNCIES MÈDIQUES, SA}

La empresa pública Sistema d'Emergències Mèdiques, SA —SEMSA—, de acuerdo con sus estatutos, tiene como objetivo primordial la atención de las emergencias médicas por medio de la gestión y el control de los recursos personales, materiales y económicos disponibles para garantizar en todo momento la atención prehospitalaria, el transporte asistido y el ingreso en el centro adecuado de enfermos críticos.

FIGURA 6.18. Organigrama de SEMSA.

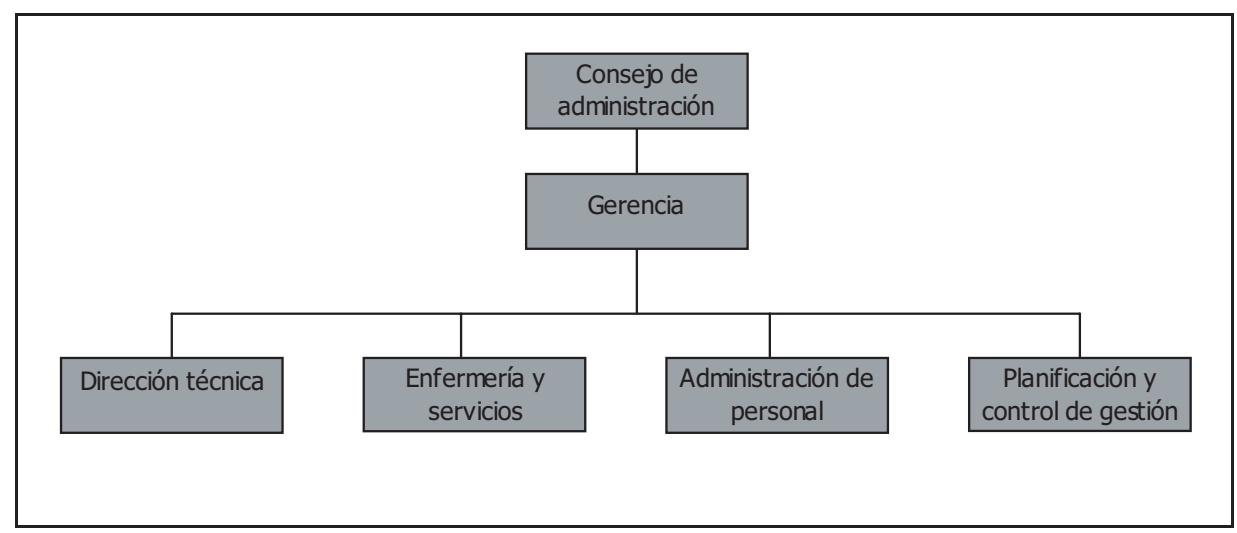

(C) Memòria d'activitat 1996 del Servei Català de la Salut [1997, p. 145].

Para el cumplimiento de su objetivo, funcionalmente la estructura de SEMSA es la siguiente ${ }^{14}$ :

1. Servicios centrales, donde se sitúan las direcciones operativas y administrativas de la sociedad.

2. El Centro coordinador, en funcionamiento las 24 horas del día, cuenta con un equipo de médicos y enfermeros en presencia física permanente que atiende y filtra personalmente las demandas de asistencia médica, coordina los recursos sanitarios disponibles, activa el medio móvil medicalizado que por sus características se adapta mejor a las necesidades asistenciales de cada uno de los casos que se presentan y, al mismo tiempo realiza el seguimiento a las 24 horas de todos los traslados interhospitalarios de enfermos críticos que se hayan realizado.

14 Siguiendo la Memòria 1996 de Sistema d’Emergències Mèdiques, SA [1997, p. 15]. 
3. Las Bases asistenciales, ubicadas mayoritariamente en centros hospitalarios de la red hospitalaria de utilización pública - XHUP_, están distribuidos en diferentes puntos estratégicos de la geografía de Catalunya, en función de la demanda asistencial de cada zona. Toda base asistencial está dotada de los siguientes recursos:

- Equipo asistencial: está formado por un médico, un enfermero y un auxiliar sanitario conductor/piloto.

- Medio móvil — terrestre o aéreo-: provisto de las características técnicas, la dotación y el equipamiento necesario para garantizar la aplicación de soporte vital básico y/o avanzado a los enfermos y accidentados en estado crítico.

En todos los servicios asistenciales de emergencia en que participa SEMSA a través de las diversas bases asistenciales, cuentan con el soporte médico del Centro Coordinador de Emergencias Médicas, equipado con un sistema de comunicaciones por radio y telefonía que permite establecer comunicación permanente con los diferentes alertadores del sistema - Bomberos, Protección Civil, etc.- , con los centros hospitalarios, con las bases asistenciales y con las unidades móviles.

El despliegue territorial de las bases asistenciales de qué dispone esta empresa pública, permite cubrir prácticamente el 100\% de las demandas de servicios de traslado interhospitalario de enfermos críticos, al mismo tiempo que da cobertura de servicios asistenciales primarios de emergencia a aproximadamente el 32\% de la población de Catalunya, exceptuando la ciudad de Barcelona - 27\% — que dispone de su propio servicio coordinador de urgencias médicas. 
TABLA 6.15. SEMSA, bases de actuación en las RS, 1996.

\begin{tabular}{|c|c|c|}
\hline Centro coordinador & Barcelona Ciutat & \\
\hline \multirow[t]{7}{*}{$\begin{array}{l}\text { Traslado } \\
\text { interhospitalario } \\
\text { de adultos }\end{array}$} & Lleida & $\begin{array}{l}\text { Hospital de Lleida Arnau de Vilanova } \\
\text { Sant Hospital de la Seu d'Urgell } \\
\text { - Hospital de Puigcerdà }\end{array}$ \\
\hline & Tarragona & $\begin{array}{l}\text { Hospital de Sant Joan, Reus } \\
\text { Hospital de Sant Pau i Santa Tecla [Servei } \\
\text { Comarcal d'Urgències del Vendrell] }\end{array}$ \\
\hline & Tortosa & Hospital de Tortosa Verge de la Cinta \\
\hline & Girona & $\begin{array}{l}\text { Hospital de Girona Doctor Josep Trueta } \\
\text { Hospital de Sant Jaume, olot } \\
\text { Hospital de Palamós }{ }^{1}\end{array}$ \\
\hline & Costa de Ponent & Ciutat Sanitària i Universitària de Bellvitge \\
\hline & $\begin{array}{l}\text { Barcelonès Nord i } \\
\text { Maresme }\end{array}$ & Hospital de Sant Jaume, Calella ${ }^{1}$ \\
\hline & Centre & $\begin{array}{l}\text { Consorci Hospitalari del Parc Taulí, } \\
\text { Sabadell } \\
\text { Helisuperfície/Hospital General de } \\
\text { Catalunya, } \\
\text { Sant Cugat del Vallès } 2\end{array}$ \\
\hline \multirow[t]{2}{*}{$\begin{array}{l}\text { Traslado } \\
\text { interhospitalario } \\
\text { pediátrico }\end{array}$} & Barcelona Ciutat & $\begin{array}{l}\text { Hospital Casa de Maternitat-Hospital Sant } \\
\text { Joan de Déu } \\
\text { Hospital Maternoinfantil Vall d'Hebron } \\
\text { Hospital de la Santa Creu i Sant Pau }\end{array}$ \\
\hline & Centre & $\begin{array}{l}\text { Helisuperfície/Hospital General de } \\
\text { Catalunya, Sant Cugat del Vallès² } \\
\text { Hospital General de Catalunya }\end{array}$ \\
\hline \multirow{4}{*}{$\begin{array}{l}\text { Servicios } \\
\text { asistenciales } \\
\text { primarios }\end{array}$} & Tarragona & Gestió i Prestació de Serveis de Salut \\
\hline & Costa de Ponent & Ciutat Sanitària i Universitària de Bellvitge \\
\hline & $\begin{array}{l}\text { Barcelonès Nord i } \\
\text { Maresme }\end{array}$ & $\begin{array}{l}\text { Hospital de Sant Jaume, Calella } \\
\text { Hospital de Badalona Germans Trias i Pujol }\end{array}$ \\
\hline & Centre & $\begin{array}{l}\text { Hospital General de Vic } \\
\text { Centre Hospitalari i Cardiològic de Manresa }\end{array}$ \\
\hline
\end{tabular}

(C) Memòria d'activitat 1996 del Servei Català de la Salut [1997, p. 145]. 
FIGURA 6.19. Ubicación de las bases asistenciales.

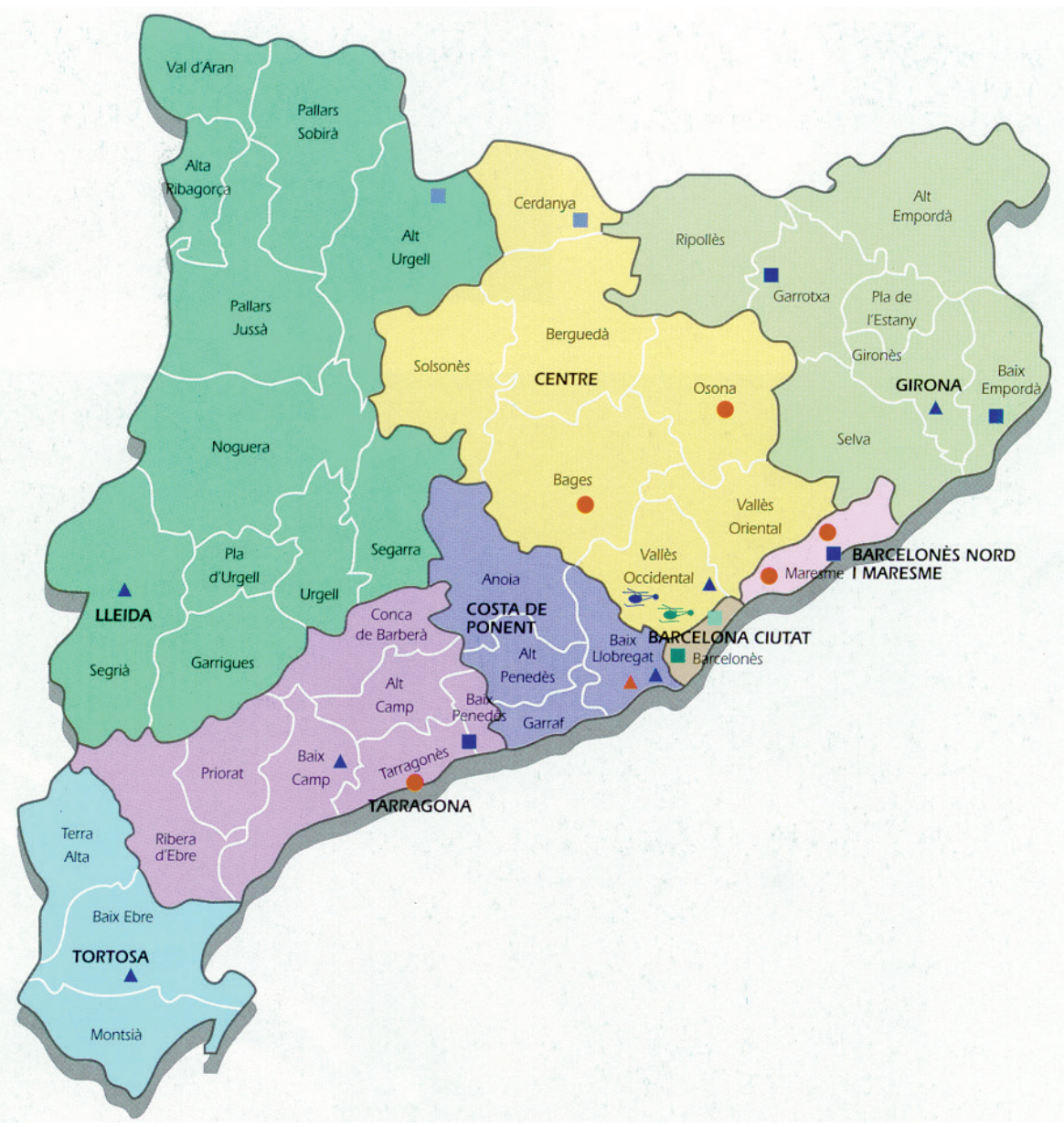

\section{Trasllat interhospitalari d'adults}

- Bases pròpies

- Bases col-laboradores

2- Mitjans aeris (helicòpter medicalitzat)

\section{Trasllat interhospitalari pediàtric}

- Bases col-laboradores

- Mitjans aeris (helicòpter medicalitzat)

\section{Serveis assistencials primaris d'emergència}

- Bases pròpies

- Bases de recursos compartits

(C) Memòria 1996 de Sistema d'Emergències Mèdiques, SA [1997, p. 17]. 


\subsubsection{ACTIVIDADES DEL SEMSA}

Las líneas de actuación que componen el servicio integral de emergencias médicas que la empresa pública Sistema d'Emergències Mèdiques, SA ha ofrecido a la población de Catalunya durante el ejercicio de 1996 han sido las siguientes ${ }^{15}$ :

\section{Coordinación de los recursos sanitarios disponibles}

Es un servicio que presta la empresa pública Sistema d'Emergències Mèdiques, SA de acuerdo con sus Estatutos, y comprende la coordinación de la información de los recursos hospitalarios y extrahospitalarios disponibles para garantizar en todo momento la asistencia de emergencia con la máxima celeridad posible.

Desde el inicio de la actividad del SEMSA como plan piloto en el año 1985 y hasta diciembre de 1996, constituido como empresa pública desde el abril de 1993 por el Acuerdo del Gobierno de la Generalitat de Catalunya del 14 de diciembre de 1992, se han coordinado más de 78.000 servicios de emergencia, se han prestado más de 55.000 servicios de traslado interhospitalario de adultos, más de 1.000 servicios de traslado interhospitalario pediátrico y más de 20.000 servicios primarios de emergencia.

\section{Traslado interhospitalario de enfermos críticos adultos}

Este servicio comprende el traslado medicalizado de aquellos enfermos críticos que, según criterio médico, requieren el traslado asistido hacia una unidad de curas intensivas o unidad de reanimación, desde un centro hospitalario a otro centro hospitalario con capacidad para asumir el nivel de gravedad y características del paciente.

\section{Traslado interbospitalario de neonatos y enfermos pediátricos}

Este servicio comprende el traslado medicalizado, adecuado para pacientes críticos tributarios de atención en una Unidad o Servicio de Neonatología o Pediatría, desde un centro hospitalario a otro.

Las demandas de servicios de traslado de enfermos críticos, sean adultos o pediátricos, son motivadas habitualmente por las siguientes circunstancias:

15 Siguiendo parcialmente la Memòria 1996 de Sistema d'Emergències Mèdiques, SA [1997, p. 20]. 
1. Pacientes que requieren tratamiento en una unidad de curas intensivas o unidad de neonatología o pediatría, y que proceden de un hospital con dotación y/o nivel no suficiente para la resolución del caso.

2. Pacientes procedentes de una unidad de curas intensivas que por motivos diagnósticos precisen de una tecnología no disponible en ese hospital.

3. Pacientes procedentes de un centro hospitalario con unidad de críticos, que no pueden ser asumidos por falta de camas.

\section{Servicios asistenciales primarios de emergencia}

Este servicio consiste en la asistencia a los accidentados y enfermos críticos en el mismo lugar donde se produce el accidente o la emergencia médica.

Comprende la valoración médica inicial, la estabilización del enfermo, la aplicación, si fuera necesario, de soporte vital básico y/o avanzado, y si el estado del enfermo lo requiere, la prescripción de traslado medicalizado al centro hospitalario más adecuado, teniendo en cuenta el tiempo de acceso, el nivel de gravedad y la patología de los enfermos, el nivel técnico y tecnológico del centro hospitalario receptor y la disponibilidad de camas.

\section{Asistencia protocolizada en accidentes de múltiples víctimas}

En todas aquellas zonas en que el Sistema d'Emergències Mèdiques, SA tiene una base asistencial, dicha empresa pública tiene asignada la asistencia a todos los accidentes de múltiples víctimas — AMV— que se produzcan.

Cuando se produce un suceso de estas dimensiones, el centro coordinador de SEMSA juega un papel importante dado que no únicamente activa la unidad asistencial que se encuentra en el lugar más cercano al siniestro, sino que activa si así lo considera necesario otras unidades ubicadas en bases más alejadas, a la vez que se comunica con todas las instituciones involucradas en estos acontecimientos y que son: Gerencia de Protección Civil de la Generalitat; Protección Civil del Estado; Bomberos de la Generalitat; Cruz Roja; Fuerzas y cuerpos de seguridad y Centros hospitalarios.

\section{Dispositivos sanitarios de riesgo previsible}

Estos dispositivos comprenden la colaboración y/o participación directa con otras entidades públicas o privadas en la coordinación de actuaciones sanitarias con el objetivo de dotar a la población de Catalunya de dispositivos sanitarios preventivos del riesgo en situaciones de grandes concentraciones humanas. 


\section{Actividad cientifica y de formación}

Los profesionales de SEMSA realizan múltiples actividades científicas y de formación continuada, a la vez que participan en congresos y sesiones monográficas relacionadas con la medicina de emergencia.

El objetivo del plan de formación es conseguir el mayor grado posible de polivalencia del personal que colabora con la empresa, a través de una formación integral que permite a los profesionales conocer globalmente toda la actividad que se realiza. Se intenta, por un lado, incentivar la participación y el compromiso de los profesionales en la actividad que prestan y, por otro lado, conseguir flexibilidad de adaptación y eficiencia en las tareas que desarrollan.

\subsubsection{CALIDAD ASISTENCIAL}

SEMSA trabaja para garantizar la prestación de servicios de medicina de emergencia de alta especificidad y calidad con el objetivo de contribuir en la disminución de la mortalidad y morbididad inherentes a las situaciones de emergencia.

Para conseguir una evaluación más directa y continuada de la actividad asistencial que realizan todos los equipos asistenciales que prestan servicios, SEMSA ha puesto en funcionamiento un proceso de implementación de un nuevo sistema de seguimiento y control de calidad más descentralizado, a través de una mayor implicación e interrelación de los responsables de las bases asistenciales.

El sistema que SEMSA tiene establecido con todos sus proveedores y colaboradores sanitarios exige una serie de estándares cualitativos que han de cumplirse en la prestación de los servicios y de los cuales se hace una valoración continuada realizada por los departamentos técnicos de esta empresa pública.

\subsubsection{AGÈNCIA D’AVALUACIÓ DE TECNOLOGIA MÈDICA}

\subsubsection{MARCO LEGAL}

La Agència d'Avaluació de Tecnologia Mèdica —AATM - es una empresa pública sin ánimo de lucro, adscrita al Servei Català de la Salut —SCS—, creada en mayo de 1994, por el Acuerdo del Gobierno de la Generalitat de Catalunya —DOGC núm. 1016, de 4.7.1994—. La AATM inició sus actividades como empresa el día 1 de enero de 1995. 
La misión de la AATM es dar soporte a la toma de decisiones para que la introducción, adopción, difusión y utilización de las tecnologías médicas - TMse hagan de acuerdo con los criterios de eficacia, seguridad, efectividad y eficiencia demostrados científicamente.

Por tecnologías médicas se entiende todo tipo de equipamientos, dispositivos, procedimientos preventivos, diagnósticos, terapéuticos y rehabilitadores, sistemas organizativos y de soporte utilizados en la atención sanitaria, excluyendo los medicamentos farmacéuticos, [Plan de salud de Catalunya 1996 — 1998, 1997, p. 46; Memòria 1996 Agència d'Avaluació de Tecnologia Mèdica, 1997, p. 79].

La AATM desarrolla sus actuaciones en el ámbito del Departament de Sanitat i Seguretat Social —DSSS—, del Servei Català de la Salut, de los centros sanitarios públicos y privados, además de otras administraciones y organizaciones sanitarias, entre diversos sectores.

\subsubsection{ESTRUCTURA ORGANIZATIVA}

La AATM se estructura fundamentalmente de esta manera, - Consejo de Administración, Comité Científico, Dirección y tres áreas funcionales, la de Análisis y Proyectos, la de Investigación y Formación y la de Información, Documentación y Comunicación—. La figura 6.20 muestra el organigrama de la AATM de forma esquematizada.

FIGURA 6.20. Organigrama de la AATM.

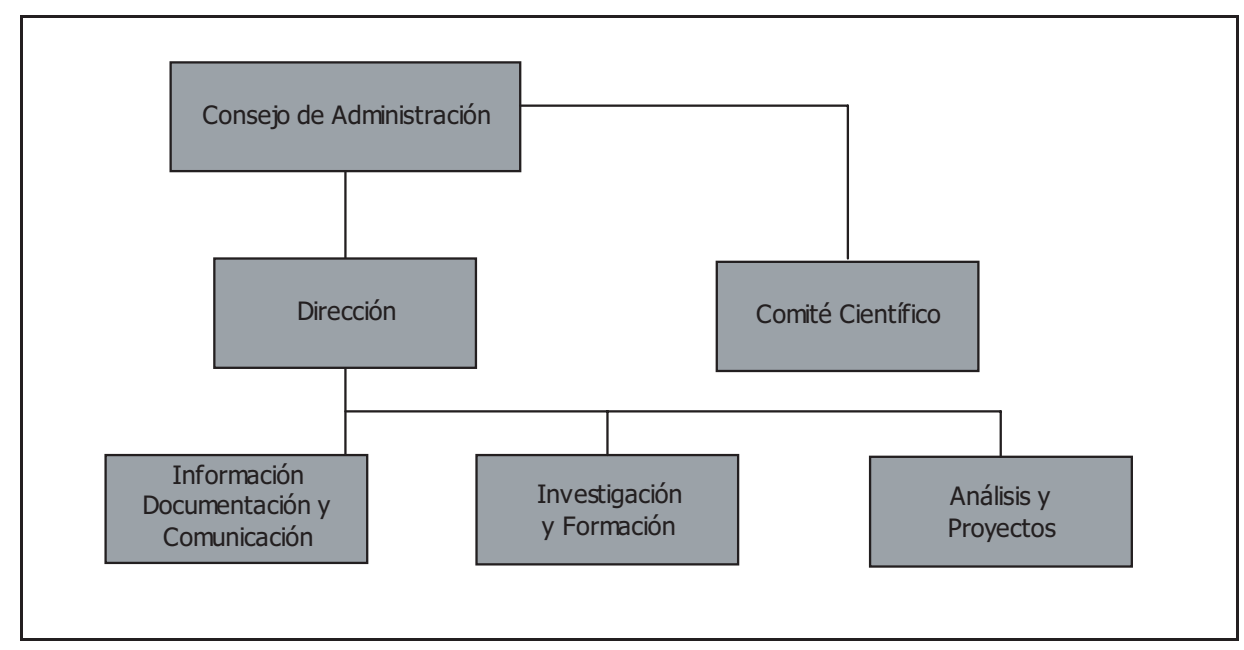

Memòria 1996 de la Agència d'Avaluació de Tecnologia Mèdica [1997, p. 80]. 


\subsubsection{MÉTODO DE TRABAJO}

El proceso global de evaluación de tecnologías médicas - ETM — consta de diferentes fases o etapas, en cada una de las cuales, como se verá a continuación, la participación y colaboración de los profesionales de la investigación y de la práctica clínica es considerada por la AATM como un elemento clave que contribuye a garantizar la calidad científica de los productos de la AATM, a la vez que hace extensivo el compromiso de mejorar la salud y la calidad de vida de la población a todos los actores del sistema.

La AATM combina metodologías diferentes en función, fundamentalmente, de la pregunta que debe responderse, del tipo de TM y/o el problema de salud a evaluar, de los objetivos concretos de cada estudio, del tipo de peticionario, $\mathrm{y}$ del tiempo y recursos disponibles. El proceso de evaluación consta de las siguientes fases ${ }^{16}$ :

1. Identificación y priorización de las TM que necesitan ser evaluadas. La identificación de las tecnologías, o binomios tecnologíacondición clínica, que necesitan evaluación, puede hacerse de forma preactiva, reactiva o mixta. Hay que considerar con detenimiento en este punto de inicio si la pregunta «política» puede traducirse adecuadamente en una «pregunta de investigación», lo cual implica enmarcarla desde el punto de vista de evidencia científica, de herramientas metodológicas y de medida del efecto.

En el seno de la AATM, la identificación de TM que necesitan evaluación parte de las prioridades contenidas en el Plan de Salud de Catalunya, de la valoración de las necesidades de evaluación efectuada por los miembros de su Comité Científico, y de la selección que hace el propio equipo, con criterios de disponibilidad de tiempo y recursos, siendo la propuesta de proyectos a realizar priorizada y aprobada por el Consejo de Administración, que tiene en cuenta a la vez criterios de incertidumbre hacia la TM, de impacto epidemiológico y/o económico, de variación en la práctica clínica, y de expectativa social, entre otros.

El proceso también puede iniciarse a petición del Departamento de Sanidad, del Servicio Catalán de la Salud, de centros sanitarios

16 Siguiendo parcialmente Memòria 1996 de la Agència d'Avaluació de Tecnologia Mèdica [1997, p. 20]. 
públicos o privados, de las entidades de seguros, y de la industria biomédica. Es así como los diferentes profesionales hacen llegar sus propuestas de evaluación a través de los centros o sociedades científicas que consultan la AATM.

2. Revisión y/o producción de evidencia científica. Toda posible área de conocimiento relacionada con la tecnología o condición clínica susceptible de evaluación debe ser sistemáticamente revisada, -investigación clínica, datos epidemiológicos, entre otros-. Una vez obtenida la información acerca de la eficacia, seguridad y efectividad, hay que clasificarla según la calidad de la evidencia aplicando la escala de calidad. Cuando se revisa la evidencia científica disponible puede resultar que no haya suficiente o que esta no sea concluyente. En este caso, es necesario estimular la producción de datos primarios en el entorno. Los profesionales sanitarios catalanes también participan en la producción de evidencia científica mediante el desarrollo de proyectos de investigación específicos.

3. Análisis del contexto —aspectos legales, éticos, de organización, sociológicos, económicos-. Antes de emitir las conclusiones y/o recomendaciones, es necesario contextualizar la información obtenida, y enmarcarla en el ámbito del sistema sanitario catalán y/o del peticionario concreto en cada caso.

4. Recomendaciones para la toma de decisiones. Las conclusiones obtenidas al aplicar el proceso de evaluación se especifican en recomendaciones o en el diseño de estrategias dirigidas a los responsables de tomar decisiones en el marco del sistema sanitario. Es importante recordar en este punto que antes de proceder a emitir y diseminar las recomendaciones, los informes de evaluación de la Agencia se somete tanto a un proceso de revisión interna como de revisión externa, contando una vez más con la participación de los profesionales sanitarios catalanes, procedentes del ámbito de la práctica clínica, que aportan así su experiencia y conocimientos, y forman parte activa del proceso multidisciplinario e integrador de la evaluación.

5. Diseminación y, cuando sea posible, implementación de los resultados de la ETM. El producto de la ETM se difunde de forma activa, utilizando un formato en cada caso, un lenguaje y un medio de comunicación adecuado a las características del grupo destinatario 
principal de la información. El siguiente paso es la implementación, entendida como una actividad que comporta el despliegue y la instauración de las actuaciones y medios necesarios para aplicar los resultados que se ha obtenido del proceso de ETM, a través del diseño de estrategias específicas con un presupuesto concreto.

6. Análisis del impacto de la ETM. Finalmente, el análisis del impacto de los diferentes productos de la ETM en el proceso de toma de decisiones constituye una fase muy importante del proceso global de evaluación, que permite medir la capacidad de inducir cambios positivos en las actitudes, en la práctica clínica y en la mejoría de resultados. Tanto desde el punto de vista de salud como de calidad de vida. Ello permite realizar una evaluación continuada del eventual éxito de las estrategias de diseminación e implementación y, lo que es más importante, monitorear la utilidad de la ETM para los responsables de la toma de decisiones.

\subsubsection{ACTIVIDADES DE LA AATM}

La AATM realiza diversos tipos de actuación, tanto por demanda externa como por iniciativa propia.

\section{Por demanda externa}

Hasta la fecha, la Agencia tiene tipificados 4 productos, - Informe de evaluación, Consulta específica, Consulta rápida, y Servicio de respuesta inmediata-.

1. Informe de evaluación. Se trata del documento resultante de un proceso de evaluación donde se han considerado todos los parámetros y variables que caracterizan este proceso -identificación de la prioridad a evaluar, producción y/o análisis y síntesis de datos científicos, económicos, de infraestructura, de aspectos éticos y legales - dentro del contexto sanitario analizado y orientado a las necesidades del peticionario concreto.

2. Consulta específica. Se trata de un documento de menor envergadura y complejidad, que se realiza y entrega en un período de tiempo menor al correspondiente a la realización de un informe de evaluación propiamente dicho. El contenido de información básico de este documento sirve al peticionario para tomar una decisión 
científicamente fundamentada y en el plazo en el que necesita hacerlo.

3. Consulta rápida. Se trata de informes cortos que dan respuesta de manera rápida y precisa a consultas muy concretas, mayoritariamente sobre normativa técnica y disposiciones legales relativas a tecnologías médicas y también sobre la distribución y difusión de determinadas tecnologías.

4. Servicio de respuesta inmediata —SRI—. Este servicio da respuesta a consultas puntuales, bien personalmente, por teléfono o mediante una breve carta.

La AATM realiza además de estos productos y servicios, a petición tanto del Departament de Sanitat i Seguretat Social como del Servei Català de la Salut, informes técnicos relativos a los procesos de autorización administrativa, consulta previa y contratación de servicios e inversión en equipamientos. Se trata de documentos técnicos destinados a informar acerca del proceso que siguen los expedientes de autorización administrativa y/o consulta previa de los establecimientos sanitarios y sociosanitarios, o bien la contratación de servicios e inversión en equipamientos. La demanda externa puede llegar de los ámbitos siguientes — se cita en primer lugar el de las instituciones públicas-:

- Departamento de Sanidad y Seguridad Social.

- Servei Catalán de la Salut — servicios centrales y servicios periféricos -

- Instituto Catalán de la Salud -ICS—: servicios centrales, división hospitalaria y de atención primaria.

- Instituto de Estudios de la Salud -IES-.

- Centros hospitalarios del ICS.

- Departamentos de la Generalitat de Catalunya.

- Centros hospitalarios no del ICS - XHUP y no XHUP-.

- Consorcios hospitalarios.

- Ciudadano particular.

- Entidades de seguro libre de asistencia médico—farmacéutica.

- Industria del sector sanitario —SS—, laboratorios, fabricantes, distribuidores.

- Sociedades científicas.

- Centros extrahospitalarios.

- Colegios oficiales.

- Fundaciones.

- Centros de Formación. 
- Consultorios.

- Otros, —incluye las agencias de evaluación, ministerios de sanidad y otras instituciones del sector sanitario de fuera de Catalunya-.

\section{Por iniciativa propia}

La Agencia realiza informes técnicos de evaluación que hacen referencia, principalmente, a incertidumbres relativas a técnicas y procedimientos médicos y quirúrgicos $\mathrm{y} / \mathrm{o}$ condiciones clínicas específicas, que pueden haber sido identificadas y propuestas por su comité científico o bien estar relacionadas con las prioridades contenidas en el Pla de Salut de Catalunya. También pueden haber sido identificadas por el propio equipo de la AATM, considerando el contexto sanitario internacional. Estos informes técnicos de evaluación se editan en catalán y castellano, y contienen un resumen en inglés. La AATM edita también un boletín informativo en catalán, castellano e inglés. Ambos tipos de documentación se distribuyen de forma gratuita, al igual que los ejemplares adicionales solicitados.

\subsubsection{SERVEIS SANITARIS DE REFERÈNCIA—CENTRE DE TRANSFUSIÓ I BANC DE TEIXITS}

La empresa Serveis Sanitaris de Referència-Centre de Transfusió i Banc de Teixits —SSRCTBT - se creó por medio del Acuerdo del Gobierno de la Generalitat de Catalunya del 7 de marzo de 1995, pero no fue hasta el 11 de enero del 1996 cuando empezó sus actividades productivas en la promoción de la donación, el análisis de sangre y plasma sanguíneo y en el suministro de productos hemoderivados en el sector asignado por el DSSS.

Serveis Sanitaris de Referència_Centre de Transfusió i Banc de Teixits ha cubierto el servicio de transfusión de los Hospitales de la Vall d'Hebron. Igualmentehadesarrolladosuactividadenlas especialidades deimmunohematología y criobiología - banco de tejidos-.

Por otro lado, ha supervisado el cumplimento de la normativa sobre evaluación de la calidad de los bancos de sangre de su sector y ha colaborado en los estudios encomendados por el SCS en relación con la hemodonación y transfusión sanguínea. Con la actividad desarrollada en 1996, se ha llegado en la cifra de 11.066 unidades de sangre con la colaboración de las asociaciones de donantes y la red de delegados, lo que ha permitido un adecuado suministro a los 36 hospitales asignados. También se ha podido facilitar los excedentes de productos 
sanguíneos a otras comunidades autónomas que todavía son deficitarias. El plasma excedente — 8.520 litros, en 1996-, debidamente transformado por la industria en hemoderivados —albúmina, gammaglobulinas y factor VIII—, se ha distribuido en los hospitales del ICS.

Se destaca el carácter de referencia del área de immunohematología, que canaliza las determinaciones complejas, no solamente del sector asignado sino en otros lugares de España. Con relación al banco de tejidos, se destaca el aumento en la obtención de células progenitoras de sangre periférica, así como de las córneas procesadas. El área de transfusión, por medio de los diversos comités de transfusión de los hospitales, estableció diferentes protocolos de actuación que se han traducido en una reducción importante en el consumo de plasma fresco y plaquetas, así como en una estabilización del consumo de los concentrados de hematíe.

Los objetivos globales de SSRCTBT son los siguientes ${ }^{17}$ :

1. Obtención de las donaciones de sangre previstas de acuerdo con las previsiones establecidas —Equipo móvil y Banco fijo-.

2. Suministrar de productos derivados sanguíneos los centros del sector asignados y producción de plasma par la fabricación de productos farmacéuticos hemoderivados.

3. Proveer el sector asignado de los servicios necesarios en el ámbito de la medicina transfusional, immunohematología y transplante de tejidos.

4. Ampliación del ámbito de suministro de productos y servicios.

5. Colaboración en la mejoría de la calidad transfusional en el sector asignado para que se utilicen los productos estrictamente necesarios con la máxima calidad y controlar las potenciales reacciones desfavorables.

6. Adaptar los procedimientos y técnicas del Centro a las nuevas demandas generadas por los avances científicos.

7. Desarrollar nuevos proyectos de investigación consolidando la presencia del Centro en los diferentes ámbitos científicos.

8. Adecuar la organización administrativa basada en la contabilidad presupuestaria hacia un modelo empresarial utilizando los mecanismos de gestión de la empresa privada y la contabilidad financiera.

17 Siguiendo la Memòria de Serveis Sanitaris de Referència-Centre de Transfusió i Banc de Teixits 1996 [1997, p. 19]. 
FIGURA 6.21. Organigrama del SSRCTBT.

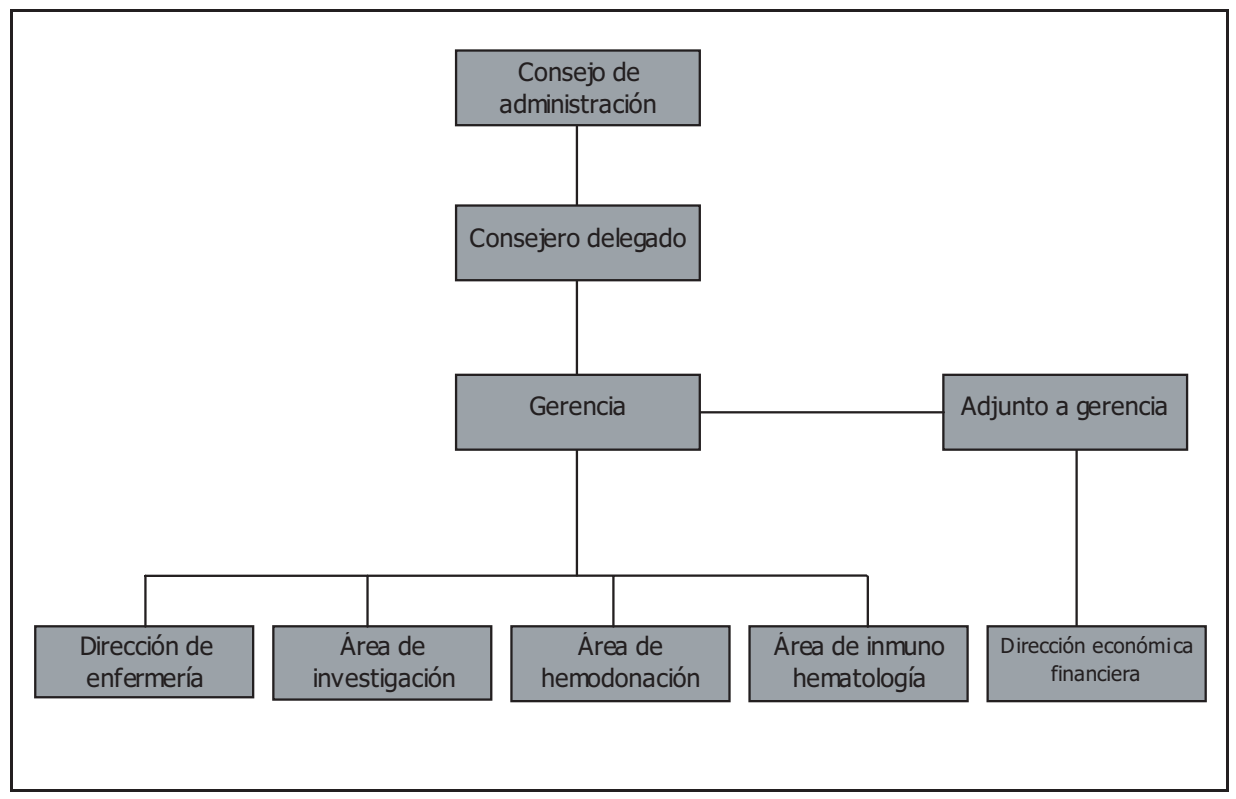

(C) Memòria de Serveis Sanitaris de Referència_Centre de Transfusió i Banc de Teixits 1996 [1997, p. 37].

\subsection{REGIÓ SANITÀRIA LLEIDA}

\subsubsection{DESCRIPCIÓN, TERRITORIO Y POBLACIÓN}

El ámbito territorial actual de la Regió Sanitària Lleida — RSL— se establece con la aplicación del Decreto 114/1994, de 3 de mayo, de delimitación de las regiones sanitarias y de los sectores sanitarios del Servei Català de la Salut —SCS — y la última orden de 28 de diciembre de 1995 de modificación de la ordenación territorial de diversas áreas básicas de salud.

La entrada en vigor de esta orden, con relación a la RSL, supone una pequeña disminución de la población del año 1996, frente a la población del año 1995. En concreto el ámbito territorial que comprende el municipio de Passanant, que hasta entonces formaba parte del Àrea Bàsica de Salut Tàrrega, pasa a formar parte del Àrea Bàsica de Salut Montblanc y, por tanto, de la Regió Sanitària Tarragona. Eso supone una disminución de la población en 194 habitantes, pertenecientes al municipio de Passanant. 
TABLA 6.16. Datos estructurales genéricos de la RSL.

\begin{tabular}{|lr|l|l|}
\hline Datos estructurales & Definición parámetro & Fuente \\
\hline Población & 338.337 & Núm. habitantes & Censo de población 1991 \\
\hline Extensión & $12.027,6$ & $\mathrm{Km}^{2}$ & $\begin{array}{l}\text { Anuario estadístico } \\
\text { Catalunya } 1991\end{array}$ \\
\hline Densidad de población & 29,4 & Habitantes/ Km² & $\begin{array}{l}\text { Anuario estadístico } \\
\text { Catalunya 1991 }\end{array}$ \\
\hline División territorial & 11 & Sectores sanitarios & Decreto 114/94 \\
\hline Renta per cápita & $1.228,40$ & $\begin{array}{l}\text { PTA por habitante } \\
\text { (renta disponible) }\end{array}$ & $\begin{array}{l}\text { Anuario estadístico } \\
\text { Catalunya 1992 }\end{array}$ \\
\hline Esperanza de vida & $75,4-81,5$ & Años para hombre/mujer & $\begin{array}{l}\text { Registro de mortalidad de } \\
\text { Catalunya. DSSS }\end{array}$ \\
\hline Tasa de mortalidad & 10,2 & $\begin{array}{l}\text { Defunciones por 1.000 } \\
\text { habitantes }\end{array}$ & $\begin{array}{l}\text { Registro de mortalidad de } \\
\text { Catalunya. DSSS }\end{array}$ \\
\hline Tasa de natalidad & 8,92 & $\begin{array}{l}\text { Nacimientos por 1.000 } \\
\text { habitantes }\end{array}$ & $\begin{array}{l}\text { Registro de mortalidad de } \\
\text { Catalunya. DSSS }\end{array}$ \\
\hline
\end{tabular}

(C) Memòria d'activitat 1996 de la Regió Sanitària Lleida [1997, p. 27].

La pirámide de edad de la población de la RSL es sensiblemente más envejecida que el resto de Catalunya [Pla de salut de Catalunya 1993 - 1995, p. 37]. La población de más de 65 años representa en la RSL el 18,2\% de los efectivos poblacionales frente al 14,3\% de Catalunya [Pla de salut de Catalunya 1996-1998, p. 37].

TABLA 6.17. Población de la RSL por edades.

\begin{tabular}{|l|c|c|c|}
\hline Años & Hombres & Mujeres & Total \\
\hline$<15$ & 29.325 & 27.641 & 56.966 \\
\hline $15-29$ & 38.868 & 37.100 & 75.968 \\
\hline $30-44$ & 34.212 & 32.686 & 66.898 \\
\hline $45-64$ & 37.711 & 39.073 & 76.784 \\
\hline$>65$ & 28.121 & 33.600 & 61.721 \\
\hline Total & $\mathbf{1 6 8 . 2 3 7}$ & $\mathbf{1 7 0 . 1 0 0}$ & $\mathbf{3 3 8 . 3 3 7}$ \\
\hline
\end{tabular}

(C) Memòria d'activitat 1996 de la Regió Sanitària Lleida [1997, p. 28].

El movimiento natural de la población en la RSL ha estado negativo [-1.207 personas con relación al padrón de 1986] con una tasa de decrecimiento negativo de $0,7 / 1.000 \mathrm{~h}$, todo y que, gracias a un saldo migratorio estimado positivo de 2.613 personas, se produce un incremento de efectivos poblacionales de 1.406 personas en relación con el padrón de 1986. 
En relación con la población catalana, la población de la RSL representa el $5,5 \%$ del total $[5,9 \%$ del total al padrón de 1986]. Hecho que contrasta notablemente con el 37,6\% que representa cuanto al territorio de la RSL en relación con el total de Catalunya.

\subsubsection{SECTORES SANITARIOS Y ABS}

La Ley 15/1990, de 9 de julio, de ordenación sanitaria de Catalunya —LOSCoptó con relación a la organización del Servei Català de la Salut —SCS— por una descentralización de los servicios que cuenta con una estructura organizativa desconcentrada, a través de las regiones sanitarias. Las regiones sanitarias se estructuran en Sectores Sanitarios —SS—, órganos desconcentrados, por medio de los cuales se desarrollan las actividades de prevención de la enfermedad, promoción de la salud, salud pública y la asistencia sanitaria en su nivel de atención primaria, así como las especialidades médicas de soporte y de referencia de ésta, coordinadamente con el nivel de la atención hospitalaria.

El ámbito territorial de los sectores se fija con el Decreto 114/1994, de 3 de mayo, de delimitación de las regiones sanitarias y de los sectores sanitarios del SCS, estableciendo 11 SS y una dirección operativa en la RSL. La delimitación de los SS se corresponde, como norma general, con las comarcas, de manera que la RSL está formada por los sectores:

- Les Garrigues.

- La Noguera.

- El Pla d’Urgell.

- La Segarra.

- El Segrià.

- L'Urgel.

- La Vall d'Aran.

- L'Alt Urgell.

- L'Alta Ribagorça.

- El Pallars Jussà.

- El Pallars Sobirà.

Durante el año 1995 se publicó la Ley 11/1995, de 29 de septiembre que modifica parcialmente la Ley 15/1990, de 9 de julio, de ordenación sanitaria de Catalunya.

Por un lado esta modificación adapta la LOSC a la nueva organización del SS, por otro, abre la posibilidad que los profesionales sanitarios gestionen, en régimen de 
asociación o cooperativa los centros, servicios o establecimientos sanitarios, con la finalidad de promover un mayor grado de implicación de los profesionales en el proceso de desarrollo, racionalización y optimización del sistema sanitario público.

FIGURA 6.22. Mapa de la RSL.

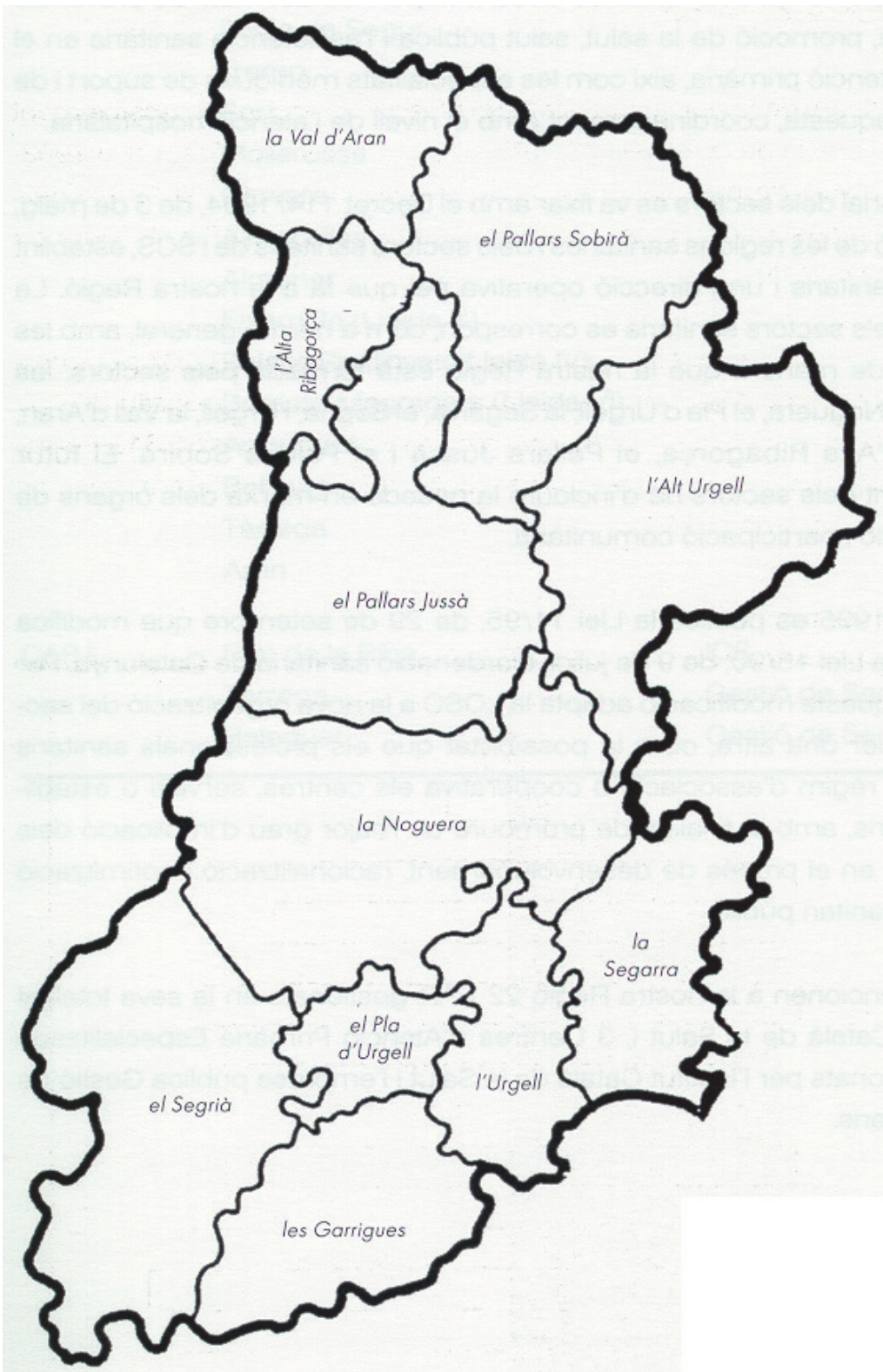

(C) Memòria d'activitat 1996 de la Regió Sanitària Lleida [1997, p. 29]. 
En 1996 funcionaron en la RSL 22 Equipos de Atención Primaria —EAP—, gestionados en su totalidad por el Institut Català de la Salut - ICS—, 3 Centros de Atención Primaria Especializada - CAP II-, gestionados por el ICS y la empresa pública Gestió de Serveis Sanitaris.

TABLA 6.18. Implantación de las ABS en la RSL.

\begin{tabular}{|l|l|l|}
\hline \multicolumn{2}{|l|}{ Centros } & \multicolumn{2}{c|}{ Entidad proveedora } \\
\hline CAP & $\begin{array}{l}\text { Oliana, Seu d'Urgell, El Pont de Suert, Les Borges Blanques, } \\
\text { La Granadella, Artesa de Segre, Balaguer, Ponts, Almacelles, } \\
\text { Pobla de Segur, Tremp, Sort, Mollerussa, Cervera, Almenar, } \\
\text { Eixample [Lleida III], Balàfia-Pardinyes [Lleida IV], Aran, } \\
\text { Bordeta-Magreners [Lleida VI], Agramunt, Bellpuig, Tàrrega. }\end{array}$ & ICS \\
\hline CAP II & Prat de la Riba. & ICS \\
\hline CAP II & Tàrrega, Balaguer. & Gestió de Serveis Sanitaris \\
\hline
\end{tabular}

Fuente: Elaboración propia a partir de la Memòria d’activitat 1996 de la Regió Sanitària Lleida [1997].

\subsubsection{ESTRUCTURA CORPORATIVA}

La LOSC establece que los órganos de dirección y gestión de las Regiones Sanitarias son el Consejo de Dirección, los gerentes y los órganos u organismos, los servicios y las unidades que se constituyen por reglamentación y, así mismo, que el Consejo de Salud es el órgano de participación en la RS — artículo 24 de la LOSC en anexo-.

Con relación a las unidades, el Comité Directivo del SCS acordó —en consecución de las funciones encomendadas a las regiones sanitarias- prever cinco ámbitos funcionales. En la RSL, ateniéndose a sus propias características, estos ámbitos funcionales se agruparon en las siguientes unidades:

- Planificación.

- Compra de servicios.

- Atención al cliente.

- Control de gestión.

- Dirección de sectores. 
FIGURA 6.23. Organigrama funcional de la RSL.

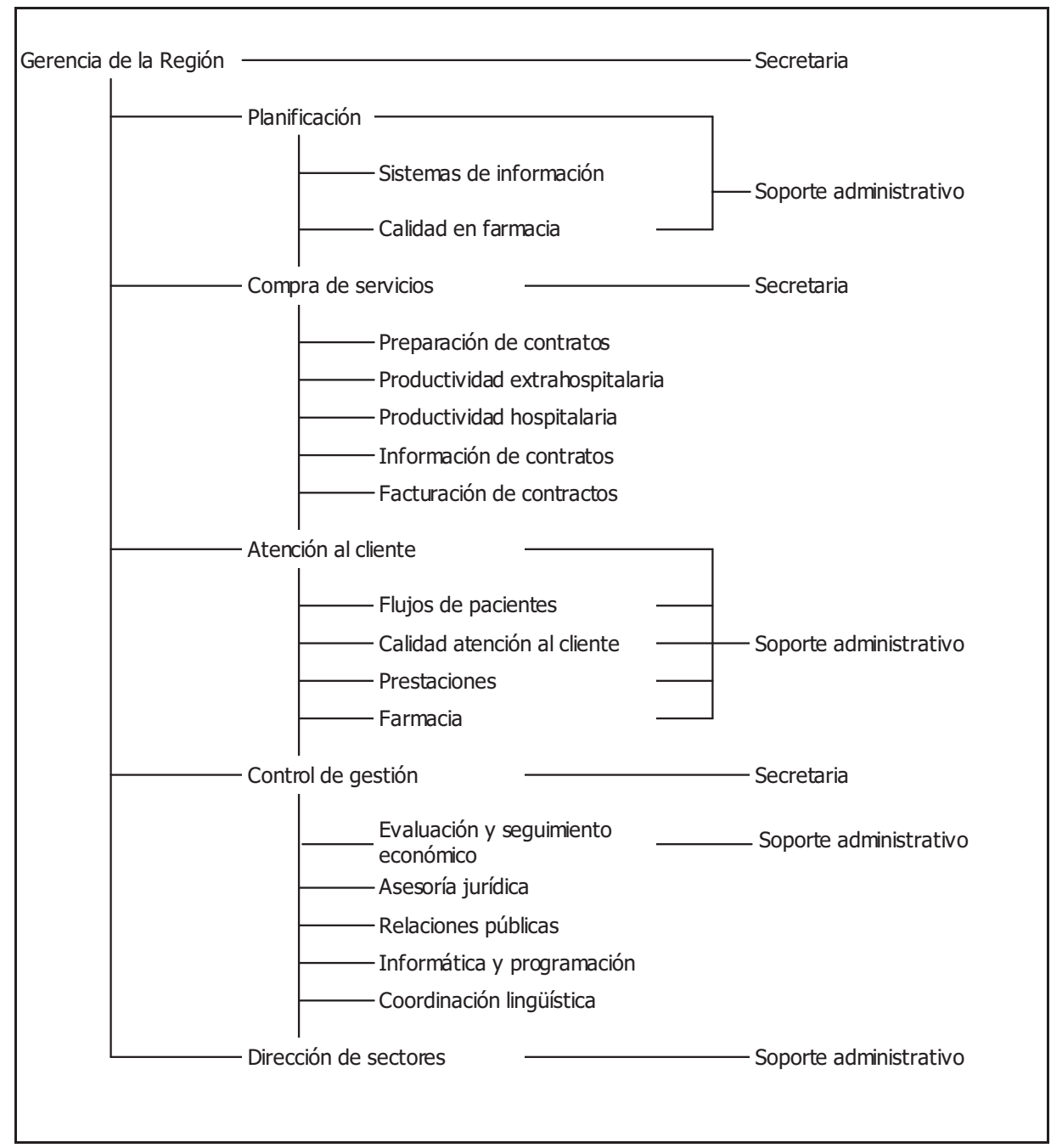

(C) Memòria d'activitat 1996 de la Regió Sanitària Lleida [1997, p. 45].

\subsection{REGIÓ SANITÀRIA TARRAGONA}

\subsubsection{DESCRIPCIÓN, TERRITORIO Y POBLACIÓN}

La Regió Sanitària Tarragona — RST - está situada en la parte sudoeste de Catalunya, con una extensión de 3.863,6 km². Actualmente comprende 7 comarcas, 4 de las cuales son interiores: 
- L'Alt Camp.

- La Conca de Barberà.

- El Priorat.

- La Ribera d'Ebre.

$\mathrm{Y}$ tres son litorales, con $85 \mathrm{Km}$ de franja costera:

- El Baix Camp.

- El Baix Penedès.

- El Tarragonès.

La población total de la RST es de 408.684 habitantes, según el censo de población de 1991, y está formada por dos tipos de población bien diferenciadas. Por un lado, las comarcas de la Conca de Barberà, del Priorat y de la Ribera d'Ebre tienen una población rural y muy envejecida, con una densidad baja y un decrecimiento de la población. Por otro lado, las comarcas costeras tienen una población joven, y durante los meses de verano experimentan un fuerte incremento de la población.

Entre 1986 y 1991 se produce en la RST un incremento de población del $4,35 \%$ superior al experimentado en Catalunya durante el mismo período - 1,3\%—. Este incremento no se produjo de forma homogénea en todo el territorio de la RST. Así, mientras que las comarcas de la Conca de Barberà, el Priorat y la Ribera d'Ebre perdieron población durante este período, el l'Alt Camp ha tenido un ligero crecimiento demográfico, que ha estado importante en el Baix Camp y en el Tarragonès y ha supuesto un fuerte crecimiento en el Baix Penedès.

Con una densidad media de población de 105,8 habitantes por $\mathrm{km}^{2}$, existe una gran divergencia entre las comarcas costeras —el Tarragonès: $461,8 \mathrm{~h} / \mathrm{km}^{2}-\mathrm{y}$ las del interior —el Priorat: 19,1 h/ $\mathrm{km}^{2}$ -

Como en el resto de Catalunya, la población se concentra en unos pocos núcleos urbanos —el 48,4\% de la población vive en las ciudades de Reus: 87.670 habitantes y Tarragona: 110.153 habitantes—. Las comarcas del Baix Camp y del Tarragonès concentran el 70,3\% de la población. La estructura de la población de la RST refleja el proceso de reducción de la natalidad y la tendencia al envejecimiento. El número de nacimientos registrados en el territorio de la RST en los últimos años se encuentra estabilizado en un número que oscila entorno a los 4.000 por año.

Por la proporción de población joven —-19,44\% menos de 14 años—, adulta — 66,14\% entre 15 y 64 años— y mayor —-14,42\% de más de 65 años—, la RST puede ser considerada como una región de tipología intermediaria como la de 
Girona, entre las regiones de Lleida, Tortosa y Barcelona — regiones envejecidasy las regiones de Costa de Ponent y Barcelonès Nord i Maresme —regiones jóvenes-.

En los próximos años se prevé que los cambios demográficos que se producirán serán más importantes con relación a su composición por edades que con su crecimiento. Estos cambios en la estructura de la población causarán las modificaciones más importantes en las demandas de prestaciones asociadas con la edad. Se prevén incrementos ligeros de la población, excepto en la Conca de Barberà, el Priorat y en la Ribera d'Ebre, y se incrementan los indicadores de envejecimiento de la población en todas las comarcas pero especialmente en estas tres anteriores, donde más de la cuarta parte de sus habitantes tendrán más de 65 años.

Con relación a la ocupación y según los datos del censo de población de 1991, la principal rama de actividad de la RST es la de servicios, con más del $50 \%$ de los ocupados, seguida de la industria, con poco más del 25\%, del sector primario - agricultura, ganadería y pesca- con el 12,27\% y de la construcción, con el $11,51 \%$.

\subsubsection{SECTORES SANITARIOSY ABS}

De acuerdo con el que establece la LOSC, la RST se ordena en subunidades territoriales integradas, conformadas cada una por un conjunto de áreas básicas de salud - ABS — , con una estructura desconcentrada de dirección, gestión y participación comunitaria. Estas subunidades se denominan sectores sanitarios —SS — y la RST está formada por los siguientes:

- L'Alt Camp.

- El Baix Penedès.

- La Conca de Barberà.

- El Tarragonès.

- El Baix Camp.

- El Priorat.

- La Ribera d'Ebre. 
TABLA 6.19. Ordenación territorial de la RST.

\begin{tabular}{|c|l|l|l|l|}
\hline \multicolumn{5}{|c|}{ Dirección operativa 2} \\
\hline SS & Alt Camp & Baix Panedès & Conca de Barberà & Tarragonès \\
\hline ABS & $\begin{array}{l}\text { Alt Camp Est, } \\
\text { Alt Camp Oest Valls } \\
\text { urbà. }\end{array}$ & $\begin{array}{l}\text { Baix Panedès } \\
\text { interior, } \\
\text { El Vendrell. }\end{array}$ & $\begin{array}{l}\text { Montblanc, } \\
\text { Sta. Coloma, } \\
\text { Queralt. }\end{array}$ & $\begin{array}{l}\text { Constatí, El Morell, Salou, } \\
\text { Tarragona-1, Tarragona-2, } \\
\text { Tarragona-3, Tarragona-4, } \\
\text { Tarragona-5, Tarragona-6, } \\
\text { Tarragona-7, Torredembarra, } \\
\text { Villa-seca. }\end{array}$ \\
\hline SS & Baix Camp & \multicolumn{3}{|c|}{ Dirección operativa 3 } \\
\hline ABS & $\begin{array}{l}\text { Les Borges del Camp, Cambrils, } \\
\text { Mont-roig del Camp, Reus-1, Reus-2, Reus-3, } \\
\text { Reus-4, Reus-5, Riudoms, } \\
\text { Vandellòs i L'Hospitalet de I'Infant. }\end{array}$ & $\begin{array}{l}\text { Carnudella del } \\
\text { Montsant } \\
\text { Falset. }\end{array}$ & $\begin{array}{l}\text { Flix, } \\
\text { Móra la Nova, } \\
\text { Móra d'Ebre. }\end{array}$ \\
\hline
\end{tabular}

Fuente: Elaboración propia a partir de la Memòria d'activitat 1996 de la Regió Sanitària Tarragona [1997].

Estos SS se estructuran en dos direcciones operativas: la 2, que incluye los sectores del Alt Camp, el Baix Penedès, la Conca de Barberà y el Tarragonès, $y$ la 3, que comprende los sectores del Baix Camp, el Priorat y la Ribera d'Ebre. En el momento de su despliegue, y para desarrollar las funciones que tienen encomendadas, se han definido dos unidades funcionales dependientes del director del sector: el ámbito del Análisis y Programación y el ámbito del Servicio al Cliente.

FIGURA 6.24. Mapa de la RST.

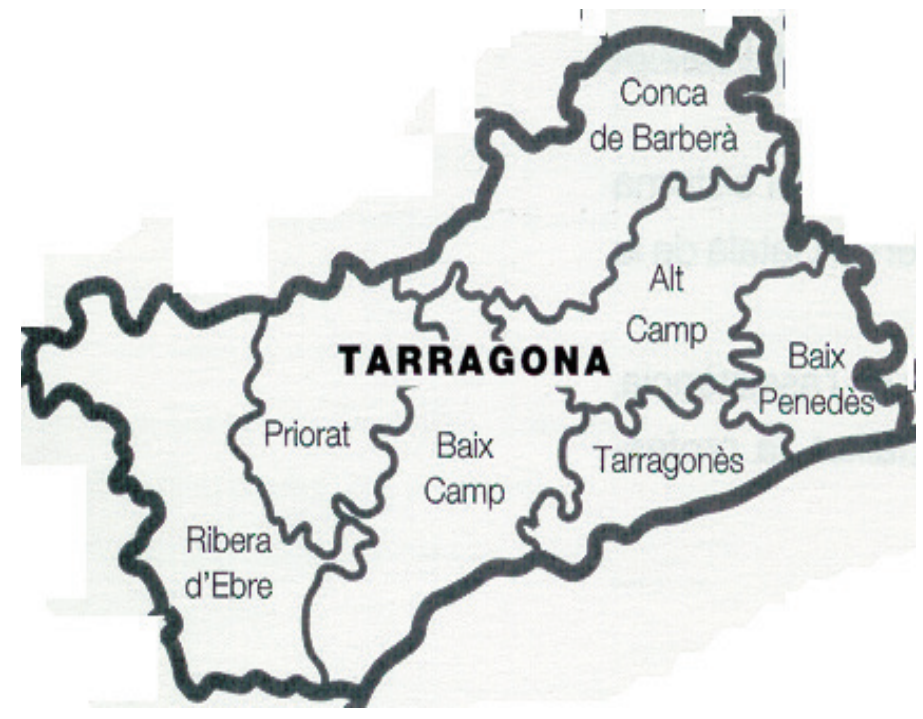

(C) Memòria d'activitat 1996 de la Regió Sanitària Tarragona [1997, p.16]. 


\subsubsection{ESTRUCTURA CORPORATIVA}

La RST tiene una estructura organizativa formada por cinco unidades funcionales:

- Planificación.

- Compra de Servicios.

- Atención al Cliente.

- Control de Gestió.

- Soporte Operativo.

Delante de cada una de estas unidades existe un responsable. Este equipo de gerencia incorpora también orientación en las diferentes líneas de servicios:

- Atención primaria de salud.

- Atención especializada y hospitalización de agudos.

- Atención sociosanitaria.

- Atención de la salud mental.

Desde la gerencia de la RST y con la coordinación de estos ámbitos por parte del responsable de la Unidad de Compra de Servicios, en cada línea de servicios existe un responsable, la misión principal del cual es ejercer de interlocutor de la RST en su ámbito concreto.

FIGURA 6.25. Organigrama de la RST.

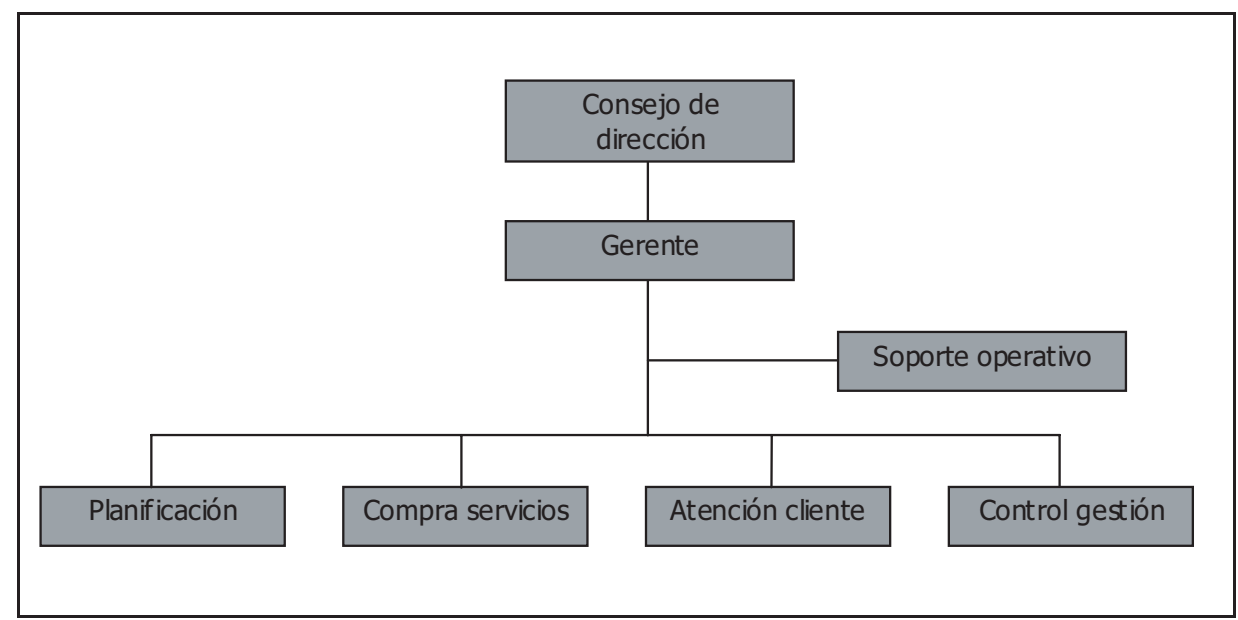

(C) Memòria d'activitat 1996 de la Regió Sanitària Tarragona [1997, p. 38]. 


\subsection{REGIÓ SANITÀRIA TORTOSA}

\subsubsection{DESCRIPCIÓN, TERRITORIO Y POBLACIÓN}

La Regió Sanitària Tortosa — RSTO_ comprende las comarcas más meridionales de Catalunya, según la delimitación de las regiones sanitarias establecida por el Decreto 114/1994, de 3 de mayo, y que son las siguientes:

- Baix Ebre.

- Montsià.

- Terra Alta.

La superficie total de la RSTO representa el 7,6\% del total de Catalunya, mientras que el total de población es solamente el 2,2\%. En cuanto a la densidad de población, la media de las tres comarcas está muy por debajo de la media de Catalunya que es 190 habitantes por $\mathrm{km}^{2}$. El Baix Ebre y el Montsià tienen una densidad de población similar, destaca por debajo la Terra Alta, con unos niveles más característicos de las comarcas del interior.

TABLA 6.20. Densidad de población de la RSTO.

\begin{tabular}{|l|r|r|r|r|}
\hline \multicolumn{2}{|c|}{ Baix Ebre } & Montsià & Terra Alta & Total \\
\hline Población & 64.645 & 54.307 & 12.945 & $\mathbf{1 3 1 . 8 9 7}$ \\
\hline Extensión en Km² & 987,9 & 708,7 & 740 & $\mathbf{2 . 4 3 6 , 6}$ \\
\hline Densidad de población & 65,4 & 76,6 & 17,5 & $\mathbf{5 4 , 1}$ \\
\hline
\end{tabular}

(C) Memòria d'activitat 1996 de la Regió Sanitària Tortosa [1997, p. 27].

En relación con la distribución municipal, de los 38 municipios que comprende el territorio de la RSTO, solo 4 sobrepasan los 10.000 habitantes y que agregan casi la mitad de la población —el 49,81\%—, y 3 que sobrepasan los 5.000 habitantes, con el 14,06\% de la población. El núcleo de población más frecuente es el que reúne entre 501 y 2.000 habitantes, con 17 municipios —el 44,74\%-.

Con relación a la evolución demográfica de la población, la tendencia del crecimiento natural es negativa para las tres comarcas en el período de 1986 a 1994. En cambio el saldo migratorio interior tiene valores positivos —en el caso de la Terra Alta esta tendencia positiva no se produce hasta 1992-. 
TABLA 6.21. Evolución demográfica de la RSTO.

\begin{tabular}{|l|r|r|r|r|}
\hline Tasa bruta de crecimiento natural & $\mathbf{1 9 9 1}$ & $\mathbf{1 9 9 2}$ & $\mathbf{1 9 9 3}$ & $\mathbf{1 9 9 4}$ \\
\hline Baix Ebre & $-2,52$ & $-2,18$ & $-2,13$ & $\mathbf{- 3 , 8 8}$ \\
\hline Montsià & $-0,97$ & $-1,43$ & $-2,38$ & $\mathbf{- 2 , 6 6}$ \\
\hline Terra Alta & $-5,80$ & $-3,34$ & $-9,04$ & $\mathbf{- 4 , 8 6}$ \\
\hline
\end{tabular}

(C) Memòria d'activitat 1996 de la Regió Sanitària Tortosa [1997, p. 28].

En cuanto a los movimientos migratorios exteriores, la mayoría de inmigrantes extranjeros, en el período 1992-1994, provienen de África —35,80\%—, seguidos de los provenientes de países europeos de la Unión Europea — 27,16\% y del resto de Europa — 23,25\% — [Memòria d'activitat 1996 de la Regió Sanitària Tortosa, 1997, p. 28].

El Institut d'Estadística de Catalunya estima que en 1994 la población de las comarcas de la RSTO se situaba entorno de 132.179 habitantes, lo que supone un mínimo crecimiento total del $0,21 \%$ de la población respecto al censo de 1991.

TABLA 6.22. Población por grupo, edad y sexo. RSTO.

\begin{tabular}{|l|r|r|r|}
\hline Grupo de edad & Hombres & Mujeres & Total \\
\hline $0-14$ & 11.931 & 10.987 & $\mathbf{2 2 . 9 1 8}$ \\
\hline $15-29$ & 14.615 & 13.740 & $\mathbf{2 8 . 3 5 5}$ \\
\hline $30-44$ & 12.457 & 12.325 & $\mathbf{2 4 . 7 8 2}$ \\
\hline $45-64$ & 15.389 & 15.874 & $\mathbf{3 1 . 2 6 3}$ \\
\hline$>65$ & 11.013 & 13.566 & $\mathbf{2 4 . 5 7 6}$ \\
\hline
\end{tabular}

(C) Memòria d'activitat 1996 de la Regió Sanitària Tortosa [1997, p. 29].

La población entre 0 -14 años representa el 17,37\% de la población total, mientras que la población mayor de 65 años representa el 18,63\%. Por sexos, el 49,59\% de la población son hombres y el 50,41\%, mujeres. El número de hombres es más elevado en los grupos de edades hasta los 29 años, esta diferencia se equilibra en el grupo de 30-44 años, en cambio, en el grupo de mayores de 65 años existe una diferencia de 3,6 puntos favorable al grupo de mujeres. Con relación a la pirámide de población, ésta refleja claramente el proceso de envejecimiento de la población y de reducción de la natalidad.

En cuanto a los sectores de ocupación, en la comarca del Baix Ebre existe un predominio del sector servicios, con el 46,18\% de la población 
ocupada, mientras que en el Montsià es del 37,5\%. El sector de la agricultura, ganadería y pesca, ocupa el primer lugar en la comarca de la Terra Alta, con el 39,79\% de la población ocupada, el Montsià se encuentra en el segundo lugar, con el 25,74\%, y el Baix Ebre en el tercero, con el 17,96\%. En total, la población dedicada a este sector representa el 12,94\% de la población que se dedica en toda Catalunya. En los sectores de la construcción y la industria los porcentajes de población ocupada son muy similar en las tres comarcas. Con relación a los datos económicos, la renta per cápita de las comarcas de la RSTO es inferior al del total de Catalunya y también al de la provincia de Tarragona.

TABLA 6.23. Renta bruta familiar disponible, RSTO.

\begin{tabular}{|l|r|r|r|r|}
\hline \multicolumn{2}{|c|}{ Baix Ebre } & Montsià & Terra Alta & Total \\
\hline Total [millones Ptas.] & 68.490 & 55.119 & 12.089 & $\mathbf{1 3 5 . 6 9 8}$ \\
\hline Per cápita [miles Ptas.] & $1.059,5$ & $1.015,0$ & 933,9 & $\mathbf{1 . 0 2 8 , 8}$ \\
\hline
\end{tabular}

(C) Memòria d'activitat 1996 de la Regió Sanitària Tortosa [1997, p. 30].

\subsubsection{SECTORES SANITARIOS Y ABS}

Los SS son subunidades territoriales integrados en las RS y son formados por un conjunto de áreas básicas de salud. En su ámbito se desarrollan y se coordinan las actividades de prevención de la enfermedad, promoción de la salud y salud pública, la asistencia sanitaria y sociosanitaria en el nivel de atención primaria, y las especialidades médicas de soporte y referencia de ésta. 
FIGURA 6.26. Mapa de la RSTO.

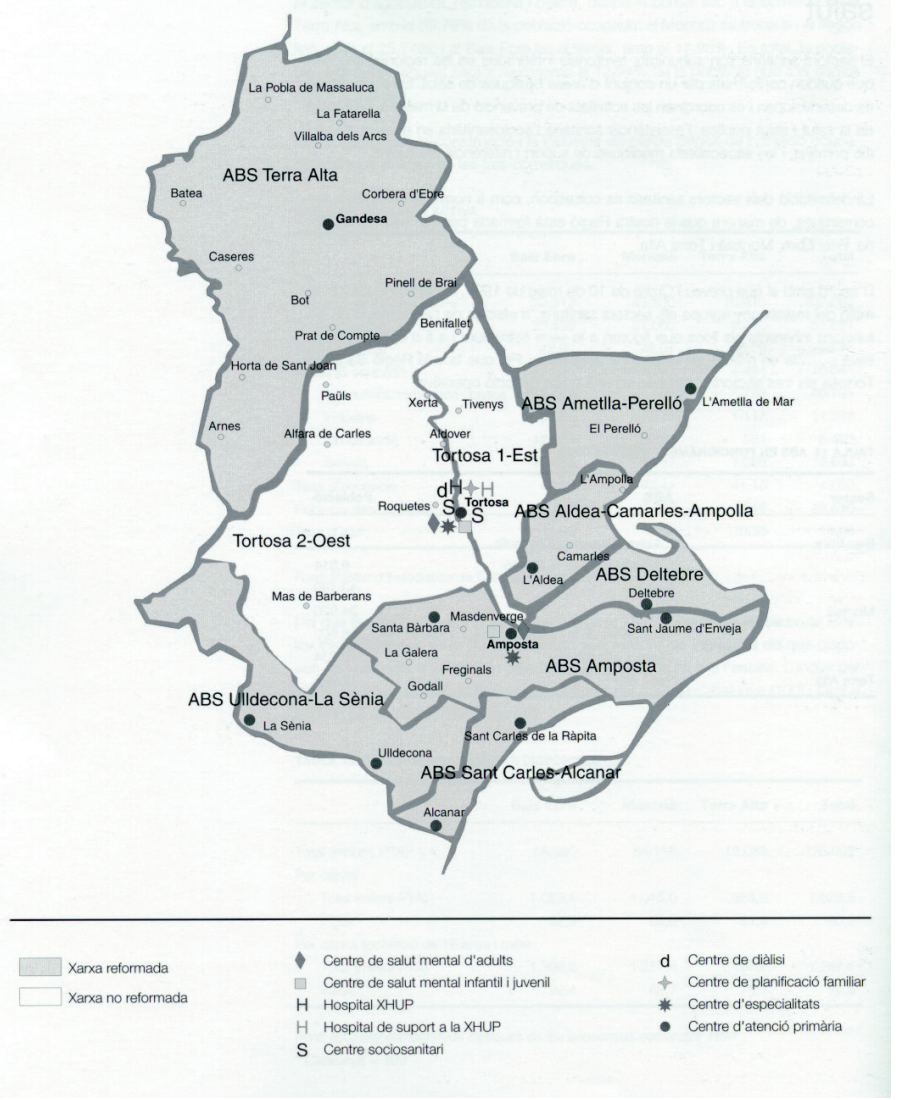

(C) Memòria d'activitat 1996 de la Regió Sanitària Tortosa [1997, p. 32].

La delimitación de los sectores sanitarios se corresponde, como norma general, con las comarcas, de manera que la RSTO está formada por tres sectores sanitarios:

- Baix Ebre.

- Montsià.

- Terra Alta.

De acuerdo con el que prevé la Orden de 10 de mayo de 1994, la Resolución de 12 de mayo del mismo año agrupa los sectores sanitarios, a efectos de la acumulación de las funciones inherentes a los lugares que figuran en su estructura y con objetivo de agilizar su puesta en marcha, en direcciones operativas.

Con relación a la RSTO los tres sectores se agrupan en una única dirección operativa. 
TABLA 6.24. ABS en funcionamiento, por sectores RSTO.

\begin{tabular}{|l|l|}
\hline SS & ABS \\
\hline Ba ix Ebre & $\begin{array}{l}\text { L'Aldea, Camarles, I'Ampolla, L'Ametla de Mar, el Perelló, } \\
\text { Deltebre. }\end{array}$ \\
\hline Montsià & $\begin{array}{l}\text { Amposta, Sant Carles de la Ràpita, Alcanar, Ulldecona, } \\
\text { La Sénia. }\end{array}$ \\
\hline Terra Alta & Terra Alta \\
\hline
\end{tabular}

(C) Memòria d'activitat 1996 de la Regió Sanitària Tortosa [1997, p. 31].

\subsubsection{ESTRUCTURA CORPORATIVA}

La LOSC establece que los órganos de dirección y gestión de las Regiones Sanitarias son el Consejo de Dirección, los gerentes y los órganos u organismos, los servicios y las unidades que se constituyen por reglamentación y, así mismo, que el Consejo de Salud es el órgano de participación en la RS —artículo 24 de la LOSC en anexo-.

Con relación a las unidades, el Comité Directivo del SCS acordó —en consecución de las funciones encomendadas a las regiones sanitarias- prever cinco ámbitos funcionales de actuación:

- Planificación.

- Compra de servicios.

- Atención al cliente.

- Control de gestión.

- Soporte operativo.

FIGURA 6.27. Organigrama de la RSTO.

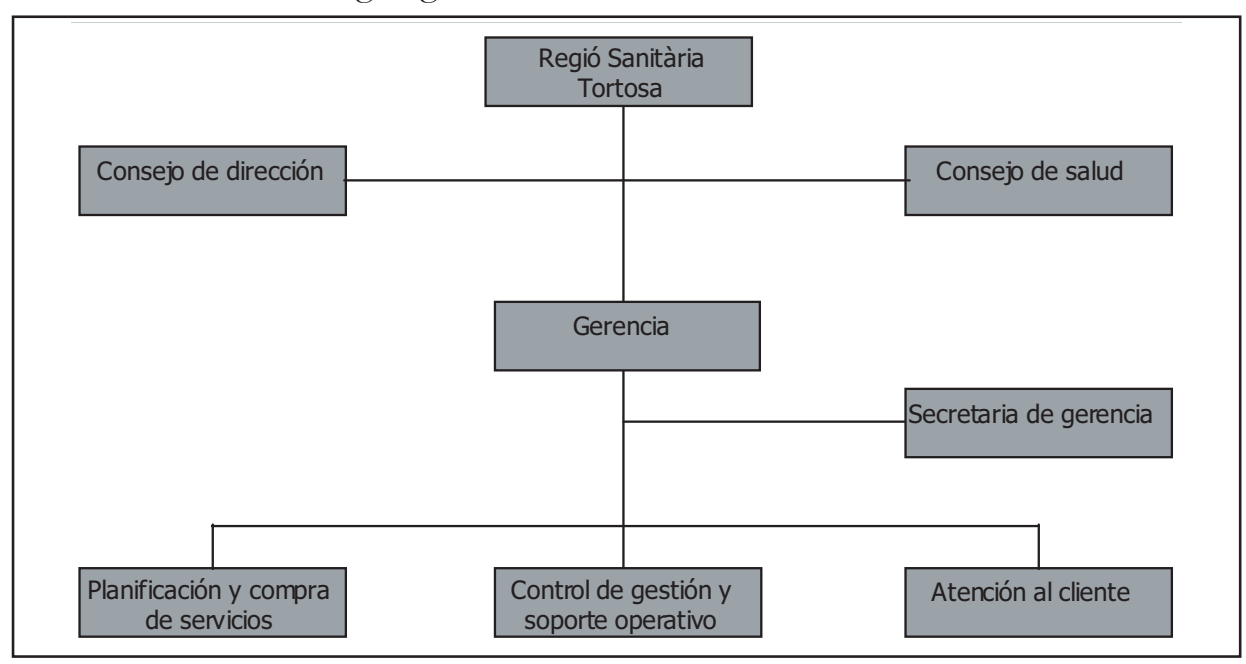

(C) Memòria d'activitat 1996 de la Regió Sanitària Tortosa [1997, p. 41]. 
En la RSTO, ateniéndose a sus propias características, estos ámbitos funcionales se agruparon en tres unidades:

- Planificación y Compra de Servicios.

- Atención al Cliente.

- Control de Gestión y Soporte Operativo.

\subsection{REGIÓ SANITÀRIA GIRONA}

\subsubsection{DESCRIPCIÓN, TERRITORIO Y POBLACIÓN}

El ámbito territorial de la Regió Sanitària Girona — RSG - fue establecido por el Decreto 114/1994, de 3 de mayo, de delimitación de las regiones sanitarias y de los sectores sanitarios del Servei Català de la Salut — SCS_, y comprende los siguientes sectores sanitarios:

- Gironès.

- Selva.

- Pla de l'Estany.

- Baix Empordà.

- Alt Empordà.

- Garrotxa.

- Ripollès.

Estos sectores sanitarios prácticamente coinciden con las comarcas de los mismos nombres, con excepción de algunos municipios limítrofes, que están adscritos a las áreas básicas de salud del sector sanitario vecino.

Hasta la entrada en vigor del comentado Decreto, la RSG incluya también la parte de la Cerdanya correspondiente a la provincia de Girona, y a efectos de gestión se mantuvo este ámbito hasta finales del año 1994.

La población total de aquel ámbito era de 509.628 personas, según los datos del censo de 1991.

En el período 1981-1991 se produzco una tasa de crecimiento demográfico del $9,2 \%$, muy superior a la del conjunto de Catalunya - $1,7 \%$ -

El crecimiento vegetativo anual, equivalente a la diferencia entre la tasa de natalidad y la tasa de mortalidad, solamente explicaba una quinta parte de este crecimiento. El resto del crecimiento se ha de atribuir a un saldo migratorio positivo. 
TABLA 6.25. Pirámide poblacional, censo de 1991 de la RSG.

\begin{tabular}{|l|r|r|r|r|r|r|}
\hline \multirow{2}{*}{ Grupo de edad } & \multicolumn{3}{|c|}{ Número de personas } & \multicolumn{2}{c|}{ Proporción sobre la población } \\
\cline { 2 - 7 } & Hombres & Mujeres & Total & Hombres & Mujeres & Total \\
\hline $0-14$ & 47.240 & 44.502 & 91.742 & $9,47 \%$ & $8,92 \%$ & $18,40 \%$ \\
\hline $15-29$ & 59.597 & 57.231 & 116.858 & $11,95 \%$ & $11,48 \%$ & $23,43 \%$ \\
\hline $30-44$ & 53.752 & 51.393 & 105.145 & $10,78 \%$ & $10,31 \%$ & $21,08 \%$ \\
\hline $45-64$ & 53.659 & 54.279 & 107.938 & $10,76 \%$ & $10,88 \%$ & $21,64 \%$ \\
\hline$>65$ & 33.254 & 43.810 & 77.064 & $6,67 \%$ & $8,78 \%$ & $15,45 \%$ \\
\hline Total & $\mathbf{2 4 7 . 5 0 2}$ & $\mathbf{2 5 1 . 2 1 5}$ & $\mathbf{4 9 8 . 7 1 7}$ & $\mathbf{4 9 , 6 3 \%}$ & $\mathbf{5 0 , 3 7 \%}$ & $\mathbf{1 0 0 , 0 0 \%}$ \\
\hline
\end{tabular}

(C) Memòria d'activitat 1996 de la Regió Sanitària Girona [1997, p. 28].

La distribución de la población por grupos de edades y sexo se estrecha en los grupos más jóvenes e se ensancha en los de mayor edad. Comparativamente la proporción de mayores de 65 años - 15,5\% — es superior a la global de Catalunya — 14,3\% — . La distribución de la población de la RSG es bastante dispersa, quedando claro en la densidad de la población — 190 habitantes por $\mathrm{Km}^{2}$ — , y en el gran número de municipios que comprende —209_, 100 de los cuales son de menos de 500 habitantes.

TABLA 6.26. Indicadores sociodemográficos de la RSG.

\begin{tabular}{|l|r|r|r|}
\hline \multicolumn{2}{|c}{ RSG } & \multicolumn{1}{c|}{ Catalunya } & Girona/Catalunya \\
\hline Extensión & 5.517 & $31.895,3 \mathrm{Km}^{2}$ & $17,30 \%$ \\
\hline Población & 519.368 & 6.090 .040 Habitantes & $8,53 \%$ \\
\hline Densidad población & 94,1 & 190,9 Habitantes/ Km ${ }^{2}$ & \\
\hline Menores de 15 años & 15,66 & $15 \%$ & $8,40 \%$ \\
\hline Mayores de 65 años & 16,83 & $16 \%$ & \\
\hline Población activa & 241,9 & $2.663,6$ Personas & \\
\hline Renta per cápita & $1.524,5$ & $1.363,3$ Miles PTA & \\
\hline
\end{tabular}

(C) Memòria d'activitat 1997 de la Regió Sanitària Girona [1998, p. 26].

\subsubsection{SECTORES SANITARIOSY ABS}

El eje vertebrador del sistema sanitario es el área básica de salud —ABS—, unidad territorial elemental donde se presta la atención primaria de salud de acceso directo de la población —-LOSC, artículo 41 en anexo-. 
Las ABS se delimitan según factores geográficos, demográficos, sociales, epidemiológicos y de vías de comunicación homogéneas.

Cada ABS cuenta, como mínimo, con un centro de atención primaria - CAP_ y se ha de coordinar con todos los servicios sanitarios y sociosanitarios de atención primaria en su ámbito, como también con los servicios jerarquizados de especialidades y los hospitales que tengan asignados.

TABLA 6.27. Distribución territorial de la RSG.

\begin{tabular}{|l|l|l|}
\hline $\begin{array}{l}\text { Dirección } \\
\text { operativa }\end{array}$ & Sector Sanitario & Área básica de salud \\
\hline Dirección operativa 5 & Baix Empordà & $\begin{array}{l}\text { La Bisbal d'Empordà, Palafrugell, Palamós, } \\
\text { Sant Feliu de Guíxols, Torroella de Montgrí. }\end{array}$ \\
\cline { 2 - 3 } & Gironès & $\begin{array}{l}\text { Cassà de la Selva, Celrà, Girona 1, Girona 2, Girona 3, } \\
\text { Girona 4, Salt, Sarrià de Ter. }\end{array}$ \\
\cline { 2 - 3 } & Pla de l'Estany & Banyoles \\
\cline { 2 - 3 } & Selva & $\begin{array}{l}\text { Anglès, Arbúcies-Sant Hilari Sacalm, Blanes, } \\
\text { Breda-Hostalric, Lloret de Mar, Santa Coloma de Farners, } \\
\text { Sils-Vidreres-Maçanet de la Selva. }\end{array}$ \\
\hline Dirección operativa 6 & Alt Empordà & $\begin{array}{l}\text { Bàscara, L'Escala, Figueres, La Jonquera, Llançà, } \\
\text { Peralada, Roses, Vilafant. }\end{array}$ \\
\cline { 2 - 3 } & Garroxta & Besalú, Olot, Sant Joan les Fonts, La Vall d'en Bas. \\
\cline { 2 - 3 } & Ripollès & Camprodon, Ribes de Freser-Campdevànol, Ripoll. \\
\hline
\end{tabular}

Fuente: Elaboración propia a partir de la Memòria d'activitat 1996 de la Regió Sanitària Girona [1997].

Las funciones de la $\mathrm{ABS}$ se desarrollan cuando se crea el equipo de atención primaria - EAP_, formado por un conjunto de profesionales sanitarios y no sanitarios, que actúan de manera integrada, por medio del trabajo en equipo. La región sanitaria se ha de ordenar en subunidades territoriales denominados sectores sanitarios - SS-.

El ámbito territorial de los sectores fue establecido por el Decreto 114/1994, de 3 de mayo, de delimitación de las regiones sanitarias y de los sectores sanitarios del SCS, y prácticamente coinciden con los territorios de las comarcas. 
FIGURA 6.28. Mapa de la RSG.

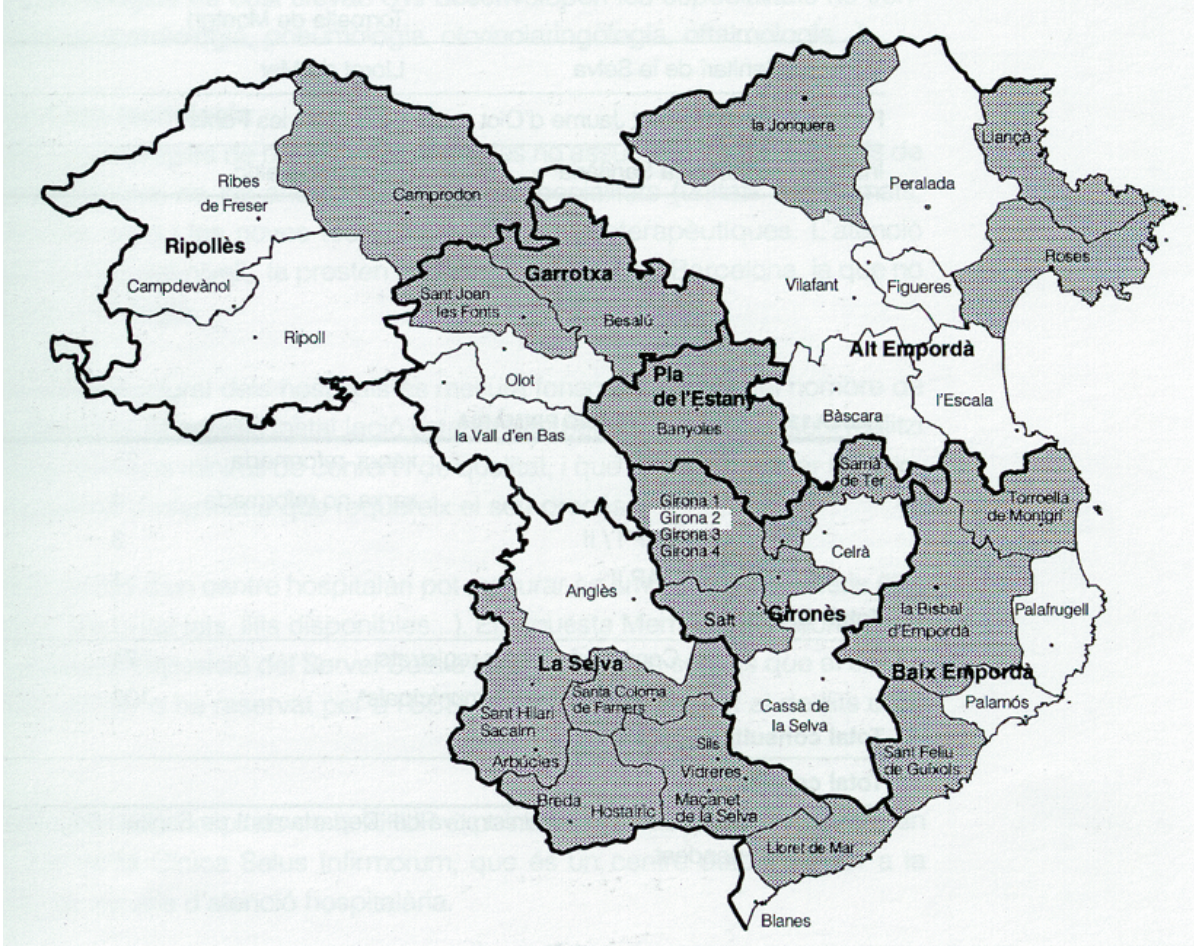

(C) Memòria d'activitat 1996 de la Regió Sanitària Girona [1997, p. 33].

\subsubsection{ESTRUCTURA CORPORATIVA}

La RSG se estructura, de acuerdo con el artículo 24 y siguientes de la LOSC - en anexo-, en diversos órganos de dirección y gestión y un órgano de participación.

El gerente asume la dirección y la gestión de la Regió Sanitària, como también la representación plena del Consejo de Dirección con relación a la ejecución de los acuerdos que esta adopte.

El gerente tiene el soporte de las unidades que se ven en el organigrama. 
FIGURA 6.29. Organigrama de la RSG.

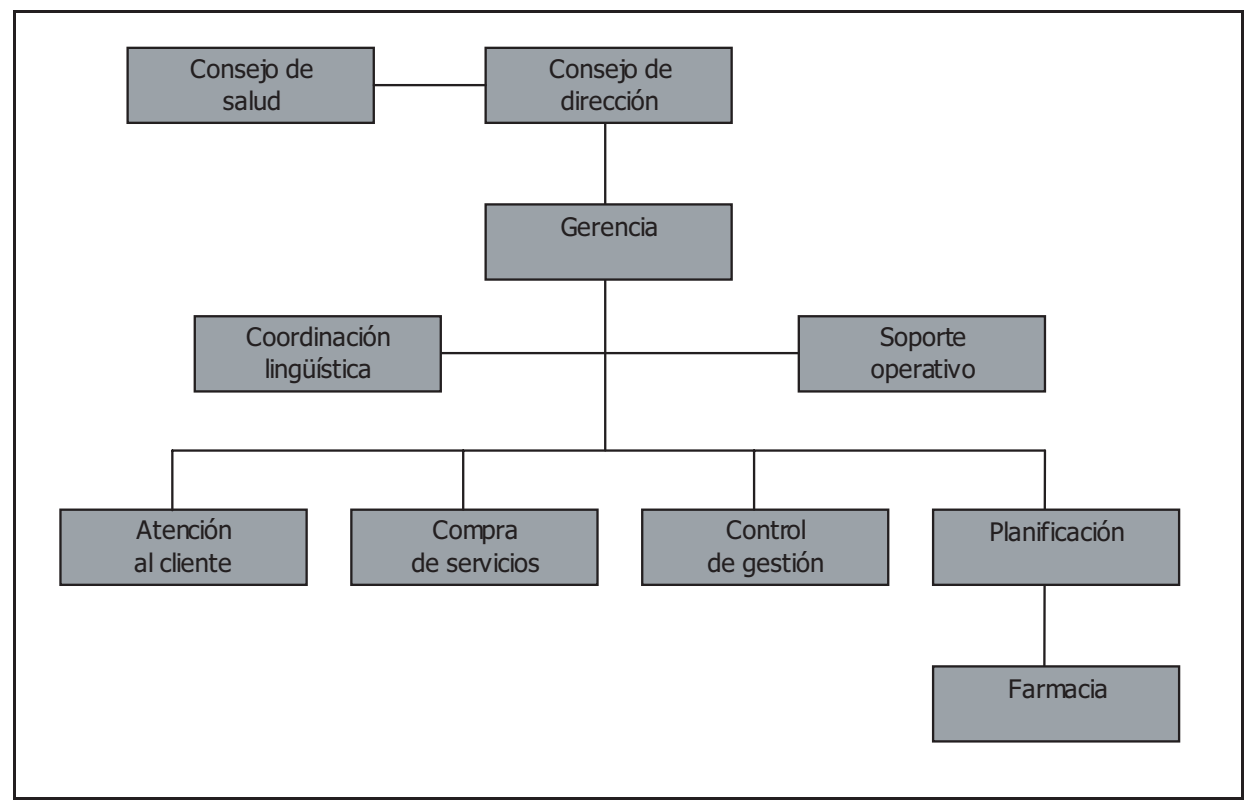

(C) Memòria d'activitat 1996 de la Regió Sanitària Girona [1997, p. 46].

\subsection{REGIÓ SANITÀRIA COSTA DE PONENT}

\subsubsection{DESCRIPCIÓN, TERRITORIO Y POBLACIÓN}

La Regió Sanitària Costa de Ponent — RSCP— está formada por siete sectores sanitarios, que son los siguientes:

- Barcelonès Sud l'Hospitalet de Llobregat

- Baix Llobregat Centre-Nord

- Baix Llobregat Fontsanta

- Baix Llobregat Delta—Litoral

- Garraf

- Alt Penedès

- Anoia

La distribución demográfica de la RSCP es la siguiente: 
TABLA 6.28. Distribución demográfica de la RSCP.

\begin{tabular}{|l|r|l|}
\hline Extensión & 2.089 & $\mathrm{Km}^{2}$ \\
\hline Población & 1.115 .098 & Habitantes \\
\hline Población activa & 411.335 & Habitantes \\
\hline Tasa de natalidad & 9,12 & Por mil habitantes \\
\hline Tasa de mortalidad & 7,16 & Por mil habitantes \\
\hline Esperanza de vida & $\begin{array}{r}\text { Hombres } \\
\text { Mujeres }\end{array}$ & $\begin{array}{l}74,1 \text { años } \\
81,7 \text { años }\end{array}$ \\
\hline
\end{tabular}

(C) Memòria d'activitat 1996 Regió Sanitària Costa de Ponent [1997, p. 29].

TABLA 6.29. Habitantes por grupos de edad, censo 1991 de la SCP.

\begin{tabular}{|l|r|r|r|r|}
\hline Sector sanitario & $\mathbf{0 - 1 4}$ & $\mathbf{1 5} \mathbf{- 6 4}$ & $\mathbf{7 6 5}$ & Total \\
\hline Anoia & 15.648 & 53.330 & 11.632 & 80.610 \\
\hline Barc. Sud I'Hospitalet de Llobregat & 46.648 & 194.827 & 31.103 & 272.578 \\
\hline Baix Llobregat Fontsanta & 29.291 & 122.757 & 18.637 & 170.685 \\
\hline Baix Llobregat Centre-Nord & 31.867 & 109.046 & 17.689 & 158.602 \\
\hline Baix Llobregat Deta-Litoral & 61.824 & 201.743 & 25.737 & 289.304 \\
\hline Garraf & 15.033 & 53.691 & 10.616 & 79.340 \\
\hline Alt Penedès & 11.514 & 41.954 & 10.511 & 63.979 \\
\hline Total RSCP & $\mathbf{2 1 1 . 8 2 5}$ & $\mathbf{7 7 7 . 3 4 8}$ & $\mathbf{1 2 5 . 9 2 5}$ & $\mathbf{1 . 1 1 5 . 0 9 8}$ \\
\hline
\end{tabular}

(C) Memòria d'activitat 1996 Regió Sanitària Costa de Ponent [1997, p. 30]. 


\subsubsection{SECTORES SANITARIOSY ABS}

FIGURA 6.30. Mapa de la RSCP.

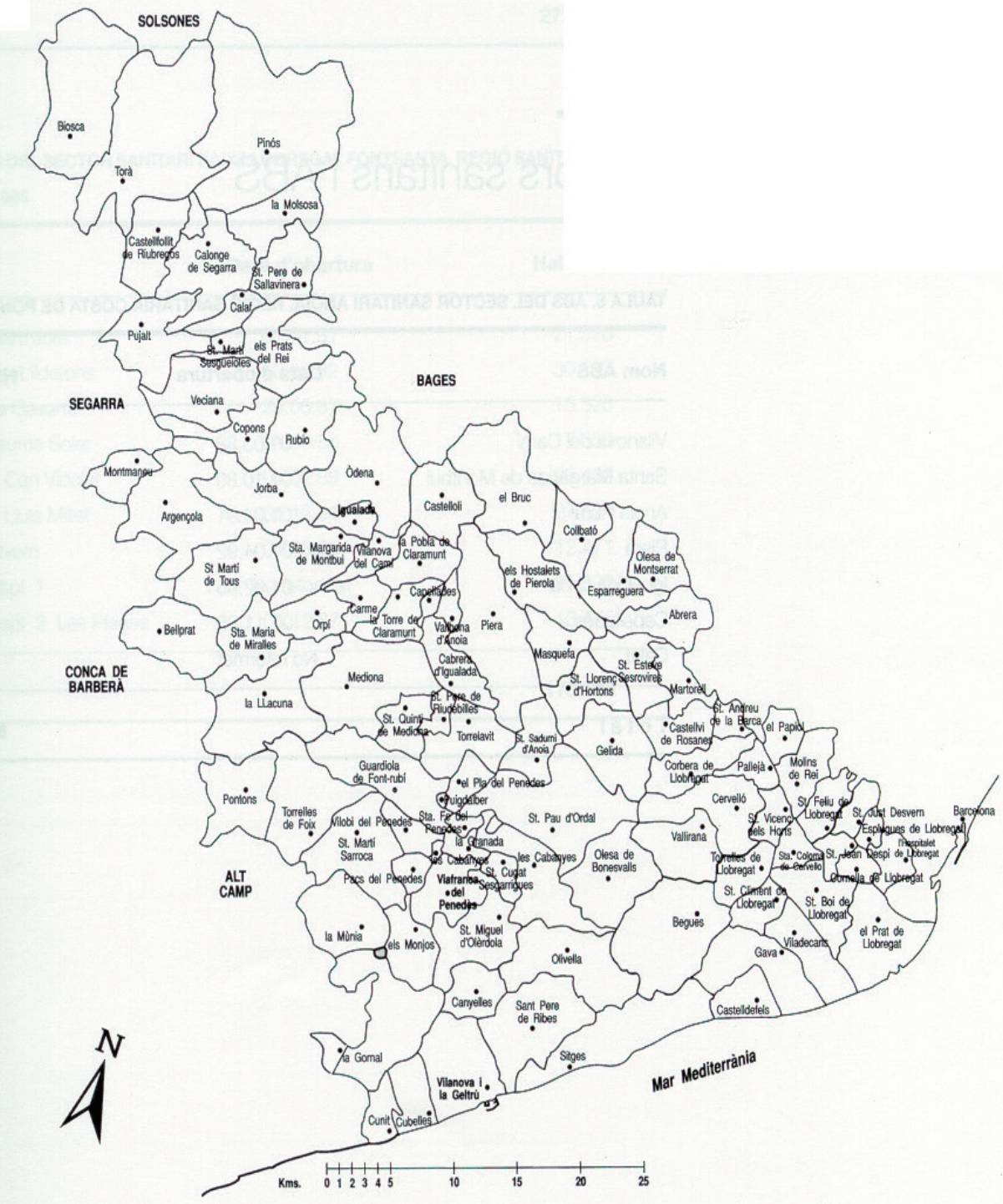

(C) Memòria d'activitat 1996 Regió Sanitària Costa de Ponent [1997, p. 29]. 
TABLA 6.30. Distribución territorial de la RSCP.

\begin{tabular}{|l|l|l|l|}
\hline SS & ABS & SS & ABS \\
\hline Anoia & $\begin{array}{l}\text { Vilanova del Camí, Sta Margarida } \\
\text { de Montbui, Anoia Rural, Piera, } \\
\text { Igualada Urbà, Capellades, Calaf. }\end{array}$ & $\begin{array}{l}\text { Baix } \\
\text { Llobregat } \\
\text { Fontsanta }\end{array}$ & $\begin{array}{l}\text { Cornellà 1, Cornellà 2, Cornellà 3, } \\
\text { Cornellà 4, Espluges 1, Espluges 2, } \\
\text { Sant Just Desvern, Sant Joan Despí 1, } \\
\text { Sant Joan Despí 2. }\end{array}$ \\
\hline $\begin{array}{l}\text { Barcelonès } \\
\text { Sud } \\
\text { L'Hospitalet } \\
\text { de Llobregat }\end{array}$ & $\begin{array}{l}\text { L'Hospitalet 1, L'Hospitalet 2, } \\
\text { L'Hospitalet 3, L'Hospitalet 4, } \\
\text { L'Hospitalet 5, L'Hospitalet 6, } \\
\text { L'Hospitalet 7, L'Hospitalet 8, } \\
\text { L'Hospitalet 9, L'Hospitalet 10, } \\
\text { L'Hospitalet 11, L'Hospitalet 12, } \\
\text { L'Hospitalet 13. }\end{array}$ & $\begin{array}{l}\text { Baix } \\
\text { Llobregat } \\
\text { Centre- } \\
\text { Nord }\end{array}$ & $\begin{array}{l}\text { Molins de Rei, Vallirana, Martorell, } \\
\text { Esparreguera, Olesa de Montserrat, } \\
\text { Sant Andreu de la Barca, } \\
\text { Sant Feliu de Llobregat 1, } \\
\text { Sant Feliu de Llobregat 2. }\end{array}$ \\
\hline $\begin{array}{l}\text { Baix } \\
\text { Llobregat } \\
\text { Delta-Litoral }\end{array}$ & $\begin{array}{l}\text { El Prat 1, El Prat 2, El Prat 3, } \\
\text { Sant Boi 1, Sant Boi 2, } \\
\text { Sant Boi 3, Sant Boi 4, } \\
\text { Sant Vicenç dels Horts, } \\
\text { Viladecans 1, Viladecans 2, } \\
\text { Castelldefels, Gavà 1, Gavà 2. }\end{array}$ & Garraf & $\begin{array}{l}\text { Garraf Rural, Sitges, } \\
\text { Vilanova i la Gertrú 1, } \\
\text { Vilanova i la Gertrú 2. }\end{array}$ \\
\cline { 2 - 4 } & $\begin{array}{l}\text { Alt Penedès } \\
\text { Venedès Rural, } \\
\text { Vilafranca del Penedès, } \\
\text { Sant Sadurní d'Anoia. }\end{array}$ \\
\hline
\end{tabular}

Fuente: Elaboración propia a partir de la Memòria d'activitat 1996 Regió Sanitària Costa de Ponent [1997].

\subsubsection{ESTRUCTURA CORPORATIVA}

La RSCP tiene totalmente desplegada la estructura funcional, tanto con relación a los servicios centrales como con la estructura del territorio.

FIGURA 6.31. Organigrama de la RSCP.

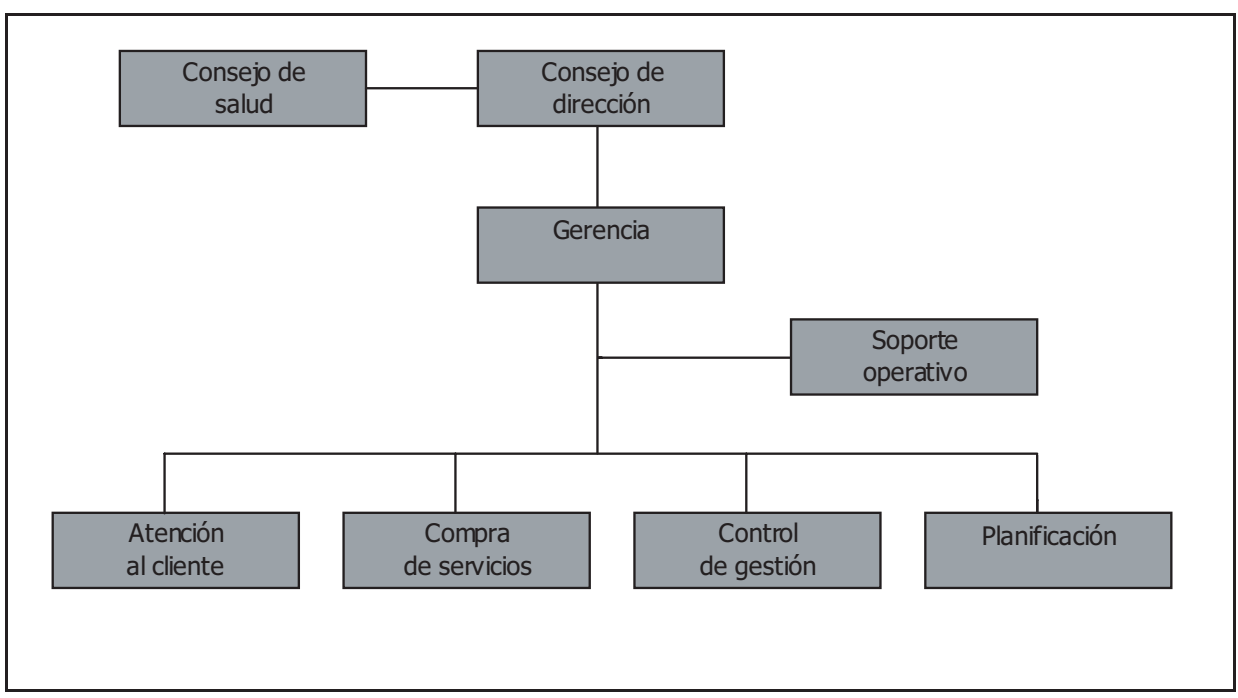

Fuente: Elaboración propia a partir de la Memòria d'activitat 1996 Regió Sanitària Costa de Ponent [1997]. 


\subsection{REGIÓ SANITÀRIA BARCELONÈS NORD I MARESME}

\subsubsection{DESCRIPCIÓN, TERRITORIO Y POBLACIÓN}

La Regió Sanitària Barcelonès Nord i Maresme — RSBNM — es la responsable del funcionamiento del sistema sanitario público en el territorio delimitado por las ciudades de Badalona, Sant Adrià de Besòs y Santa Coloma de Gramenet — Barcelonès Nord_ y la comarca del Maresme.

TABLA 6.31. Resumen de las características de la RSBNM.

\begin{tabular}{|c|c|c|c|}
\hline \multicolumn{3}{|l|}{ Datos estructurales } & \multirow[t]{2}{*}{ Definición parámetro } \\
\hline Población & $\begin{array}{l}\text { Barcelonès Nord } \\
\text { Maresme } \\
\text { Total }\end{array}$ & $\begin{array}{l}367.523 \\
318.891 \\
686.414\end{array}$ & \\
\hline Extensión & $\begin{array}{l}\text { Barcelonès Nord } \\
\text { Maresme } \\
\text { Total }\end{array}$ & $\begin{array}{r}32,44 \\
397,10 \\
429,50\end{array}$ & $\mathrm{Km}^{2}$ \\
\hline Densidad de población & $\begin{array}{l}\text { Barcelonès Nord } \\
\text { Maresme } \\
\text { Total }\end{array}$ & $\begin{array}{r}11.329,3 \\
803,0 \\
1.589,1\end{array}$ & Habitantes por $\mathrm{Km}^{2}$ \\
\hline División territorial & & 2 & Número de comarcas \\
\hline Tasa de mortalidad & & 6,95 & Defunciones por mil habitantes \\
\hline Tasa de natalidad & & 9,61 & Nacimientos por mil habitantes \\
\hline
\end{tabular}

(C) Memòria d'activitat 1996 RSBNM [1997, p. 31].

En la RSBNM residen oficialmente, según datos del censo de 1996, 686.414 habitantes. La estructura de la población es bastante joven, independiente de apreciarse una caída de la natalidad desde el año 1975, igual al resto de Catalunya.

\subsubsection{SECTORES SANITARIOS Y ABS}

\subsubsection{SECTOR SANITARI BARCELONÈS NORD}

Este SS está formado por el conjunto de tres municipios:

- Badalona.

- Sant Adrià de Besòs.

- Santa Coloma de Gramenet. 
Según el censo de 1991, tienen un total de 386.016 habitantes. Durante el período de expansión demográfica este sector experimenta un incremento muy intenso de su población.

El municipio de Santa Coloma de Gramenet, por ejemplo, pasó de tener 32.950 habitantes en el año 1950 a tener 137.579 en el año 1975. Esta masiva inmigración es la responsable de la característica más destacada desde el punto de vista demográfico — la juventud de su población-.

El carácter preferentemente industrial de la inmigración hizo que la crisis económica afectase especialmente este sector, de forma que algunos municipios presentan, desde el año 1977, saldos migratorios negativos.

Según datos del censo de 1991:

- El 10,28\% de la población del Barcelonès Nord tiene más de 64 años.

- El Barcelonès Nord es un de los SS con una población de las más jóvenes de Catalunya. Tiene un índice de envejecimiento del 0,52, que contrasta con el 0,80 de Catalunya.

- La tasa de natalidad —9,05/1.000 habitantes—y la global de fertilidad —33,80/1.000 habitantes - del Barcelonès Nord, que hasta ahora eron superiores a los de Catalunya, ya es superada por los datos globales catalanes — 9,3/1.000 habitantes y 36,5/1.000 habitantesrespectivamente.

- La densidad de la población es extremadamente elevada —11.329 habitantes $/ \mathrm{km}^{2}$ -

TABLA 6.32. Evolución demográfica de los municipios del Barcelonès Nord.

\begin{tabular}{|l|r|r|r|r|r|r|}
\hline \multicolumn{3}{c}{ Población } & \multicolumn{1}{c|}{ Crecimiento absoluto } \\
\hline Municipio & \multicolumn{1}{|c|}{$\mathbf{1 9 8 6}$} & \multicolumn{1}{c|}{$\mathbf{1 9 9 1}$} & \multicolumn{1}{c|}{$\mathbf{1 9 9 6}$} & $\mathbf{8 6 / 9 1}$ & \multicolumn{1}{|c|}{$\mathbf{9 1 / 9 6}$} & \multicolumn{1}{|c|}{$\mathbf{8 6 / 9 6}$} \\
\hline Badalona & 225.016 & 218.725 & 210.987 & -6.291 & -7.738 & -14.029 \\
\hline Sant Adrià de Besòs & 34.735 & 34.154 & 33.361 & -581 & -793 & -1.374 \\
\hline Santa Coloma & 135.258 & 133.138 & 123.175 & -2.120 & -9.963 & -12.083 \\
\hline Total SS & $\mathbf{3 9 5 . 0 0 9}$ & $\mathbf{3 8 6 . 0 1 7}$ & $\mathbf{3 6 7 . 5 2 3}$ & $\mathbf{- 8 . 9 9 2}$ & $\mathbf{- 1 8 . 4 9 4}$ & $\mathbf{- 2 7 . 4 8 6}$ \\
\hline
\end{tabular}

(C) Memòria d'activitat 1996 Regió Sanitària Barcelonès Nord i Maresme [1997, p. 32]. 
TABLA 6.33. Distribución territorial del SS Barcelonès Nord.

\begin{tabular}{|l|l|}
\hline Sector sanitario & ABS \\
\hline Barcelonès Nord & Badalona 1, Badalona 2, Badalona 3, Badalona 4, Badalona 5, \\
& Badalona 6, Badalona 7A, Badalona 7B, Badalona 8, Badalona 9, \\
& Badalona 10, Badalona 11, Badalona 12, Sant Adrià 1, Sant Adrià 2, \\
& Santa Coloma de Gramenet 1, Santa Coloma de Gramenet 2, \\
& Santa Coloma de Gramenet 3, Santa Coloma de Gramenet 4, \\
& Santa Coloma de Gramenet 5, Santa Coloma de Gramenet 6. \\
\hline
\end{tabular}

Fuente: Elaboración propia a partir de Memòria d’activitat 1996 Regió Sanitària Barcelonès Nord i Maresme [1997].

\subsubsection{SECTOR SANITARI EL MARESME}

El SS El Maresme comprende la comarca geográfica del Maresme y el municipio de Fogars de Tordera. La capital es Mataró. En la evolución de la población del Maresme desde el año 1950 hasta hoy se distingue claramente dos fases: una primera, de fuerte expansión demográfica — año 1950 hasta 1975- - y una segunda —desde el 1975 hasta la actualidad— de crecimiento más moderado, si bien las dos etapas tienen niveles de crecimiento superiores a los de Catalunya.

El saldo inmigratorio entre el 1950 y el 1986 se calcula en unas 108.000 personas. Este fenómeno ha tenido un efecto rejuvenecedor sobre la pirámide de población y explica, en parte, unas tasas de natalidad superiores a la media catalana.

Los rasgos más característicos de la población del Maresme, referidos en el censo del año 1991, son los siguientes:

- El 19,9\% de la población tiene de 0 a 14 años, los mayores de 65 años representan el 12,32\%.

- El índice de envejecimiento es del 0,62, por debajo del 0,80 de Catalunya.

- La relación de dependencia global — 47,52 — es ligeramente superior a la media catalana -47,22—, fundamentalmente por la relación superior de dependencia juvenil, ya que la senil es inferior.

- Las tasas de natalidad — 10,5/1.000 habitantes—y global de fertilidad — 40,2/1.000 habitantes— son más altas en el Maresme que en Catalunya — 9,3 y 36,5/1.000 habitantes - respectivamente.

La evolución del censo en El Maresme durante los últimos diez años muestra un aumento de 49.096 habitantes, cifra que supone un incremento del 18\%.

El precio de la vivienda en Barcelona, las obras viarias realizadas y las que se encuentron en fase de ejecución podrían justificar el mantenimiento de la 
tendencia actual que no parece, ahora, haber llegado al limite. Esto puede suponer, para el año 2000, una población de más de 360.000 habitantes.

TABLA 6.34. Evolución demográfica del SS El Maresme.

\begin{tabular}{|l|c|c|c|c|c|c|}
\hline \multicolumn{3}{|c}{ Población } & $\mathbf{8 1}$ Crecimiento absoluto \\
\hline SS Maresme & $\mathbf{1 9 8 6}$ & $\mathbf{1 9 9 1}$ & $\mathbf{1 9 9 6}$ & $\mathbf{8 6 / 9 1}$ & $\mathbf{9 1 / 9 6}$ & $\mathbf{8 6 / 9 6}$ \\
\hline Total SS & 269.795 & 293.500 & 318.891 & 23.705 & 25.788 & 49.493 \\
\hline
\end{tabular}

Fuente: Elaboración propia a partir Memòria d'activitat 1996 Regió Sanitària Barcelonès Nord i Maresme [1997].

TABLA 6.35. Distribución territorial del SS El Maresme.

\begin{tabular}{|l|l|}
\hline Sector sanitario & ABS \\
\hline El Maresme & Arenys de Mar, Sant Andreu de Llavaneres, Argentona, Calella, \\
& $\begin{array}{l}\text { Pineda de Mar, Canet de Mar, Malgrat de Mar, Tordera, Premià, } \\
\text { El Masnou, Mongat, Vilassar de Dalt, Vilassar de Mar, Mataró 1, } \\
\text { Mataró 2, Mataró 3, Mataró 4, Mataró 5, Mataró 6, Mataró 7. }\end{array}$ \\
\hline
\end{tabular}

Fuente: Elaboración propia a partir de Memòria d'activitat 1996 Regió Sanitària Barcelonès Nord i Maresme [1997].

FIGURA 6.32. Mapa de la RSBNM.

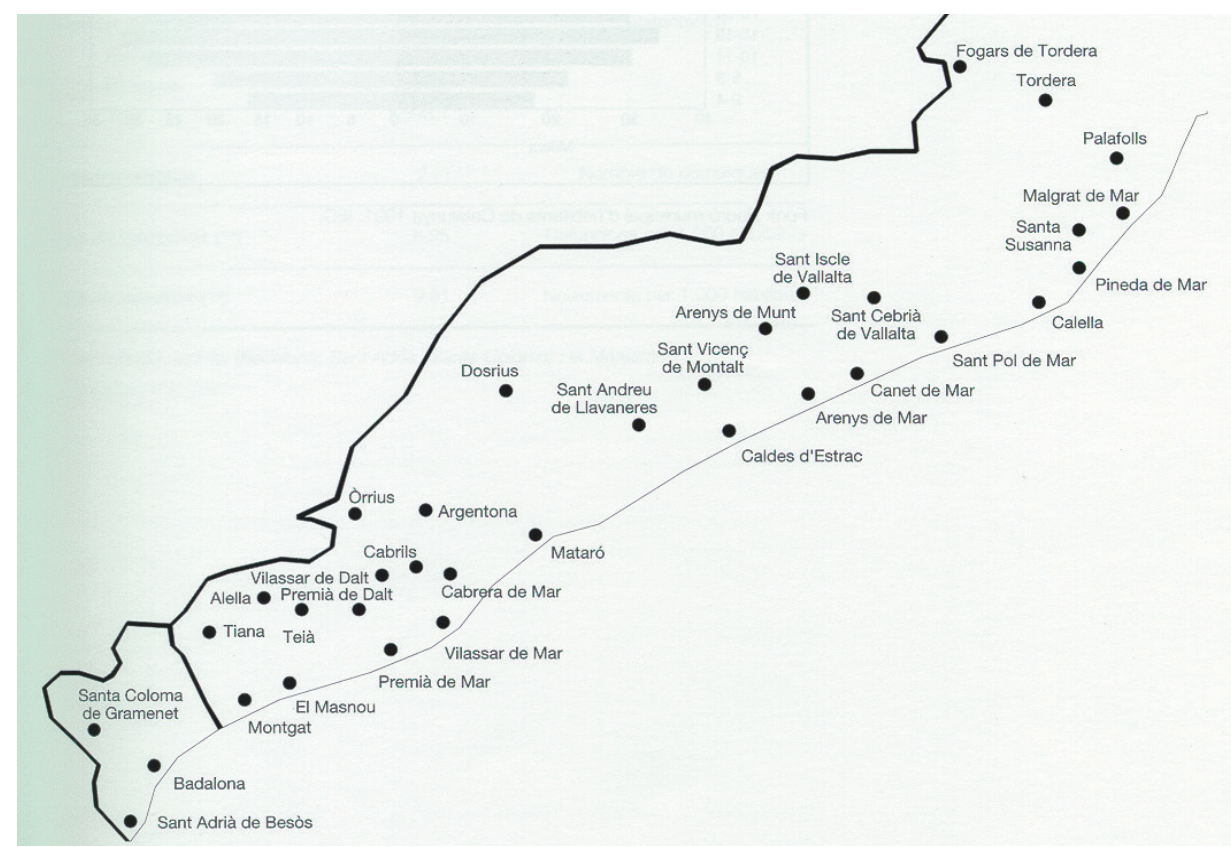

(C) Memòria d'activitat 1996 Regió Sanitària Barcelonès Nord i Maresme [1997, p. 29]. 


\subsubsection{ESTRUCTURA CORPORATIVA}

La RS es el primer nivel territorial de la estructura organizativa del Servei Català de la Salut. De esta manera se acerca la gestión al ciudadano y se aumenta la eficiencia de la organización. Una muestra de este acercamiento a la comunidad es la participación de ésta, por primera vez, en la estructura directiva de la RS. La LOSC reserva al SS el papel de órgano de descentralización de la estructura organizativa del SCS. La Regió Sanitària Barcelonès Nord i Maresme está formada por dos sectores sanitarios: Barcelonès Nord y El Maresme.

El gerente de la RS asume la dirección y la gestión. Es el responsable de ejecutar las políticas propuestas desde la dirección del SCS, y también los acuerdos adoptados por el Consejo de Dirección de la Región. Uno de los resultados del proceso de despliegue territorial del SCS fue la determinación de las funciones básicas adscritas a las RS, según los diferentes ámbitos de responsabilidad y de los recursos humanos de la región, y que son las siguientes, ya descritas anteriormente: Planificación; Compra de servicios; Atención al cliente; Control de gestión y Soporte operativo.

FIGURA 6.33. Organigrama de la RSBNM.

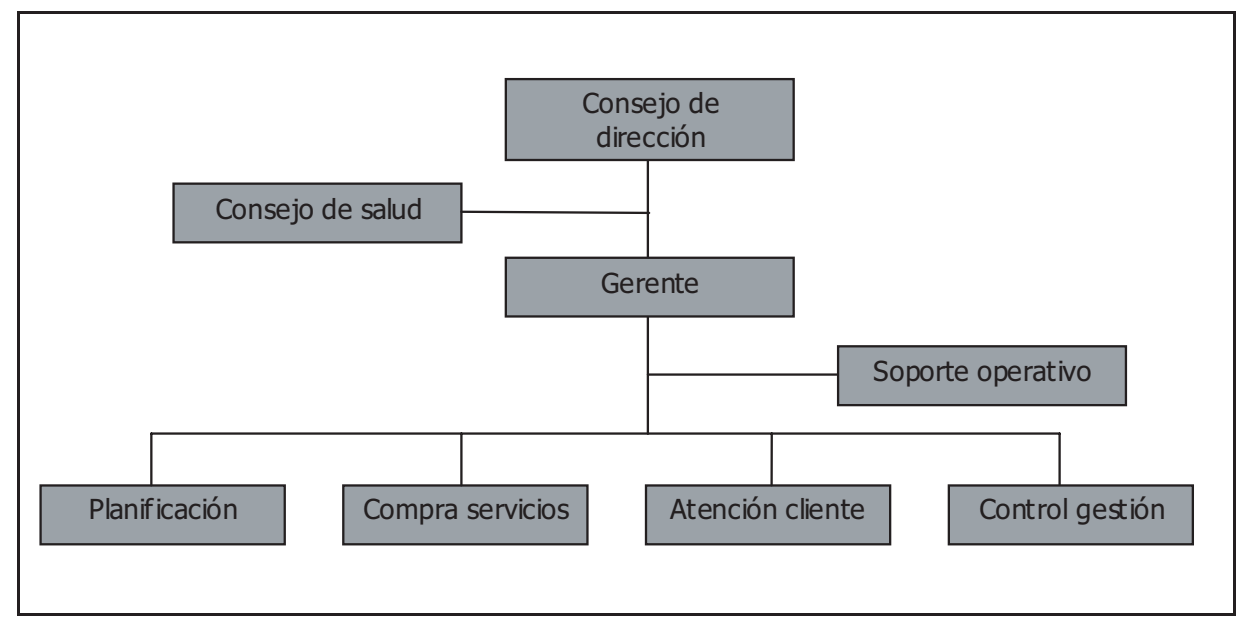

(C) Memòria d'activitat 1996 Regió Sanitària Barcelonès Nord i Maresme [1997, p. 51]. 


\subsection{REGIÓ SANITÀRIA CENTRE}

\subsubsection{DESCRIPCIÓN, TERRITORIO Y POBLACIÓN}

La Regió Sanitària Centre — RSC— comprende básicamente las comarcas del Solsonès, la Cerdanya, el Berguedà, el Bages, Osona, el Vallès Oriental y el Vallès Occidental. Según los datos del censo de 1991, la RSC tiene un total de 1.243.508 habitantes y una extensión de $6.577 \mathrm{~km}^{2}$. Actualmente constituye el 20,5\% de la población total de Catalunya y es la segunda región en número de habitantes. La densidad de población de la RSC es de 189 habitantes por $\mathrm{km}^{2}-187,5$ en Catalunya - y la distribución entre las diferentes comarcas y sectores es muy variada - los sectores sanitarios Bages, Berguedà, Cerdanya y Solsonès son los que tienen una densidad de población menor por causa de las comarcas de los Pirineos que los integran, en el otro extremo se sitúa el Sector Sanitari Sabadell con una densidad de 1.418 habitantes por $\mathrm{km}^{2}$, formado básicamente por poblaciones urbanas-.

TABLA 6.36. Datos sociodemográficos de la RSC por SS.

\begin{tabular}{|l|r|r|r|r|r|}
\hline \multicolumn{2}{|c|}{ Sabadell } & $\begin{array}{r}\text { Terrassa, Rubíy } \\
\text { Sant Cugat }\end{array}$ & $\begin{array}{r}\text { Osona y } \\
\text { Vallès Oriental }\end{array}$ & $\begin{array}{r}\text { Bages, Berguedà, } \\
\text { Cerdanya y Solsonès }\end{array}$ & Total \\
\hline Habitantes & 385.815 & 262.698 & 377.786 & 217.209 & $\mathbf{1 . 2 4 3 . 5 0 8}$ \\
\hline 0-14 años & 77.953 & 52.445 & 74.292 & 36.269 & $\mathbf{2 4 0 . 9 5 9}$ \\
\hline 15-64 años & 266.577 & 179.235 & 257.015 & 142.892 & $\mathbf{8 4 5 . 7 1 9}$ \\
\hline$>65$ años & 41.285 & 31.018 & 46.479 & 38.048 & $\mathbf{1 5 6 . 8 3 0}$ \\
\hline $\begin{array}{l}\text { Indice de } \\
\text { dependencia }\end{array}$ & 44,73 & 46,57 & 46,99 & 52,01 & $\mathbf{4 7 , 0 4}$ \\
\hline $\begin{array}{l}\text { Indice de } \\
\text { envejecimiento }\end{array}$ & 52,96 & 59,14 & 62,56 & 104,90 & $\mathbf{6 5 , 0 9}$ \\
\hline Densidad & 2.189 & 1.056 & 179 & 55 & $\mathbf{1 8 9}$ \\
\hline $\begin{array}{l}\text { Crecimiento } \\
\text { 1986-1991 }\end{array}$ & 1,29 & 1,53 & 1,41 & 0,13 & $\mathbf{1 , 1 6}$ \\
\hline Natalidad & 9,56 & 10,70 & 10,38 & 7,98 & $\mathbf{9 , 7 8}$ \\
\hline Mortalidad & 6,65 & 7,06 & 7,24 & 10,01 & $\mathbf{7 , 5 0}$ \\
\hline
\end{tabular}

(C) Memòria d'activitat 1996 de la Regió Sanitària Centre [1997, p. 27].

Las poblaciones de los sectores que integran la RSC muestran diferencias muy notables, los sectores que incluyen las comarcas rurales del Solsonès, la Cerdanya, el Bages, el Berguedà y Osona son las que presentan una población más envejecida. Los sectores que comprenden las comarcas del Vallès Oriental y del Vallès Occidental son los que presenten las poblaciones más jóvenes. La RSC 
tiene un índice de envejecimiento del 65\%, donde destacan los sectores Bages, Berguedà, Cerdanya y Solsonès con un índice mayor, y el Sector Sanitari Sabadell con índice menor.

El índice de dependencia global de la población de la RSC es del 47\%. Los sectores que se diferencian más son los del Bages, Berguedà, Cerdanya y Solsonès con un $52 \%$, ya que su población está más envejecida.

El crecimiento de la población de la RSC durante el período 1986-1991 ha sido bajo. La tasa de crecimiento anual, condicionada por la natalidad, mortalidad y movimientos migratorios, ha sido del 1,16\% durante estos años. La tasa de natalidad de la RSC en el 1994 es de 9,78 nacidos vivos por 1.000 habitantes, lo que supone una disminución respecto a la de los años anteriores. La distribución entre los sectores se modifica respecto de los años anteriores, ya que el Sector Sanitari Sabadell, con una población más joven, presenta una natalidad más baja que Osona y el Vallès Oriental. Los sectores sanitarios Bages, Berguedà, Cerdanya y Solsonès presentan la tasa de natalidad más baja.

La tasa bruta de mortalidad de la RSC, obtenida a partir del período 1989-1993, es de 7,50 muertos por 1.000 habitantes, inferior a la de Catalunya, que es de 8,53. Los sectores formados por comarcas rurales son los que presentan unas tasas superiores, por causa del envejecimiento de la población.

El crecimiento vegetativo es positivo en el conjunto de la RSC. Solamente los sectores Bages, Berguedà, Cerdanya y Solsonès tienen un crecimiento vegetativo negativo. La pirámide de edades de la RSC refleja claramente su estructura. La fuerte bajada de la natalidad en estos últimos años produce una disminución importante de la base de la pirámide. 


\subsubsection{SECTORES SANITARIOS Y ABS}

La RSC sufrió una modificación en su delimitación territorial de acuerdo con el Decreto 114/1994, de 3 de mayo, de delimitación de las RS y de los SS del SCS. Se han incorporado las ABS de la Cerdanya y del Solsonès, las cuales pertenecían a la RSG y a la RSL, respectivamente.

FIGURA 6.34. Mapa de la RSC.

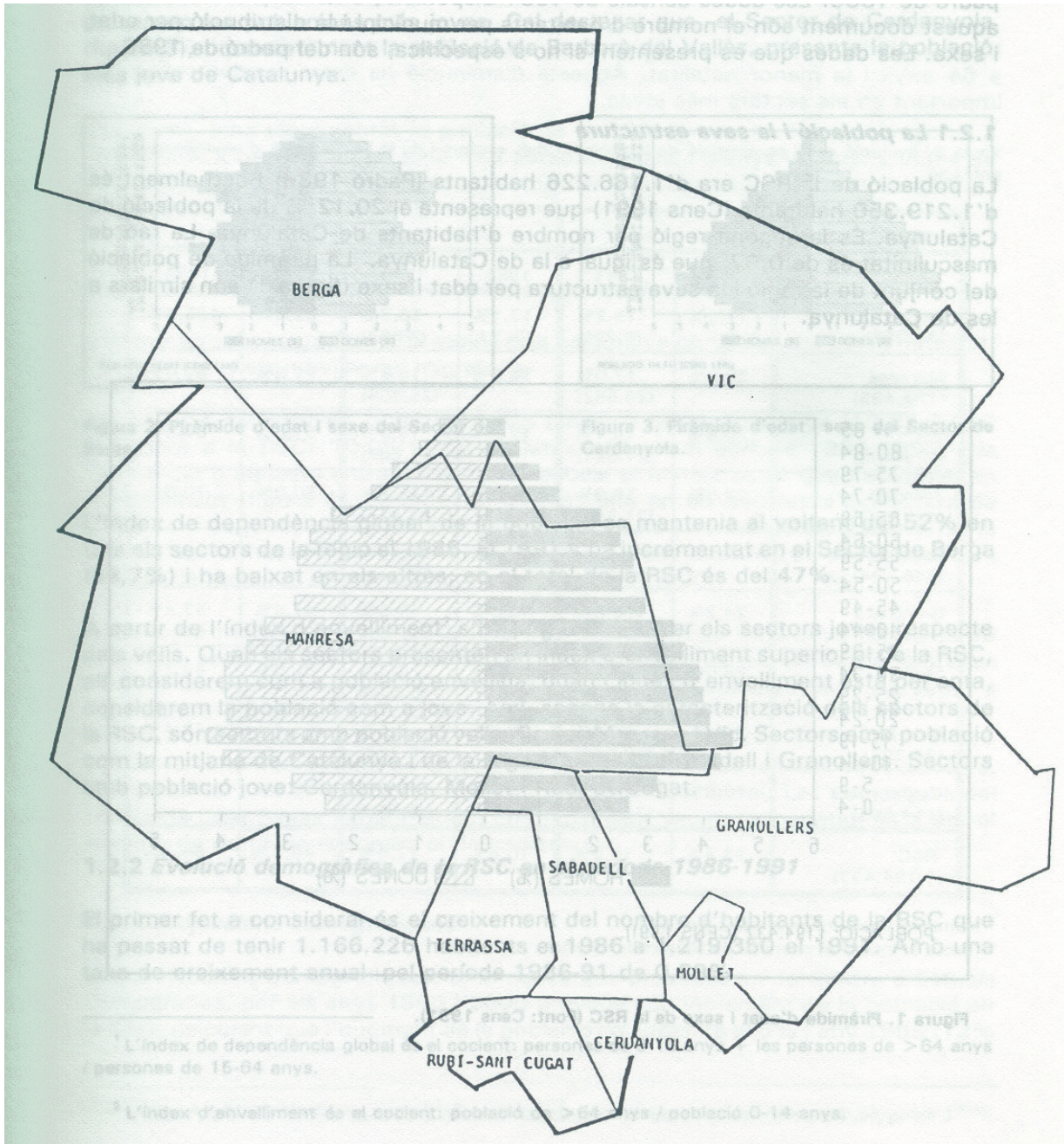

(C) Pla de Salut de la Regió Sanitària Centre 1993_-1995 [1993, p. 15]. 


\subsubsection{SS BAGES, BERGADÀ, CERDANYA Y SOLSONÈS}

Este sector está compuesto por las comarcas del Bages, el Bergadà, la Cerdanya y el Solsonès, con un total de 100 municipios y 19 ABS.

TABLA 6.37. ABS de los SS Bages, Bergadà, Cerdanya y Solsonès.

\begin{tabular}{|l|l|}
\hline Comarcas & ABS \\
\hline Bages & $\begin{array}{l}\text { Artés, Cardona, Montserrat, Manresa 1, Manresa 2, Manresa 3, Manresa 4, } \\
\text { Moià, Navàs-Balsareny, Sallent, Navarcles-Sant Fruitos de Bages, } \\
\text { Sant Joan de Vilatorrada, Sant Vicenç de Castellet, Súria. }\end{array}$ \\
\hline Bergadà & Bergadà Centre, Baix Bergadà, At Bergadà. \\
\hline Solsonès & Solsonès. \\
\hline Cerdanya & Cerdanya. \\
\hline
\end{tabular}

Fuente: Elaboración propia a partir de la Memòria d'activitat 1996 de la Regió Sanitària Centre [1997].

\subsubsection{SS OSONA Y VALLÈS ORIENTAL}

Este sector está formado por las comarcas de Osona y del Vallès Oriental, con un total de 92 municipios y 23 ABS.

TABLA 6.38. ABS de los SS Osona y Vallès Oriental.

\begin{tabular}{|l|l|}
\hline Comarcas & ABS \\
\hline Vallès Oriental & $\begin{array}{l}\text { Caldes de Montbui, Cardedeu, La Garriga, Montornès-Montmeló, } \\
\text { Sant Celoni, Granollers 1, Granollers 2, Granollers 3, Granollers 4, } \\
\text { Vall del Tenes, Martorelles, Parets del Vallès, La Llagosta, Mollet del Vallès. }\end{array}$ \\
\hline Osona & $\begin{array}{l}\text { Centelles, Manlleu, Lluçanès, Sant Hipòlit de Voltregà, Sant Quirze de Besora, } \\
\text { Santa Eugènia de Berga, Tona, La Vall del Ges, Vic. }\end{array}$ \\
\hline
\end{tabular}

Fuente: Elaboración propia a partir de la Memòria d'activitat 1996 de la Regió Sanitària Centre [1997].

\subsubsection{SS TERRASSA, RUBÍ Y SANT CUGAT}

El sector se sitúa dentro de la comarca del Vallès Occidental y está formada por 7 municipios y 9 ABS.

TABLA 6.39. ABS de los SS Terrassa, Rubí y Sant Cugat.

\begin{tabular}{|l|l|}
\hline Comarcas & ABS \\
\hline Vallès Occidental & $\begin{array}{l}\text { Terrassa A, Terrassa B, Terrassa C, Terrassa D, Terrassa E, Terrassa F, } \\
\text { Rubí 1, Rubí 2, Sant Cugat. }\end{array}$ \\
\hline
\end{tabular}

Fuente: Elaboración propia a partir de la Memòria d’activitat 1996 de la Regió Sanitària Centre [1997]. 


\subsubsection{SS SABADELL}

Este Sector también está situado dentro de la comarca del Vallès Occidental y está formado por 13 municipios y 18 ABS.

TABLA 6.40. ABS de los SS Sabadell.

\begin{tabular}{|l|l|}
\hline Comarcas & ABS \\
\hline Vallès & Castellar del Vallès, Sabadell 1-A, Sabadell 1-B, Sabadell 2, Sabadell 3-A, \\
Occidental & Sabadell 3-B, Sabadell 4-A, Sabadell 4-B, Sabadell 5, Sabadell 6, Sabadell \\
& $\begin{array}{l}\text { 7, Santa Perpètua de Mogoda, Cerdanyola 1, Cerdanyola 2, Ripollet, } \\
\text { Barberà del Vallès, Cuitat Badia, Montcada i Reixac. }\end{array}$ \\
\hline
\end{tabular}

Fuente: Elaboración propia a partir de la Memòria d'activitat 1996 de la Regió Sanitària Centre [1997].

\subsubsection{ESTRUCTURA CORPORATIVA}

Durante el año de 1996 y por causa de la pérdida de efectivos humanos, por motivos diversos, pero básicamente por el ajuste presupuestario y de plantillas se tuvo que reorientar el modo de funcionamiento de la RSC.

Con relación a los SS, continúan sus trabajos en el territorio aprovechando su proximidad con los proveedores y los ciudadanos y del conocimiento del entorno, pero a la vez, buscando la máxima coordinación con los servicios centrales de la RSC.

Una gran parte de los problemas del día a día se pueden resolver dentro del SS, que ha de proporcionar respuestas a los problemas que se presentan y al mismo tiempo planificar y estudiar las líneas de futuro junto con los servicios centrales de la región, bajo coordinación y supervisión de la gerencia. 
FIGURA 6.35. Organigrama de la RSC.

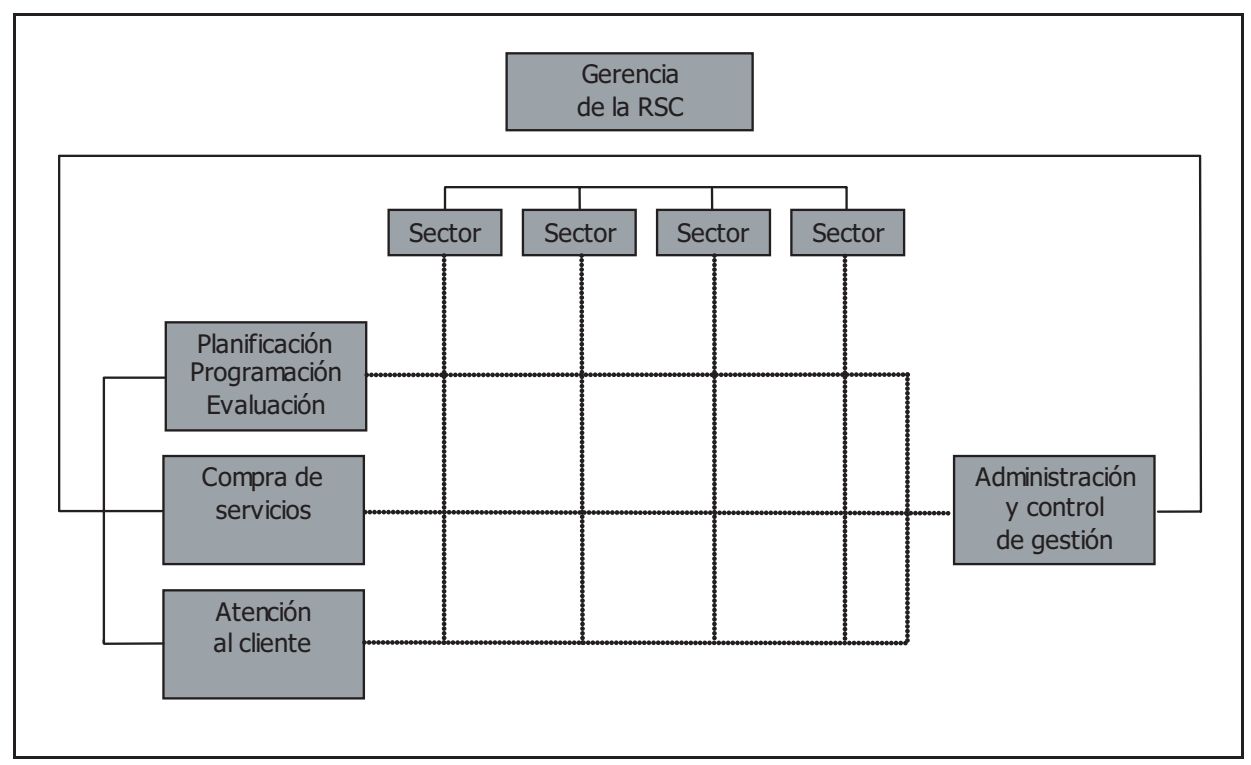

(C) Memòria d'activitat 1996 de la Regió Sanitària Centre [1997, p. 44].

\subsection{REGIÓ SANITÀRIA BARCELONA CIUTAT}

\subsubsection{DESCRIPCIÓN, TERRITORIO Y POBLACIÓN}

Barcelona concentra entorno del 30\% de la población de Catalunya. Ubicada en una superficie de $99 \mathrm{~km}^{2}$, presenta una población de 1.508 .805 habitantes, según datos del padrón municipal de 1997. Eso supone una densidad de población aproximadamente de 15.230 habitantes por $\mathrm{km}^{2}$. Las características más destacables en los cambios demográficos de Barcelona en los últimos años son la tendencia al decrecimiento de la población — del año 1981 al 1996 se observa una disminución de más de 100.000 habitantes_, como también la tendencia al envejecimiento, ya que el número de personas mayores de 65 años supera el número de jóvenes menores de 15 años. 
TABLA 6.41. Datos sociodemográficos de Barcelona.

\begin{tabular}{|l|r|l|}
\hline Datos estructurales & 1.508 .805 & Número de habitantes \\
\hline Población & 99,07 & Km $^{2}$ \\
\hline Extensión & 15.230 & Habitantes por Km² \\
\hline Densidad de población & 1 & Número de comarcas \\
\hline División territorial & 1.403 .265 & PTA por habitante [renta disponible] \\
\hline Renta per cápita & $73,5-81,5$ & Años para hombre-mujer \\
\hline Esperanza de vida & 10,5 & Defunciones por 1.000 habitantes \\
\hline Tasa de mortalidad & 7,9 & Nacimientos por 1.000 habitantes \\
\hline Tasa de natalidad &
\end{tabular}

(C) Consorci Sanitari de Barcelona. Recull de dades 1997 [1997, p. 2].

TABLA 6.42. Pirámide de edades.

\begin{tabular}{|l|r|r|r|}
\hline Años & Hombres & Mujeres & Total \\
\hline$<15$ & 121.726 & 115.102 & 236.828 \\
\hline $15-29$ & 193.718 & 186.946 & 380.664 \\
\hline $30-44$ & 158.996 & 169.558 & 328.554 \\
\hline $45-64$ & 193.872 & 218.143 & 412.015 \\
\hline$>65$ & 107.676 & 177.805 & 285.481 \\
\hline Total & $\mathbf{7 7 5 . 9 8 8}$ & $\mathbf{8 6 7 . 5 5 4}$ & $\mathbf{1 . 6 4 3 . 5 4 2}$ \\
\hline
\end{tabular}

(C) Memòria d'activitat 1996 Regió Sanitària Barcelona Ciutat [1997, p. 24]. 


\subsubsection{SECTORES SANITARIOS Y ABS}

La RSBC está dividida en 10 SS, que son los siguientes:

- Ciutat Vella.

- Eixample.

- Sants-Montjuïc.

- Les Corts.

- Sarrià-Sant Gervasi.

- Gràcia.

- Horta-Guinardó.

- Nou Barris.

- Sant Andreu.

- Sant Martí.

TABLA 6.43. Sectores sanitarios y ABS de la RSBC.

\begin{tabular}{|l|l|}
\hline SS & ABS \\
\hline Ciutat Vella & 1A, 1B, 1C, 1D, 1E. \\
\hline Eixample & 2A, 2B, 2C, 2D, 2E, 2G, 2H, 2I, 2J, 2K. \\
\hline Sants-Montjuïc & $3 \mathrm{~A}, 3 \mathrm{~B}, 3 \mathrm{C}, 3 \mathrm{D}, 3 \mathrm{E}, 3 \mathrm{~F}, 3 \mathrm{G}$. \\
\hline Les Corts & $4 \mathrm{~A}, 4 \mathrm{~B}, 4 \mathrm{C}$. \\
\hline Sarrià-Sant Gervasi & $5 \mathrm{~A}, 5 \mathrm{~B}, 5 \mathrm{C}, 5 \mathrm{D}, 5 \mathrm{E}$. \\
\hline Gràcia & $6 \mathrm{~A}, 6 \mathrm{~B}, 6 \mathrm{C}, 6 \mathrm{D}$. \\
\hline Horta-Guinardó & $7 \mathrm{~A}, 7 \mathrm{~B}, 7 \mathrm{C}, 7 \mathrm{D}, 7 \mathrm{E}, 7 \mathrm{~F}, 7 \mathrm{G}$. \\
\hline Nou Barris & $8 \mathrm{~A}, 8 \mathrm{~B}, 8 \mathrm{C}, 8 \mathrm{D}, 8 \mathrm{E}, 8 \mathrm{~F}, 8 \mathrm{G}, 8 \mathrm{H}, 8 \mathrm{I}$. \\
\hline Sant Andreu & 9A, 9C, 9D, 9E, 9F, 9G. \\
\hline Sant Martí & $10 \mathrm{~A}, 10 \mathrm{~B}, 10 \mathrm{C}, 10 \mathrm{D}, 10 \mathrm{G}, 10 \mathrm{H}, 10 \mathrm{I}, 10 \mathrm{~J}$. \\
\hline
\end{tabular}

Fuente: Elaboración propia a partir de la Memòria d'activitat 1996 Regió Sanitària Barcelona Ciutat [1997]. 
FIGURA 6.36. Mapa de la RSBC.

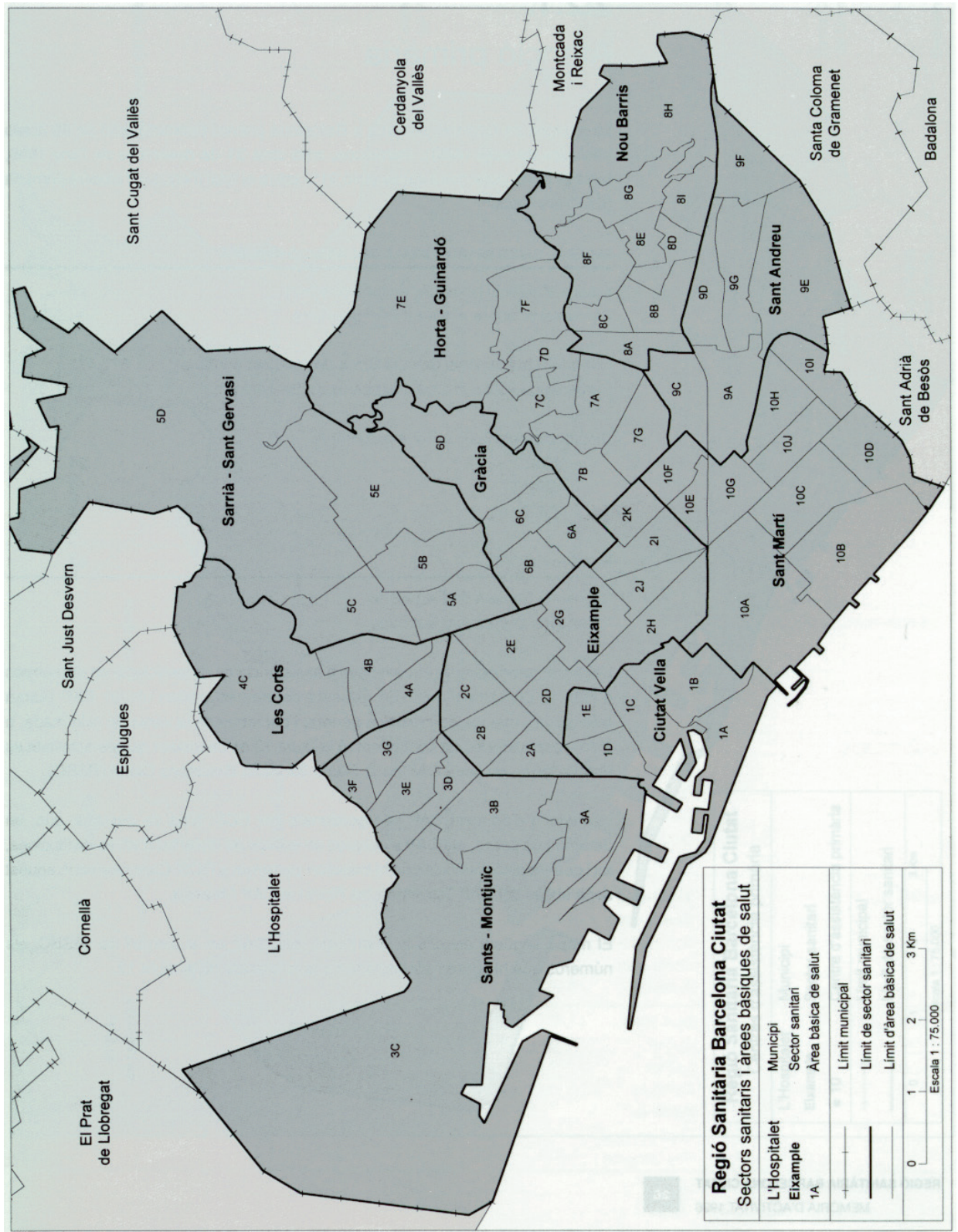

(C) Memòria d'activitat 1996 Regió Sanitària Barcelona Ciutat [1997, p. 25]. 


\subsubsection{ESTRUCTURA CORPORATIVA}

En la ciudad de Barcelona desde el mes de marzo de 1997, el Consorci Sanitari de Barcelona, ente público constituido por la Generalitat de Catalunya y el Ayuntamiento de Barcelona, adscrito al Servei Català de la Salut asume las funciones de dirección y coordinación de la gestión de los centros, los servicios y los establecimientos de protección de la salud y de atención sanitaria y sociosanitaria en el ámbito de la Regió Sanitària Barcelona Ciutat y se estructura en los órganos que determinan sus estatutos. La Junta General es el órgano de gobierno superior del Consorci y ejerce las funciones que el artículo 26 de la Ley 15/1990, de 9 de julio, y las disposiciones concordatarias, atribuyen al Consejo de Dirección de las Regiones Sanitarias, en el ámbito de la ciudad de Barcelona.

FIGURA 6.37. Organigrama de la RSBC.

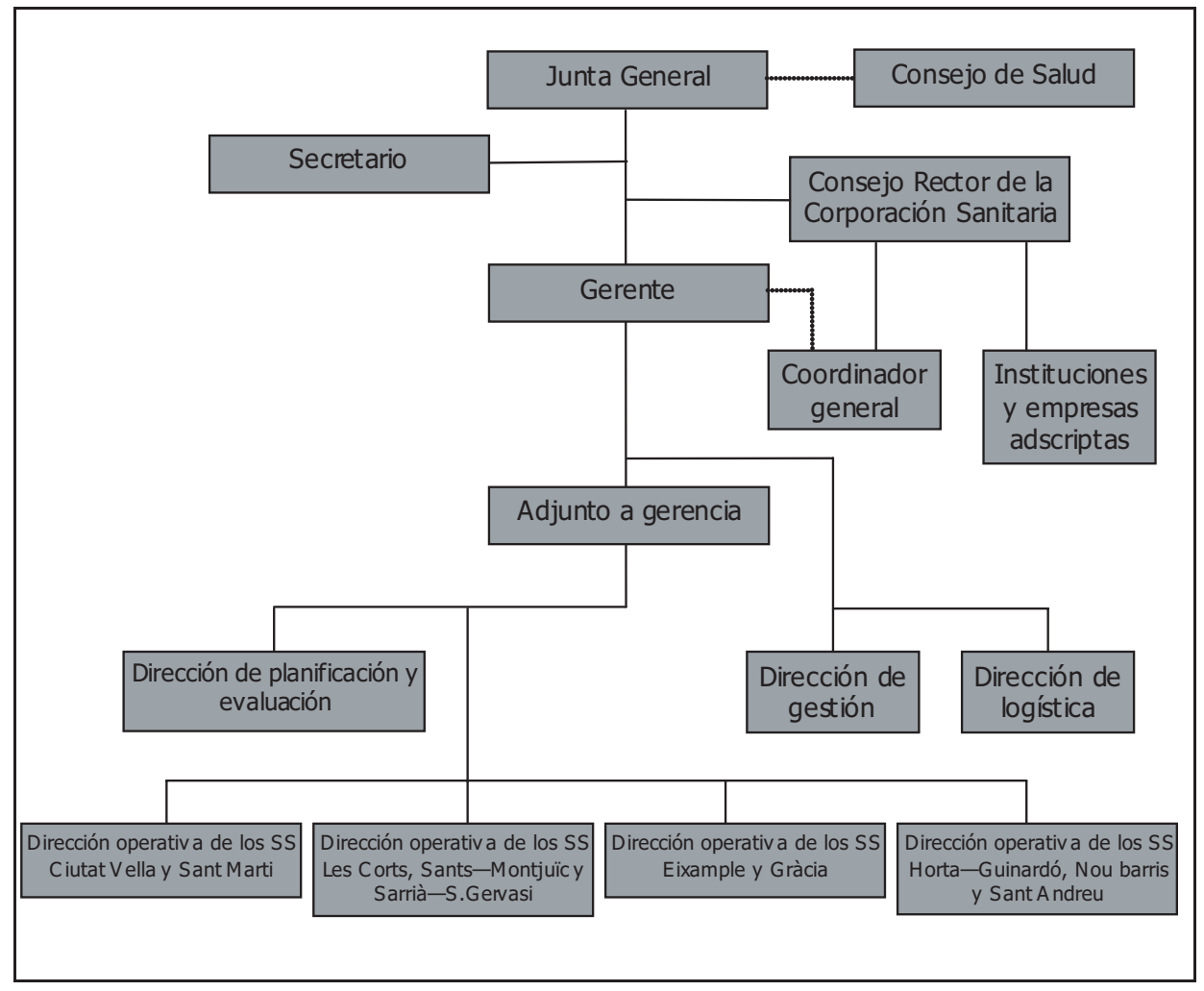

(C) Memòria d'activitat 1997 Regió Sanitària Barcelona Ciutat [1998, p. 19]. 


\subsection{HOSPITALES DE ALTA TECNOLOGÍA}

\subsubsection{HOSPITAL DE LA SANTA CREU I SANT PAU}

\subsubsection{ANTECEDENTES HISTÓRICOS}

Durante los primeros años del siglo XXI, y con la vista puesta en un nuevo milenio, abrió las puertas un nuevo Hospital de la Santa Creu i Sant Pau. Fue la tercera construcción de nueva planta destinada a recoger el testimonio de la continuidad asistencial más antigua de Europa. El futuro hospital se emplazó en el mismo recinto del actual. El proyecto previó acoger alrededor de unas setecientas camas, proporcionando gran importancia al desarrollo de la actividad ambulatoria, de acuerdo con las previsiones futuras de utilización de los hospitales. En cinco niveles, el edificio se distribuye en tres naves separadas por partes interiores, que garantizaron la luminosidad. Las obras se iniciaron con la construcción de un aparcamiento con más de mil plazas. La misión del nuevo edificio fue colaborar a superar el alto nivel asistencial de la institución, un propósito que ha marcado los dos grandes momentos históricos del hospital: La entrada del siglo XX, con la adopción del conjunto arquitectónico proyectado por Lluís Domènech i Montaner — para ejecutar la voluntad testamentaria del banquero Pau Gil i Serra- y, la entrada del siglo XV, con la construcción del Hospital de la Santa Creu, como consecuencia del acuerdo de los dirigentes municipales y eclesiásticos de Barcelona para fusionar las deficientes casas de enfermos existentes y dar lugar a un sólido proyecto de hospital general que, durante quinientos años — hasta la obertura del Clínic, en 1907_, fue el único de la ciudad. Pero se tiene que hacer un paso atrás. La historia hospitalaria de Barcelona se puede remontar probablemente hasta el siglo IX, con un Hospital o Casa de Mesells, dedicado a Santa Margarida, para leprosos, situado fuera de la ciudad y dirigido seguramente por una orden mixta formada por clérigos y laicos.

Con todo, es a partir del cambio de milenio que se puede recomponer el hilo histórico con claridad. En el año 985, Barcelona es destruida por las tropas de al-Mansür. Superados los temores militaristas, la reconstrucción de la ciudad enlaza con un período de recuperación económica. Se construye la catedral románica y, a su lado, en la bajada de la Canonja, se encuentra el Hospital de Guitart, una pequeña casa que este barcelonés dona al hospital. Durante el siglo 
XI recibe propiedades, fincas, legados y donaciones para su mantenimiento. Entre los donantes está el obispo Deodat y el conde Ramon Berenguer I y su esposa Elisabet. Fue conocido también como Hospital de la Canonja, como Casa de Hospital de la Seu o como de la Santa Creu i Santa Eulàlia. En el año 1218, cuando se crea la «Orde de la Mercè», el Rey Jaume I la concede a este hospital.

En medio del siglo XII otro ciudadano vinculado a Ramon Berenguer IV, es el origen del Hospital de Marcús, situado en la plaza que todavía tiene el nombre de Bernat Marcús y donde está la capilla de este hospital, que se mantuvo con la llegada del fundador y con donaciones de barceloneses. Durante el siglo XIV vive tiempos difíciles. En 1335, una orden del Vaticano concedía indulgencias a aquellos que la socorriesen. Finalmente, el municipio se hace cargo. Los consejeros de la ciudad también regentaron y administraron el Hospital del Almoina, o de Santa Marta, fundado en 1308 por el ciudadano Pere Desvilar en el suburbio llamado de Vilanova. Disponía de buenas rentas y hacia diligencias periódicas. Por otro lado, Pere Vilar, canónigo de la Catedral, fundó en el raval — y que después fue el Monasterio de los Jerónimos - un pequeño albergue que acogía muy pocos enfermos. Su principal servicio era la maternidad, acogía niños abandonados en las buhardillas hasta que les encontraba una nodriza. Era conocido como Hospital del Pere Vilar o de Sant Macià. Había, todavía, dos casas más: Un hospital poco documentado al lado del Monestir d'Agustins de Santa Eulàlia del Camp, y el Hospital de Colom — que sucede el Hospital de Guitart-, que es el precedente físico directo del Hospital de la Santa Creu.

El canónigo Joan Colom, que fue tesorero de Jaume I, construye el hospital donde ya había unas casas de enfermos dispersas. Dótalo de rentas en vida y al morir en el año 1229 la institución queda bajo la administración del obispo y del capitolio de la catedral. En el año 1236 se dictan las primeras ordenaciones para el hospital que fueron encontradas. La situación al final del siglo XIV, la definen una asistencia y unas instalaciones de pésima calidad, y hasta el año 1401 no llega la solución: El 1 de febrero, el Consell de Cent —el gobierno de la ciudad - aprueba una moción para intentar reunir los hospitales en uno sólo. El 15 de marzo, en la Sala del Trentenari y en el Palau Episcopal se celebran dos reuniones simultáneas: Por un lado, los consejeros, y por otro, el obispo y el capitolio. Se consigue un acuerdo: Constituir el Hospital o Casa de la Santa Creu, con cuatro de los seis hospitales, y hacerlo en los terrenos de la antigua casa de Colón, en la calle que hoy se conoce como Carrer de l'Hospital. En aquel verano, el capitolio es incorporado en el Hospital de Santa Margarida, y el Prior de Santa Eulàlia del Camp también se agrega a él. En el 5 de 
septiembre, el Papa Benet XIII —el Papa Luna— firma un documento en Avinyó aprobando la fusión.

La gestión de la institución queda encomendada a dos canónigos y a dos prohombres, que son renovados, por mitad a cada dos años, en el día de la Santa Creu, en el mes de mayo. Este es el origen de la Molt Il.lustre Administració - MIA — , que perdura hasta hoy, con un único cambio substancial por la incorporación de la Generalitat en 1990 en las mismas condiciones que el Ayuntamiento y la Iglesia. Volviendo al 1401, en el 13 de febrero se inician los trabajos de construcción y en el 17 de abril se colocan las primeras cuatro piedras, con la presencia del Rey Martí I, la Reina Maria de Luna, Jaume de Prades —en representación del príncipe Martí el Jove, de Sicília—, el obispo Joan Ermengol y los consejeros. En una primera fase, las obras se prolongan hasta el 1450. Durante los siglos XVI y XVII se construyen la nave de poniente, las dos escaleras monumentales y el gran alambrado de hierro de forja que valla el patio a medio día.

Arquitectónicamente, el Hospital de la Santa Creu sigue una estructura similar a la de los palacios señoriales medievales catalanes, que definen un perímetro cuadrado con cuatro naves y un patio central, como será también el Hospital de Santa Maria de Lleida.

Las técnicas constructoras son las propias de la arquitectura civil gótica de Catalunya — los arcos, las vueltas de cruce- como el Saló del Tinell, las Drassanes, el Saló de Llotja o la Capella de Santa Agata. El edificio se encargó a Guillem Abiell, maestre de obras de las iglesias del Pi, del Carme, de Montsió y de Sant Jaume. El sustento del hospital proviene, de los privilegios que le otorgan reyes y papas, como la autorización de hacer colectas por las iglesias en los días festivos, el derecho a postular, indulgencias, protección real, franquicias, el derecho a recibir los bienes de los muertos intestados en el hospital sin descendencia legítima, o poder colectar a toque de campana, privilegio que se usó hasta los inicios del siglo XIX unos recaudadores conocidos como «baciners de la Creu».

El Rey Felipe II, en el año 1587, concede un nuevo privilegio a la Santa Creu como confirmación de una autorización militar anterior [1579]: Los administradores del hospital decidirán donde han de hacer sus representaciones los comediantes de paso por la ciudad, una vez aprobadas las piezas por el obispo o por la Inquisición. De esta manera, con el que pagaban a la compañía los asistentes, se obtenían recursos. En el año 1596 se contrata la construcción de la Casa de las Comèdies en los terrenos de una casa y de un huerto delante de la 
puerta de los Ollers, actual «Carrer de los Escudellers». —Durante el siglo XVIII, el teatro se amplía y tiene que ser reconstruido por causa de un incendio, hecho que se repite en 1915, tres años antes de ser vendido-.

Durante el siglo XVII, se destacan los trabajos de construcción. Por un lado, en 1638 la nave de poniente es destruida por un gran incendio, pero se vuelve a levantar en el año siguiente gracias a la colaboración de los barceloneses. Por otro, el 25 de marzo de 1629 se inicia la edificación de la Casa de Convalescència — en la actualidad, sede de la Biblioteca de Catalunya - con el legado que Lucrècia de Gualba, señora de la Batllòria, destinó a las obras de piedad. El incendio de 1638 y el cerco de la ciudad entre 1651 y 1652 lentizan los trabajos, pero nuevos legados hacen que la obra quede terminada en 1680. —La Casa de Convalescència fue conocida también como Hospital de Sant Pau, en honor de Pau Ferran, que hizo uno de los legados-.

Por causa del Decreto de Nueva Planta, en 1716, con el traslado de la universidad a Cervera, el hospital recibe el castigo del ayuntamiento, de la actividad docente — este hecho a las horas tendría su aspecto positivo por la desvinculación de la rigidez de la enseñanza oficial—.

Cuando en 1764, impulsado por Pere Virgili, se inaugura el Reial Col.legi de Cirurgia al lado de la Casa de Convalescència, el Hospital de la Santa Creu entra en el camino de la docencia práctica, de la incorporación del saber médico europeo y de la equidad entre Medicina y Cirugía, en una tradición en que los cirujanos eran considerados poco más que barberos. Los profesores del Colegio eran, por tanto, cirujanos de un hospital por donde pasaban unos cuatro mil enfermos al año, aunque solamente se atendiesen los casos quirúrgicos. La asistencia a los enfermos la hacían normalmente hombres y mujeres voluntarios, pero con la lógica discontinuidad.

En el año 1784 Jaume Sayols, un vendedor de ropa, con nuevos compañeros, se ofrece a cuidar de los enfermos haciendo vida de pobreza en comunidad. En 1791 reciben la constitución como Pia Societat de Hermanos de la Caritat de la Santa Creu. A principio del siglo XIX, después de un intento que no cuajó de unas monjas Paulas Francesas, surge una Congregació de Germanes Hospitalàries.

Con la ocupación napoleónica [1808-1814], el hospital acogió los soldados franceses heridos más los enfermos de la ciudad, situación que se complicó en 1809 con una epidemia de varicela. En la segunda mitad del siglo, tres epidemias de cólera y una de fiebre amarilla obligaron a atender los enfermos en espacios habilitados como el Seminari, la Casa de Convalescència o el Convent de las Penedides. 
Desde el año 1401 hasta el final del siglo XIX, la población de Barcelona se había multiplicado por quince, pasando de los treinta y cinco mil habitantes a los más de quinientos treinta mil. El hospital se había quedado pequeño y se había envejecido. En el invierno, los enfermos se helaban en las inmensas naves góticas y, en el verano, el olor de cuatro hileras de camas era insoportable. Esta penuria, no obstante, contrastaba con un momento de gran inquietud innovadora entre el cuerpo facultativo. La facultad de Medicina había vuelto a Barcelona en 1843, y en el entorno de la facultad y del hospital se originó una generación dispuesta a construir una Medicina científica. En un clima de regeneracionismo cultural y político, estos médicos viven la celebración en Barcelona del Congreso Médico de 1888. Son los doctores Giné, y Partagàs, los introductores de la Dermatología, la Psiquiatría, la Farmacología y la Antisepsía; Bartomeu Robert, defensor de la culturalización del médico; Jaume Pi i Sunyer, seguidor de las tesis de Claude Bernard, y Miquel Angel Fargas, creador de la escuela ginecológica catalana.

La evolución de esta generación había de vivir un punto de inflexión histórica, por dos factores determinantes. El primero: La apertura del Hospital Clínic, en 1906 se traslada la facultad de Medicina, y el segundo: El inicio de la construcción, en 1902, en unos terrenos al pie del Guinardó, de un centro para el cual se habían proyectado 48 pabellones. Es el Hospital de Sant Pau. Pero, para asistir al nacimiento de este proyecto, se tiene que cambiar de escenario e ir hasta el mes de septiembre de 1892. Cuatro años antes de morir, el banquero Pau Gil i Serra, establecido en París, hace testamento y dispone que, una vez liquidada la banca familiar, se dedique la mitad de los dineros que queden para «la fundación de un Hospital Civil que con el nombre de San Pablo se construirá en la ciudad de Barcelona». El testamento tiene anexo un conjunto de instrucciones donde se precisa que el objetivo es el de acoger a los desgraciados, que el hospital se ha de construir de acuerdo con los mejores de París y que los dineros se han de destinar solamente a la construcción del edificio, y que después ha de ser cedido a quien preste la asistencia [La Xarxa Hospitalària d’Utilització Pública de Catalunya. Història d'una diversitat, 1994, p. 40].

Siguiendo otra de las disposiciones testamentarias, se convoca un concurso de proyectos. Dos tribunales han de decidir la adjudicación. Por un lado, un tribunal de arquitectos, en abril de 1900, escoge con reservas un proyecto de Josep Domènech i Estapà. Por otro, un tribunal de médicos desestima todos los proyectos presentados. El 19 de junio de 1901, los albaceas y la junta del Hospital de la Santa Creu, que han llegado a un acuerdo para hacer un plan general para los dos hospitales unidos, encargan un nuevo proyecto a Lluís 
Domènech i Montaner. Este hecho genera que Domènech i Estapà empiece, en el año siguiente, una polémica en los periódicos. Una comisión de abogados dictaminó que no existía la obligación de dar el premio si ninguno de los proyectos presentados reunía las condiciones necesarias. Además, para el acuerdo de los dos hospitales, se había cambiado de solar, de Sarrià a Can Xifré, el actual emplazamiento, cedido por su propietario al Hospital de la Santa Creu en el año 1889, y al lado del cual en el año siguiente el hospital había adquirido otros terrenos. Los albaceas de Pau Gil agregan más terrenos en el cambio con los de Sarrià.

El 15 de enero de 1902 se coloca la primera piedra. La obra, en una primera fase, se considera acabada en el año 1912. Los fondos del legado Gil se habían agotado. En 36.000 metros cuadrados, se incluía el Pabellón de la Administración [1905-1910], que permite la entrada al conjunto, dos pequeños pabellones de reconocimiento, seis pabellones de hospitalización y el edificio central de operaciones. En abril de 1913, el Hospital de la Santa Creu recibe los terrenos y los pabellones construidos, y adquiere el resto del terreno —en total, 145.470 metros cuadrados- Hoy, el Sant Pau está plenamente integrado en la trama Cerdà, respecto de la cual define una disposición en diagonal, por bien que en un principio se quiso un hospital «higiénico», situado «fuera de las aglomeraciones urbanas, pero no tan lejos que comprometiera su utilidad». Este fue uno de los motivos de escoger el actual emplazamiento, cerca de donde pasaba el tranvía eléctrico de Horta, y también por la leve inclinación del solar, que facilitaba los desagües.

Para la definición funcional del edificio se estudian centros hospitalarios de 131 ciudades diferentes Domènech i Montaner partió del modelo pabellonario de hospital, una tradición constructiva iniciada en la segunda mitad del siglo XVIII tomando como referencia los hospitales militares de campaña, y que abraza desde el Stone_-House de Plymouth [1756 — 1764] hasta el Johns Hopkins de Baltimore [1876 - 1889]. Sin duda, es el modelo en que pensaba Pau Gil cuando establecía que «las grandes escaleras, patios, galerías, para los convalecientes, laboratorios, capilla, etc., sean en todo conformes con las más bien realizadas en los hospitales de París» [La Xarxa Hospitalària d'Utilització Pública de Catalunya. Història d'una diversitat, 1994, p. 41], donde había muestras notables como el Lariboisière [1838 - 1854] o el nuevo Hôtel—Dieu [18661878]. La gran innovación de Domènech i Montaner fue aportar la solución de la concentración subterránea, emplazando en los subterráneos el entramado de servicios y comunicaciones, los pasillos entre bloques y las dependencias 
multitudinarias y de más uso. De esta manera, ofrecía una respuesta propia a la polémica entre hospital compacto y hospital pabellonario.

Estéticamente, el Hospital de la Santa Creu i Sant Pau es de una arquitectura de gran calidad y, como se ha dicho, una de las últimas propuestas sólidas amparadas en los supuestos creativos del Modernismo. El recurso a las vueltas catalanas de ladrillo plano para el tejado y cubiertas, y la ornamentación de estilo floral sobre cerámica de Valencia o vidriada y en mosaico, son algunos de los acentos de un estilo anteclásico. Lluís Domènech dirigió un equipo de arquitectos - entre los cuales había su hijo Pere, que le sucede una vez muerto, en el año 1923 - y artistas plásticos. Además del legado Gil —unos tres millones de pesetas en aquellos tiempos-, las obras de Sant Pau son posibles gracias a nuevas aportaciones particulares y a la adquisición del edificio de la Santa Creu por parte del Ayuntamiento, que lo compra por cinco millones de pesetas - la mitad de los cuales con suplemento de carácter benéfico- - y que instituye una subvención anual de 150.000 pesetas para colaborar en su mantenimiento. Con los dineros de la venta se construyen la iglesia y los dos edificios anexos. Es la generosidad de familias acomodadas lo que permite, entre 1914 y 1935, la construcción de otros nueve pabellones. También la participación popular ha colaborado, con actos como el celebrado en 29 de junio de 1928, en el que diez mil niños barceloneses acudieron con la recaudación de la «Festa de la Guardiola» en el solar que había de acoger el pabellón de Oncología. Allí, se lanzaron las huchas vacías en los fundamentos con valor de primera piedra. En el año siguiente, la captación recogió 300.000 pesetas que, junto con una donación anónima, servirán para comprar un gramo de radio y un aparato de Radioterapia. Mientras duraron las obras, la actividad continuó en el Hospital de la Santa Creu, que se había vendido en el año 1921, pero que no se había de entregar hasta que se completase el traslado. Aquella generación de médicos, articulada por vuelta del 1888, había evolucionado o dado paso a una generación neocentista, que matizaba el positivismo materialista.

Entre el Primero Congreso Universitario Catalán, celebrado en 1903, y la publicación de las conclusiones del Segundo Congreso en 1918, se consolida la separación entre el ensañamiento universitario - establecido en el Clínic — y la enseñanza libre, impartido sobretodo en las escuelas de especialidades del Santa Creu, con una larga tradición docente. Aquel nuevo Hospital de la Santa Creu i Sant Pau se forjaba, por tanto, con un espirito innovador, opuesto al inmovilismo oficial, conectado con los últimos conocimientos europeos. Es así como empieza el desglosamiento de ramas y la adopción de nuevas especialidades. Lluís 
Barraquer i Roviralta introduce la Neurología científica. Su hermano Josep Anton crea la escuela catalana de Oftalmología. Antoni Raventós i Avinyó impulsa la Neurocirugía. Enric Ribas i Ribas obtiene prestigio internacional con la Cirugía biliopancreática. Josep Maria Vilardell introduce el injerto óseo-periostio en la artrodesis.

Esta actividad innovadora contrastaba con una cierta lentitud en la hora de trasladarse, escalonadamente, a lo largo de trece años. El Dr. Torras i Pujalt, dos colaboradores y después un residente fueron los primeros en traer algunos enfermos, con la ayuda de las hermanas, en 30 de junio de 1916. En el año 1920, el doctor Joan Freixas i Freixas, un histórico de la casa y de la docencia práctica —el año 1886 ya impartía lecciones en la cabecera de los enfermos_-, ocupa con cuatro médicos más, el tercer pabellón habilitado. El 1925 se traslada Medicina infantil y, durante 1929, el resto de los servicios. El 16 de enero de 1930, fecha en que deja de funcionar el hospital viejo, el Rey Alfonso XIII inaugura el Hospital de la Santa Creu i Sant Pau. En aquellos momentos, cuenta con todas las especialidades médicas y quirúrgicas.

En el año 1932 se aprueba el Estatuto de Autonomía de Catalunya y en junio de 1933, en aplicación del artículo siete, se crea la Universitat Autònoma de Barcelona. El 26 de julio de 1936 la junta administrativa del Hospital acuerda el cambio de título antiguo de Hospital de la Santa Creu i Sant Pau por el de Hospital General de Catalunya. Después de la Guerra Civil, la economía del centro hospitalario se encontró fuertemente afectada y la Universitat de Barcelona fue reincorporada al conjunto de la enseñanza estatal española. En el año 1967 fue reconocida oficialmente la escuela del profesor Antoni Puigvert, que dirigía desde el año 1951 — y en octubre de 1968 se establece la Universitat Autònoma dentro del Hospital de la Santa Creu i Sant Pau-.

En el año 1955, los hermanos de la Pia Societat dejan de prestar servicio y son substituidos por las monjas. En esta época, se fue dejando de separar los enfermos por sexos y se empieza a agrupar por patologías, de manera que se deja de duplicar servicios. Hasta entonces, tal como se entra en el conjunto hospitalario, los pabellones de la derecha acogían hombres y los de la izquierda, mujeres. A lo largo de las décadas de los cincuenta y sesenta, se viven cambios en las especialidades. Se crean los servicios del Aparato respiratorio — que substituye Tisiología_, Broncología —que, fusionada con la anterior, forman la Broncopneumología-, Endocrinología, los departamentos de Regulación de líquidos y de Cirugía experimental, la unidad de Enfermedades coronarias y la Escuela de Cardio—angiología. 
El 30 de junio de 1965, la Molt Il.lustre Administració acuerda nombrar un director general para el hospital, hecho que se hace efectivo el 27 de enero de 1966. Este nombramiento, pionero en la época, representó un cambio en la filosofía de la Administración, que empezó a gestionar el centro de forma empresarial. A finales de los años sesenta se produce una transformación radical en el camino de la profesionalización de la asistencia, un hecho que afecta el cuerpo médico y también el de enfermería — las monjas fueron siendo substituidas por personal titulado- - Se firma el primer convenio con la Seguridad Social. Se incorporan nuevas dotaciones tecnológicas y se aplican avances científicos importantes. Se abre un importante servicio de Urgencias —octubre de 1967-. Con relación a la docencia, como se ha dicho, se recupera la vinculación con la reconstituida Universitat Autònoma de Barcelona [1968], se acogen tres cursos de pregraduación en Medicina con unos cuatrocientos estudiantes, ciento cincuenta residentes y unos ochenta becarios extranjeros de una veintena de países diferentes, se hacen cursos de postgrado y se acoge también la Escuela Universitaria de Enfermería.

En la actualidad, el centro se define dentro de la Red Hospitalaria de Utilización Pública -XHUP - como un hospital de nivel C —alta tecnología o de superespecialidades-, capaz de afrontar problemas de máxima complejidad con la más alta calidad asistencial. Sant Pau dispone de una gran experiencia en programas de trasplantes — se hizo el primer trasplante de médula ósea de España, en 1976, y el primer autotrasplante, en 1984 - Aquel mismo año, se hacia el primer trasplante de corazón del Estado. El programa de trasplante de fémur, para el tratamiento de los tumores óseos, trabaja activamente. De entre los implantes, destacan los intraoculares de cristal artificial, para las cataratas, el implante coclear, para ciertas sorderas, o la restauración de la mama amputada por cáncer. En cuanto a la Oncología, el hospital fue el primer a poner en marcha esta especialidad, con la cual colaboran todos los otros servicios y que cuenta con una notable dotación tecnológicaradioterapia, braquiterapia, acelerador lineal y bombas de cobalto- - Otras áreas de excelencia del centro son la cirugía de alta especialización, la incorporación de las técnicas diagnósticas más sofisticadas o los programas obstétricos —embarazo de alto riesgo, fecundación in vitro y diagnóstico preembarazo de enfermedades genéticas- Con relación a la investigación, se desarrollan numerosas líneas de investigación-Biomecánica, Neuropsicofarmacología, Virología, Endocrinología experimental, etc.- - y se participa en programas epidemiológicos de la Generalitat, como el Cronicat o el Monica—Catalonia. En el año 1978, se crea en el Sant Pau el primer servicio de Sugerencias y Reclamaciones del Estado, y en el año siguiente fue 
el primer hospital a publicar una Carta de derechos de los usuarios de la sanidad, que después abandonó en favor del texto común de la Generalitat, en el cual participa. Por otro lado, desde el año 1981, funciona un programa de calidad asistencial, con una metodología de autoevaluación del trabajo de los profesionales para el control de aspectos de riesgo.

\subsubsection{MISIÓN}

En 1401 con la constitución del hospital se definió como misión: servir a los pobres y peregrinos según la caridad cristiana. En 1997 como primer paso para hacer posible una gestión estratégica del Hospital de la Santa Creu i Sant Pau, se redefine su misión: La misión del Hospital de la Santa Creu i Santa Pau es ser un hospital abierto a la sociedad y a su entorno sanitario, centrado en el cliente, competitivo y dinámico. Los objetivos institucionales son:

1. El principal valor de la institución es la capacidad de dar un servicio de calidad a los ciudadanos.

2. El hospital se siente responsable de la salud de la población de referencia y, por tanto, asegurará todas las modalidades asistenciales con medios internos y/o externos, en colaboración con la red asistencial.

3. Como hospital de referencia, el hospital desarrolla selectivamente productos integrados y excelentes dirigidos a un ámbito territorial más amplio.

4. Como hospital general básico, el mercado natural es Barcelona ciudad y principalmente los distritos de entorno del hospital.

5. El núcleo de la actividad del hospital es la asistencia, conjuntamente con las líneas de investigación y docencia.

6. La organización será competitiva, abierta y dinámica, donde la información y orientación al cliente serán factores claves.

7. Los valores centrales del hospital son la excelencia en el servicio, en el trato y accesibilidad de la población.

8. El hospital se define como un centro de conocimiento.

\subsubsection{ESTRUCTURA Y ORGANIZACIÓN}

El Hospital de la Santa Creu i Sant Pau como institución de prestación de servicios sanitarios tiene el más alto nivel de la XHUP, con más de 600 años de 
historia es un Hospital emblemático para Barcelona y Catalunya y su calidad en todos los ámbitos le ha proporcionado prestigio en el ámbito internacional.

- Área de infuencia: Gràcia, Horta-Guinardó, Sant Martí, Dreta de l'Eixample y Sant Andreu.

- Tipología: Hospital de alta tecnología.

- Número de camas: 723.

- Servicios: Todas las especialidades.

- Áreas de investigación:

Área 1: Investigación cardiovascular.

Área 2: Investigación oncología y oncohematologia.

Área 3: Investigación neurociencias.

Área 4: Investigación clínica-diagnostica.

Área 5: Investigación fármaco y terapéutica.

FIGURA 6.38. Organigrama del Hospital de la Santa Creu i Sant Pau.

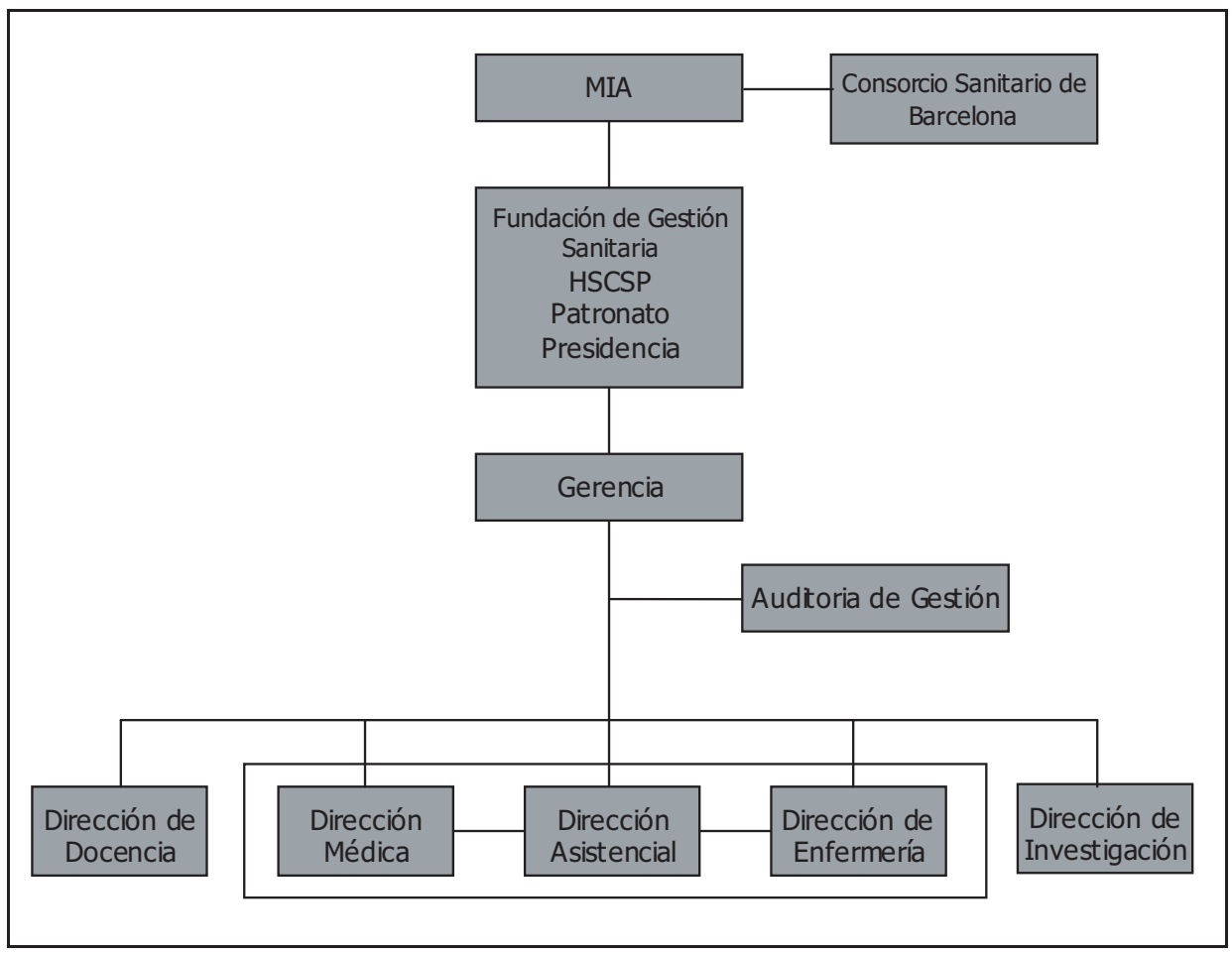

(C) Memòria 1996-1997 Hospital de la Santa Creu i Sant Pau [1998, p. 17]. 


\subsubsection{FUNDACIÓ PUIGVERT, IUNA}

\subsubsection{ANTECEDENTES HISTÓRICOS}

La Fundació Puigvert, fiel al espíritu de su impulsor, se distinguió desde su constitución en el año 1961, y más sólidamente todavía desde la inauguración de las instalaciones actuales en julio de 1966, por su carácter pionero. Esta característica le ha servido para contar con un alto prestigio internacional, tanto por la calidad asistencial como por las líneas de docencia e investigación. El Institut d'Urologia, Nefrologia i Andrologia —IUNA — fue el primer hospital privado que establece un concierto con la Seguridad Social, en agosto de 1967. Desde entonces, el centro ha compaginado la asistencia particular con la participación en el sistema sanitario público.

En el marco de la XHUP, el IUNA se inscribe como hospital de alta tecnología, integrado en la oferta médica y universitaria del Hospital de la Santa Creu i Sant Pau. La singularidad del hospital se caracteriza por su especialización en los campos de la Urología, la Nefrología y la Andrología, disciplinas que ha desarrollado notablemente o que, como en el caso de las dos últimas, ha contribuido a hacer nacer y prestigiar internacionalmente. Su equipo médico, que combina la superespecialización con el trabajo en equipos multidisciplinarios, ha sido y es un factor determinante de su personalidad.

El núcleo originario de esta institución se encuentra en el año 1943, cuando el profesor Antoni Puigvert crea, dentro de su clínica privada, el Institut d'Urologia. Ya de entrada cuenta con una doble función asistencial y docente, con 12 de las 38 camas y 3 de los 6 consultorios dedicados a enfermos de posibilidades económicas limitadas, y con una buena dotación tecnológica —un moderno aparato de radiología, equipos endoscopicos y una zona de anatomía patológica-.

Las ideas fundamentales del centro actual ya estaban en el primer proyecto. Para la enseñanza, se habilitó una amplia sala de operaciones como anfiteatro para el seguimiento de las intervenciones y Puigvert aportó una importante biblioteca y filmoteca quirúrgicas, y un archivo de historias clínicas desde 1928. Esto fue un punto de referencia único en la Urología española.

El primero Institut contaba con un equipo de dedicación plena, donde Puigvert implantó el principio de la formación continuada. Desde 1944, sistemáticamente, se hacían sesiones con todos los médicos para analizar los casos clínicos de interés y todos los que necesitaban cirugía. Se establecía, así, un 
mayor control de calidad. En el año 1946 se inicia formalmente la actividad docente con el primer Curso monográfico de Urología, todavía vigente y del cual se han celebrado ya 47 ediciones. En esta época, el profesor Puigvert viajaba constantemente a Suramérica, donde impartía cursos, presentaba ponencias, operaba enfermos y recibía honores y distinciones.

En 1951, Antoni Puigvert consigue la plaza de jefe del servicio de Urología del Hospital de la Santa Creu i Sant Pau, donde ejercía desde 1933. Tres años después, la Molt Il.lustre Administració del Hospital autoriza el traslado del Institut d'Urologia al Pabellón de la Assumpció, que se amplía para acoger las secciones de urografía, laboratorios y endoscopia, la biblioteca y el archivo clínico. Se implantan las sesiones clínicas y se inician las primeras publicaciones propias. Aquel mismo año 1954 se diseña un plan de estudios de la especialidad y se crean dos plazas de residente-becario.

El Institut d'Urologia del Sant Pau fue el primero de España a ofrecer un programa de formación reglada de la enseñanza de especialización para postgraduados. Con los trabajos sobre urografía intravenosa y la aplicación de las primeras técnicas de diagnóstico por la imagen, Puigvert impulsó el surgimiento de la Nefrología, partiendo de la base que debía considerar como un conjunto los riñones y las vías excretoras. En el año 1955, el Institut abre el primer consultorio de la especialidad, y en 1960 se construye la «Aula Serés», se amplía el plan académico a tres años, y se convocan tres plazas para residentes-becarios.

El año 1961 es una fecha decisiva en tres sentidos. Por un lado, se crea la sección de Andrología. Por otro, se establece un convenio entre el profesor y el Sant Pau para volver a ampliar el Pabellón y para construir dos nuevos edificios. Y, en tercer lugar, se constituye la «Fundació Puigvert», como entidad particular de carácter benéfico y docente. Puigvert vende su clínica de la calle de Provença, acordando un pago avanzado con una entrega aplazada. En el año 1963 comienzan las obras de la actual sede del Institut-Fundació. La actividad hospitalaria, paralelamente, experimenta un aumento considerable. En el año 1964, con 62 camas y 6 consultorios, se amplía el equipamiento técnico. Se construye una sección de hemodiálisis con tres riñones artificiales de placas. El plan de especialización de Urología pasa a durar cuatro años y admite cuatro residentes por año. En 1965 se constituye el primer servicio de Nefrología en España, se define el plan de especialización y se convoca, en el año siguiente, la primera plaza de residente. En julio de 1966 se inaugura oficialmente los nuevos edificios. Seguidamente, se pone en marcha el nuevo 
bloque quirúrgico — uno de los primeros con circuito interno de televisión-y se abre la clínica y la consulta particular. El personal de la Clínica Puigvert se integra en la Fundació y los médicos trasladan su actividad privada, cediendo a la entidad un porcentual de los sus honorarios. En aquel diciembre, el centro ofrece 75 camas de uso público y 65 de uso privado, dispone de cuatro salas de radiología, dos de endoscopia, cuatro laboratorios - Hematología, Bioquímica, Microbiología y Anatomía patológica-, tres salas de operaciones y una central de esterilización. Trabajan 147 laborales, 24 médicos con contrato de servicios y 20 residentes_-becarios. Su presupuesto anual es de 47 millones de pesetas, lejos todavía de los más de 4.000 millones actuales.

En el año 1967, con una gran visión de futuro, se firma un convenio con el Instituto Nacional de Previsión - INP_. Aquel mismo verano, por otro lado, la universidad reconoce el trabajo docente de la Fundació y declara el Institut escuela de especialización para postgraduados — adscrita actualmente en la Universitat Autònoma de Barcelona-. El interés por la infertilidad masculina había dado lugar a un grupo de trabajo con una gran experiencia, se habían integrado disciplinas y se había establecido contacto con equipos de investigación básica. En enero de 1968, el Institut crea el primer servicio de Andrología del mundo. Como en el caso de la Nefrología, se diseñó un plan de especialización y se convocó la primera plaza de residente, en el año 1969. En el año siguiente se impulsa la creación del Comité Internacional de Andrología —CIDA - y en 1976 se celebró en Barcelona el primer congreso internacional de esta disciplina. En el año 1981, el CIDA se descompone en favor de la Sociedad Internacional de Andrología.

Entre 1968 y 1970 se transforman camas privadas en camas públicas, por causa del gran crecimiento de la demanda de este sector y también por la dificultad que comportaba en aquel momento la atracción de pacientes privados a un centro hospitalario.

En el año 1971 se funda la revista Anales de la Fundación Puigvert que, desde 1982, lleva el nombre de Actas de la Fundación Puigvert. Aquel mismo año se incorpora al cuerpo facultativo un psicólogo clínico, para paliar el sufrimiento emocional de los pacientes que siguen programas de hemodiálisis crónica y para la preparación de las cirugías de tipo mutilante. En la actualidad, en el centro funciona un servicio de Sicología clínica.

El nombre actual de Institut d'Urologia, Nefrologia i Andrologia - IUNA— fecha del 1972, año en que también se instituye la Medalla Francisco Díaz, padre de la Urología, un premio valorado internacionalmente. Es importante destacar 
el esfuerzo realizado en informatización —el primer ordenador central es de 1972- y en los sistemas de información asistencial —en el año 1978 se crea el servicio de documentación médica-.

Otra línea de transformación es el paso del cuerpo facultativo a un régimen de contratación laboral, mayoritariamente con dedicación exclusiva, y el establecimiento de un sistema retributivo ligado a la experiencia y la capacidad profesional de los médicos —el que, en la actualidad, se conoce como «carrera profesional»—.

Entre 1977 y 1979 se superan las dificultades financieras del momento con una ampliación de la dotación fundacional. En el año 1985, como consecuencia de la crisis general de la financiación de la sanidad pública en Catalunya, la Fundació elabora un plan estratégico para el período 1986-1992. El plan promueve una más clara diferenciación entre la actividad de los sectores público y privado. Se adquirieren locales próximos y se instalan servicios diversos, entre los cuales destaca un área de enfermos crónicos para la hemodiálisis con dos zonas de ocho riñones artificiales. El plan impulsa una fuerte inversión tecnológica entre 1987 y 1990. Un primer litotriptor por ondas de choque y un segundo equipo instalado en una unidad móvil —la primera de Europa-, una fuente láser, un angiógrafo, ecógrafos, autoanalizadores, un nuevo ordenador central, el cableado informático del edificio, también un nuevo bloque quirúrgico y una unidad de Reanimación con 12 camas monitorizadas.

En el año 1985, el profesor Puigvert renuncia al cargo de presidente de la Fundació para dar el relevo a los miembros de su equipo. En 17 de mayo de 1990 muere en su hospital, donde perdura una estructura organizativa y funcional propia, que fue fijada en el año 1988 en un documento nombrado Marco de referencia institucional, en que se definen la misión, la cultura y los principios de la institución.

\subsubsection{ESTRUCTURA Y ORGANIZACIÓN}

- Área de influencia: Catalunya.

- Tipología: Hospital de alta tecnología.

- Número de camas: 165.

- Servicios: Urología. Nefrología. Andrología y Uroradiología. 


\subsubsection{HOSPITAL CLÍNIC I PROVINCIAL DE BARCELONA}

\subsubsection{ANTECEDENTES HISTÓRICOS}

El proyecto de un nuevo hospital universitario en la Barcelona del último tercio del siglo XIX, que avanzaba con grandes dificultades, sumase al empujón que impulsaba la ciudad desde el entusiasmo de la Exposición Universal de 1888. Se aprovecha la presencia de la Reina Regenta y de su comitiva, y el 17 de mayo de aquel año, en nombre de la reina, el ministro de Fomento coloca la primera piedra. La facultad de Medicina y el nuevo hospital no abren sus puertas hasta los años 1906 y 1907, respectivamente. Después de unas primeras gestiones infructuosas, en 1879 el claustro de la facultad aprueba y presenta al rector de la Universitat de Barcelona un dictamen sobre la necesidad de un nuevo edificio, tanto para la mejoría de la enseñanza como para la mejoría asistencial de la población, que contaba únicamente con las 800 camas de un antiguo y saturado Hospital de la Santa Creu. También, los universitarios querrían superar las limitaciones que el patronato de la Santa Creu ponía para el ejercicio docente. Se escogen las dos manzanas del Eixample que existía entre las calles de Casanova, de Provença, de Villarroel y de Còrsega. Se estudian diferentes modelos arquitectónicos —radial, cruzado, rectangular, poligonal—y la facultad opta por una disposición lineal doble, con pabellones paralelos y enlazados por galerías interiores, de acuerdo con los dictados de la Academia Francesa y el referente del Hospital Civil de Montpeller.

En enero de 1880, la dirección general de Instrucción Pública encarga el estudio económico y el proyecto del edificio, del cual se encarga Bartrolí, un arquitecto de la Diputación Provincial de Barcelona. En el año siguiente, el proyecto recibe el visto bueno de la junta Consultiva de Caminos, Canales y Puertos y de la Real Academia de San Fernando. En 26 de enero de 1882, el ministro de Fomento aprueba el proyecto. Después de la colocación de la primera piedra [1888], las obras no se inician hasta el 25 de junio de 1895, por problemas económicos.

La Diputación y el Ayuntamiento habían aportado dineros para la compra de los terrenos. La dirección de obra se encomienda a Josep Domènech i Estapà, arquitecto y catedrático universitario, autor o coautor de otros edificios públicos como el Palacio de justicia, la prisión Modelo, la Academia de las Ciencias o la sede de Catalana de Gas. Se levantan doce pabellones con capacidad para 500 camas, dos de ellos se quedan insolados para enfermedades infecciosas y los diez 
restantes, cinco y cinco, se reparten entre hombres y mujeres para la Medicina general y Cirugía. Se hacen ocho anfiteatros operatorios semicirculares, con luz natural — vertical y lateral—, emplazados en los patios entre pabellones, al lado cada uno de una sala de hospitalización de enfermos quirúrgicos. De entre las instalaciones destacaban las de terapéutica física —una de las mejores del continente-, hidroterapia, electroterapia, rayos X y neumoterapia, entre otras novedades funcionales como los ascensores eléctricos.

El 26 de julio de 1906, un real decreto nombra una junta administrativa, formada por cuatro miembros designados por el gobierno, un delegado del rector de la Universidad, un diputado provincial y un regidor del Ayuntamiento. La junta, actuando siempre en nombre del Estado, ha de confeccionar los presupuestos, dirigir los servicios administrativos y gestionar las aportaciones estatales, provinciales, municipales y particulares. El Estado es el propietario del edificio y se ocupa de la subvención de 200 camas. La Diputación y el Ayuntamiento son titulares de los terrenos. La Diputación, también, toma el acuerdo de utilizar el hospital como establecimiento provincial — una falta que arrastraba desde la Ley de Beneficencia de 1849 - y asume los costes del equipamiento hospitalario. El 2 de octubre de 1906 se inauguran las clases en la nueva facultad. Desde el Carrer del Carme, los estudiantes se trasladan en un tipo de desfilado festivo, compuesto por una decena de coches cargados de muebles y material diverso.

Entre los impulsores de esta puesta en marcha está el doctor Joaquim Bonet i Amigó, catedrático de Obstetricia, que fue rector de la Universidad entre 1905 y 1913. Y también el doctor Valentí Carulla i Margenat, catedrático de Terapéutica física, que fue presidente de la junta administrativa durante diecisiete años — más adelante también fue rector- y que hizo un trabajo de organización de los servicios, de mejoría del centro y de obtención de recursos. Finalmente, en el 1 de enero de 1907 entra en funcionamiento el hospital. Entre las primeras actividades científicas del centro, se celebra el Congreso de Electrología y Radiología [1910]. Como depósito judicial y centro de asistencia a presos y detenidos, el Clínic se encuentra involucrado en los principales acontecimientos de agitación social de la época, como la «Setmana Tràgica» [1909], la bomba en el «Café Pompeia» [1920] o el multitudinario entierro de Francesc Comas, un compañero de Salvador Seguí — por el cual se había prohibido el entierro público-, en marzo de 1923. De la gestión interna del hospital y de la asistencia, se ocupaba la «Reverenda Comunitat de les Germanes de la Caritat de Santa Anna». 
Durante los años de la Primera Guerra Mundial [1914-1918] se manifiesta la dificultad para ajustar los gastos y los ingresos. Las asignaciones del Estado —340.000 pesetas_, de la Diputación — 200.000 pesetas_ y del Ayuntamiento —135.000 pesetas — se habían de complementar con más de 300.000 pesetas anuales, provenientes de recaudaciones extraordinarias. De las 700 camas del hospital, los costes de unas trescientas dependían de la caridad de particulares. También, la junta adelanta dineros que después había de recibir del Estado con mucha demora, para poder abordar obras y mejoras. Estos dineros, junto con disposiciones testamentarias, permiten la construcción de cuatro salas para incurables y dos salas más para afectados por tuberculosis, mantenidos por dos benefactores particulares. Se incorporan, también, nuevos servicios como la clínica de dermatología, un dispensario de ginecología y de enfermedades del pecho, nuevas instalaciones de electroterapia y radioterapia, un dispensario de enfermedades digestivas, un dispensario odontológico y un laboratorio clínico.

La actividad del Hospital Clínic a lo largo de la Guerra Civil [1936-1939] fue muy intensa, tanto los días posteriores a la sublevación militar como durante los combates del mayo de 1937 o las ofensivas aéreas sobre la ciudad. Después de la guerra, los catedráticos que se habían comprometido políticamente hubieron de exiliarse o de mantenerse en una semiclandestinidad profesional. Sus plazas fueron cubiertas por concurso estatal, con titulares que ocupaban la plaza temporalmente para después irse a Madrid. Esta realidad acentuó la separación del Clínic respecto al modelo que podía representar el Sant Pau, con jefes de servicio de tradición barcelonesa.

En el año 1952, con una gran dificultad acumulada, un decreto gubernamental cambia la estructura jurídica del centro y le convierte definitivamente en Hospital Clínic i Provincial. La financiación de la institución queda repartida entre la Diputación —un 42\% - el Ministerio de Educación Nacional —un 22\%-el Ministerio de la Gobernación —un 22\% - y el Ayuntamiento de Barcelona —un 14\%—, mientras que este último no tenía representación en el nuevo Patronato. Un patronato que preside el gobernador civil — la Dirección General de Sanidad dependía del Ministerio de Gobernación-. Y que tenía como vocales el presidente de la Diputación y el rector de la Universidad, y que dividía las funciones directivas entre una junta administrativa —el presidente de la cual era nombrado por la Diputación-, y una junta de clínicas, presidida por el decano de la facultad de Medicina, para los aspectos docentes y facultativos. Por otro lado, un decreto del 10 de octubre de aquel año asigna al hospital la asistencia de los enfermos pobres de la provincia. En el año 1957, para el cincuentenario del 
hospital, se inaugura el nuevo anfiteatro. En el año 1963 la sexta flota norteamericana entrega como donativo un pulmón artificial. Hasta finales de los años sesenta se mantiene una estructura de funcionamiento por cátedras, entendidas como clínicas independientes, cada una con sus especialidades duplicadas. La junta de clínicas — los catedráticos — dan entrada a médicos hospitalarios elegidos democráticamente, que introducen elementos de renovación. En el año 1972 se presenta un libro blanco del hospital, como plan de futuro de un centro que había de asumir una plantilla jerarquizada. Se crean siete subdivisiones - Medicina, Cirugía, Ginecología y Obstetricia, Pediatría, especialidades médicas, servicios comunes y laboratorios- y se mantienen las clínicas regidas por catedráticos, pero con las especialidades repartidas entre ellos y sin duplicidades. Estas especialidades pasan a servicios y, más adelante, cátedras de especialidad.

Paralelamente, desde 1969, el colectivo de médicos internos y residentes reclaman la obtención de contratos laborales. En el año 1975, a este movimiento, se agrega la demanda de estabilidad y de mejoras saláriales para el conjunto del personal. Mientras tanto el hospital firmaba un convenio con la Seguridad Social [1973].

En el año 1975 se produce también una nueva transformación en los órganos degobierno, como primer centro hospitalario español que se abre a la participación de los diferentes sectores. La junta administrativa pasa a tener quince vocales, divididos en tres tercios: Los representantes de los diferentes estamentos de la plantilla del hospital, los representantes de los organismos oficiales competentes en sanidad —el Ayuntamiento, la Delegación Provincial de Sanidad, el Ministerio de Trabajo- y los de los usuarios. Estos vocales son, respectivamente, ratificados, nombrados y designados por el presidente de la Diputación.

En 1978, una donación privada permite la instalación de un servicio de Neumología, que es inaugurado el 30 de septiembre de aquel año por Josep Tarradellas, presidente de la Generalitat. Una orden ministerial, del 18 de marzo de 1983, modifica el reglamento de régimen interno del centro, crea la figura del gerente y regula las funciones de los diferentes servicios administrativos. Por otro lado, aquel año la situación de los edificios del hospital presentaba problemas de control de acceso y de circulación, de seguridad, de dispersión de unidades y de insuficiencia o falta de servicios básicos. Para conseguir los mínimos espacios por camas, el centro tenía una falta de diecinueve mil metros cuadrados. Por ésto se adquiere un terreno en las calles Casanova y Rosselló, para acoger las consultas externas, la escuela de enfermería, los archivos y los aparcamientos. Con esta compra se planteó 
una reordenación importante, se unifica la entrada del público en la Calle de Villarroel, se construyen salidas de emergencias, se habilitan pasadizos de uso público, se renuevan áreas de hospitalización, se agrupan quirófanos y unidades de curas intensivas, se agrupan laboratorios y se acercan a los usuarios las salas de extracciones y se centralizan las admisiones, entre otras medidas. En 1984, también, empiezan las obras de la facultad de Medicina, lo que motivó la necesidad de trasladar las dependencias situadas en el área docente, las consultas de rehabilitación — trasladadas a un local primero cedido y después adquirido por el hospital en 1987-, las consultas de psiquiatría — trasladadas del centro — y la sala de autopsias, construida de nuevo. En la junta del patronato del 19 de junio de 1984, se acuerda la reestructuración de la junta de clínicas, la cual sé efectiva en el octubre de 1986. También se conoce el decreto del 10 de junio de 1984 de la Diputación, del cambio de la junta administrativa.

A partir de la segunda mitad de los años ochenta, el Clínic refuerza el proceso de transformación con la reestructuración de su plantilla médica [1985], con la implantación de un plan general [1988] y con la redacción de un proyecto de empresa [1989], que define los principios para el desarrollo armónico y de calidad de las tres competencias fundamentales del centro —asistencia, docencia e investigación-.

Este documento planteaba el reto de la institución: «Hacer del Hospital Clínic i Provincial de Barcelona un hospital de vanguardia, símbolo de la contribución catalana a la medicina europea» [La Xarxa Hospitalària d'Utilització Pública de Catalunya. Història d'una diversitat, 1994, p. 78].

A principios de los años noventa, los objetivos de cambio fueron tomando forma, se ha habilitado una entrada directa al Centro de Diagnóstico y Tratamiento por el Carrer de Còrsega y se han renovado cerca de treinta salas de hospitalización [1993]. Desde 1992 se trabaja en la coordinación del grupo Corporación Sanitaria, que engloba otros centros como el Hospital Casa de Maternidad y la unidad integrada del Hospital de Sant Joan de Déu.

\subsubsection{ESTRUCTURA Y ORGANIZACIÓN}

El Hospital se está estructurando según el modelo denominado de organización enfocada al paciente en forma de Institutos y Centros a fin de realizar una asistencia lo más personalizada posible, de avanzar hacia una gestión descentralizada y de implantar progresivamente el modelo de «gestión clínica» 
con responsabilidades crecientes de los profesionales sanitarios en la gestión de recursos. En el Proyecto Prisma, después de un estudio exhaustivo, se perfiló en el enfoque organizativo siguiente:

- Junto con la Unidad Integrada con el Hospital Sant Joan de Déu, se crean los Institutos Clínicos de:

- Aparato Respiratorio.

- Aparato Locomotor.

- Enfermedades Cardiovasculares.

- Enfermedades Digestivas.

- Enfermedades del Sistema Nervioso.

- Ginecología-Obstetricia y Neonatología.

- Hematología y Oncología.

- Nefrología y Urología.

- Oftalmología.

- Psiquiatría.

Los cuales atienden a grupos homogéneos de pacientes. La organización se completa con el Centro de Especialidades que agrupa los servicios no dependientes de un Instituto y aglutina patologías de elevada prevalencia en la comunidad; el Servicio de Urgencias; dos Centros, de Diagnóstico Biomédico y de Diagnóstico por la Imagen; las Áreas de Atención Primaria y Sociosanitaria — Gesclínic- y, por último, las Unidades de Coordinación Asistencial y de Recursos y servicios Comunes necesarias.

Con la nueva organización se busca implantar la gestión clínica, transfiriendo responsabilidad de funcionamiento y presupuesto a cada Instituto o Centro.

- Área de influencia: Barcelonès.

- Tipología: Hospital de alta tecnología.

- Número de camas: 855.

- Servicios: Todas las especialidades.

- Áreas de investigación:

Área 1: Genética. Reproducción. Metabolismo y Oncogénesis.

Área 2: Bases celulares y moleculares de la respuesta inmune y autoinmunidad. Enfermedades infecciosas y SIDA.

Área 3: Fisiopatología y tratamiento de las enfermedades hematológicas.

Área 4: Fisiopatología y tratamiento de las enfermedades hepáticas, pancreáticas y gastroenterológicas.

Área 5: Fisiopatología de la hipertensión, arteriosclerosis y enfermedades cardiovasculares. 
Área 6: Fisiopatología y tratamiento de las enfermedades respiratorias.

Área 7: Neurociencias.

Área 8: Epidemiología y Salud Pública.

Área 9: Bioanalítica médica.

Área 10: Farmacología y toxicología.

Área 11: Neuroquímica.

Área 12: Patología molecular y terapéutica.

\subsubsection{HOSPITAL DE SANT JOAN DE DÉU}

\subsubsection{ANTECEDENTES HISTÓRICOS}

Ubicado sobre el límite municipal de Barcelona y Esplugues de Llobregat, se inaugura el día 3 de febrero de 1973 el actual Hospital de Sant Joan de Déu, un centro de primer orden en la atención maternal y pediátrica. Recoge toda la tradición del primer hospital infantil fundado en España, en el año 1867, por Benet Menni, un milanés que fue enviado para refundar la orden de los Hospitalarios, una organización confesional que cuenta en la actualidad con más de doscientos centros repartidos entre cerca de una cincuentena de países —instituciones médico/quirúrgicas, psiquiátricas, geriátricas y de educación especial y de acogida de indigentes- El fundador de la orden, el portugués Juan Ciudad, conocido como Joan de Déu, había instaurado en 1539 el hospital de Granada, donde se origina la comunidad de sus seguidores. En el año 1597 dos hermanos llegan a Barcelona, con la intención primera de integrarse en el Hospital de la Santa Creu y, después, de abrir un asilo de convalecientes en la Rambla. En 1598, por problemas con los consejeros de la ciudad, abandonan el establecimiento y lo integran en la Casa de Misericòrdia. En España, sin embrago, la orden se había extendido y contaba con diversos centros.

A partir de 1835, pero, como consecuencia de las disposiciones gubernamentales de exclaustración, la congregación se fue extinguiendo por causa de la prohibición de tener nuevos novicios. En el año 1850 muere el último prior general de la orden en el Estado. Doce años más tarde, el general de la orden en Europa central, el hermano Alfieri, intenta la recuperación. En Girona y en Barcelona propone a los hermanos de la Caridad, de los dos hospitales generales, que se integren en su orden, pero las gestiones no 
prosperan. Con todo, establece contacto con miembros de la sociedad barcelonesa, vinculados al Hospital de la Santa Creu y en las acciones benéficas - Josep Blanquet, capellán de la Casa de Convalescència; Nonit Plandolit, adinerado comerciante, y una junta de señores, de entre los cuales destacaba Dorotea Chopitea, que tiene también un papel decisivo en la construcción del Hospital del Sagrat Cor y del Hospital de Sant Rafael_. En 1865, por medio de Francesc Bramon, un catalán integrado en la orden, se intenta el primer proceso fallido de implantación. Dos años más tarde, la orden envía a Barcelona el hermano Menni. Se instala en el Hospital de la Santa Creu donde ve la necesidad de un centro para niños enfermos, escrofulosos, raquíticos o disminuidos físicos. Plandolit pone los dineros para alquilar una antigua casa de comensales en la esquina de las calles Muntaner y Rosselló, entonces, situada en el término de Gràcia. El gobierno civil aprueba el reglamento en el mes de noviembre - la orden aparece camuflada como una «sociedad filantrópica extranjera»—. Así nace el Hospital de la Puríssima Concepció. De esta manera, pues, en 1867 se abre el primer centro monográfico infantil, con una dotación de una decena de camas. En el año siguiente se traza ya un plan de expansión pensando en un pabellón para 50 niños, con enfermería, farmacia, sala de recuperación, baños, gimnasio, escuela, camas para urgencias y espacio para doce hermanos y doce novicios. Sin duda, el establecimiento había creado mayor demanda. En 1869, en nombre de Plandolit, se compra la casa, y en 1870 se empieza una de las tradiciones de la institución, la enseñanza de la música como forma de preparación profesional de los niños ingresados. Después de un paréntesis, durante la república federal, en que los hermanos tienen que huir a Francia, entra en funcionamiento, a finales de 1875, la ampliación proyectada.

En el año 1877, un fuerte endeudamiento contraído en los negocios por Nonit Plandolit obliga a vender el edificio y tener que buscar uno nuevo. La casa ya acogía, entonces, alrededor de un centenar de niños. El solar escogido fue nombrado Prat d'en Rull, al lado de la carretera de Sarrià, dentro del término de las Corts. En 1881 se hace el traslado a la casa de la finca, que se convierte en el Asilo de la Puríssima Concepció de les Corts.

En el 26 de febrero del año siguiente se pone la primera piedra de lo que había de ser un edificio suficiente para la creciente demanda. Las obras fundamentales se terminan en el año 1908.

Se albergaban unos doscientos cincuenta niños entre cinco y dieciséis años, distribuidos en cuatro grupos — los inválidos, los escrofulosos, los tiñosos, que 
estaban aislados por el carácter contagioso de la enfermedad, y los ciegos, que se habían incorporado en el centro en el año 1887. Se les daba escolaridad y, a los mayores, se les enseñaba un oficio en las oficinas de sastrería o zapatería. A los ciegos se les encaminaba prioritariamente a la música, y en buena parte se les ocupaba como profesores de música en hospitales de la orden, no se admitían niños con trastornos mentales nerviosos, ni los afectados por la tuberculosis pulmonar o enfermedades agudas infecciosas-.

Hasta los años treinta, el establecimiento se puede describir como hacía su médico director, Joaquim de Riba de Sanz — «desde el punto de vista médico, la Casa tiene la organización de hospital y sanatorio. Existen enfermerías de medicina y de cirugía, salas de aislamiento para enfermedades infecciosas y para enfermos graves, sala de curaciones, farmacia, laboratorios de análisis clínicos, sala de rayos $\mathrm{X}$, sala para especialidades [ojos, nariz, garganta y oreja, dentista] y departamento de operaciones»- [La Xarxa Hospitalària d’Utilització Pública de Catalunya. Història d'una diversitat, 1994, p. 126].

La estancia de los niños era gratuita y los únicos ingresos de la institución provenían de la caridad privada que obtenían los hermanos que andaban «de casa en casa y de puerta en puerta pidiendo limosna, no sólo por Barcelona, sino que también por toda Catalunya».

En el año 1923 la institución adquiere la finca Bona Vista en Calafell, con una masía y terrenos de cultivo, y en el año siguiente se estrena como colonia de verano. Al lado de la playa se construye un pabellón de fusta desmontable, apto para doce camas.

En 1926 se compra una finca en la orilla del mar y se empiezan las obras para un pabellón, con capacidad para cien camas, que se inaugura en el mes de mayo de 1929 por el Rey Alfonso XIII y la Reina Victoria Eugenia. A parte de acoger los niños acogidos en el verano, de acuerdo con los principios de la helioterapia, el sanatorio se había de destinar permanentemente a los afectados de tuberculosis ósea —en Barcelona, solamente había el Sanatorio Marítim de Sant Josep, para niñas y mujeres- La obra fue posible gracias a la caridad recogida repartiendo el folleto «Limosna de Amor», escrito por Amàlia Porqué, en el legado testamentario que hace en favor del hospital la señora Josepa Sanpere, y finalmente, los dineros obtenidos por la expropiación de una parte del terreno de las Corts para hacer pasar la calle Diagonal.

Efectivamente, en el año 1927, la Reina inaugura ya el acceso desde la nueva avenida, y en 1930, entraba en funcionamiento un nuevo cuerpo en aquella 
esquina. Aquel mismo año, los hermanos reciben la herencia de Francesc March i Muntada, con la misión de hacer un sanatorio para niños, preferentemente en Manresa. Con capacidad para 30 niños, se abre en el mes de junio de 1932. Desde 1993, el centro continua en activo formando parte del consorcio del Hospital General de Manresa.

A parte de unos incidentes sin consecuencias durante la «Setmana Tràgica» [1909], el Asilo Hospital de Sant Joan de Déu no vive ningún otro conflicto hasta el julio de 1936, en que los hermanos tienen que abandonar el centro. Los supervivientes se reintegran en el enero de 1939 y emprenden la reconstrucción de las partes deterioradas durante la guerra, especialmente las dedicadas al culto. Empieza entonces un período de cambio, y el centro se fue orientando hacia la atención pediátrica general, complementando el internamiento con el refuerzo de los consultorios externos para las familias con pocos recursos. Funcionalmente, este proceso comporta la reordenación del edificio, con la reducción proporcional del espacio para la hospitalización en favor de los espacio de diagnóstico y tratamiento.

El año 1954 es la fecha clave de este proceso. La campaña benéfica de Radio Nacional de España en Barcelona, impulsada por Dalmau i Viñas, dos clásicos de la radio de la época, posibilita la transformación en un verdadero hospital infantil —el que era un asilo/hospital con una atención prioritaria para las enfermedades crónicas-. Hasta entonces se había hecho una buena especialización en enfermedades osteoarticulares, tanto las infecciosas como las congénitas y las traumáticas. Con los dineros de la campaña radiofónica se inician las obras - un auditorio para las sesiones científicas, nuevos dispensarios, espacios quirúrgicos, servicios de recuperación funcional, etc. - y se adquieren nuevos instrumentos. Se abre, como tal, un servicio de Pediatría, uno de Urología infantil, uno de Cardiología y uno de Oftalmología, que adquirió un gran prestigio en el tratamiento del estrabismo. Con lo referente a los servicios existentes, como los de Neurología y de Odontología, experimentan mejorías tecnológicas, o como el de Otorrinolaringología, que instaló innovadores microscopios para la audiocirugía.

En el año 1963 se constituyó un servicio de Psiquiatría y Sicología infantil, con un equipo de treinta personas especializadas en psicometría, psicoterapia, trabajo social, logopedia y tratamiento de patologías del lenguaje — dislexia, dislalia-.

En el año 1964, la Sociedad Catalana de Pediatría promueve una encuesta entre todos los médicos pediatras comarcales. Una de las conclusiones de esta 
encuesta fue el descubrimiento de «zonas sanitarias cero». La primera zona incluía Badalona, Sant Adrià, Santa Coloma de Gramenet, Montcada i Reixac y el distrito $\mathrm{X}$ de Barcelona. Para una población de 470.222 personas, solamente había las 39 camas pediátricas del Hospital del Mar.

La segunda zona comprendía una población de 521.140 personas y afectaba a dos distritos de Barcelona - Poble Sec y Sants_y las poblaciones de l'Hospitalet, el Prat y Esplugues de Llobregat. Incluyendo todos las camas de Cirugía y Maternidad de las clínicas privadas — 106 camas- no había una sola cama para hospitalizar un niño. Una conclusión evidente fue la necesidad de promover hospitales infantiles y secciones pediátricas en los hospitales generales.

Sant Joan de Déu había de crecer. Se debatía entre continuar en las Corts, en una zona que se urbanizaba rápidamente y que encajetaría el centro, o trasladarse a la actual ubicación de Finestrelles, opción que favorecía los resultados del estudio. También, se situaría en un núcleo de comunicaciones de gran futuro, con la autopista y el segundo cinturón enfrente. El hecho de ser un edificio elevado y aislado también era importante. Se planeó, entonces, un centro todo nuevo apto para trescientas camas, y ampliable hasta cuatrocientas. El 30 de junio de 1969 empiezan las obras. Después de la venta del solar de las Corts, para la realización del nuevo proyecto faltaban unos cien millones de la época. Se resuelve hacer una intensa acción informativa y de relaciones públicas para la obtención de los recursos —anuncios en los diarios, paneles, carteles, publicidad radiofónica, conferencias, torneos deportivos, subastas de obras de arte, etc.- , junto con una nueva acción de Radio Nacional de España. A finales de 1972 se abren los consultorios y los laboratorios, y se trasladan los primeros niños para la hospitalización. El día 3 de febrero de 1973 se inaugura oficialmente el nuevo Sant Joan de Déu. Y en 1974 se abre la clínica maternal, donde el centro incorporaba los servicios obstétricos y ginecológicos, y se ampliaba el universo asistencial desde el nacimiento hasta los dieciocho años.

Con el nuevo centro nace también una importante institución - la Associació de Voluntaris de Sant Joan de Déu—, que desde 1973 funciona con unos estatutos que la reconocen como una entidad civil y aconfesional. Actualmente cuenta con 200 asociados. La acción del voluntariado, integrada en los equipos asistenciales, aporta servicios complementarios, destinados a una mejoría cualitativa de la asistencia al niño y a su familia, en los aspectos humanos. El voluntariado ofrece compañía, distracción, servicios de soporte 
a la familia, actividades lúdico-recreativas por medio de la biblioteca ambulante, ludoteca, concursos, etc., favoreciendo una estancia activa y con elementos positivos.

En el año 1976 la orden crea la obra benéfica de ayuda social destinada a la atención de aquellos pacientes que no disponen de seguro público ni de medios particulares. En 1979, en el barrio viejo de Barcelona, la orden abre el Albergue de Sant Joan de Déu, en coordinación con los servicios sociales de la ciudad, para acoger transeúntes. En la actualidad dispone de una unidad de Curas paliativas para enfermos del SIDA. Con relación a los aspectos docentes, el nuevo conjunto ofrece una amplia instalación educativa. Se incorpora la escuela de ayudantes técnicos sanitarios, que funcionaba ya en las Corts desde 1962 y que se transforma en la Escuela Universitaria de Enfermería [1979], masculina y femenina, adscrita a la Universitat de Barcelona. En 1973 se pone en marcha la Escuela Profesional de Pediatría y Puericultura, y en el año siguiente, la Escuela Profesional de Otorrinolaringología. Se imparten también clases de quinto curso de Medicina, en las especialidades Pediatría, Ginecología y Obstetricia. También se participa en programas de postgraduación para médicos internos y residentes - MIR—, en las especialidades de Pediatría, Obstetricia, Cirugía pediátrica, Bioquímica y Microbiología —en 1993 se firma un acuerdo con la Universitat de Barcelona, por el cual se reconoce el centro oficialmente como hospital universitario-.

El hospital emprende un proceso de jerarquización de servicios y de equiparación salarial con las instituciones cerradas de la Seguridad Social desde el 1978. Por otro lado, la incorporación de nuevos servicios, técnicas y especialidades — hasta las cuarenta actuales — ha estado notable — se reestructura el servicio de Urgencias [1977-1984], se crea el servicio de Planificación familiar [1980], se introduce en el centro el primer aparato de hemodiálisis [1983], se define un plan de control de calidad [1984], se abren los hospitales de día de Psiquiatría y Oncología [1990], y se crea la primera unidad de Curas paliativas pediátricas [1991]—.

En el año 1981 se crea el comité de ética del hospital, compuesto por catorce profesionales de los diferentes sectores del centro, que ha tenido un carácter pionero y que ha emitido importantes protocolos sobre temas como la adopción, la transfusión sanguínea en enfermos que la rechazan por creencias religiosas, los criterios de muerte cerebral de un niño para efectos de una posible donación de órganos, el consejo diagnóstico y el diagnóstico prenatal, entre otros. 


\subsubsection{ESTRUCTURA Y ORGANIZACIÓN}

En marzo de 1992 con la firma de un acuerdo entre la Generalitat, el Hospital Clínic y el Hospital Sant Joan de Déu se constituyó la Unidad Integrada de la Corporació Sanitària Clínic con objeto de actuar de forma coordinada y conjunta en las áreas de pediatría y cirugía pediátrica, de modo que pasaron a integrarse los servicios de Pediatría y Cirugía Pediátrica de ambos centros, realizándose este conjunto de actividades en las dependencias del Hospital Sant Joan de Déu.

FIGURA 6.39. Organigrama del Hospital Sant Joan de Déu.

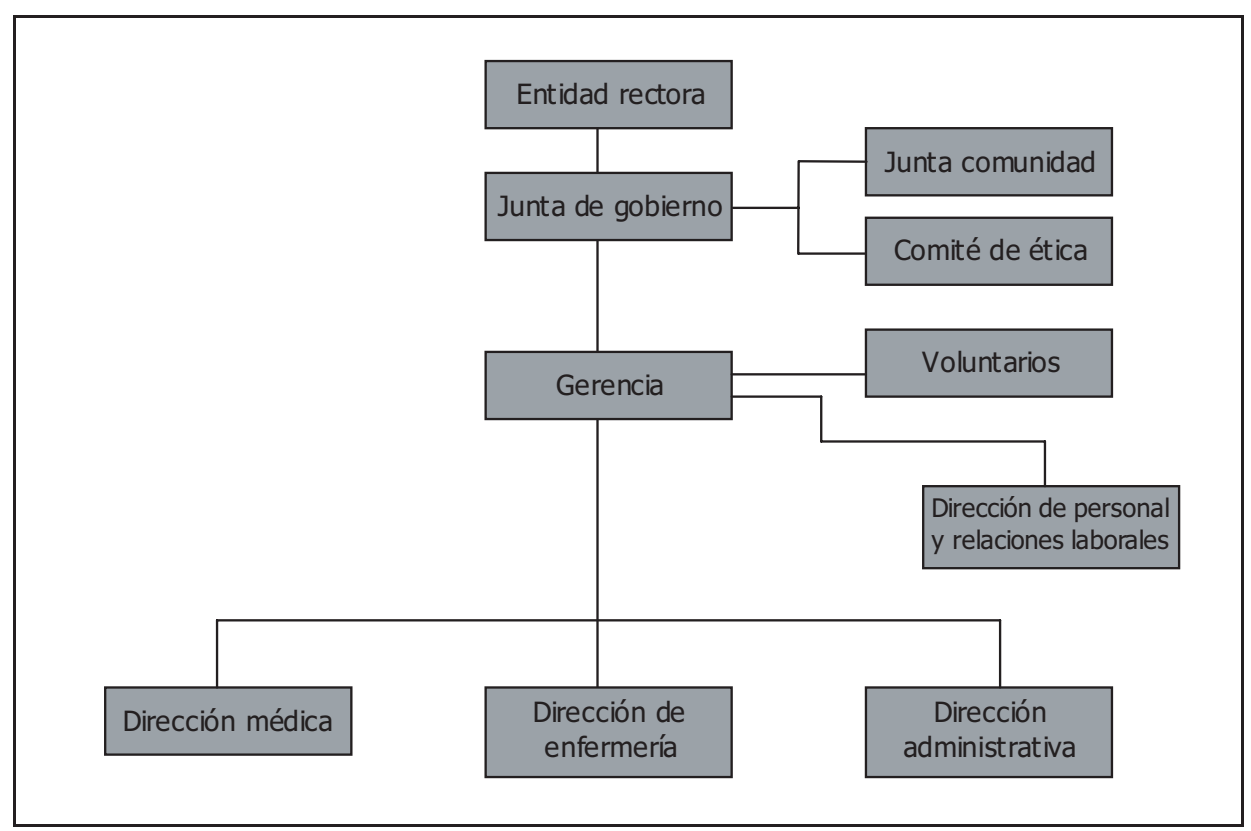

(C) Memòria Hospital Sant Joan de Déu 1997 [1998, p. 11].

En 1996 se traspasó al Hospital Sant Joan de Déu la cirugía cardíaca extracorpórea infantil, que desde la firma del acuerdo se venía realizando en el Hospital Clínic.

- Área de influencia: Baix Llobregat, Barcelonès.

- Tipología: Hospital de alta tecnología.

- Número de camas: 363.

- Servicios: Pediatría y sus especialidades médicas y quirúrgicas. Ginecología y Obstetricia. Neonatología. Unidad de Curas intensivas pediátrica. Psiquiatría. Psicología infantil. 


\subsubsection{HOSPITALES DE LA VALL D'HEBRON}

El conjunto sanitario de la Vall d'Hebron es el área de servicios asistenciales de mayores dimensiones de Catalunya. Cuenta con más de mil cuatrocientas camas, atiende más de doscientas veinte mil urgencias anuales, ingresan — también anualmente_ más de cincuenta mil personas y trabajan más de seis mil quinientos profesionales.

En la actualidad, forman parte tres centros hospitalarios: El Hospital General, el Hospital de Traumatologia i Rehabilitació y el Hospital Materno-infantil. A estos tres se tiene que sumar, también, el Hospital Quirúrgic Adrià que está ubicado en un otro punto de la ciudad, pero, trabajan en coordinación.

Por otro lado, en el mismo entorno conviven otros servicios como el Banc de Sang y el Institut Català de la Salut _ ICS—, ampliamente modernizado en 1992, una Unidad de Anatomía Patológica, Microbiología y Parasitología, de uso común, un centro de atención primaria del ICS, y otros servicios especializados como los de Farmacología clínica, Medicina preventiva o Medicina legal.

Desde el punto de vista docente, se ofrece formación de pregraduación y de postgraduación para los estudios de la Facultat de Medicina de la Universitat Autònoma de Barcelona —UAB — y dispone, desde 1966, de una Escuela Universitaria de Enfermería propia.

\subsubsection{ESTRUCTURA Y ORGANIZACIÓN}

Los Hospitales Vall d'Hebron como instituciones de prestación de servicios sanitarios pertenecientes al ICS tienen el más alto nivel de la XHUP. En este entorno con un alto grado de complejidad requiere unos esfuerzos de innovación y transformación por los cuales está pasando el Vall d'Hebron.

El nuevo modelo organizativo que se está desarrollando tiene un fuerte carácter descentralizador que debe permitir:

- Esclarecer y ordenar las diferentes líneas de prestación de servicios/ gestión dentro de los Hospitales Vall d'Hebron.

- Establecer un marco de asociación estable con otros proveedores sanitarios.

- Consolidar un entorno de trabajo más ágil y flexible para realizar proyectos de mejora de resultados. 
FIGURA 6.40. Organigrama general de los Hospitales de la Vall d'Hebron.

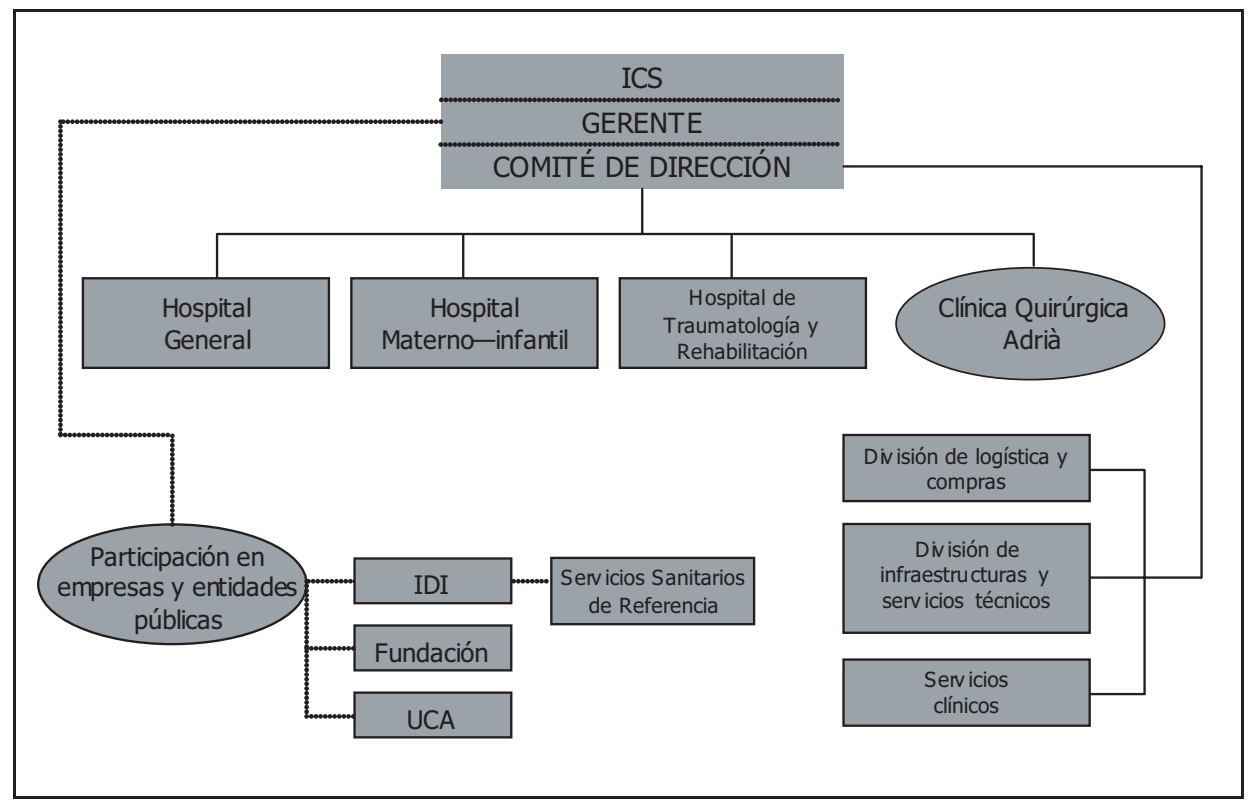

(C) Memòria 1997 Hospitals Vall d'Hebron [1998, p. 8].

\subsubsection{HOSPITAL GENERAL VALL D'HEBRON}

\section{Antecedentes históricos}

Históricamente, el conjunto se inicia con el Hospital General Vall d'Hebron, un centro de máximo nivel asistencial, dotado de todas las especialidades médicas y quirúrgicas — hasta que se traspasa a los hospitales monográficos- y de los más avanzados medios tecnológicos.

Fue inaugurado el día 5 de octubre de 1955, por el general Francisco Franco, y por el ministro de Trabajo, impulsor de las obras del Seguro de Enfermedad, José Antonio Girón de Velasco.

La construcción se prolongó entre el agosto del 1948 y el noviembre del 1954. Según el folleto que se distribuyó para la inauguración, para construir este edificio de 13 plantas de altura se hicieron servir cuatro millones de ladrillos agujereados, dos millones de ladrillos macizos y dos millones trescientas mil unidades de plaqueta. También daba otras cifras: 1.975 ventanas de carpintería metálica, 2.760 puertas de diversas maderas y 1.388 radiadores.

Entre las innovaciones para el bienestar se puede destacar la red general de megafonía para transmitir música o programes de radio en las habitaciones. En 
general, las plantas se distribuyen en tres habitaciones de cuatro camas, tres habitaciones de seis camas y cinco habitaciones dobles. Toda la ingeniería del centro, desde el monta literas hasta la central de mantenimiento de las instalaciones, se hizo también de acuerdo con las últimas novedades.

Arquitectónicamente, la residencia sanitaria — que recibía este nombre en oposición a la connotación negativa que tenia el término «hospital», ligado a beneficencia- define un modelo, junto con los otros centros del Estado.

Es el que se nombraba «la época del ladrillo» para la utilización de este material en las fachadas de obra vista. Son construcciones de gran solidez. El centro empieza a funcionar, de hecho, durante la primavera del 1955.

De acuerdo con la cobertura que ofrecía el Seguro Obligatorio de Enfermedad — SOE_, su planteamiento asistencial era eminentemente quirúrgico y funcionaba como centro abierto, es decir que, a parte de los cinco médicos de guardia de veinticuatro horas que atendían las urgencias, el resto de los facultativos eran médicos de ambulatorio - un término forjado también en esta época- que operaban en la residencia e iban a pasar visita a sus enfermos.

Hasta entonces el SOE atendía sus afiliados en clínicas concertadas. Sus residencias se definieron por imitación del modelo de la medicina privada, pero con unos núcleos de decisión muy centralizados y con un funcionamiento extremamente burocratizado.

A partir del año 1966, coincidiendo con la entrada en vigor de la nueva Ley de Seguridad Social, la entonces residencia cambia a Ciudad Sanitaria. Se construyen tres nuevos centros monográficos, dedicados a Traumatología, Pediatría y a Obstetricia y Ginecología, como prolongación física del Hospital General. Es el origen de los actuales Hospital de Traumatologia i Rehabilitació, y Hospital Materno-infantil. Los efectos de la inmigración y de la altísima natalidad favorecieron estos nuevos centros.

Durante los años sesenta se incorporan ya los primeros equipos quirúrgicos para atender urgencias de cirugía y los tres primeros médicos internistas.

En el año 1968 se emprende la jerarquización del departamento de Medicina interna, a cargo del doctor Agustí Pedro i Pons, que terminaba de jubilarse de su cátedra universitaria. Se desarrollan los servicios de Medicina interna y también las especialidades médicas.

Cuando fallece el profesor Pedro i Pons, en 1971, el doctor Joaquim Tornos asumió la jefatura del departamento. En el mismo año, el Hospital General se incorpora en la Universitat Autònoma de Barcelona - UAB - como centro universitario, y se empieza a perfilar una estructura organizativa que da como 
resultado el actual servicio de Medicina interna y especialidades médicas.

Por otro lado, en 1970, se jerarquizan los servicios quirúrgicos. El doctor Àngel Díez-Gascón es entonces el jefe de departamento. Se incluyen los servicios de Cirugía cardíaca, Oftalmología, Urología, Otorrinolaringología, Angiología y Cirugía vascular, Cirugía maxilofacial y Anestesióloga.

En el año 1972, se incorporan al departamento de Cirugía los jefes de Cirugía de urgencia, como jefes del servicio de Cirugía general. —La especialidad de Neurocirugía funcionaba como escuela profesional y no dependía del departamento-.

- Área de influencia: Barcelonès.

- Tipología: Hospital de alta tecnología.

- Número de camas: 721.

- Servicios: Todas las especialidades y Unidad de tratamiento de grandes quemados.

\subsubsection{HOSPITAL DE TRAUMATOLOGIA I REHABILITACIÓ VALL D'HEBRON}

\section{Antecedentes históricos}

El Hospital de Rehabilitació i Traumatologia — después se cambió la orden de las especialidades en el nombre- se inaugura en el 13 de junio de 1967. El incremento de los accidentes de tránsito y de trabajo fue el origen de este centro especializado que, desde el primer momento, contó con todos los servicios quirúrgicos y de rehabilitación necesarios. Los profesionales habían estado formados en Europa o en los Estados Unidos de América, o provenían de centros barceloneses de larga tradición, como el Sagrat Cor o el Clínic.

También desde su inicio contó con una plantilla jerarquizada. El centro adquirió un gran prestigio en la atención de lesiones medulares —en aquellos momentos, solamente el Institut Guttmann, inaugurado en 1965, se había especializado- y de parálisis cerebrales. Entre las patologías atendidas, desde el inicio, se debe destacar el tratamiento de las secuelas de la poliomielitis y también el tratamiento quirúrgico de la escoliosis, en que el hospital se destacó.

Después, la Cirugía ortopédica del centro evoluciona hacia la implantación de prótesis de cadera y de rodilla. Este camino hace un salto cualitativo con la introducción de las artroscopias. También, desde la apertura del hospital, se introduce la técnica de la osteosíntesis para el tratamiento de fracturas, una técnica desarrollada en Suiza. 
Con relación a los servicios centrales, el centro dispone de unidades de Curas intensivas, de un completo gimnasio de fisioterapia, de terapia ocupacional, de una novedosa unidad de postoperatorio y de un equipo de anestesistas, que con el tiempo han creado la actual Clínica del Dolor, que proporciona soporte a toda la Ciutat Sanitària, tanto para los enfermos ambulatorios como para los ingresados. Algunos servicios ocuparon temporalmente las instalaciones del Hospital de Traumatologia i Rehabilitació, como la Nefrología, la Neurología o el departamento de Cirugía plástica, que todavía mantiene una sección, y Quemados — que ha estado hasta 1975, año en que se traslada al Hospital General, después de su ampliación-.

- Área de influencia: Barcelonès.

- Tipología: Hospital de alta tecnología.

- Número de camas: 281.

- Servicios: Neurotraumatología. Traumatología y Ortopedia. Cirugía ortopédica. Cirugía plástica. Urología. Rehabilitación y Unidad de Curas intensivas.

\subsubsection{HOSPITAL MATERNO—INFANTIL VALL D'HEBRON}

\section{Antecedentes históricos}

En dos bloques diferenciados, el Hospital Maternal y la Clínica Infantil iniciaron sus actividades en el mes de mayo de 1966, pero fueron inaugurados el 13 de junio de 1967, en un contexto social de crecimiento migratorio y demográfico espectacular. Al principio de los años setenta se atendía una media de ochenta partos diarios. Los dos centros se unieron en 1988 para dar lugar al actual hospital universitario Materno-infantil. Este proceso de fusión motiva la necesidad de unas importantes obras — para remodelar y ampliar-, que han redefinido prácticamente la totalidad de sus espacios y que han ampliado el conjunto con tres plantas subterráneas, que acogerían, entre otros, la ampliación de las Urgencias, un nuevo bloque quirúrgico, Hospital de día, unidad de Curas intensivas, esterilización, cocina, vestuarios, archivo de historias clínicas y diagnóstico por la imagen. Con las obras se amplían para tres los espacios destinados a Consultas externas, que se quedan más amplios y confortables. Se potencia, en la nueva superficie, una gran importancia a las zonas de hospitales de día, para que los pacientes puedan recibir el tratamiento sin haber de quedarse por la noche en el hospital. También se dotan con unos espacios amplios el vestíbulo de entrada — donde se tiene quioscos de flores, prensa y 
regalos-, y los puntos de información, de atención al usuario y de trabajo social. También se remodelan las habitaciones, que se hacen para dos personas y con baño.

Con lo referente a las patologías, el centro evolucionó desde las infecciones clásicas — como la poliomielitis, el sarampión o la deshidratación- a nuevas enfermedades — la drogadicción, el SIDA — y a nuevos tratamientos, como los trasplantes. Entre las líneas en que se avanzó notablemente figuran el tratamiento del cáncer, los trasplantes, la atención global al fenómeno reproductor como la asistencia en la concepción, el diagnóstico prenatal y el embarazo de alto riesgo. Las unidades de Curas intensivas del hospital, tanto la neonatológica como la pediátrica, son de las mejores dotadas de Catalunya. Históricamente, el hospital marcó hitos en el Estado español como los de la primera hemodiálisis pediátrica [1970], el primer trasplante de riñón pediátrico [1981] y el primer trasplante de hígado [1985].

El centro cuenta, por otro lado, con un programa de calidad asistencial, que desarrolla actividades — tanto para los usuarios como para los profesionales- y trabajos en comisiones especializadas sobre aspectos como los tumores, las infecciones o los aspectos éticos de los ensayos clínicos. Otro de los programas del hospital es el de acogida, que gestiona visitas no asistenciales y coordina los voluntarios —entre otros, Cruz Roja, asociaciones de padres de niños oncológicos y diabéticos-.

Volviendo al conjunto de la Ciutat Sanitària, simultáneamente a la entrada en funcionamiento de los centros monográficos, se empezaba a forjar la siguiente generación de facultativos. La primera promoción de médicos internos y residentes — MIR — de la Vall d'Hebron es del 1968, donde se consolida como segundo centro de todo el Estado después de la clínica Puerta del Hierro. El programa MIR, dotado por la Seguridad Social, significó la regulación de la obtención de la especialización, en la línea de los países occidentales. Paralelamente, desde 1966, se había puesto en marcha la Escuela de Enfermería. Hasta entonces la residencia contaba con una comunidad religiosa y un grupo de enfermeras internas, responsables de planta, que vivían en el centro.

La Enfermería de la Vall d'Hebron implantó el horario de doce horas en turnos de tres días alternados en la semana y con los domingos salteados. El colectivo impulsó, también, una definición propia de enfermería que, entre otras cosas, decía: «Se debe conceptuar la Enfermería como una profesión de soporte para las personas, con tal de conseguir que se puedan valer por sí 
mismas, conseguir la autosuficiencia, independiente de las posibles deficiencias físicas o psicológicas. La enfermera ha de enseñar y ayudar a la persona a tener cuidado de élla misma, a ser independiente. Tener cuidado de la persona se ha de basar en considerarla como un ser biopsicosocial con la capacidad de marcarse objetivos, tomar decisiones, y con el derecho y el deber de escoger, de acuerdo con sus ideas y su propia escala de valores» [La Xarxa Hospitalària d’Utilització Pública de Catalunya. Història d’una diversitat, 1994, p. 110]. Este personal sanitario joven participó en las primeras plazas de los MIR [1971] y de enfermería [1972] en el Estado. Se producen despidos y se expedientan trabajadores que, posteriormente, serían readmitidos. Algunas de las mejorías obtenidas por la Enfermería, por ejemplo, fueron el reconocimiento del total del salario durante las bajas por enfermedad o la apertura de la guardería para el personal femenino, que actualmente continua en funcionamiento y para todo el personal.

En el año 1975 entra en servicio una nueva parcela del edificio del Hospital General, que se prolonga por uno de los extremos. En la ampliación se ubicó el servicio de Anatomía patológica, antes de disponer de un pabellón propio [1978]. Finalmente se destinaron tres plantas para las Consultas externas, dos para la unidad de Curas intensivas — UCI—, dos más para Nefrología, trasplantes renales y diálisis, una para Cirugía plástica, y una para la unidad de Quemados, un servicio de referencia para toda Catalunya.

En el año 1991 se pone en marcha la nueva ampliación de Urgencias, con una superficie de cuatro mil metros cuadrados. Esta obra fue el inicio de un ambicioso proceso de reformas, concretado en las unidades de hospitalización, donde se transformaron las habitaciones en dobles dotadas con baño. Con la transición democrática, se produce de forma tácita un cambio de nombre. Todos hablaban ya de la Vall d'Hebron. A partir de las transferencias sanitarias, y de la creación del Institut Català de la Salut [1983], los hospitales consolidan y amplían sus líneas de calidad.

Desde el punto de vista médico y quirúrgico, las aportaciones de sus profesionales son numerosas, ya con el inicio de la práctica de la Cirugía arterial directa, en el año 1970, para la corrección de la patología obliterante y aneurística. El Hospital General implanta programas de trasplante de riñón, de hígado — también se hace el primer multiorgánico de hígado y riñón, de España, en mayo de 1994-, de córnea, de médula ósea y de pulmón — se hizo el primer trasplante unipulmonar del Estado, en agosto de 1990—. El Servicio de Cardiología, un de los introductores de la Hemodinámica, cuenta con una 
importante unidad de Enfermedades coronarias. También destacan los avances en Neurocirugía y en Oftalmología.

Desde la dirección de Investigación y Docencia de la Vall d'Hebron se desarrollan diversos proyectos sobre enfermedades y tratamientos — cáncer, espina bífida, trasplantes pediátricos, inmunodeficiencias, hepatitis vírales, infecciones, trastornos del sueño, etc.-, o aspectos sanitarios diversos, como el proceso de atención de enfermería, la metodología diagnóstica o la transferencia de resultados entre laboratorios, entre otros. Independiente de ser hospitales de alta tecnología, los centros y sus profesionales tienen una vocación amplia, de atención a todos los niveles de patologías.

El interés por la prevención ha llevado al inicio de una línea de colaboración con la atención primaria de salud. La actividad asistencial del centro evolucionó notablemente con el aumento de la calidad de los hospitales comarcales, que hacen que un buen número de los pacientes que llegan en la Vall d'Hebron, los derivados de estos centros, acceda con diagnósticos preestablecidos.

En el año 1992, como en el resto de los hospitales del ICS, los de la Vall d'Hebron firman el primer contrato con el Servei Català de la Salut —SCS—, significando un paso decisivo hacia un modelo de gestión más empresarial y la mayor vinculación entre los ingresos y la facturación real por los servicios prestados.

- Área de influencia: Barcelonès.

- Tipología: Hospital de alta tecnología.

- Número de camas: 386.

- Servicios: Oncohematología. Pediatría. Especialidades médicoquirúrgicas pediátricas. Ginecología. Obstetricia. Unidad de Curas intensivas pediátricas. Neonatología y Nefrología.

\subsubsection{CIUTAT SANITÀRIA I UNIVERSITÀRIA DE BELLVITGE}

\subsubsection{ANTECEDENTES HISTÓRICOS}

El conjunto asistencial de la Ciutat Sanitària i Universitària de Bellvitge, además de otros edificios, la componen dos centros hospitalarios de máximo nivel, el Institut Català d'Oncologia y el Hospital Prínceps d'Espanya, inaugurado en el 8 de noviembre de 1972 por los, entonces príncipes, Juan Carlos de Borbón y Sofía de Grecia. 
El gran crecimiento demográfico de la década de los sesenta, motivado por el movimiento inmigratorio, y la insuficiencia de oferta sanitaria en la zona motiva la construcción.

El objetivo de la Ciutat Sanitària i Universitària de Bellvitge es proporcionar asistencia a su población, con calidad y con integración en el entorno social. Son objetivos de la ciutat sanitària, desenvolver de una manera continua procedimientos y técnicas que permitan garantizar la actualización permanente, y al mismo tiempo, reforzar su posición singular y diferenciada en el desarrollo de servicios especializados.

Entre los conceptos claves de la filosofía de la institución, están la implantación de la calidad integral y la definición de su oferta a partir de los criterios propios de una organización pública moderna, desde el ajuste presupuestario hasta el incremento de la eficiencia y de la competitividad de sus centros, comprometidos con el refuerzo constante de la función docente e investigadora.

\subsubsection{MISIÓN}

La finalidad de la Ciutat Sanitària i Universitària de Bellvitge es proporcionar servicios de asistencia sanitaria a su población, con calidad e integración en el entorno social. Ha de desarrollar de una manera continua procedimientos y técnicas que permitan garantizar su permanente actualización y reforzar su posición singular y diferenciada en la prestación y la evolución de servicios terciarios.

La calidad integral como concepto y la agilidad como objetivo prioritario serán elementos claves que marcarán las actuaciones de la Ciutat Sanitària, que adaptará su oferta de acuerdo con los objetivos que le atribuyen como organización pública.

El equilibrio presupuestario y el desarrollo de sistemas que permitan incrementar la eficiencia y aumentar la competitividad son acciones de las cuales dependerá el nivel de desarrollo de la Ciutat Sanitària i Universitària de Bellvitge, que buscará un permanente refuerzo de la función docente e investigadora. La creación de un valor diferencial y de pertenencia a una organización que quiere ser excelente será uno de los puntos que han de caracterizar las decisiones de actuación del personal y la elección del centro por parte de los pacientes. 


\subsubsection{ESTRUCTURA Y ORGANIZACIÓN}

La CSUB es una institución hospitalaria pública que pertenece al ICS y está acreditada como centro de alta tecnología — Grupo 4-. Está compuesta por diferentes edificios, el Hospital Prínceps d'Espanya; la antigua Escuela de Enfermería, compartido con la Universidad -Fundació Josep Finestres y Clínica Odontológica - ambos edificios están situados dentro del recinto urbanizado; el edificio del Hospital Duran i Reynals, compartido con el Institut Català d'Oncologia, Institut de Recerca Oncològica, el Institut de Diagnòstic per la Imatge y una base de SEMSA, este edificio está situado en el kilómetro 2,7 de la autovía de Castelldefels.

La mayor parte de los servicios de hospitalización están situados en el edificio del Hospital Prínceps d'Espanya, el bloque quirúrgico, las unidades de cuidados intensivos y enfermos críticos, las consultas externas, las urgencias y la mayor parte de los servicios centrales y de soporte. El resto de los servicios y unidades de apoyo, como también de las consultas externas, están distribuidos entre la antigua Escuela de Enfermería y el Hospital Duran i Reynals.

- Área de influencia: Regió Sanitària Costa de Ponent, Tarragona, Tortosa y Lleida.

- Tipología: Hospital de alta tecnología.

- Número de camas: 960.

- Servicios: Todas las especialidades.

- Áreas de investigación:

Área 1: Neurobiología.

Área 2: Cáncer.

Área 3: Patología infecciosa.

Área 4: Trasplante e inmunidad.

Área 5: Metabolismo, nutrición, crecimiento celular y arteriosclerosis.

Área 6: Genética humana.

Área 7: Enfermedades crónicas, degenerativas e inflamatorias. 
FIGURA 6.41. Organigrama de la CSU de Bellvitge.

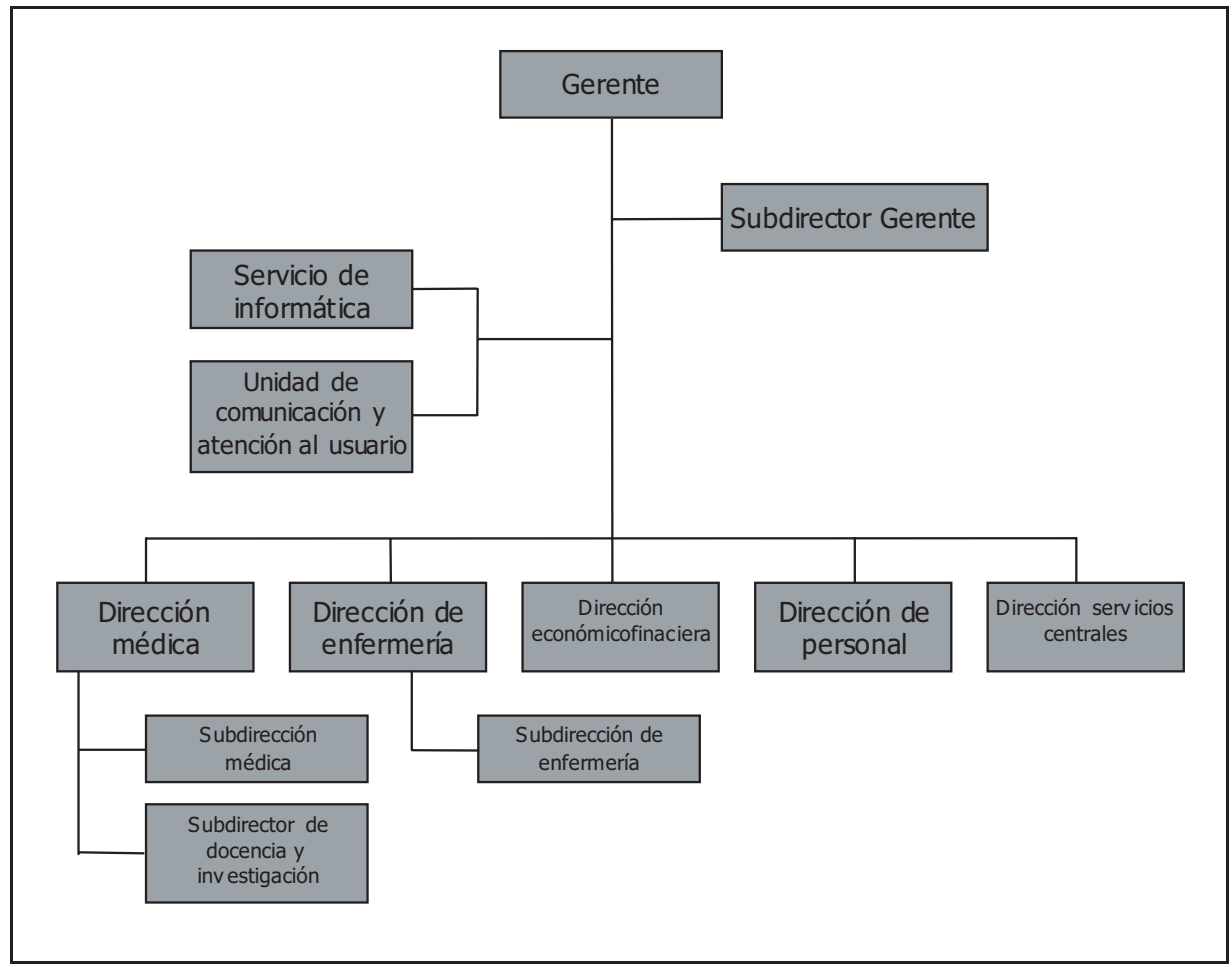

(C) Memòria 1997 Ciutat Sanitària i Universitària de Bellvitge [1998, p.23].

\subsubsection{HOSPITAL PRÍNCEPS D’ESPANYA}

\section{Antecedentes históricos}

El proyecto del Hospital Prínceps d'Espanya nace, por lo que consta, de una conversación. En el mes de enero de 1968, el ministro de Trabajo había asistido a la inauguración del ambulatorio de la Torrassa, en l'Hospitalet. El doctor Solanich, alcalde de la ciudad en aquellos momentos, le manifiesta la necesidad de disponer de una «residencia» para atender las necesidades de una población local que llegaba ya a las 160.000 personas. El ministro se muestra de acuerdo siempre y cuando tuviesen los terrenos.

Con relación al nombre del centro, en un principio se había de denominar Verge de Montserrat. En una visita de la princesa Sofía al Hospital Francisco Franco de Madrid, la actual Reina pregunta al doctor Bartolomé, que fue el primer director del Bellvitge, que nombre tendría el nuevo hospital. Aquel interés fue interpretado como una propuesta y de ahí surge el nombre actual. 
En el octubre de 1969 se compran por 78 millones de pesetas de la época las catorce hectáreas para la obra. La naturaleza de los terrenos complica y retarda los trabajos de fundamentación. Pero en dos años, un tiempo récord, en que se hacen turnos de día y noche, se completan los trabajos —en el mes de enero de 1970 se anuncia oficialmente la construcción y en el enero de 1972 la Seguridad Social acepta el edificio como hospital universitario-. El proyecto preveía una residencia de 25 plantas con 1.200 camas para 22 especialidades hospitalarias. Entretanto se hacen mejoras no previstas en el proyecto inicial, como la división de las unidades de Curas intensivas en «boxes» individuales o la ampliación de los espacios para la cocina y lavandería.

El hospital es resultado de los planteamientos emprendidos por el Instituto Nacional de Previsión - INP_, a partir de la Ley de Seguridad Social del año 1966. Bellvitge es un centro emblemático de la arquitectura de aquella época, de la cual forman también parte el Hospital de Traumatologia i Rehabilitació y el Materno-infantil de la Vall d'Hebron.

El edificio consta de tres bloques altos de veinte, trece y nueve plantas respectivamente, unidas por un núcleo común de comunicaciones verticales, de la cual derivan tres brazos para las Consultas externas, Urgencias y servicios de hostelería. El personal del centro tenía procedencias diversas, de hospitales barceloneses o de fuera, pero en buena parte procedían de la generación más joven de la Vall d'Hebron. En un principio, la organización de las especialidades se hace creando servicios médicos—quirúrgicos mixtos, dirigidos por los responsables de Cirugía, disciplina a la cual se dio preeminencia como símbolo de eficacia asistencial. Esta opción se fue modificando. En el verano de 1975, los profesionales del centro se suman a la reivindicación de una sanidad hospitalaria pública, que se impulsaba desde el movimiento progresista. Los médicos vinculados a estas opciones eran contrarios al mantenimiento, por parte de los facultativos de la sanidad pública, de una clientela privada propia. Los médicos internos y residentes — MIR — eran los impulsores de estas reivindicaciones. Bellvitge, muy sensibilizado por un entorno obrero comprometido en la transformación política, se transforma en un centro delantero de un proceso en que conviviera las reclamaciones laborales y la lucha contra la dictadura. La dirección tomó medidas represivas, con médicos expedientados y expulsados, que después con la democratización serían readmitidos.

Actualmente, el Hospital Prínceps de Espanya consolidó un proceso de calidad asistencial. Cuenta con 25 quirófanos y 35 camas para enfermos críticos. Entre las líneas de excelencia del centro destacan los trasplantes. 
Con relación a los trasplantes renales, se practican una media de 70 anuales, con una supervivencia del injerto superior al $90 \%$ en los cinco años, lo que sitúa el hospital entre los mejores de Europa. Este programa de trasplante renal empieza en el año 1980 y, desde entonces, se han hecho más de mil doscientos. En el mes de febrero de 1984 se realiza el primer trasplante hepático del Estado y, en estos momentos, se hacen una media superior a los treinta anuales. Y con referencia a los trasplantes de corazón, entre 1991 y 1993, se han hecho 35 intervenciones. Por otro lado, el servicio de Cirugía cardíaca ha practicado 7.770 intervenciones extracorpóreas entre 1974 y 1993 - 12.565 intervenciones de corazón y 15 casos de asistencia ventricular mecánica- La dotación tecnológica del hospital es otro de los factores de su calidad. La litotricia extracorpórea, la neurofisiología, la electrofisiología cardíaca o la angiografía digital, entre otros, son ejemplos de su alta tecnificación. También la informática, desarrollada en el propio centro, ha adquirido una gran importancia, con más de quince aplicaciones en funcionamiento, desde la estadística asistencial hasta la gestión de quirófanos. Con relación a los aspectos docentes, el campus de Bellvitge, con 3.000 estudiantes, está destinado a ser uno de los polos de estudios universitarios de Ciencias de la Salud de la Universitat de Barcelona, con enseñanzas de Medicina, Odontología, Podología, Enfermería y Salud Pública. La Ciutat Sanitària, también, está acreditada para impartir docencia de todas las especialidades para facultativos residentes desde 1972, al mismo tiempo que se han estado haciendo trabajos de investigación aplicada. Desde 1985, el hospital desarrolla investigación epidemiológica, clínica y experimental acreditada con el Fondo de Investigación Sanitaria de la Seguridad Social -FISS - y constituye la unidad de Investigación experimental, con tres áreas de actividad—quirófanos, laboratorio y estabularía—. En el año 1988, por otro lado, se crea la Fundación August Pi i Sunyer, para difundir y promover la investigación biomédica. La dinamización cultural del hospital, donde hasta existió una compañía de teatro estable —el Teatre Hospitalari Experimental-, se consolida notablemente con la puesta en funcionamiento, desde diciembre de 1989, de una galería de exposiciones que recibe el nombre de Bellvitge-Art, como servicio abierto a la ciudad. Cada año, también, se convoca un concurso de fotografías y de diseño infantil. El Hospital cuenta también con una biblioteca de préstamo de libros a los pacientes ingresados y familiares. 


\subsubsection{INSTITUT CATALÀ D'ONCOLOGIA}

\section{Antecedentes históricos}

El Institut Català d'Oncologia - ICO_ es una empresa pública creada por el Acuerdo del Gobierno de la Generalitat de Catalunya del 7 de febrero de 1995. El ICO es un centro de atención oncológica integral que nace como resultado de un proyecto de integración de las diferentes estrategias de lucha contra el cáncer en una sola institución, desde la investigación, epidemiología y la prevención hasta la oncología médica radioterápica, la hematología clínica o las curas paliativas. Sus orígenes se sitúan en la atención oncológica realizada en la Ciutat Sanitària i Universitària de Bellvitge y en la consolidación del servicio de oncología en muchos hospitales de Catalunya y, de los pioneros se destaca el Servei d'Oncologia de l'Hospital de la Santa Creu i Sant Pau, que fue creado en 1928.

Los objetivos básicos del ICO son los siguientes ${ }^{18}$ :

1. Ser el centro asistencial de referencia para el tratamiento de los pacientes diagnosticados con cáncer de la Región Sanitaria Costa de Ponent.

2. Actuar como centro de referencia para la realización de las técnicas terapéuticas de alta complejidad.

3. Asesorar el DSSS y el SCS en los ámbitos de la epidemiología, la prevención y el control del cáncer en Catalunya.

El Institut Català d'Oncologia está formado por dos áreas principales:

- La de epidemiología, prevención y control del cáncer.

- La de asistencial e investigación clínica.

Las áreas de investigación del ICO son las siguientes:

Área 1: Epidemiología y registro del cáncer.

Área 2: Física y protección radiológica.

Área 3: Hematología clínica.

Área 4: Oncología médica.

Área 5: Oncología radioterápica.

Área 6: Prevención y control del cáncer.

Con relación a la actividad asistencial, el ICO dispone de 126 camas de hospitalización, hospital de día, unidad de terapia intensiva para el trasplante de

18 Siguiendo parcialmente los objetivos básicos del ICO en la Memòria 1996 del Institut Català d'Oncologia [1997, p. 9]. 
células progenitoras hematopoéticas, aparatos de alta energía para el tratamiento con radioterapia y braquiterapia.

El ICO tiene como misión contribuir en la lucha contra el cáncer en Catalunya, por medio de la integración de las diferentes estrategias de tratamiento oncológico con la investigación epidemiológica, clínica, la prevención del cáncer y la docencia. Por tanto, los objetivos del ICO son en definitiva, investigar, prevenir, cuidar y mantener. Para conseguir estos objetivos, tiene personalidad jurídica propia por medio de su configuración como empresa pública. En 1996 se consolidó la oferta asistencial del Institut Català d'Oncologia, ya que la mitad de las unidades de hospitalización fueron abiertas durante el año 1995.

De forma global, el Institut Català d'Oncologia es visitado por unos 3.000 pacientes nuevos cada año, esto quiere decir, 1 de cada 8 pacientes diagnosticados de cáncer en Catalunya. Como corresponde a la tendencia actual de la atención oncológica, buena parte de esta atención se realiza en régimen ambulatorio, tanto en radioterapia como en hospital de día o consulta externa.

FIGURA 6.42. Organigrama del ICO.

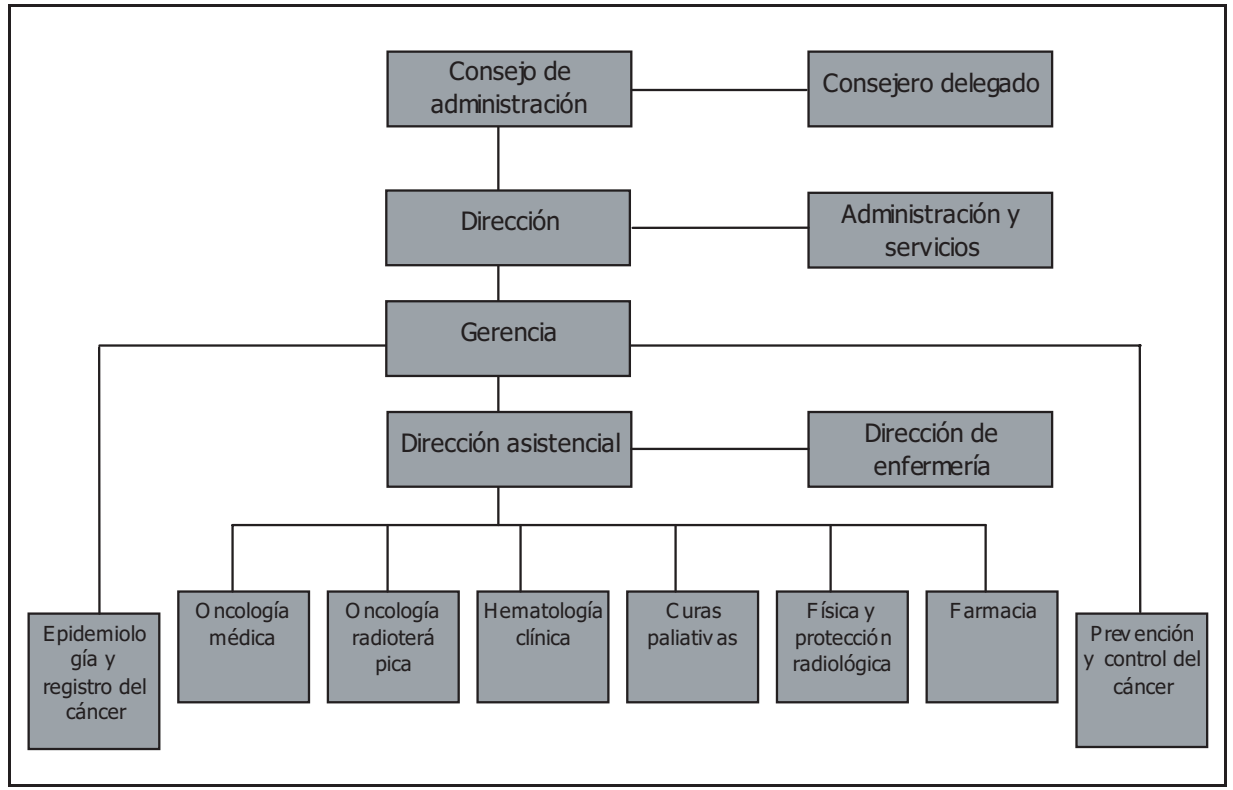

(C) Memòria 1997 del Institut Català d’Oncologia [1998, p. 127]. 


\subsubsection{HOSPITAL UNIVERSITARI GERMANS TRIAS I PUJOL}

\subsubsection{ANTECEDENTES HISTÓRICOS}

Construido entre 1972 y 1979, fue concebido para acoger 1.200 camas, contaba con una estructura mixta, horizontal y vertical, que definía dos entes entendidos como los dos hospitales, el general y el materno-infantil, de una «ciudad sanitaria» arquitectónicamente compacta. Es el actual Hospital de Badalona Germans Trias i Pujol.

Hijos de la ciudad, los hermanos Trias y Pujol, Joaquim [1887-1964] y Antoni [1891-1970], fueron dos destacados cirujanos y catedráticos, responsables de la organización y dirección de los estudios de Medicina de la primera Universitat Autònoma de Barcelona, y de la Escuela de Enfermería de la Generalitat Republicana.

Para muchos badaloneses los hermanos Trias y Pujol son conocidos por el nombre de la línea que separa la parte alta de los valles de Canyet y de Pomar: Can Ruti — este nombre proviene de la antigua masía que fue propiedad da la familia Urrútia, desde principios del siglo XVIII. La leyenda habla que Joan Urrútia, en 1714, terminado el sitio de la ciudad sale de Barcelona y deja galopar su caballo hasta que se para delante de una masada, que compró y donde se afincó-. Este emplazamiento, lejos del núcleo urbano, fue uno de los factores condicionantes de la realidad del centro.

Oficialmente, el centro abrió sus puertas el 14 de abril de 1983, con 166 camas repartidas mayoritariamente entre tres grandes servicios: Medicina interna, Cirugía general y digestiva, y Traumatología y Cirugía ortopédica. Como unidades menores se dotan también camas para Neumología, Oncología médica, Hematología y Medicina intensiva. El año siguiente se pone en servicio la unidad de Urgencias. La situación económica del sector hospitalario en aquellos años dificulta el desarrollo integral y armónico de su estructura asistencial y administrativa, y este hecho fue la causa de un cierto descontento entre los usuarios iniciales. Independientemente de esto, cuatro años después de la apertura del hospital funcionaban ya una treintena de servicios. En enero de 1987 se pone en marcha los servicios de Cardiología, Neurología y Neurocirugía, y en 23 de noviembre de 1987 se inauguraba la unidad pediátrica del hospital con los servicios de Pediatría, Cirugía pediátrica y la unidad neonatal. Dos meses antes se habían trasladado al centro los recursos del Hospital Maternal de Santa Coloma de Gramenet. 
Con eso, se llegó a las 401 camas, y en los años siguientes el crecimiento de la hospitalización continuó hasta llegar a las 545 camas, el día 1 de octubre de 1990.

Entre las ofertas asistenciales del centro se destacan los hospitales de día para los tratamientos oncológicos y delSIDA, y las unidades de desintoxicación, de trastornos de la alimentación y las de trasplantes renales — desde 1985-, de páncreas y de córneas. La incorporación de los aparatos de alta tecnología fue una de las líneas de avance del centro. Además del aparato de tomografía axial computarizada - TAC—, durante el 1993 se ponen en marcha el acelerador lineal y un simulador para el servicio de Radioterapia, y una gammacámara — con habitaciones aisladas- para Medicina nuclear.

El hospital se consolidó como centro docente, tanto en formación de pregrado como de postgrado. En 1985 se incorporan, con poco tiempo de diferencia, la primera promoción de médicos internos residentes - MIR - y estudiantes de tercer curso de la facultad de Medicina de la Universitat Autònoma - UAB —. El año siguiente se acreditó definitivamente el programa MIR, que imparte 29 especialidades. El 19 de marzo de 1990 el centro se consolida como hospital universitario con la firma del convenio entre la UAB y el Institut Català de la Salut. También es fluida la colaboración del centro con las escuelas de Enfermería y de Fisioterapia de Santa Coloma de Gramenet.

La unidad de Investigación del hospital, creada en 1988, fue acreditada por el Departament de Sanitat i Seguretat Social en 1991 y reconocida por el Ministerio de Sanidad y Consumo en 1992. Se estructura en dos áreas: el Centro de Experimentación Animal y el Centro de Investigaciones Sanitarias, que cuentan con una estructura común de los servicios asistenciales, la epidemiología clínica y la biblioteca central.

Algunas de las líneas de excelencia de la investigación del centro son los trabajos sobre autoinmunidad, fisiopatología respiratoria, neoplasias, nutrición clínica o enfermedades infecciosas. En diciembre de 1993, en el ámbito del servicio de Microbiología se abrió el primer laboratorio de retrovirología de Catalunya —el segundo de España—, en que se investiga sobre el virus del SIDA. Esta nueva dotación fue fruto de un convenio de colaboración entre la Fundación Privada de Lucha contra el SIDA, la Fundación «la Caixa» y el Departament de Sanitat i Seguretat Social. 


\subsubsection{ESTRUCTURA Y ORGANIZACIÓN}

El HUGTiP es un hospital del ICS acreditado como centro de alta tecnología - Grupo 4-. Tiene también la acreditación de la Unitat d'Investigació por el programa de la Red Estatal de Unidades de Investigación — REUNI—.

FIGURA 6.43. Organigrama del HUGTiP.

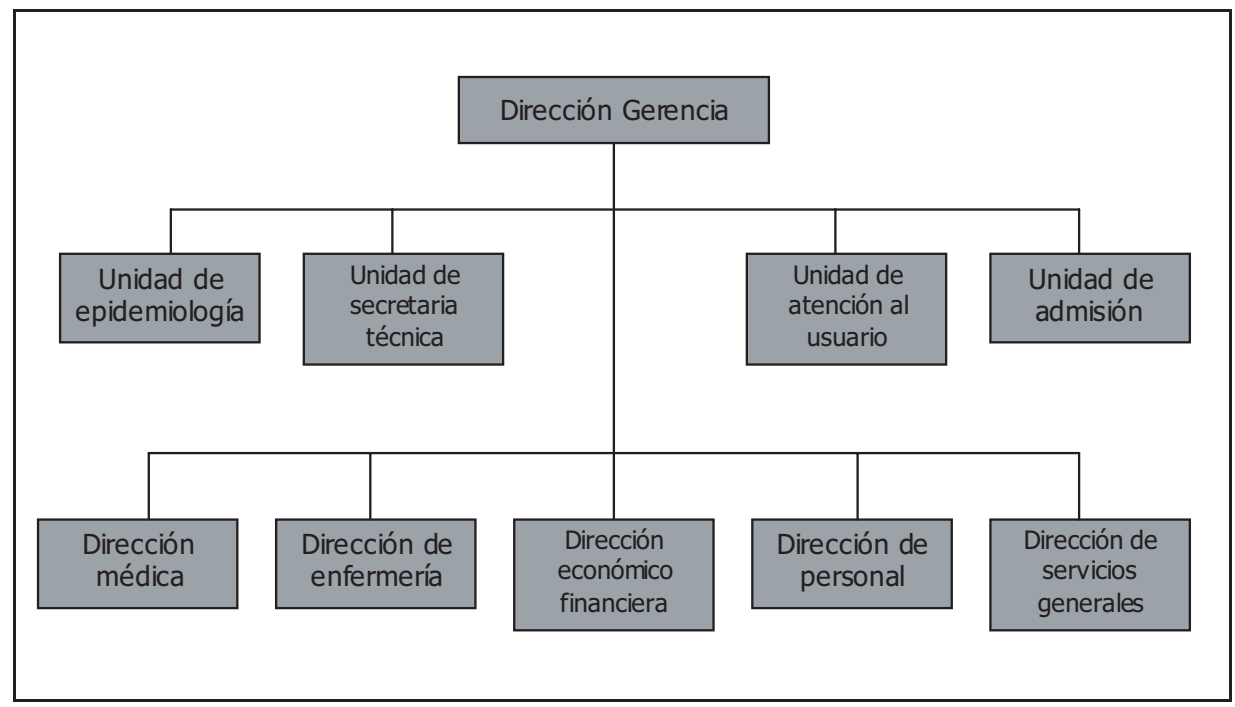

(C) Memòria general 1996 HUGTiP [1998, p. 13].

El hospital está inmerso en un cambio cultural propiciando la introducción de la dirección por objetivos como instrumento de trabajo participativo.

- Área de influencia: Barcelonès y Maresme.

- Tipología: Hospital de alta tecnología.

- Número de camas: 573.

- Servicios: Todas las especialidades, excepto la cirugía cardíaca.

- Áreas de investigación:

Área 1: Tolerancia y autoinmunidad.

Área 2: Nutrición artificial y fisiopatología digestiva.

Área 3: Biología molecular del cáncer.

Área 4: Microbacterias e infección respiratoria.

Área 5: SIDA y VIH.

Área 6: Fisiopatología cardiorrespiratoria.

Área 7: Epidemiología clínica y molecular. 
Área 8: Leucemia y diferenciación hematopoéticas.

Área 9: Enfermedades tromboembólicas.

Área 10: Modelos experimentales de la enfermedad animal.

Área 11: Fisiopatología de la enfermedad inflamatoria idiomática.

Área 12: Trastornos de la alimentación.

Área 13: Fisiopatología del dolor.

Área 14: Fisiopatología de la insuficiencia renal y de la hipertensión.

Área 15: Metodología diagnóstica y práctica clínica.

Área 16: Nuevos procedimientos en cirugía ortopédica.

Área 17: Enfermedades infecciosas y microbiología clínica.

Área 18: Patología intraestructural.

Área 19: Epidemiología de la infección por VIH—SIDA.

Área 20: Inmunología de la piel. 